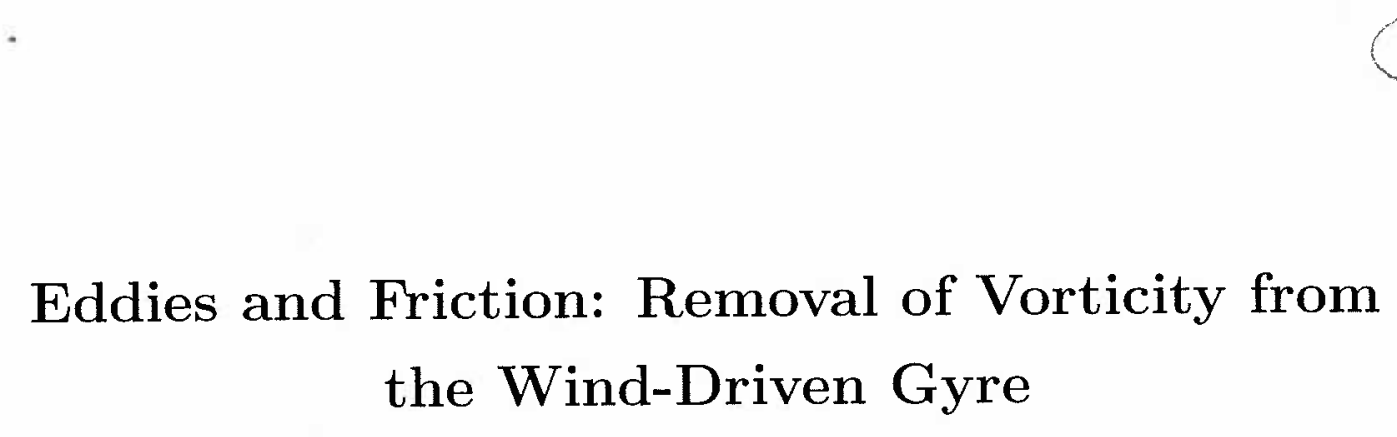

by

\author{
Baylor Fox-Kemper
}

B.A., Reed College, 1996; M.A., Brandeis University, 1998

Submitted in partial fulfillment of the requirements for the degree of

Doctor of Philosophy

at the

MASSACHUSETTS INSTITUTE OF TECHNOLOGY

and the

MBL / WHOI

LIBRARY

Woods Hole, MA

Morhe Biological Laboratory

Woods Hole Oceanographts

WOODS HOLE OCEANOGRAPHIC INSTITUTION

June 2003

(C) Baylor Fox-Kemper, MMIII. All rights reserved,

The author hereby grants to MIT and WHOI permission to reproduce and distribute publicly paper and electronic copies of this thesis document in whole or in part.

Author

Joint Program in Oceanegraphy Applied Ocean Science and Engineering

March 8, 2003

Certified by

Joseph Pedlosky

Senior Scientist

Thesis Supervisor

Certified by

Paola Malanotte-Rizzoli

Professor

Thesis Supervisor

Accepted by

Carl Wunsch

Chair, Joint Committee for Physical Oceanography 


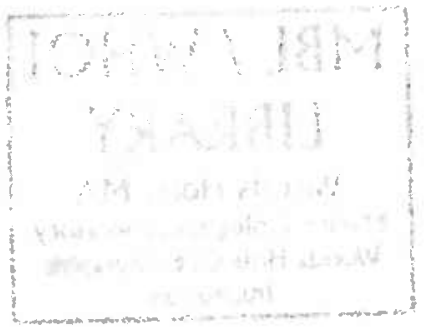




\title{
Eddies and Friction: Removal of Vorticity from the Wind-Driven Gyre
}

\author{
by \\ Baylor Fox-Kemper
}

\author{
Submitted to the Joint Program in Oceanography/Applied Ocean Science and \\ Engineering \\ on March 11, 2003, in partial fulfillment of the \\ requirements for the degree of \\ Doctor of Philosophy
}

\begin{abstract}
Inertial terms dominate the single-gyre ocean model and prevent western-intensification when the viscosity is small. This occurs long before the oceanically-appropriate parameter range. It is demonstrated here that the circulation is controlled if a mechanism for ultimate removal of vorticity exists, even if it is active only in a narrow region near the boundary.

Vorticity removal is modeled here as a viscosity enhanced very near the solid boundaries to roughly parameterize missing boundary physics like topographic interaction and three dimensional turbulence over the shelf. This boundary-enhanced viscosity allows western-intensified mean flows even when the inertial boundary width. is much wider than the frictional region because eddies flux vorticity from within the interior streamlines to the frictional region for removal.

Using boundary-enhanced viscosity, western-intensified calculations are possible with lower interior viscosity than in previous studies. Interesting behaviors result: a boundary-layer balance novel to the model, calculations with promise for eddy parameterization, eddy-driven gyres rotating opposite the wind, and temporal complexity including basin resonances.

I also demonstrate that multiple-gyre calculations have weaker mean circulation than single-gyres with the same viscosity and subtropical forcing. Despite traditional understanding, almost no inter-gyre flux occurs if no-slip boundary conditions are used. The inter-gyre eddy flux is in control only with exactly symmetric gyres and free slip boundaries.

Even without the inter-gyre flux, the multiple-gyre circulation is weak because of sinuous instabilities on the jet which are not present in the single-gyre model. These modes efficiently flux vorticity to the boundary and reduce the circulation without an inter-gyre flux, postponing inertial domination to much smaller viscosities.
\end{abstract}


Then sinuous modes in combination with boundary-enhanced viscosity can control the circulation.

Thesis Supervisor: Joseph Pedlosky

Title: Senior Scientist

Thesis Supervisor: Paola Malanotte-Rizzoli

Title: Professor 


\section{Acknowledgments}

Around the axis we all spin,

To determine what's left out and what's left in.

-Peter Gabriel, No Way Out

For Miles, whose imminent (and eminent) arrival got me working overtime. I look forward to the day when I can tell you what this dissertation shows.

The biggest thanks go to Jordan. While I was churning away in the office, you kept our real life going and wonderful. I will probably never be able to make it up to you, but I will try.

Thanks to my advisors, Joseph Pedlosky and Paola Rizzoli. They have stood by me with only occasional doubts. Even when the model wasn't working and the way forward seemed dim, their encouragement was steadfast. I thank Paola, who always trusted my judgment. I thank Joe, who checked every symbol and every punctuation mark in everything I gave him, even when it didn't deserve it. Also, Joe deserves special thanks for suggesting the idea of using variable viscosity.

Thanks to my other committee members, Glenn Flierl, Vitalii Sheremet, and Mike Spall for their suggestions and useful criticism. I also must thank them for always keeping the discourse constructive, even when they didn't agree with me. Thanks also to Raf Ferrari for giving a warm introduction at my defense, serving as chair, and also for being someone who was always willing to talk.

Thanks also to my unofficial and temporary committee members, who through their writings and suggestions I benefited enormously: Steve Meacham, Glenn Ierley, John Marshall, Carl Wunsch, John Boyd, and Steven Orszag. Special thanks are due to Steve Jayne who contributed almost all of the computer resources.

Thanks to all the students who helped, both emotionally and technically. My officemate Kerim was always someone I could call when I had trouble. I am greatly indebted to him. Markus has been a good friend and role model to me for getting 
through the Joint Program unscathed. Good luck to my classmates in oceanography and out.

Over the course of my graduate career, I have received funding from a number of sources. Most recently, I have been supported by a research assistantship through Joe Pedlosky. Before that, I received the MIT Presidential Fellowship, and before that I received the DOD/NDSEG Graduate Fellowship sponsored by the Office of Naval Research. While obtaining my master's I received a fellowship from the Gillette Corporation. I thank all of these organizations for their help. 


\section{Contents}

1 Introduction $\quad 11$

1.1 Westward Intensification . . . . . . . . . . . . . . . 11

1.1.1 Previous Studies Using the Homogeneous Model . . . . . . 12

1.1.2 What Sets the Wind-Driven Circulation Strength? . . . . . 33

1.1 .3 The Role of Vorticity . . . . . . . . . . . . . . 35

1.2 Dissertation Goals and Choice of Model . . . . . . . . . . . . 38

1.2.1 Definition of Eddy and Mean . . . . . . . . . . . . . . . 42

1.2.2 Summary of the Chapters ... . . . . . . . . . . 43

2 Control of the Wind-Driven Single Gyre by Eddy Fluxes of Vorticity to a Region of Enhanced Viscosity 45

2.1 Boundary-Enhanced Viscosity in the Homogeneous Model . . . . . . 49

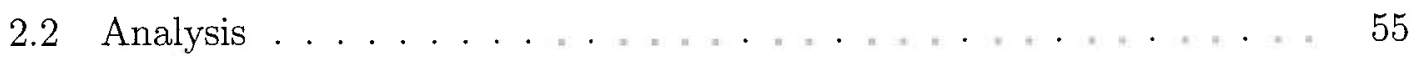

2.2.1 Interpreting Eddy Flux Divergences . . . . . . . . . . . 59

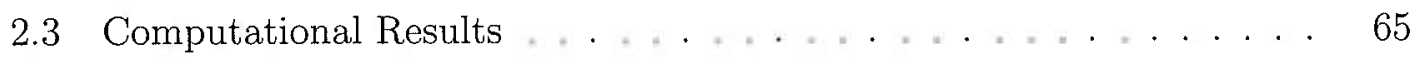

2.3.1 Control of Circulation Strength ... . . . . . . . . 65

2.3.2 Mechanism of Vorticity Transport . . . . . . . . . . . 70

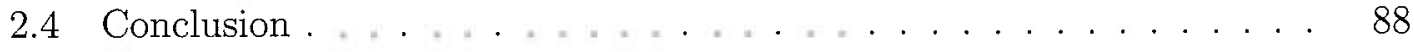

3 Eddies' Effects in the Single-Gyre Model with Low Interior Viscosity 93

3.1 Boundary Layer Balances . . . . . . . . . . . . . . . . . 94 
3.2 Homoparic Calculations $\ldots \ldots \ldots \ldots$

3.3 Counter-Rotating Gyres . . . . . . . . . . . . . . . . . 104

3.4 Temporal Structure of the Solutions . . . . . . . . . . . . . 113

3.5 Presence of Basin Modes . . . . . . . . . . . . . . . 125

3.6 Energetics . . . . . . . . . . . . . . . . . . . . . . . 138

3.7 Bottom Friction . . . . . . . . . . . . . . . . . . . . . 145

3.8 Slip Boundary Conditions . . . . . . . . . . . . . . . . 153

3.9 Conclusion . . . . . . . . . . . . . . . . . . . 158

4 Adding a Second Gyre 163

4.1 The Single-gyre, Double-gyre and Two-gyre Models . . . . . . . 167

4.2 Results with No-Slip Boundary Conditions . . . . . . . . . . . . . 169

4.2.1 Frictional and Eddy Fluxes . . . . . . . . . . . . . 171

4.3 Results with Slip Boundary Conditions . . . . . . . . . . . . 182

4.4 Mechanisms for Control of Circulation Strength . . . . . . . 200

4.4.1 Inter-gyre Eddy Transport . . . . . . . . . . . . . . 201

4.4 .2 Dissipative Meandering . . . . . . . . . . . . . . . 206

4.4.3 Exposure to Opposing Wind Forcing . . . . . . . . . . 213

4.4.4 Sinuous Modes: Breaking the Initial Symmetry of the DoubleGyre . . . . . . . . . . . . . . . . . . . 216

4.5 Boundary-Enhanced Viscosity with a Second Gyre . . . . . . . 234

4.6 Conclusion . . . . . . . . . . . . . . . . . . . . . . . . 240

5 Conclusion 243

5.1 Wind-Driven Circulation Control by Boundary-Enhanced Viscosity . 243

5.2 Method of Westward Intensification at High Reynolds Number . . . . 250

5.3 Behaviors of the Relatively Inviscid Single-Gyre Model _. . . . . 251

5.3.1 Higher Reynolds Number? . . . . . . . . . . . . . . . 253

5.4 Future Directions of Research _. . . . . . . . . . . 254 
5.4.1 Toward a Realistic Model of Vorticity Removal at the Boundary 254

5.4 .2 Topographic Interaction . . . . . . . . . . . . . . . 255

5.4 .3 Density Layer Outcropping . . . . . . . . . . . . . 257

5.4 .4 Other Processes . . . . . . . . . . . . . . . . . . 259

5.4 .5 Sinuous Modes . . . . . . . . . . . . . . . 260

5.4.6 The Three-Dimensional Problem . . . . . . . . . . . . . . 261

5.4 .7 Eddy Parameterization . . . . . . . . . . . . 262

5.5 Final Remarks . . . . . . . . . . . . . . . . . . 264

A Properties of the Chebyshev Polynomials $\quad 267$

A.1 Definitions and Basic Identities _. . . . . . . . . . . 267

A.2 Chebyshev Polynomial Expansion to Approximate Functions . . . . 269

A.3 2-dimensional Expansions . . . . . . . . . . . . . . 271

A.4 Boundary Conditions . . . . . . . . . . . . . . . . . . 272

B The Steady-State Numerical Models $\quad 275$

B.1 Newton's Method . . . . . . . . . . . . . . . . . 275

B.1.1 One-Dimensional Newton's Method . . . . . . . . . . 275

B.1.2 Multi-dimensional Newton's Method . . . . . . . . . . 276

B.1.3 Evaluation of the Jacobian . . . . . . . . . . . 278

B.2 Finite Difference Model . . . . . . . . . . . . . . . . . . . . 278

B.3 Chebyshev Pseudo-Spectral Model . . . . . . . . . . . . . 279

C The Time-Dependent Numerical Model 283

C.1 Implementing The Field Equations . . . . . . . . . . . . . . . . 283

C.2 Inversion of Poisson and Helmholtz Equations . . . . . . . . . 285

C.3 Setting the Boundary Conditions . . . . . . . . . . . . . . . 287

C.4 Spinning up the Model . . . . . . . . . . . . . . . . . 288

C.5 Sources of Numerical Error . . . . . . . . . . . . . . . . . . . 288 


\section{Chapter 1}

\section{Introduction}

\subsection{Westward Intensification}

Perhaps the most striking feature of the general oceanic wind-driven circulation is the intense crowding of streamlines near the western borders of the oceans. The Gulf Stream, the Kuroshio, and the Agulhas Current are examples of this phenomenon. The physical reason for the westward crowding of streamlines has always been obscure. The purpose of this paper is to study the dynamics of wind-driven oceanic circulation using analytically simple systems in an attempt to discover a physical parameter capable of producing the crowding of streamlines.

-Stommel (1948)

There is a peculiar physical mechanism at work in the formation of the western boundary currents. The fluid chooses a location for the boundary current by compass direction, a result which is relatively independent of forcing, details of the local topography, and the sense of rotation of the earth (as there are still western boundary currents in the Southern Hemisphere).

The purpose of this dissertation is to explore the mechanisms which allow Stommel's 'physical parameter capable of producing the crowding of streamlines' to con- 
tinue to function as the models of the ocean become more inviscid and time-dependent.

The thesis of this dissertation is that the effects of boundaries and the effects of eddies work together as an important control on the strength of the circulation even as the ocean becomes less viscous. In relatively inviscid models of the ocean, the input of vorticity from the wind's forcing is transported by eddies toward the boundary where it is removed by processes that can only be found at the edges of the ocean.

\subsubsection{Previous Studies Using the Homogeneous Model}

Before I introduce the topics covered in this dissertation in detail and summarize the chapters, it is helpful to place the questions asked here in context with previous work.

A model which is equivalent to the one Stommel used to propose his theory of westward intensification has come to be known as the homogeneous ocean model. In this model, the fluid is considered to be rapidly rotating and of uniform density. Traditionally, it is also assumed that there is a constant depth of fluid. These assumptions allow the Navier-Stokes equations of fluid motion to be reduced to a single equation for the streamfunction of the 2-dimensional motion $\left(\frac{\partial \psi}{\partial y}=-u, \frac{\partial \psi}{\partial x}=v\right)$. In the nondimensional form used in this dissertation, it is

$$
\frac{\partial \nabla^{2} \psi}{\partial t}+\nabla \cdot\left(\hat{\mathbf{x}} \psi-\hat{\mathbf{x}} \delta_{I}^{2} \frac{\partial \psi}{\partial y} \nabla^{2} \psi+\hat{\mathbf{y}} \delta_{I}^{2} \frac{\partial \psi}{\partial x} \nabla^{2} \psi-\delta_{M}^{3} \nabla \nabla^{2} \psi+\delta_{S} \nabla \psi\right)=w_{E} \cdot(1.1)
$$

Stommel solved for a square ocean, and during nondimensionalization the square becomes unit length in each direction. The variable $\psi$ is the streamfunction of the

depth-integrated velocity $\left(u=-\frac{\partial \psi}{\partial y}\right.$ and $\left.v=\frac{\partial \psi}{\partial x}\right)$. This equation is called the vorticity equation, because it describes the time evolution of $\nabla^{2} \psi$, the component of relative vorticity in the vertical direction.

The nondimensionalization has used the following definitions (asterisks denote 
dimensional quantities):

$$
\begin{aligned}
w_{E *} \equiv W_{E} w_{E} & \delta_{I}^{2} \equiv \frac{W_{E} f_{0}}{\beta^{2} D L^{2}} \\
\delta_{S} \equiv \frac{r}{\beta L} & \delta_{M}^{3} \equiv \frac{A_{H}}{\beta L^{3}} \\
\psi_{*} \equiv \frac{W_{E} f_{0} L}{\beta D} \psi & t_{*} \equiv \frac{t}{\beta L} \\
x_{*} \equiv L x & y_{*} \equiv L y
\end{aligned}
$$

The maximum of the wind's vorticity input is $W_{E}$. Twice the rate of angular rotation of the frame is $f$. Its value in the center of the domain is $f_{0}$, and its meridional derivative is $\beta$. The depth of the moving fluid is $D$, and the zonal width of the basin is $L$. The horizontal eddy viscosity, or Austausch coefficient, is $A_{H}$. The decay rate due to the bottom drag is $r$.

This nondimensional form is chosen so that the coefficients are powers of the widths of the boundary layers in various traditional models.

All of the models proposed here will be considered to be driven by the wind (although other forcing mechanisms can be expressed similarly as sources of vorticity). Thus, the function $w_{E}$ is the vorticity supplied by the forcing of the wind.

\section{Linear Solutions: Breaking the Symmetries}

The first truly useful result for studying the wind-driven ocean circulation was found by Sverdrup (1947). He combined the depth-integrated, linearized horizontal momentum equations with a wind-stress and the continuity equation. This gave him cause to take the derivative of the Coriolis parameter and thereby derive an equation which distinguishes the north-south direction.

Written in the nomenclature of the vorticity equation 1.1, Sverdrup's solution is

$$
\psi_{I}=\int_{x_{e}}^{x} w_{E} d x^{\prime}
$$


Only one boundary condition is used to solve for this solution: the requirement that the streamfunction vanish at the eastern boundary $\left(\psi\left(x=x_{e}\right)=0\right){ }^{1}$ This automatically causes the eastern boundary to be impermeable to the fluid.

Sverdrup's solution will be referred to frequently in this dissertation as it applies wherever friction and nonlinearity are negligible. In fact, the nondimensionalization of equation 1.1 is constructed to utilize Sverdrup's solution in the scaling of the streamfunction. The wind forcing will not vary in the zonal direction, and the maximum magnitude of the wind forcing will always be 1 , and the basin width is scaled to be 1 . Thus, equation 1.6 can be integrated for Sverdrup's solution in this nondimensional form, and the maximum magnitude of the streamfunction will be 1 (at $x=0$ ).

Sverdrup realized that since this result uses only the depth-integrated equations of motion, this balance might hold for baroclinic flows as well as barotropic. In fact, the Sverdrup relation is one of the only simple constraints on a baroclinic flow. Many subsequent theories (e.g., the thermocline theory of Luyten et al. (1983)) use the Sverdrup balance as a starting point precisely because of its simplicity. This dissertation will frequently return to whether the Sverdrup balance holds in a model calculation. This is an important issue in assessing the generality of theories such as that of Luyten et al. (1983).

Sverdrup's theory breaks the symmetry of 2-dimensional space by distinguishing the northern direction with the use of the gradient of the Coriolis parameter. The resulting anisotropic term in the vorticity equation, $\nabla \cdot(\hat{\mathbf{x}} \psi)=\frac{\partial \psi}{\partial x}$, is usually called the $\beta$-term, because $\beta$ is the typical notation for the derivative of the Coriolis parameter, although it does not appear in the equations above because they are nondimensionalized using it as a unit measure. It results from the advection by the fluid of the fluid's vorticity due to the rotation of the Earth. To demonstrate its anisotropy, consider a 90 degree counter-clockwise rotation of the coordinate axes: replace $x$ with $y$ and $y$

\footnotetext{
${ }^{1}$ In this dissertation, the impermeability of the basin will be implemented by setting $\psi$ to zero on the boundary. This is not necessary, or even correct in some models. However, in the context of the homogeneous model, it is appropriate.
} 
with $-x$ in the vorticity equation 1.1. Only this term changes in sign. Thus, were it not for this term, no direction would be uniquely selected as any solution of the equation would remain a solution were it rotated by 90 degrees.

Stommel (1948) was the first to group together the dynamics of the western boundary currents with the anisotropy of the $\beta$-term. He used a simple model of an ocean basin: a rectangular impermeable basin with wind, the $\beta$-term, and bottom friction, $-\delta_{S} \nabla^{2} \psi$. This resulted in the vorticity equation 1.7 .

$$
\frac{\partial \psi}{\partial x}=w_{E}-\delta_{S} \nabla^{2} \psi
$$

Stommel chose a particular wind stress $\left(\tau_{x}=-\sin (\pi y / L)\right)$ and solved this equation, but this equation would have applied to any wind stress. The solution is given in equation 1.8 for a case where $w_{E}$ varies only in $y$ and $\delta_{S} \ll 1$. I have included a plot of the Stommel (1948) solution (figure 1-1a) for the same wind forcing Stommel used: $w_{E}=-\sin \pi y$.

$$
\psi=\psi_{I}\left(1-e^{-x / \delta_{S}}\right)
$$

The asymptotic solution in the interior $\left(x \gg \delta_{S}\right)$ of Stommel's model is just $\psi_{I}$ from Sverdrup's solution (equation 1.6). Near the western boundary Stommel's solution diverges from Sverdrup's in order to satisfy the impermeable western boundary condition. A second boundary condition can be imposed because the bottom friction term raises the differential order of the vorticity equation. Because the bottom friction term is the only second-order term, it must be responsible for satisfying the second boundary condition, and it is therefore not surprising to find it to be a large contributor in the region of the western boundary where the second boundary condition must be satisfied.

Stommel pointed out that without the gradient in Coriolis parameter the model is symmetric in the east-west direction. He demonstrated this with a plot of the solution without the $\beta$-term. Here it suffices to note that Stommel's vorticity equation without 
the $\beta$-term is Poisson's equation and is therefore obviously isotropic.

Equation 1.1 is anisotropic because of the $\beta$-term. Sverdrup retained just enough terms to distinguish north, while Stommel's model (equation 1.7) has a second asymmetry - it knows the difference between east and west intrinsically. Sverdrup's model, on the other hand, had a vorticity equation which did not distinguish east from west; Sverdrup's model distinguishes east and west only by the choice of where the impermeability boundary condition is satisfied. Consider a switching $x$ to $-x$ in the equation for Stommel's model (equation 1.7), since the $\beta$-term changes sign while the bottom friction doesn't, clearly the equation changes. Because the $\beta$-term has only one $x$-derivative while the friction term has two, the solution must exponentially decay from west to east. Thus, it is not possible in Stommel's model to substitute an eastern boundary current, the boundary current must be in the west.

Munk's first paper on westward intensification came soon after Stommel's (Munk (1950)) Munk and Carrier (1950) followed up with more realistic basin shapes and observed wind stresses. Munk's achievement was to use a lateral friction with constant viscosity $\left(\delta_{M}^{3} \nabla^{4} \psi\right)$ instead of the bottom drag used by Stommel. The advantage of this form of friction is that its viscosity can be thought of as eddy viscosity, a topic discussed further below. He solved the vorticity equation given by equation 1.9.

$$
\frac{\partial \psi}{\partial x}=\hat{k} \cdot \nabla \times \vec{\tau}+\delta_{M}^{3} \nabla^{4} \psi
$$

This vorticity equation requires an additional boundary condition at each boundary. Munk used no-slip, where the tangential velocity vanishes at the boundaries $\left(\frac{\partial \psi}{\partial n}=0\right)$. This is the boundary condition appropriate for the solution of the Navier-Stokes equations from which the vorticity equation is derived. However, the viscosity in Munk's model is not intended to represent molecular viscosity, it is intended to be an eddy viscosity, a representation of the mixing by eddies. Because it is not clear that the parameterization of eddies should obey the same boundary conditions as that for molecular viscosity, Welander (1964) provided the solution for slip boundary 
conditions (also known as no-stress or free-slip). These boundary conditions require that the normal stress vanishes at the boundaries $\left(\nabla^{2} \psi=0\right)$. Both of these boundary conditions are used in this dissertation, and they produce quite different results.

In the case of no-slip boundary conditions, Munk's solution is

$$
\psi=\psi_{I}\left[1-e^{-x / 2 \delta_{M}}\left(\cos \frac{\sqrt{3} x}{2 \delta_{M}}+\frac{1}{\sqrt{3}} \sin \frac{\sqrt{3} x}{2 \delta_{M}}\right)\right]
$$

In the case of slip boundary conditions, Welander's solution to the Munk problem is

$$
\psi=\psi_{I}\left[1-e^{-x / 2 \delta_{M}}\left(\cos \frac{\sqrt{3} x}{2 \delta_{M}}-\frac{1}{\sqrt{3}} \sin \frac{\sqrt{3} x}{2 \delta_{M}}\right)\right]
$$

I have included a plot of the Munk (1950) solution with no-slip boundary conditions (figure 1-2a).

As in Stommel's solution, Munk's solution asymptotically approaches the Sverdrup balance as $x$ becomes much larger than $\delta_{M}$, and like Stommel's solution the east-west symmetry-breaking occurs because the $\beta$-term changes sign upon changing $x$ to $-x$ while the frictional term does not. Also as in Stommel's model, the frictional term is the term which raises the differential order requiring the imposition of additional boundary conditions beyond no-normal flow at the eastern boundary. In fact, because the differential order is raised to four, three additional boundary conditions are required: no-normal flow in the west, and either no-slip or no-stress at both the eastern and western boundaries. In the same way as in Stommel's model, the western boundary is the only choice for the boundary layer which avoids exponential growth in the streamfunction as one moves away from the boundary.

The wind-driven solutions of Munk (1950), Stommel (1948), and Sverdrup (1947) all share the same interior flow in which the advection of planetary vorticity balances the vorticity input of the wind. In Munk's model and Stommel's model, which are closed, the vorticity input is subsequently removed by a frictional term.

The vorticity equation 1.1 is an equation which can be used to budget the import 
and export of vorticity from region to region. When inertia is included but there are no frictional terms, the absolute vorticity $\delta_{I}^{2} \nabla^{2} \psi+y$ is conserved following the motion of a fluid parcel. On the other hand, when inertial terms are absent, the advection of relative vorticity is neglected; only the planetary vorticity is advected.

Because Stommel's and Munk's models are linear, relative vorticity can not be advected. This requires that any change in the latitude of the fluid be accompanied by a change in vorticity to match the new local planetary vorticity. In the Sverdrup balance, this change is accomplished by the wind forcing, but in the frictional boundary layer, this change must be accomplished by friction. It is not difficult to show that this implies that the vorticity input by the wind at a particular latitude must be removed by the friction at that same latitude.

Pedlosky (1965a) presented another way to break the symmetry between west and east: the propagation of Rossby waves. Free Rossby waves in this model are solutions to the equation

$$
\frac{\partial \nabla^{2}}{\partial t} \psi+\nabla \cdot(\hat{\mathbf{x}} \psi)=0
$$

Again, the $\beta$-term changes sign when the sign of $x$ changes but another term, the rate of the vorticity change with time, does not. Thus, Rossby waves are not symmetric in behavior to the east and west. When put in physical terms, Rossby waves have different wavelengths when their group velocity is eastward rather than westward. Westward propagating waves are longer than eastward propagating ones. Eastward propagating waves obey $k^{2}>l^{2}$ while their westward partners do not. So, if eastward short Rossby waves are reflected at an eastern boundary, they will be reflected as westward (long) Rossby waves. If westward long waves are reflected at the western boundary, they will become eastward short waves.

Now, Pedlosky noted that if there is a process which selects to retain long waves over short ones, the wave activity will tend to be most pronounced near the western boundary. For example, in the presence of lateral friction, the shorter waves will 
be preferentially dissipated in a time $O\left(\delta_{M}^{-3} k^{-2}\right)$. If one considers that these waves propagate with zonal group velocity $C_{g} \approx \frac{1}{k^{2}}$, then short waves generated at the western boundary will only propagate a distance $O\left(\delta_{M}\right)$ away from the boundary. Similarly, even though bottom friction isn't scale selective, the distance traveled by short waves in the dissipative time is $O\left(\delta_{S}\right)$. Thus, the length scales of the Munk and Stommel boundary conditions reappear in Pedlosky's model.

All of the linear theories can be brought together by noting that the vorticity equation is not only an advective equation for the vorticity following the fluid parcel, but it is also a flux equation for the point-by-point vorticity. Thus, it describes where, in the Eulerian framework, the vorticity is supplied, transported, and removed and by what mechanism.

The $\beta$-flux, $\psi \hat{\mathbf{x}}$, is proportional to $\hat{\mathbf{x}}$, so it is always an east-west flux. Whether it is to the east or the west is determined by where the boundary conditions on the streamfunction are set, i.e., by the sign of $\psi$. If the streamfunction is set to zero in the east, following Sverdrup (1947), then the $\beta$-flux will be westward for negative $\psi$ and eastward for positive $\psi$. In a Sverdrup interior, the eastward flux, or to put it more sensibly, the westward flux of negative vorticity is caused by a negative vorticity input from the wind in the basin interior (as is the case in the subtropical gyre). A westward flux of positive vorticity is forced by a positive vorticity input in the basin interior.

Whatever sign of vorticity is fluxed to the west by the $\beta$-term, it cannot leave the basin as the $\beta$-flux must vanish at an impermeable boundary. Thus, it must diverge before reaching the boundary. Each linear theory presents a flux divergence to balance the $\beta$-flux divergence-bottom friction, lateral friction, or time-derivative. All of these balancing flux divergences exhibit symmetry upon switching the sign of $x$, while the $\beta$-term is anti-symmetric upon switching the sign of $x$. Thus, there is an intrinsic distinction in the vorticity equation between east and west, and a balance of the flux divergences can only be struck with a boundary current in the west. 
Similar ideas using vorticity fluxes will be used throughout the dissertation to illustrate the physical mechanisms responsible for the collection of vorticity in particular locations.

\section{Nonlinear Solutions: Returning to Symmetry}

Charney (1955) proposed a different kind of theory for the presence of boundary currents in the ocean. Since the ocean is relatively inviscid, it seemed to Charney that the friction was playing too much of a role in the theories of westward intensification. Charney's solution was to propose an entirely inviscid model which solved a model similar to

$$
\nabla \cdot\left(\hat{\mathbf{x}} \psi-\hat{\mathbf{x}} \delta_{I}^{2} \frac{\partial \psi}{\partial y} \nabla^{2} \psi+\hat{\mathbf{y}} \delta_{I}^{2} \frac{\partial \psi}{\partial x} \nabla^{2} \psi\right)=0
$$

This solution was intended to apply only near the boundary, and the Sverdrup solution would hold in the interior. ${ }^{2}$

Interpreted in the Lagrangian framework, this equation states that the absolute vorticity $\delta_{I}^{2} \nabla^{2} \psi+y$ is conserved following a fluid parcel It is therefore automatically solved so long as a single-valued function exists between $\psi$ and $\delta_{I}^{2} \nabla^{2} \psi+y$. Charney assumed that the boundary current would appear on the west and used the $\psi_{I}$ from the Sverdrup solution to determine the relationship as the flow entered the boundary current. Then, by insisting that $\psi=0$ at the boundary, he could reuse the function between $\psi$ and $\delta_{I}^{2} \nabla^{2} \psi+y$ to generate a boundary layer solution, which turns out to have a width $\delta_{I}$.

The problem with Charney's solution is that it only works so long as the function between $\psi$ and $\delta_{I}^{2} \nabla^{2} \psi+y$ remains single-valued, yet in the basin interior the absolute vorticity is nearly equal to $y$ (as the relative vorticity is negligible). If the fluid is to enter the boundary current at a small value of $y$ and leave it at a large value of

\footnotetext{
${ }^{2}$ Charney did not use the fixed-depth approximation, so the theory presented here differs from his, but is the corresponding solution in the homogeneous model.
} 
$y$, the function between $\psi$ and $\delta_{I}^{2} \nabla^{2} \psi+y$ can not be single-valued. Making matters worse, Greenspan (1962) was able to prove that no steady inertial theory could match smoothly to the interior in the region where the fluid exits the boundary current.

Restating the problem with Charney's model in terms of the balance of Eulerian fluxes, the $\beta$-flux divergence in the boundary layer is balanced by the constant import of relative vorticity advected from the interior. When the fluid leaving the boundary current is advected toward the interior, the import becomes an export and the interior solution can no longer be matched.

Other competing inertial theories to Charney's arose, such as that of Carrier and Robinson (1962) and Morgan (1956), but they were also incomplete.

In a result which is referred to a number of times in this dissertation, Fofonoff (1954) was able to find a complete solution, but for free modes instead of wind-driven solutions. His approach was similar to that of Charney, except since he didn't have to match to an interior solution, he was free to choose the function relating $\psi$ and $\delta_{I}^{2} \nabla^{2} \psi+y$ as he pleased, so long as it was single-valued. He chose a linear relationship and was able to produce free solutions.

However, a completely inertial theory like that of Fofonoff (1954) or Carrier and Robinson (1962) can never accept a constant input of vorticity from the wind, because none of the fluxes in an inertial theory-time-dependent, $\beta$-flux, or advection of relative vorticity flux-can ultimately remove vorticity from the basin. These fluxes can only move the vorticity around within the basin. Thus, an equilibrium can never be achieved.

Another problem with any of the purely inertial steady-state theories is that they do not distinguish east from west. Note that in Charney's equation 1.13, all of the terms change sign when $x$ changes to $-x$. Thus, these theories are incapable of distinguishing between a western and an eastern boundary layer; any solution which works with $x$ will also work with $-x$. The steady-state inertial theories do distinguish north from south, but they do not break the mirror symmetry to distinguish 
east from west. In the homogeneous model, one of the following terms is required to distinguish western boundary currents from eastern: time-dependence (Pedlosky (1965a)), bottom friction (Stommel (1948)), or lateral friction (Munk (1950)).

Perhaps one can fix the Charney solution and at the same time force the boundary currents to the west. Suppose only a small amount of friction is used. Then the relative vorticity of the fluid could change as it propagated through the boundary current so that the problem with returning to the interior flow might disappear. Simultaneously, the introduction of friction would distinguish east from west.

With this in mind, we turn to the problem of the inertial Munk and inertial Stommel solutions. In these solutions, the friction and the advective terms are considered simultaneously. Once the exact conservation of vorticity is broken, however, the hope of analytic treatments of equations containing the nonlinear advective terms drops precipitously. For this reason, our review of westward intensification switches to computational results.

Once the effects of both inertial and frictional terms are considered simultaneously, it is helpful to provide a dimensionless constant which weighs their relative importance. When lateral friction and inertia are compared, the Reynolds number is this constant. It relates the typical scale of the inertial terms to the typical scale of the frictional ones. Since most of the inertial activity here goes on in the western boundary layer, it is the ratio of the terms in the western boundary region that is important. There the scaling of Charney's solution gives the following: $\frac{\partial \psi}{\partial x} \approx \delta_{I}^{-1}$, and $\frac{\partial \psi}{\partial y} \approx 1$. This scaling results from the width of the boundary layer being Charney's width $\left(\delta_{I}\right)$, and the length of the current being the basin dimension (which is 1). Recall that the nondimensional wind forcing is chosen to give a Sverdrup solution with $\psi$ of order 1. Examining these scales, we find:

$$
\begin{gathered}
O(\nabla \cdot(\hat{\mathbf{x}} \psi)) \approx \delta_{I}^{-1} \\
O\left(\nabla \cdot\left(-\hat{\mathbf{x}} \delta_{I}^{2} \frac{\partial \psi}{\partial y} \nabla^{2} \psi\right)\right) \approx \delta_{I}^{-1}
\end{gathered}
$$




$$
\begin{gathered}
O\left(\nabla \cdot\left(\hat{\mathbf{y}} \delta_{I}^{2} \frac{\partial \psi}{\partial x} \nabla^{2} \psi\right)\right) \approx \delta_{I}^{-1} \\
O\left(\nabla \cdot \delta_{M}^{3} \nabla \nabla^{2} \psi\right) \approx \frac{\delta_{M}^{3}}{\delta_{I}^{4}} . \\
\operatorname{Re} \equiv O\left(\frac{\nabla \cdot\left(\hat{\mathbf{x}} \psi-\hat{\mathbf{x}} \delta_{I}^{2} \frac{\partial \psi}{\partial y} \nabla^{2} \psi+\hat{\mathbf{y}} \delta_{I}^{2} \frac{\partial \psi}{\partial x} \nabla^{2} \psi\right)}{\nabla \cdot \delta_{M}^{3} \nabla \nabla^{2} \psi}\right) \approx \delta_{I}^{3} / \delta_{M}^{3}
\end{gathered}
$$

The Reynolds number will be very important in this dissertation.

\section{Friction in Numerical Models}

In order to get a boundary layer of the correct width, Munk's model requires that the viscosity be many orders of magnitude larger than the molecular viscosity of water. The kinematic viscosity of water is about $1 \cdot 10^{-6} \mathrm{~m}^{2} / \mathrm{s}$ for ocean surface temperatures. The nondimensional Munk width used here is $\delta_{M}=\left(A_{H} /\left(\beta L^{3}\right)\right)^{1 / 3}$, where $A_{H}$ is the eddy viscosity. Thus, in order to have a $100 \mathrm{~km}$ Munk boundary

current in a $10,000 \mathrm{~km}$ basin with $\beta=2 \cdot 10^{-11}(\mathrm{~ms})^{-1}$, the value of $A_{H}$ needs to be approximately $2 \cdot 10^{4} \mathrm{~m}^{2} / \mathrm{s}$, ten orders of magnitude larger than the molecular viscosity. The reason that this is allowable is that the larger viscosity is intended to represent not molecular viscosity, but eddy viscosity. If an estimate of eddy viscosity is made from observations, then this larger value is more reasonable. For example, LaCasce and Bower (2000) determined that the dispersion of subsurface floats in the ocean is consistent with an eddy diffusivity of floats in the range: $2 \cdot 10^{3}\left(\mathrm{~m}^{2} / \mathrm{s}\right)$ to $2 \cdot 10^{4}\left(\mathrm{~m}^{2} / \mathrm{s}\right)$ for floats separated by greater than $100 \mathrm{~km}$. If the vorticity of the fluid is transported along with the floats then the eddy viscosity would be equal to the eddy diffusivity of the floats.

Eddy viscosity is a simplistic way to represent the effects of unresolved eddies into a model without dealing with the eddying motions themselves; it is a parameterization of the eddies. Eddy viscosity is a good approximation when the motions of the eddies are relatively homogeneous and isotropic and have significantly smaller scales in space and time than the physics being explicitly modeled.

To illustrate the meaning of eddy viscosity and the assumptions it involves, I will 
consider two approaches to the problem: top-down and the bottom-up.

For the top-down approach, consider the running average of equation 1.1 over a spatial scale $\Delta x, \Delta y$ and a time scale $\Delta t$. We can then define the slowly-changing part of any of the fields $f$ as:

$$
\begin{gathered}
\bar{f} \equiv \frac{1}{\Delta x \Delta y \Delta t} \int_{x}^{x+\Delta x} \int_{y}^{y+\Delta y} \int_{t}^{t+\Delta t} f \mathrm{~d} t \mathrm{~d} y \mathrm{~d} x \\
f^{\prime} \equiv f-\bar{f}
\end{gathered}
$$

We can now take the average of each term in equation 1.1. Most of the terms will be of the form:

$$
\begin{gathered}
\overline{\frac{\partial g}{\partial t}}=\frac{1}{\Delta x \Delta y \Delta t} \frac{\int_{x}^{x+\Delta x} \int_{y}^{y+\Delta y} \int_{t}^{t+\Delta t} \frac{\partial g}{\partial t} \mathrm{~d} t \mathrm{~d} y \mathrm{~d} x}{,} \\
\overline{\nabla \cdot \mathbf{V}}=\frac{1}{\Delta x \Delta y \Delta t} \int_{x}^{x+\Delta x} \int_{y}^{y+\Delta y} \int_{t}^{t+\Delta t} \nabla \cdot \mathbf{V} \mathrm{d} t \mathrm{~d} y \mathrm{~d} x
\end{gathered}
$$

Now, if the integral of the eddy field is such that over the time and space scales of the integration its integral tends to zero, then the derivatives in the preceding equations and the integrals approximately commute, just as if the averages were not over space and time but over an ensemble of different realizations of the flow.

If there were no mean flow and the domain were infinite, then the averages could be taken in the limit that $\Delta x$ and $\Delta t$ go to infinity. If these averages converge, then the fields are ergodic. Without a mean flow, the ergodic eddy fields' statistics are stationary in time and homogeneous in space.

Here, in order to make the averaging formally correct we require the stronger constraint that these integrals converge rapidly. That is, there must be a separation of scales between the eddy field and the mean field. If there is a scale separation, then the integrals and the averages commute. So, for example if equation 1.1 were considered to be the true dynamics if all scales could be resolved, then it can be 
written in Reynolds-averaged form:

$$
\frac{\partial \nabla^{2} \bar{\psi}}{\partial t}+\frac{\partial \bar{\psi}}{\partial x}+\delta_{I}^{2} J\left(\bar{\psi}, \nabla^{2} \bar{\psi}\right)+\delta_{I}^{2} \overline{J\left(\psi^{\prime}, \nabla^{2} \psi^{\prime}\right)}-\nabla \cdot \delta_{M}^{3} \nabla \nabla^{2} \bar{\psi}+\delta_{S} \nabla^{2} \bar{\psi}=\overline{w_{E}}
$$

The operator $J$ is the Jacobian, which is defined as $J(A, B)=\frac{\partial A}{\partial x} \frac{\partial B}{\partial y}-\frac{\partial B}{\partial x} \frac{\partial A}{\partial y}$ for arbitrary $A$ and $B$. This equation is just like equation 1.1, except instead of acting on the total field $\psi$, it acts on the slowly-evolving field $\bar{\psi}$ and there is a new eddy term proportional to $\overline{J\left(\psi^{\prime}, \nabla^{2} \psi^{\prime}\right)}=\nabla \cdot\left(\overline{\mathbf{u}^{\prime} \nabla^{2} \psi^{\prime}}\right)$.

The bottom-up approach estimates the eddy term from the top-down approach. This approach is related to the one without a slowly-evolving flow first proposed by Taylor (1921). To begin, we consider a small region in a coordinate frame which moves along with the mean velocity over the small region and averaged with time so that $\bar{u}=0$. Then, we consider the motion of a parcel of fluid moving turbulently beginning at the origin of these coordinates, so that its location and displacement are both given by $\mathbf{x}^{\prime}(t)$. If we consider a tracer in the fluid which is nearly conserved, $q$ (with a slowly-varying component $\bar{q}$ and perturbations from that slowly-varying component $a^{\prime}$ ) then we expect that as this parcel moves about it retains nearly the same amount of this tracer. As pointed out in the last section, the absolute vorticity is a nearly conserved quantity. Thus,

$$
q\left(\mathbf{x}^{\prime}, t\right) \approx q\left(\mathbf{x}_{\mathbf{0}}=0, t=0\right)
$$

If this parcel possesses the tracer concentration typical of its initial location, then the size of its tracer anomaly at its new location can be estimated:

$$
\begin{aligned}
& q\left(\mathbf{x}^{\prime}, t\right) \approx q\left(\mathbf{x}_{\mathbf{0}}=0, t=0\right) \approx \bar{q}\left(\mathbf{x}_{\mathbf{0}}=0, t=0\right) \\
& q^{\prime}\left(\mathbf{x}^{\prime}, t\right)=q\left(\mathbf{x}^{\prime}, t\right)-\bar{q}\left(\mathbf{x}^{\prime}, t\right) \approx-\mathbf{x}^{\prime}=\nabla \bar{q}(\mathbf{x}, t)
\end{aligned}
$$

The last step is a truncated Taylor expansion. This validity of performing and trun- 
cating this expansion relies on two assumptions. First, the terms involving higher derivatives of $\bar{q}$ are neglected because $\bar{q}$ is considered to be changing slowly enough across the domain to be linearized. Thus, again there must be a clear scale separation between the scale of variation of $\bar{q}$ and the eddy scale, $\mathrm{x}^{\prime}$. Second, $x^{\prime}$ is considered to be independent of $q$, that is, $q$ should be a passive tracer. ${ }^{3}$

The eddy flux of tracer is therefore estimated by

$$
\mathbf{u}^{\prime} q^{\prime} \approx-\mathbf{u}^{\prime}\left(\mathbf{x}^{\prime} \cdot \nabla \bar{q}\right)
$$

If we assume that the eddy field is isotropic and make the simplifying assumption that there are no cross-correlations in the displacements, then ${ }^{4}$

$$
\mathbf{u}^{\prime} q^{\prime} \approx-\frac{1}{2} \frac{\mathrm{d}}{\mathrm{d} t}\left(x^{\prime 2}\right) \nabla \bar{q}
$$

Taking the average,

$$
\overline{\mathbf{u}^{\prime} q^{\prime}} \approx-\frac{1}{2} \frac{\mathrm{d}}{\mathrm{d} t}\left(\overline{x^{\prime 2}}\right) \nabla \bar{q}
$$

An important assumption has been that the eddy scale is separated from the slowlyevolving scale. Now, we are in a position to be more precise as to the meaning of the eddy scale. If the eddies are somewhat random in their movements, we expect that when the eddies are allowed to evolve over a large enough region and for a large enough time, the rate of displacement will become constant, that is,

$$
\lim _{\left(t, \mathbf{x}^{\prime}\right) \rightarrow \infty}\left[\frac{1}{2} \frac{\mathrm{d}}{\mathrm{d} t}\left(\overline{x^{\prime 2}}\right)\right]=\kappa
$$

Now, once again, we require the scale separation of the eddies and the slowly-evolving

\footnotetext{
${ }^{3}$ Although there can be a relationship between $q$ and $u, q$ must be passive at least the functional relationship relating them doesn't change the form or the Taylor expansion given above.

${ }^{4}$ This step can be performed without neglecting the cross-correlations, but tensors must be used to do so. Thus, for simplicity I avoid this step.
} 
flow; this limit must converge before the slowly-evolving scales are reached.

A similar but more extensive presentation of the meaning of eddy viscosity is given in Tennekes and Lumley (1972). More rigorous presentations are to be found in Batchelor (1952) and Batchelor (1960).

Of course, the eddy viscosity model is flawed; eddies in fluids typically do not have clearly separate spatio-temporal scales from the mean, and vorticity is not a passive tracer. Furthermore, the presence of boundaries and structure in the time-mean flow places the assumption of turbulent isotropy and homogeneity in doubt.

To minimize the flaws of eddy viscosity, it is often the goal of numerical modelers to resolve as many of the eddies as possible. That is, to make the eddy viscosity small and explicitly deal with as many of the motions that result as possible. Ocean modelers typically produce large eddy simulations. That is, they use a eddy viscosity which is larger than the molecular viscosity, but the appropriate eddy viscosity is governed by the scale of motions explicitly resolved. If this scale changes because of a change in resolution, more and more of the motions are explicitly calculated, and so less and less of the eddies are parameterized. Thus, the estimates of eddy diffusion coefficient in the ocean that I have cited from LaCasce and Bower (2000) should be interpreted as close to the maximum eddy viscosity to use sensibly, because these numbers are found in the limit of very long time averages and very large spatial averages and therefore correspond to a model for a very slowly-evolving state with most of the eddies parameterized.

One further point should be made when discussing friction in numerical models: numerical dissipative errors.

The standard approach to solving partial differential equations is to discretize using some approximation of the continuous field at points on a fine grid (finite differences) or approximate the average of the continuous field within little cells (finite volumes). The operators on the continuous fields are estimated in the discretized model by arranging to find as many of the terms in a Taylor series expansion of 
the result of the operators using the values at the grid points or the averages over the volumes. The order of the discretization is the order to which the Taylor series expansion is satisfied.

Interestingly, some of the errors that result from truncating the Taylor series expansion act as though the discrete representation is a representation of the continuous fluid with more viscosity or hyperviscosity than is explicitly incorporated into the discretized equations. These dissipative errors increase as the grid resolution decreases.

Thus, when a model is set up in finite differences or finite volumes and solved, the model may act in a more dissipative manner than desired. As the Reynolds number increases along with the demands on the model resolution, these errors will become larger and larger, even before the onset of numerical instability due to insufficient resolution. The primary model used in this dissertation is spectral, which avoids dissipative errors but has other sorts of errors instead (see section C.5 for a discussion of these errors)

However, dissipative errors are prevalent in many calculations in publication. Errors are an inevitable consequence of numerical modeling. Thus, when a calculation is purported to be operating at a particular Reynolds number, but the frictional length scales are comparable to or smaller than the grid scale, it is likely that the errors in the discretization make the numerical model closer to a representation of a more viscous model than a properly-resolved model using the explicit value of the viscosity. This should be kept in mind when a result is presented from a model with a particular explicit viscosity and marginal resolution.

\section{Failure at Moderate Reynolds Number}

Before going on to two-dimensional calculations, it is the appropriate point to mention the results of Ierley and Ruehr (1986). They solved a one-dimensional approximation to the inertial Munk model in the boundary layer numerically, and showed that the difficultly in connecting to the interior flow in the exit region that troubled Charney's 
model persists even when friction is present, once the Reynolds number is greater than one. Apparently, the one-dimensional boundary-layer approach is too limited to capture the full dynamics.

We now turn to calculations of the inertial Munk and inertial Stommel model in two dimensions. One school of thought has sought to simplify by keeping all of the time-dependent phenomena parameterized by the eddy viscosity. These researchers calculate steady-state solutions of the inertial Munk and inertial Stommel models.

Some interesting results have been found by calculating steady-state solutions. First, as demonstrated convincingly by Ierley (1987) (and further studied by Cessi et al. (1987) and Ierley and Young (1991a)) at the same Reynolds number as the viscoinertial solutions of lerley and Ruehr (1986) break down, a recirculation gyre forms in the exit region from the boundary current. Since a recirculation is a two-dimensional phenomenon, it need not obey the one-dimensional boundary-layer approximations used by Ierley and Ruehr (1986). Also, Ierley and Sheremet (1995) found that, because of the nonlinearity of the inertial terms, more than one solution is possible for the inertial Munk problem with the same forcing.

However, a troubling effect occurs in these steady-state numerical calculations. As the Reynolds number becomes moderately large, the solution strength increases rapidly and apparently without bound. This effect was first pointed out in an inertial Stommel model by Veronis (1966b). Ierley and Sheremet (1995) fully explored this result in the inertial Munk model. Figures 1-1 and 1-2 show this behavior in steadystate solutions found with the method described in Appendix B.

A change in circulation strength with a change in viscosity is not unexpected behavior for a simple physical system. If the friction is removed while the forcing is kept steady, then of course any system with inertia would behave in this way. The troubling aspects are that the inertia dominates at such a low Reynolds number, many times smaller than the Reynolds number estimated to be appropriate for ocean models and the real ocean, and that westward intensification is lost as the circulation 

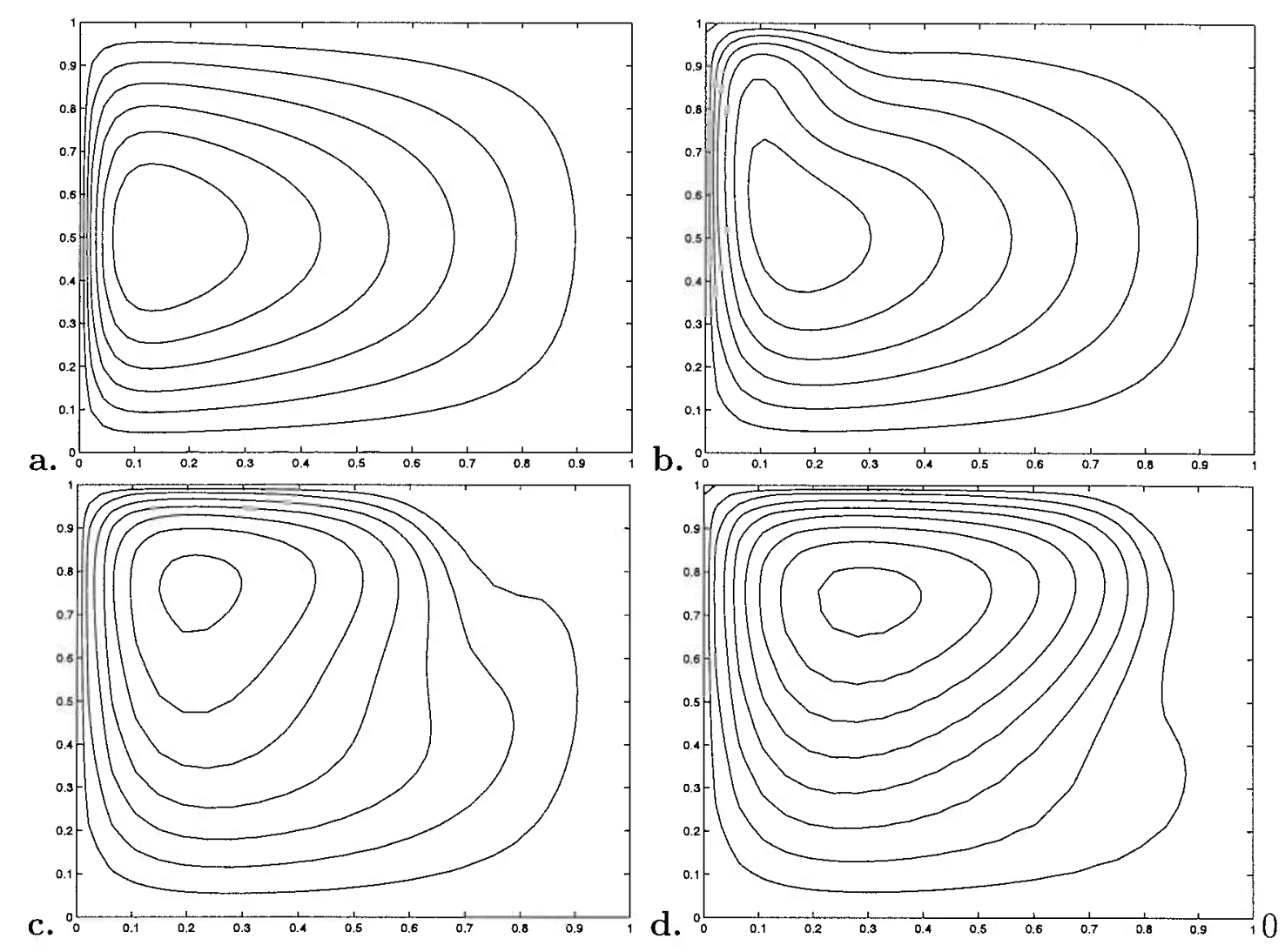

Figure 1-1: (a-d) contour the steady-state streamfunction for the homogeneous model with bottom friction only. (a) shows the $\delta_{I}=0, \delta_{S}=0.04$ calculation and has $\max (\psi)=0.70$. (b) shows the $\delta_{I}=0.04, \delta_{S}=0.04$ calculation and has $\max (\psi)=$ 0.68. (c) shows the $\delta_{I}=0.08, \delta_{S}=0.04$ calculation and has $\max (\psi)=0.73$. (d) shows the $\delta_{I}=0.1, \delta_{S}=0.04$ calculation and has $\max (\psi)=0.83$. The contour interval is 0.1 in units where 1 is the maximum of the Sverdrup solution.

strength increases. Although a fairly large recirculation gyre may be consistent with ocean observations (see Sheremet (2002) for a discussion), it is clear that the the oceans are western-intensified on the large scale and that figures $1-2 \mathrm{c}$ and $1-2 \mathrm{~d}$ are therefore unrealistic.

Of course, the missing ingredient might be the effect of eddies. It is sensible that since the large value of the viscosity was intended as an eddy viscosity, lowering it would require and induce the presence of unstable, time-dependent motions through the natural instability of fluids. These eddies might be able to replace the effects of 

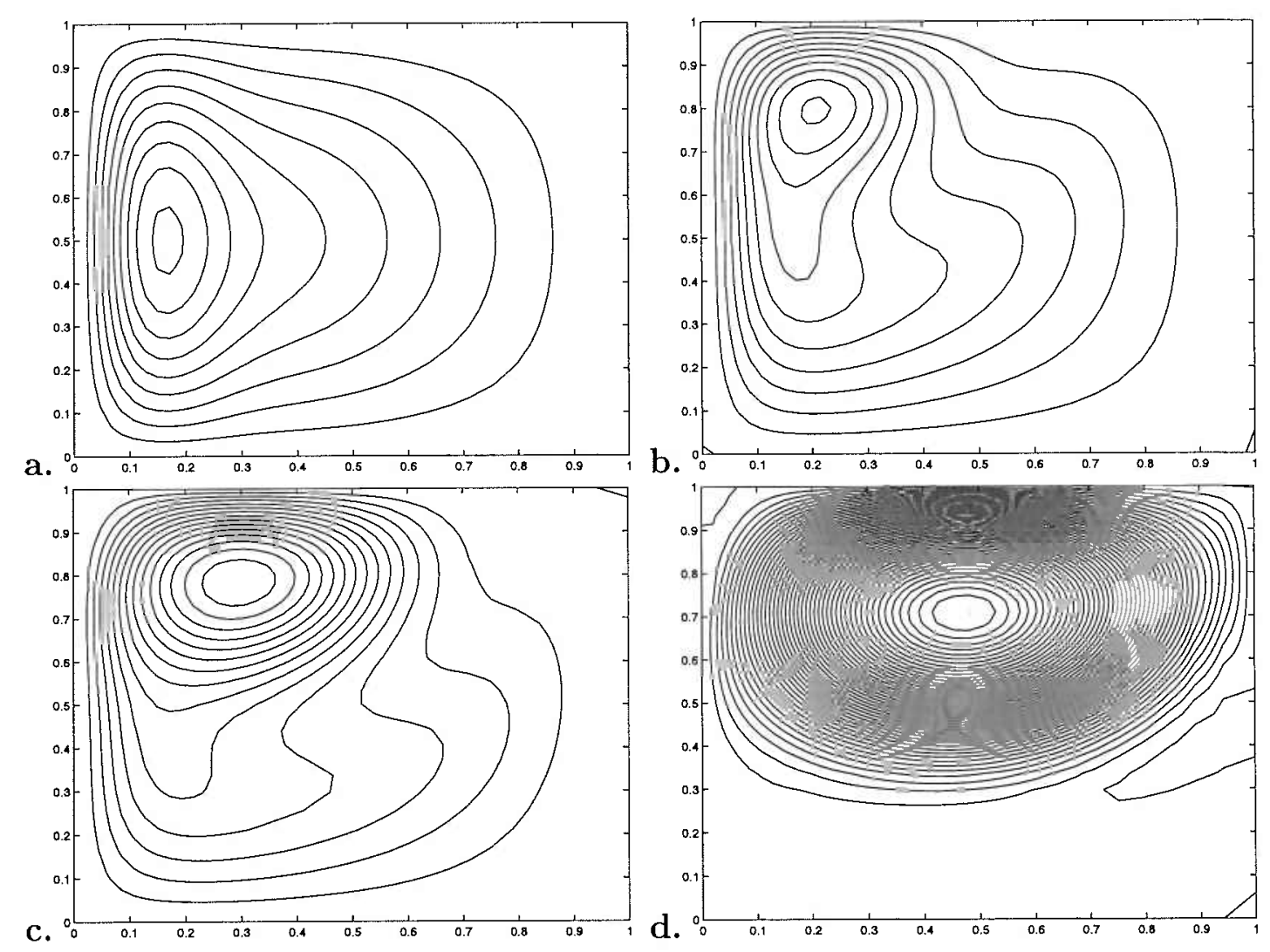

Figure 1-2: (a-d) contour the steady-state streamfunction for the homogeneous model with lateral friction only. The frictional boundary conditions are no-slip on the eastern and western boundaries and slip on the others. (a) shows the $\delta_{I}=0, \delta_{M}=0.05$ calculation and has $\max (\psi)=0.93$. (b) shows the $\delta_{I}=0.05, \delta_{M}=0.05$ calculation and has $\max (\psi)=1.02$. (c) shows the $\delta_{I}=0.0625, \delta_{M}=0.05$ calculation and has $\max (\psi)=1.5$. (d) shows the $\delta_{I}=0.08125, \delta_{M}=0.05$ calculation and has $\max (\psi)=4.5$. The contour interval is 0.1 in units where 1 is the maximum of the Sverdrup solution.

lowering the eddy viscosity as the Reynolds number increased, as is crudely the case in large eddy simulations of homogeneous, isotropic 2-dimensional turbulence (for example, McWilliams et al. (1994)). Figure 1-3 shows the result of such a calculation for the homogeneous model. This result agrees with other studies such as that of Kamenkovich et al. (1995).

In this dissertation, I refer to the solutions such as that in figure 1-3d as inertiallydominated. Some authors refer to the appearance of inertial-dominance at modest 

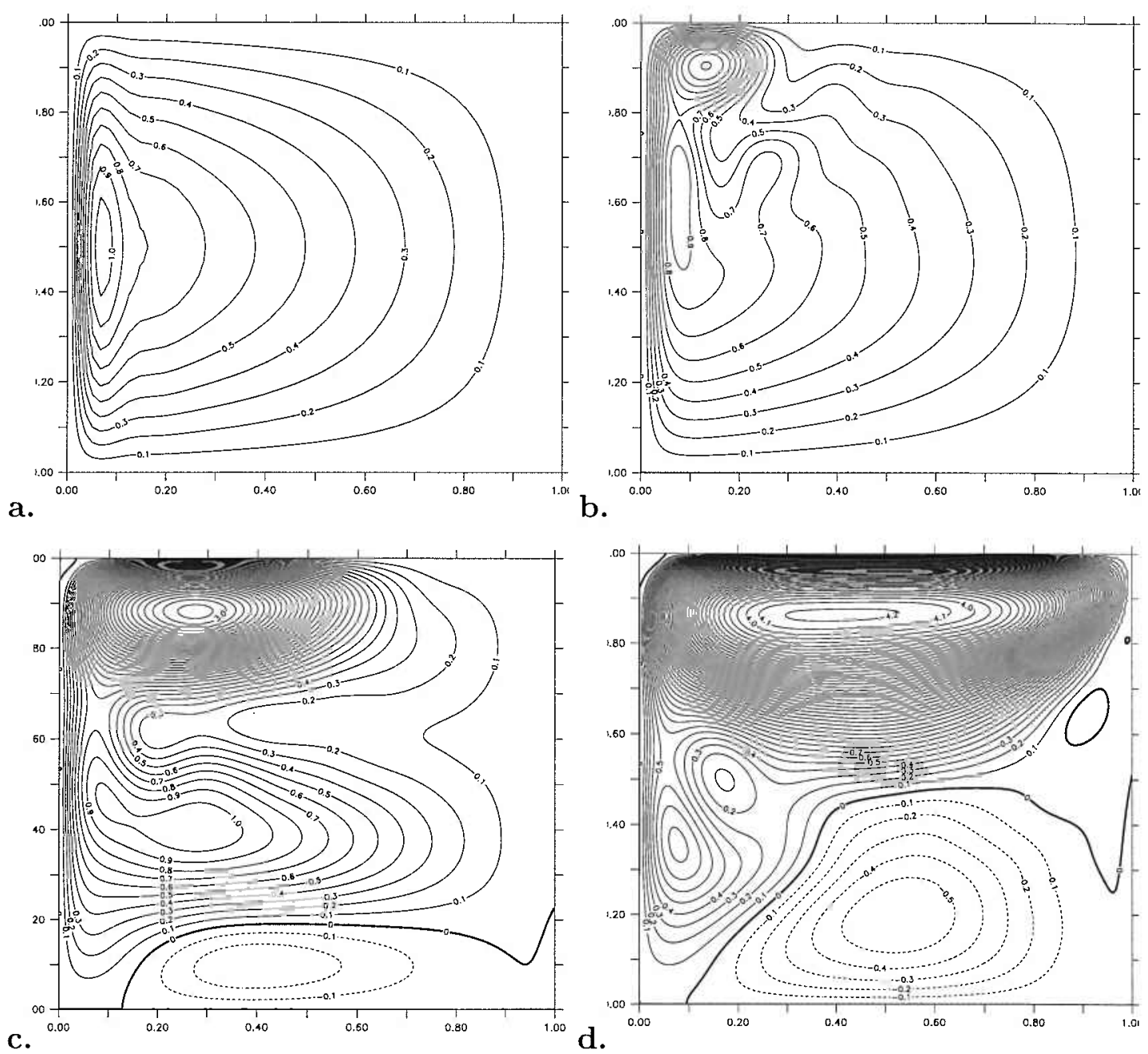

Figure 1-3: (a-d) contour the time-mean streamfunction for the homogeneous model with lateral friction only. The frictional boundary conditions are no-slip on the eastern and western boundaries and slip on the others. The solution is calculated using the Chebyshev-polynomial time-dependent integrator described in Appendix C. (a) shows the $\delta_{I}=0, \delta_{M}=0.02$ calculation and has $\max (\bar{\psi})=0.93$. (b) shows the $\delta_{I}=0.02$, $\delta_{M}=0.02$ calculation and has $\max (\bar{\psi})=1.31$. (c) shows the $\delta_{I}=0.02, \delta_{M}=0.01387$ calculation and has $\max (\bar{\psi})=3.15$. (d) shows the $\delta_{I}=0.02, \delta_{M}=0.0117$ calculation and has $\max (\bar{\psi})=4.23$. The contour interval is 0.1 in units where 1 is the maximum of the linear Sverdrup solution. 
Reynolds numbers as the problem of inertial runaway. However, that term will rarely be used here because it implies that no equilibrium is reached. These calculations do reach an equilibrium, it is just at a much higher mean circulation strength and less westward intensified than desired.

One might suppose that perhaps the inertial domination occurs because the homogeneous model is two-dimensional. The important effects of baroclinic instability are neglected here. However, even when a model which incorporates the third dimension using layers of fluid stacked on top of each other is used inertial-domination is observed at moderate Reynolds number (for example, Holland and Lin (1975)).

Why is it unacceptable that the circulation strength increases so rapidly in this model? Presumably it indicates the readily apparent fact that there are physical processes missing from the model. From a historical perspective, the reason Stommel (1948) began all of this research was to explain why the intensification was toward the west. As shown in figure 1-3d, the inertially-dominated solutions are not particularly western-intensified. This lack of westward intensification can be easily understood by appeal to the flux arguments above. When the circulation strength increases

sufficiently, the westward flux of vorticity of the $\beta$-term is swamped by the eastward flux of vorticity of the inertial terms exiting the boundary current. One expects that with higher Reynolds number, the solution will become completely symmetric in the east-west direction as the frictional terms are no longer large enough to break the symmetry.

\subsubsection{What Sets the Wind-Driven Circulation Strength?}

A simple model is very likely to become inertially-dominated as the friction decreases; just as a bicycle wheel would spin faster and faster if the friction were greased away with a constant torque applied. But, the ocean is not a simple model, it is a complex physical system, and it can change its response to forcing in many ways. Obviously, it responds in such a way that it does not require enormous circulation strength at 
small viscosity nor does it possess large viscosity.

Ultimately, of course, the friction will have to play a role in setting the circulation strength, as the ocean constantly receives an input of vorticity forced by the wind. In a model of only oceanic processes that conserve energy and vorticity, the quantity of each would continue to build indefinitely without the effects of friction. As mentioned above, perhaps it is not necessary for the friction to reach the molecular viscosity, but at least, it should be clear what processes are represented by an eddy viscosity, and the parameterization should be replaceable with resolved physics at least in principle. However, can a model be made which prevents the enormous increase in the timemean flow of the wind-driven ocean which is relatively inviscid, simple, and westernintensified? Such a model is presented in Chapter 2 of this dissertation.

Interestingly, the Sverdrup solution appears to set the circulation strength without considering friction. How is this possible? The Sverdrup relation, though it can determine circulation strength, in order to do so, it requires a passive boundary current. That is, the reason that the Stommel, Munk, and Charney models were able to attach to the Sverdrup flow was that they did so passively. Passive attachment requires that the necessary mass flow and the necessary removal of vorticity is performed. The Stommel and Munk models lack advection of relative vorticity, so the removal of vorticity is no problem. Also, since the solutions are linear, if the solution exists at one forcing strength it exists at all forcing strengths there is no limit to the mass transport that these linear solutions can carry. The Charney model can not reattach passively, so although it removes no vorticity from the flow, it need not because it doesn't describe a complete circulation.

Thus, the necessary criterion in for the simple model has to do with reattachment of the boundary layer to the interior flow. In order to do this, the boundary current must be able to transport enough mass and remove enough vorticity in the region of the boundary layer. If not, the reattachment is doomed to fail. As we will see, the formation and strength of recirculation gyres in the exit region indicates the mismatch 
in rejoining the interior flow. Most of the calculations in figures 1-1, 1-2 and 1-3 do not have a passive boundary layer. Even when they are not inertially-dominated they do possess a recirculation gyre which increases the maximum of the streamfunction some degree above 1, the Sverdrup solution maximum.

The requirement of a change in vorticity in a relatively inviscid boundary layer is reminiscent of the study by Edwards and Pedlosky (1998). They modeled the crossequatorial flow of a deep western boundary current. Thus, the flow was input with a particular planetary vorticity and removed with a different vorticity. As the Reynolds number of their boundary current increased, it became increasingly difficult for the vorticity transformation to occur. We will see that the boundary current found in the calculations here dynamically resemble the boundary current found by Edwards and Pedlosky (1998).

\subsubsection{The Role of Vorticity}

In most of this dissertation, the analysis will closely examine the dynamics of vorticity. Not only are the dynamics of absolute vorticity mathematically convenient to analyze, but the amount of absolute vorticity directly addresses the issue of the strength of the circulation. Of course, vorticity is expected to play an important role in a rapidlyrotating fluid.

Consider a primarily horizontal time-mean circulation in a closed basin. Suppose that there are no sources or sinks of mass and that the fluid is incompressible. Then, the depth-integrated transport streamfunction will form nested circles of circulation. The transport around each extremum of the streamfunction is governed by the magnitude of the extremum. What sets the magnitude of these extrema? Since the streamfunction is set to zero on the boundary, it will be the gradient of the streamfunction (not coincidentally, the gradient of the streamfunction is the depth-integrated velocity). By integrating the relative vorticity within a particular streamline and using 
the divergence theorem, we find

$$
\frac{\iint \nabla^{2} \psi \mathrm{dA}}{\oint \mathrm{dl}}=\frac{\oint \nabla \psi \cdot \hat{\mathrm{n}} \mathrm{l} l}{\oint \mathrm{dl}}
$$

That is, the average gradient of the streamfunction across a particular streamline is the amount of relative vorticity contained within that streamline per unit streamline length.

Thus, if the wind input inside of a particular streamline is allowed to build, the gradient of the streamfunction will increase and the circulation strength will increase.

Likewise, we can consider the effect of vorticity trapped between two streamlines. If streamline 2 is nested inside streamline 1 , then integrating the vorticity over the area between the streamlines, we find

$$
\left|\iint \nabla^{2} \psi \mathrm{dA}\right|=\left|\oint_{2} \mathbf{u} \cdot \mathrm{d} \mathbf{l}-\oint_{1} \mathbf{u} \cdot \mathrm{d} \mathbf{l}\right|=\left|<u_{2}>L_{2}-<u_{1}>L_{1}\right|
$$

The angle brackets denote the average value of the velocity around that streamline and $L_{1}$ and $L_{2}$ are the lengths of each streamline. ${ }^{5}$ So, if vorticity builds up in the region between two streamlines without changing the position of the streamlines, the difference in average velocity around the streamlines increases. Thus, if vorticity builds up between two streamlines, the flow around the inner nested streamlines moves faster and faster. It is similar reasoning which gives the circulation, $\Gamma$, its name.

$$
\Gamma \equiv \iint \nabla^{2} \psi \mathrm{dA} \equiv \oint \mathbf{u} \cdot \mathrm{d} \mathbf{l}
$$

So, it is clear that the question of what controls the circulation strength can be put in terms of where the vorticity tends to pile up and how it is transported.

The $\beta$-flux of vorticity is always in the east-west direction, and the advective flux by the mean flow of time-mean relative vorticity is along the direction of the time-

${ }^{5}$ Absolute values are used to eliminate the complications of considering sign conventions and vorticity of either sign. 
mean velocity. In chapter 2 , it is demonstrated that neither of these fluxes can carry a net flux of vorticity across a time-mean streamline. In the homogeneous model, only the frictional fluxes and the eddy fluxes (that is, the advective flux of perturbation relative vorticity by the perturbation velocity) can carry vorticity across time-mean streamlines.

Therefore, the story of what controls the circulation strength is the story of how the frictional fluxes and the eddy fluxes can remove the vorticity input by the wind from within closed time-mean streamlines. A result which has a important impact on the dynamics of the homogeneous model is that the resolved eddies in this model cannot destroy vorticity or remove it from the basin as the eddy flux must vanish at impermeable boundaries. Thus, the interpretation of what friction parameterizes in this model needs revision from the ideas presented above.

\section{Internal Cancellation}

A different consideration in the control of the circulation strength is what happens when the wind's vorticity input is not of a single sign. If the wind provides positive vorticity in one region and negative vorticity in another, then internal cancellation of the vorticity could occur if the fluid arranges itself to communicate between these regions. This question has been addressed by many previous authors (for example, Harrison and Holland (1981), Marshall (1984), and Lozier and Riser (1990)).

Marshall (1984) suggests that one should not consider a single gyre, but two rotating in opposite directions, representing the sub-polar and subtropical gyres. This presents the possibility of internal cancellation of the wind's vorticity input. By intergyre vorticity exchange, the mean flow or eddies could ease the burden on the friction to remove the vorticity input by the wind. Chapter 4 will deal with this issue in detail. 


\subsection{Dissertation Goals and Choice of Model}

In Ierley and Sheremet (1995) it was suggested that the circulation does not, nor should it, saturate in the limit of vanishing lateral viscosity. The role of instability is simply to retard the increase in recirculation beyond realistic values. Barotropic instability alone, we argue, is insufficient to retard the increase in recirculation beyond realistic values. Baroclinic instability and internal gravity waves are obvious candidates for future investigation.

-Sheremet et al. (1997)

The goal of this dissertation is to address the reasons why the wind-driven circulation strength is what it is, and to describe some of the processes involved.

In order to address this goal, numerical models were developed. The primary model, which integrates the time-dependent homogeneous model (equation 1.1), was chosen for a number of reasons. What makes the model used here unique is that the viscosity is increased as the eastern and western boundaries are approached.

First, the model ties neatly into the tradition of models of westward intensification. Only in the spatial variations of viscosity does it vary from these models, so many of the lessons learned apply directly.

Second, the model is easily and efficiently implemented numerically. The only real difficulty numerically is accurately representing the derivatives necessary to evaluate the friction. As the viscosity decreases, the gradients become very large so that friction remains part of the solution. These gradients require very fine horizontal resolution as the viscosity decreases. However, this constraint is present in any high Reynolds number calculation, so common practices such as those given in Orszag and Israeli (1974) can be adapted.

Third, the vorticity dynamics of the model are simple. As mentioned, only eddy fluxes and frictional fluxes can carry vorticity across streamlines of the mean flow. 
Because the depth of the model is fixed, only absolute vorticity need be considered.

Fourth, the model possesses few parameters, so a large range of the possible perturbations can be explored. This is the driving idea behind what is likely the weakest point of the model: neglect of vertical and density structure, including the important effects of topography.

If an omnipotent being were to study what controlled the strength of the ocean circulation, her model of the ocean would solve the full three dimensional NavierStokes' equations at any resolution desired. It would have a diversity of phenomena and boundaries which are like those in the real ocean-rough-but adequately resolve the pockets of fluid contained in the nooks and crannies of the rough topography and coastlines. Potentially important effects, such as the time-dependence of the winds and the astronomical forcing of the tides, would be included. However, it would take the intellect of an omniscient to understand the results of such a model.

Instead, given the limited resources of computational power and finite intellect, the only course is to simplify as much as possible without neglecting the critical features which perform the control of the circulation strength. So, I have chosen the homogeneous model with boundary-enhanced viscosity.

This model is intended to represent the same idealized wind-driven circulation envisioned by Stommel and Munk, and makes similar approximations with one exception. As mentioned above in section 1.1.3, the eddies present in this model cannot remove vorticity from the basin. This is quite unlike the eddies, turbulence and waves operating at small scales in the ocean. There are many phenomena which can remove vorticity from the circulation near the boundaries or which will certainly strongly affect the eddy field only near the boundaries. Prime examples are bottom pressure interaction with topography (as recently proposed by Hughes and De Cuevas (2001)), eddy generation at a sloping bottom (as proposed by Hallberg and Rhines (2000)), and the breaking of internal gravity waves near topography. 
The homogeneous model is so simple, with its 2-dimensional circulation without topography or sloping boundaries, that the friction must play a dual role. It must represent both the eddy viscosity, i.e., the random mixing of the mean flow by unresolved eddies, and the removal of vorticity from the basin by all processes capable of performing this removal. The friction parameterizes the mixing by eddies by diffusing vorticity in the basin interior. The friction parameterizes the effects of interaction with the boundaries as it is the only mechanism for removal of vorticity from the basin. Furthermore, the use of friction requires more boundary conditions and tends to result in frictional boundary layers. In the homogeneous model, these effects of friction are parameterizations of the interaction of the large-scale flow in the real ocean with the boundary.

However, the use of a constant viscosity tacitly assumes constant intensity of the unresolved phenomena whose effects on the large scale circulation are parameterized by the friction. Clearly, since the friction must represent completely different phenomena near the boundary than it does in the basin interior, it is unfair to expect these processes to parameterize easily as a constant viscosity. Moreover, because of the simple topography and physics of the homogeneous model, the only part of the eddy viscosity which will become explicitly resolved as the Reynolds number increases are the barotropic eddies. All other unresolved parameterized phenomena will remain implicit in this model.

It is obviously true that the results here would be more convincing were the boundary processes resolved rather than parameterized by an increased viscosity. This dissertation is intended only as a first step to point out the importance of these processes and to determine their effect on the interior circulation. Later investigations can determine the precise nature of these phenomena. Also, once the effects on the interior are established it is no longer necessary to resolve the entire ocean basin, regional models can be employed to save computation. 
There is a significant omission of physics from this model which deserves mention here. The model is barotropic, and therefore has no baroclinic effects whatever. However, so long as the vertical structure is divisible into horizontal layers, the absolute vorticity budget still must be satisfied within each layer (as shown by the impermeability theorem of Haynes and McIntyre (1987)). This is perhaps related to the result that some quasigeostrophic baroclinic models have been observed to be inertiallydominated just as the homogeneous model is sometimes (for example, Holland and Lin (1975)). Thus, the addition of multiple layers will produce additional sources of variability (gravity waves and baroclinic instability for example) but will not affect the fundamental problem of the removal of vorticity. The question of what changes would occur were vertical structure introduced into this model is quite interesting. Even the inclusion of variations in depth in a barotropic model will have significant effects on the eddy field (as shown, for example, by Becker and Salmon (1997)).

Another perhaps significant difference between the eddy field of a barotropic model and that of a baroclinic model is the way that turbulent energy changes scale. As pointed out by Batchelor (1969) and later extended to the $\beta$-plane by Rhines (1975), the dynamics of two-dimensional (or barotropic) homogeneous turbulence are very different from that of three-dimensional (or baroclinic) homogeneous turbulence. In particular, the conservation of vorticity results in energy cascading to larger scales rather than to smaller. One could argue that inertial-domination is a natural result of this cascade to large scales. However, these results are for freely-evolving homogeneous turbulence, not forced-dissipative turbulence in the presence of a structured mean field. As shown by Smith and Vallis (2001), Smith and Vallis (2002), Smith et al. (2002), and Arbic and Flierl (2003) the forced-dissipative case is quite different from the freely-evolving case, and the presence of structures greatly changes the character of the mixing. As these calculations aren't remotely isotropic, the impact of mean flow structures is massive. Furthermore, the forcing here is at the largest scale, the basin scale, and therefore the cascade cannot proceed further in that direc- 
tion. Thus, it is difficult to make a direct connection between the results of Batchelor (1969) and Rhines (1975) and the results presented here. Indubitably, significant differences in the behavior of the eddy field would result were baroclinic effects added to the calculations here.

My approach is to address the barotropic model first, because its physics will resemble those of every layer in a layer model, although the modes of instability will be lacking. Only after this model is well-understood can the additional complexities of vertical structure be addressed. In this dissertation, it will become clear that even in the single-layer case, the physics are complex and once misunderstood. Including more layers and baroclinic instability will undoubtedly add interesting and important changes to the calculations. I expect the primary changes to be that the new mechanisms of instability are likely to produce more eddies and to produce them in different locations, and these eddies will transfer energy quite differently than barotropic eddies do. Also, later in the dissertation I speculate that profound effects may be produced if the vertical layers do not reach the boundary and end at the surface of the ocean. In this case, the absolute vorticity budget can not extend all of the way to the basin boundary. Because of the limited scope of this dissertation, the intriguing complications of vertical structure must remain the subject of future research.

\subsubsection{Definition of Eddy and Mean}

To accentuate the difference between steady-state and time-dependent calculations, I will almost always use the term eddy to refer to time-dependent motions of short time scale. Often, they will be the perturbation from a longer time-mean equilibrium. The precise meaning of the term eddy has consequences for most of the results in this dissertation.

An additional reason for this choice of definition of eddy is in comparison to data. Most of the information about the form of the wind-driven gyres is derived from observations at sea of density used to infer geostrophic velocities and surface drift. In 
both cases, synoptic observation is rarely possible. Thus, what is observed, not by a single survey, but by repeat observations of the same location is the Eulerian mean circulation. It was the observations of the Eulerian mean circulation which inspired Stommel to propose his theory of westward intensification.

Because these observations are what inspired the formulation of the steady homogeneous models used to study western intensification, the Eulerian mean is the most natural representation of the circulation in this case. The complementary definition of eddy as a perturbation from this mean follows.

In the conclusion, I have a few further remarks on the choice of the definition of eddy.

\subsubsection{Summary of the Chapters}

Chapter 2 introduces the single-gyre homogeneous model with boundary-enhanced friction in detail, and gives the primary results regarding control of the circulation strength. The primary result of the model calculations is that by increasing the viscosity in a small region very close to the boundary, the circulation strength of the entire basin can be controlled. Detailed analysis of the possible mechanisms of vorticity transport and removal in the model are analyzed in section 2.2 and diagnostics are proposed. The flux of vorticity is then analyzed in detail, and a simple test is found which determines whether the equilibrium will be western-intensified or inertially-dominated.

Because the model presented in Chapter 2 is able to be run at a much lower viscosity in the interior while remaining western-intensified, it has some extraordinary dynamics. Many of these dynamics are introduced and discussed in Chapter 3. Also, discussions of the effects of bottom friction and slip boundary are presented in Chapter 3 , because these results are helpful in understanding the results of Chapter 4 .

In Chapter 4, a second wind-driven gyre is added to consider the effects of doing so. It is demonstrated that with slip boundary conditions with two equal and opposite 
gyres, the results resemble those of Marshall (1984) with an important inter-gyre eddy flux. However, the inter-gyre vorticity flux is not as important in the case with either no-slip boundary conditions or gyres of unequal strength. Nonetheless, the circulation strength is reduced by adding the second gyre. Chapter 4 then proceeds with a review of different hypotheses of what mechanism reduces the circulation strength with the addition a second gyre. The most likely hypothesis is that the structure of the eddies which cannot form in the single-gyre but can form on the jet dividing the gyres (primarily sinuous modes) effect an efficient transport of vorticity to the boundary. 


\section{Chapter 2}

\section{Control of the Wind-Driven Single Gyre by Eddy Fluxes of Vorticity to a Region of Enhanced Viscosity}

It is unclear what controls the strength of the wind-driven ocean circulation. Traditional analytic models of the ocean circulation have relied on an interior solution in Sverdrup balance, attached to a western boundary current which is expected to close the flow of mass and remove the input of vorticity and energy from the wind. The Sverdrup interior sets the mass flux, and the western boundary current plays a passive role.

However, ultimately there must be a balance between forcing and dissipation. The wind is a constant source of energy and vorticity to the ocean. If there is not a mechanism for equilibration, it is obvious that what controls the circulation strength is unknown. This is the danger of Sverdrup's solution. It is neat, it agrees fairly well with existing observations, and it is based upon physical principles. However, since much of the physics is neglected a priori in this model, it can be only a part of a full understanding of what controls the circulation strength.

Veronis (1966b) demonstrated that as inertia becomes strong relative to dissi- 
pation $^{1}$, the inertial terms become dominant in regions outside the boundary current. More recent work has demonstrated that these inertially-dominated solutions are ubiquitous in the wind-driven single-gyre ocean model with constant viscosity: time-dependent and steady-state calculations with differing boundary conditions all demonstrate this behavior (Ierley and Sheremet (1995), Kamenkovich et al. (1995), Sheremet et al. (1995), Sheremet et al. (1997)). These inertially-dominated solutions occur at modest inertia to friction ratios, leading one to wonder what occurs in the ocean where the friction is considered to be much smaller than the inertia, yet the ocean is strongly western-intensified with inertial western boundary currents and a relatively non-inertial interior.

The resolution of the dilemma is found by examining the meaning of friction in these models. The viscosity used is not intended to represent the action of molecular viscosity; it is many orders of magnitude too large. Obviously the friction used is a parameterization of unresolved processes.

The use of a constant viscosity tacitly assumes that all the unresolved phenomena whose effects on the large scale circulation are parameterized by the friction can be treated equally. The assumption of constant viscosity in the unresolved processes is a strong and unrealistic constraint on the activity of the parameterized processes. The activity of small-scale processes in ocean observations is extremely diverse. Observations of relative dispersion of subsurface floats by LaCasce and Bower (2000) indicate approximately an order of magnitude variation in turbulent diffusivity. Additionally, the friction in simple models such as the homogeneous model represents not only the sub-grid-scale eddies, but also the interaction of the large-scale with the boundaries. It is overly optimistic to expect the effects of unresolved eddies and the effects of the interaction of the flow with topography to parameterize identically as a friction with constant viscosity.

Often, the eddy viscosity is considered as a replacement for the effects of eddies

\footnotetext{
${ }^{1}$ Veronis used bottom friction with a constant drag coefficient.
} 
only. A large Reynolds number solution was sought in these simple models because it was supposed that as the friction decreased, there would be an increase in mixing by increasingly energetic, yet well-resolved, eddies that would replace the decreased diffusion from the friction. These eddies would naturally be formed from the instabilities that occurred with increasing resolution and decreasing diffusive parametrization. More recently, the role of eddy viscosity (and eddy hyper-viscosity) has been described as merely the mechanism to set the cutoff scale of the enstrophy cascade (see, for example, Holland (1978), Marshall and Shutts (1981)), and therefore, less viscosity would only result in more small-scale enstrophy. This view neglects the important role of the frictional terms near the boundary. As demonstrated by figure 1-3, this understanding of what eddy viscosity represents is flawed. The idea that the eddies would be able to replace the friction obviously neglects the role of friction in representing interaction with the boundary by processes which will not increase with increasing resolution and increasing Reynolds number, at least not in a model without topography ${ }^{2}$.

This chapter provides an idealized framework for understanding how and where the unresolved processes in a simple barotropic model can and cannot replace the action of viscous terms.

The experiments herein attempt to verify whether the eddy viscosity can be replaced by the action of eddies in the basin interior, although not near the basin boundaries. Presumably, there are additional physical interactions near the boundaries which cannot be easily included without including additional physics (e.g., topography, tidal dissipation, separated boundary layers, etc.). The novel approach used in this chapter is to try to treat these boundary phenomena simply and see the result on the basin-wide circulation. It will be shown that while the character of the eddies changes as the viscosity is lowered in the basin interior, so long as a sufficiently strong frictional layer exists near the boundary, the mean flow is qualitatively

\footnotetext{
${ }^{2}$ Or even a model with overly smooth topography!
} 
unchanged.

In this chapter, an idealized model of a northern subtropical gyre is used which differs from the traditional model in that it has increased viscosity near the boundaries to the east and west. This is intended to represent the increased activity due to unresolved boundary physics, and there are also dynamical reasons for the choice. The primary dynamical reason, which will be derived in this chapter, is that eddies cannot remove vorticity from the basin; they merely rearrange it. In the model used here, only the friction can remove vorticity from the basin, and increasing the viscosity near the boundary is a simple representation of the unresolved physics there which should allow vorticity to be removed.

It is important to keep in mind that the enhanced viscosity is not a proposal for a viscous region near the boundaries of the ocean. It is only a very simple parameterization of boundary processes, some which might be described as viscous and some of which are certainly not, such as lee wave generation.

Of course, it is likely that a more realistic parameterization would have viscosity or other operators which are dynamically-variable as well as spatially-variable. For example, one might use a viscosity which grows with the shear of the mean flow or a quadratic bottom drag as a dynamically-dependent friction. Because most of the study will be of the time-mean circulation, where one might expect a dynamicallyvariable viscosity to be relatively stable, and because of the demands of simplicity, only spatial variations in viscosity are considered here. Furthermore, because many of these calculations tend to be strongly western-intensified, choosing a dynamicallyvariable friction may not be significantly different in gross behavior from assigning a higher value of viscosity near the western boundary. Finally, the parameterization used here makes it easy to test whether it is possible to have a western-intensified calculation with a wider inertial boundary width than frictional boundary width.

The simplicity of having only one type of friction motivates the choice of using only lateral friction with variable viscosity. Obviously, some aspects of the results 
found here will not apply when a more realistic treatment is possible. However, the boundary-enhanced viscosity acts primarily as a sink of vorticity and a generator of eddies which are properties likely to be shared by a more realistic model, it is hoped that many of the results presented here will be robust.

This chapter is organized in sections. In section 2.1, the specifics of the model used are presented. Section 2.2 presents diagnostic and analytic constructs that are used in understanding the behavior of the model. In section 2.3 , the results of the numerical calculations are presented. In the final section, the implications of these results are discussed.

\subsection{Boundary-Enhanced Viscosity in the Homoge- neous Model}

The model used here is the rigid lid, homogeneous-density, single-gyre ocean model on a $\beta$-plane with spatially-varying viscosity. Because of its relative simplicity and ease of implementation, the lateral friction used is a horizontal diffusion of relative vorticity $\left(\nabla \cdot \delta_{M}^{3} \nabla \zeta\right)$ with a spatially-varying horizontal eddy viscosity (Austausch coefficient). The viscosity is scalar and varies only in the zonal direction (i.e., perpendicular to the western boundary) in these calculations. This, together with the $\beta$-plane approximation, guarantees that the friction used is the same as if a diffusion of absolute vorticity were used (as $\nabla \cdot \delta_{M}^{3} \nabla\left(\zeta+\beta y\right.$ ) is the same as $\nabla \cdot \delta_{M}^{3} \nabla \zeta$ when $\delta_{M}^{3}$ is a function of $x$ only). Other possible choices of lateral friction operator are available such as a diffusion of momentum $\left(\left(\frac{\partial^{2}}{\partial x^{2}}-\frac{\partial^{2}}{\partial y^{2}}\right)\left[\delta_{M}^{3}\left(\frac{\partial^{2} \psi}{\partial x^{2}}-\frac{\partial^{2} \psi}{\partial y^{2}}\right)\right]+4 \frac{\partial}{\partial x} \frac{\partial}{\partial y}\left[\delta_{M}^{3} \frac{\partial}{\partial x} \frac{\partial}{\partial y} \psi\right]\right){ }^{3}$ In this section, a bottom friction with a constant and spatially-independent decay time also appears to demonstrate the role of bottom friction in the analysis, although results with bottom friction are not presented until the next chapter.

\footnotetext{
${ }^{3}$ Another, more dubious choice also not used here is $\delta_{M}^{3} \nabla^{4} \zeta$. This form is peculiar because it violates the assumption that the frictional flux should be proportional to the vorticity gradient.
} 
The model solves the following dimensionless barotropic vorticity equation (equation 2.1) and then inverts the Poisson equation (equation 2.2) to find the streamfunction at each time step:

$$
\begin{aligned}
\frac{D \zeta}{D t}+\frac{\partial \psi}{\partial x} & =-\sin (\pi y)+\nabla \cdot \delta_{M}^{3} \nabla \zeta-\delta_{S} \zeta \\
\zeta & =\nabla^{2} \psi \\
\frac{D}{D t} & \equiv \frac{\partial}{\partial t}+\delta_{I}^{2}\left(\frac{\partial \psi}{\partial x} \frac{\partial}{\partial y}-\frac{\partial \psi}{\partial y} \frac{\partial}{\partial x}\right)
\end{aligned}
$$

The dimensionless variables are related to dimensional ones in the following way (asterisks denote dimensional variables):

$$
\begin{gathered}
t_{*} \equiv \frac{t}{\beta L}, x_{*} \equiv L x, y_{*} \equiv L y, w_{E *} \equiv-W_{E} \sin (\pi y), \psi_{*} \equiv \frac{W_{E} f_{0} L}{\beta D} \psi, \\
\delta_{I}^{2} \equiv \frac{W_{E} f_{0}}{\beta^{2} D L^{2}}, \delta_{M}^{3} \equiv \frac{A_{E}}{\beta L^{3}}, \delta_{S} \equiv \frac{r}{\beta L},
\end{gathered}
$$

where $A_{H}$ is the Austausch coefficient in the horizontal direction and $r$ is the bottom friction decay rate. $L$ is the horizontal basin dimension. $D$ is the basin depth (or layer depth). The variable $\zeta$ represents relative vorticity, while $\psi$ is the streamfunction of the velocity $\left(u=-\frac{\partial \psi}{\partial y}, v=\frac{\partial \psi}{\partial x}\right)$. The streamfunction $\psi$ is scaled so that the Sverdrup solution would have a maximum of $\psi=1$ were it to fill the dimensions of the basin. The $\beta$-plane approximation is used, so the value of the Coriolis parameter at the center of the domain is $f_{0}$ and its meridional derivative is $\beta$.

The forcing is given by $w_{E}=-\sin (\pi y)$, which is a negative input of vorticity throughout the basin. This form of the wind forcing can be found in one of two ways. First, it can be found by deriving the barotropic vorticity equation from momentum equations forced by a body force proportional to $-\cos (\pi y)$, which roughly represents the tropical easterlies at $y=0$ and the westerlies at $y=1$. Second, it can be derived as the vorticity flux from an Ekman layer forced by the same wind stress whose 
pumping supplies the vorticity by vortex stretching. In either case, it is the curl of the wind stress which appears in the barotropic vorticity equation.

This single-gyre wind forcing is intended to roughly model a northern hemisphere sub-tropical gyre, so the poleward direction is the direction of increasing $y$, or north. In Chapter 4, a second gyre with vorticity input of opposite sign will be added, roughly representing a subpolar gyre. Because the goal of this chapter is to study the removal of vorticity by friction, use of a single-gyre is preferable, because there is a net input of vorticity, all of which must be removed by friction. In the double-gyre, there is no net input of vorticity, so purely internal mechanisms might control the circulation strength. I will return to this issue in Chapter 4.

The boundaries are located at $x=0$ and $x=1$ in the zonal direction and at $y=0$ and $y=1$ in the meridional direction. The boundaries are impermeable, which is implemented by setting $\psi=0$ for solutions of equation 2.2 , an appropriate method for a constant depth model. The lateral friction in equation 2.1 also requires higher-order boundary conditions. In this chapter, the eastern and western boundaries have the noslip boundary condition $\left(\frac{\partial \psi}{\partial x}=0\right)$, while the other boundaries have slip $(\zeta=0)$. For simplicity these calculations will be referred to as no-slip boundary calculations. In subsequent chapters, slip calculations will performed where the boundary conditions on all four boundaries will be slip $(\zeta=0)$.

These frictional boundary conditions were chosen because the no-slip boundary condition is good for generating instabilities at the western boundary and the slip boundary condition at the north is more easily compared to the two-gyre and doublegyre solutions presented in Chapter 4. Thus the slip boundary condition is intended to roughly represent the fluid boundaries while the no-slip boundary conditions are intended to roughly represent the solid boundaries. A recurring theme of this dissertation is that this idealization is often overly simple.

The parameters, $\delta_{I}, \delta_{M}$, and $\delta_{S}$, would be the Charney (1955), Munk (1950), and Stommel (1948) boundary layer scales, respectively, if $\delta_{M}$ were constant in a basin of 
unit width. However, in these calculations, the viscosity, and hence $\delta_{M}$, is allowed to vary. As the parameter $\delta_{M}^{3}$ is proportional to the dimensional viscosity and plays a similar role in the nondimensional equations but has different units than the viscosity, it will be called the viscosity parameter herein.

The interpretation of $\delta_{M}$ as a boundary layer width will no longer apply directly if $\delta_{M}$ varies. While the solution to the Munk problem with variable lateral viscosity (neglecting advection of relative vorticity) is not analytically tractable, the Stommel problem with a bottom drag coefficient exponentially decaying away from the coast is. In that case, the boundary layer scale changes from $\delta_{S}$ to $\delta_{d} \ln \left(\delta_{S} / \delta_{d}\right)$ for a bottom drag coefficient which is $\delta_{S} \exp \left(-x / \delta_{d}\right)$ with the boundary located where $x$ is zero.

For the calculations presented here, the value of $\delta_{I}$ is fixed at 0.02 . The value of $\delta_{M}$ varies across the different calculations and throughout the basin. The Reynolds number of the boundary layer for a given viscosity is a useful measure for comparing different runs; it is defined here as $\delta_{I}^{3} / \delta_{M}^{3}$.

When the viscosity is allowed to vary, the boundary-layer Reynolds number based on the interior viscosity $\left(\operatorname{Re}(\mathrm{int})=\delta_{I}^{3} /\left[\delta_{M}(\text { int })\right]^{3}\right)$ and the boundary-layer Reynolds number based on the viscosity at the eastern and western boundaries ( $\operatorname{Re}(b d y)=$ $\left.\delta_{I}^{3} /\left[\delta_{M}(b d y)\right]^{3}\right)$ are used as constant parameters. The viscosity parameter is

$$
\begin{aligned}
\delta_{M}^{3} & =\delta_{I}^{3}\left[\frac{1}{\operatorname{Re}(\text { int })}+\left(\frac{1}{\operatorname{Re}(\mathrm{bdy})}-\frac{1}{\operatorname{Re}(\mathrm{int})}\right)\left(e^{-x / \delta_{d}}+e^{-(1-x) / \delta_{d}}\right)\right] \\
\delta_{d} & =\frac{\delta_{I}}{\sqrt{\operatorname{Re}(\mathrm{int})}} .
\end{aligned}
$$

$\operatorname{Re}(\mathrm{bdy})$ and $\operatorname{Re}(\mathrm{int})$ are fixed. Thus, the viscosity parameter decays exponentially from the boundary value to the interior value with a decay scale equal to the frictional sublayer scale, $\delta_{d}$. This functional form was chosen for simplicity and smoothness, rather than from a rationalized parameterization of a particular physical process.

The first advantage of choosing the frictional sublayer scale as the decay scale is shown by consideration of the balance of terms in the sublayer. The frictional sublayer 
scale is the scale found by hypothesizing a balance between the mean advection and the frictional terms (see, for example, Pedlosky (1996)). This proposed balance is

$$
\delta_{I}^{2}\left(\frac{\partial \psi}{\partial x} \frac{\partial^{3} \psi}{\partial y \partial x^{2}}-\frac{\partial \psi}{\partial y} \frac{\partial^{3} \psi}{\partial x^{3}}\right)=\delta_{M}^{3} \frac{\partial^{4} \psi}{\partial x^{4}}+\frac{\partial\left(\delta_{M}^{3}\right)}{\partial x} \frac{\partial^{3} \psi}{\partial x^{3}}
$$

If the scale of the velocities is chosen to be that of the inertial boundary layer $\left(\frac{\partial \psi}{\partial x}\right.$ on the order of $\delta_{I}^{-1}$ and $\frac{\partial \psi}{\partial y}$ with a magnitude near -1$)$, then the scale over which these velocities must vary in order for this balance to be achieved is the frictional sublayer scale given above. The advantage of choosing the frictional sublayer scale as the decay scale for the viscosity parameter is that both terms on the right hand side will have the same scale. Thus, the width of the sublayer will be relatively unchanged by a change in $\operatorname{Re}(b d y)$.

The second advantage of choosing the frictional sublayer as the scale of the viscosity parameter's decay is that so long as Re(int) is greater than one, the inertial boundary layer scale will be larger than the frictional sublayer. Therefore, in all of the calculations with Re(int) greater than one, the outer part of the inertial boundary layer will not pass through the enhanced viscosity. Therefore, relatively inviscid dynamics must occur in that region.

The frictional sublayer is a theoretical construct which is useful for thinking about the way in which the frictional terms always become important near the boundaries. Even if the viscosity is extremely small, because the frictional boundary conditions must be satisfied this layer will always exist, although its scale will shrink with the viscosity. However, in a forced-dissipative problem this thin boundary layer almost always plays a critical role as there is no other region where the effects of forcing can be balanced. We shall see that this is also the case in the homogeneous single-gyre.

However, recall that the boundary-enhanced viscosity presented here is only a parameterization of boundary processes. The choice of an exponentially-decaying viscosity with a sublayer decay scale is a choice. Many of the results in this model 
will be applicable even if the boundary processes parameterized by this viscosity do not decay with this scale. This scale is chosen only to emphasize that the viscosity is being raised only within a region which was already strongly influenced by friction in this model.

Because the meridional extent of eddies is not the basin scale, their scaling is not the same as that for the mean flow. Thus, the scale of the eddies' frictional sublayer is different from that of the mean flow. It is likely that the viscosity's effect on the eddies will change differently than its effect on the mean flow changes. This is another highly desirable feature of our choice of parameterization of boundary processes.

The viscosity is chosen to vary only in $x$ for two reasons. The first is that the eastern and western boundaries are intended to be idealized solid boundaries, hence the choice of no-slip boundaries there. Thus, the unresolved but presumably enhanced boundary-related physical processes the higher viscosity is intended to represent should be present there. Second, if the viscosity varies only in $x$, then the choice between a friction term which parameterizes sub-grid-scale phenomena as a diffusion of absolute vorticity and one which diffuses relative vorticity are identical (again as $\nabla \cdot \delta_{M}^{3} \nabla(\zeta+\beta y)$ is the same as $\nabla \cdot \delta_{M}^{3} \nabla \zeta$ when $\delta_{M}^{3}$ is a function of $x$ only).

A numerical model was created to solve equations 2.1 and 2.2 with the viscosity parameter given by equation 2.3. Because of the difficulties in accurately differentiating the streamfunction four times as required to evaluate the friction term in equation 2.1, a Chebyshev pseudo-spectral method is used (see, for example, Gottlieb and Orszag (1981) and Boyd (1989)). The details of this model are presented in Appendix C.

The range of $\delta_{I}$ and $\delta_{M}$ used is governed by both numerical constraints and an attempt to reach the correct parameter range. The value of $\delta_{I}$ is 0.02 in all of the calculations presented here. The maximum interior Reynolds number with reasonable accuracy at the resolution used (256th-order polynomials) was 9 for this value of $\delta_{I}$. The accuracy at this resolution was confirmed both by spectral decay of the 
Chebyshev coefficients of relative vorticity (for all Reynolds numbers used) and by comparison with higher and lower resolution calculations (with the parameter setting: $\operatorname{Re}(b d y)=3, \operatorname{Re}(i n t)=3)$. Although the runs at different resolutions differed significantly in the details of the eddy field, the time-mean circulation and magnitude of kinetic energies agreed. Additional discussion of error can be found in section C.5.

The value of $\delta_{I}$ is physically relevant and lies in the range used in similar calculations. Ierley and Sheremet (1995) use values surrounding 0.02, Kamenkovich et al. (1995) use 0.01, and Bryan (1963) advocates a range of values from 0.03 to 0.005 depending on the depth of the moving layer and basin dimension. The value of 0.02 used here corresponds to a $200 \mathrm{~km}$ inertial boundary current scale in a $10000 \mathrm{~km}$ basin with a velocity scale of $1.6 \mathrm{~m} / \mathrm{s}$ (with $\beta=4 \cdot 10^{-11}(\mathrm{~ms})^{-1}$ ).

Although proper interpretation of eddy viscosity is model-dependent and therefore eddy viscosity is inherently difficult to measure, the value of $\delta_{M}$ used here is probably too large. The maximum value of the Reynolds number here is 9 , while estimates of a more appropriate eddy viscosity place the appropriate Reynolds number in the range of 100-1000 (Pedlosky (1987), LaCasce and Bower (2000)). Rather than increasing the Reynolds number farther in this overly-simplified model, however, it is likely more important to include additional physics. It shown below, however, that the value of $\delta_{M}$ is small enough to ensure that the frictional sublayer is clearly smaller than the inertial boundary layer, which is the desired physical criterion.

\subsection{Analysis}

In this section, some analytic results are presented which will be used throughout the dissertation. First, the vorticity equation will be written in flux form which implies that vorticity cannot be locally destroyed and therefore must be carried to the boundary to be removed. Second, the energy equation will be presented and the differences between it and the vorticity equation will be presented. Finally, budgets 
over areas of vorticity and energy will be presented. Two regions will receive particular attention, the area within a mean streamline and the basin as a whole.

The barotropic vorticity equation can be written in flux form, where every term is a conservative flux of vorticity. This is also true for the Reynolds-averaged barotropic vorticity equation.

$$
\begin{aligned}
\frac{\partial \zeta}{\partial t}+\nabla \cdot\left(\hat{\mathbf{x}} \psi+\delta_{I}^{2} \mathbf{u} \zeta-\delta_{M}^{3} \nabla \zeta+\delta_{S} \nabla \psi\right) & =-\sin (\pi y), \\
\frac{\partial \bar{\zeta}}{\partial t}+\nabla \cdot\left(\hat{\mathbf{x}} \bar{\psi}+\delta_{I}^{2} \overline{\mathbf{u}} \bar{\zeta}+\delta_{I}^{2} \overline{\mathbf{u}^{\prime} \zeta^{\prime}}-\delta_{M}^{3} \nabla \bar{\zeta}+\delta_{S} \nabla \bar{\psi}\right) & =-\sin (\pi y) .
\end{aligned}
$$

The overbar denotes a time-mean over times long compared to the eddy time scales, and primes denote fluctuations from the mean. ${ }^{4}$

Every term in equation 2.7 is a flux of vorticity except the wind-stress source. Because these terms are fluxes, the vorticity input by the wind cannot be locally removed. Actually, the wind source of vorticity can also always be written as a flux rather than a source, as it is the curl of the wind stress itself. The time-derivative of vorticity can also be written as a flux if helpful. The result that all of the terms in the equation are fluxes is the impermeability theorem for this system (Haynes and McIntyre (1987), Marshall and Nurser (1992)). For the single-gyre, the wind's vorticity input is negative everywhere. Therefore, no amount of internal mixing of the vorticity can cause internal cancellation; the vorticity must be fluxed from the input region out of the basin. As is demonstrated in equation 2.7 , the flux of vorticity can be carried by the $\beta$-term, the mean advection of mean relative vorticity, the eddy advection of eddy relative vorticity, bottom frictional flux, or by a lateral frictional flux. In this dissertation, these terms will be called the $\beta$-flux, the mean flux, the Reynolds flux, and the frictional flux of vorticity respectively. The fluxes that will be

\footnotetext{
${ }^{4}$ This form of the equations is certainly valid when the value of $\delta_{S}$ is constant. However, just as using a lateral friction which diffuses momentum rather than vorticity yields a different form for the lateral friction operator, the form of the bottom friction will vary depending on whether it is intended to be a bottom drag on momentum, a flux of vorticity, an Ekman layer on the ocean bottom, etc.
} 
most often discussed in the Reynolds-averaged equation are:

$$
\begin{aligned}
\beta-f l u x & \equiv \psi \hat{\mathbf{x}}, \\
\text { mean }-f l u x & \equiv \delta_{I}^{2} \overline{\mathbf{u}} \bar{\zeta} \\
\text { eddy }-f l u x & \equiv \delta_{I}^{2} \overline{\mathbf{u}^{\prime} \zeta^{\prime}} \\
\text { bottom }- \text { flux } & \equiv \delta_{S} \nabla \bar{\psi}, \\
\text { lateral }- \text { flux } & \equiv-\delta_{M}^{3} \nabla \bar{\zeta} .
\end{aligned}
$$

Unlike vorticity, the energy can be locally dissipated. The equation for the kinetic energy of the mean flow $\left(\bar{E} \equiv \bar{\psi}^{2} / 2\right)$ can be constructed from the vorticity equation 2.6 above by multiplication by $-\psi$ and use of product rules for differentiation.

$$
\begin{aligned}
& \frac{\partial E}{\partial t}+\nabla \cdot\left[-\psi \frac{D}{D t} \nabla \psi+\delta_{I}^{2} \mathbf{u} E-\frac{\psi^{2}}{2} \hat{\mathbf{x}}+\psi \delta_{M}^{3} \nabla \zeta-\delta_{S} \nabla \frac{\psi^{2}}{2}-\delta_{S} \psi \mathbf{u}\right] \\
= & \delta_{M}^{3}\left[\nabla^{2} E-\left(\frac{\partial^{2} \psi}{\partial x^{2}}\right)^{2}-\left(\frac{\partial^{2} \psi}{\partial y^{2}}\right)^{2}-2\left(\frac{\partial^{2} \psi}{\partial x \partial y}\right)^{2}\right]-2 \delta_{S} E+\psi \sin (\pi y) .
\end{aligned}
$$

For the Reynolds-averaged version, multiply equation 2.7 by $-\bar{\psi}$, producing

$$
\begin{gathered}
\frac{\partial \bar{E}}{\partial t}+\nabla \cdot\left[-\bar{\psi} \frac{\bar{D}}{D t} \nabla \bar{\psi}+\delta_{I}^{2} \overline{\mathbf{u}} \bar{E}-\frac{\bar{\psi}^{2}}{2} \hat{\mathbf{x}}+\bar{\psi} \delta_{M}^{3} \nabla \bar{\zeta}-\delta_{S} \nabla \frac{\bar{\psi}^{2}}{2}-\delta_{S} \bar{\psi} \overline{\mathbf{u}}\right] \\
+\nabla \cdot\left[\delta_{I}^{2} \overline{\mathbf{u}} \cdot \overline{\mathbf{u}^{\prime} \mathbf{u}^{\prime}}-\bar{\psi} \delta_{I}^{2} \overline{\mathbf{u}^{\prime} \cdot \nabla \nabla \psi^{\prime}}\right] \\
=\delta_{M}^{3}\left[\nabla^{2} \bar{E}-\left(\frac{\partial^{2} \bar{\psi}}{\partial x^{2}}\right)^{2}-\left(\frac{\partial^{2} \bar{\psi}}{\partial y^{2}}\right)^{2}-2\left(\frac{\partial^{2} \bar{\psi}}{\partial x \partial y}\right)^{2}\right]-2 \delta_{S} \bar{E}+\bar{\psi} \sin (\pi y) \\
+\delta_{I}^{2}\left(\overline{\mathbf{u}^{\prime} \mathbf{u}^{\prime}} \cdot \nabla\right) \cdot \overline{\mathbf{u}} .
\end{gathered}
$$

The terms after the equal sign in this equation are local sinks and sources of energy.

All of the frictional terms in equation 2.14 are negative definite except for $\delta_{M}^{3} \nabla^{2} E$ which represents a smoothing of sharp energy gradients. The equivalent term does not appear as a source term in the energy equation if frictional diffusion of momentum rather than diffusion of relative vorticity is used. In that case, this term is a flux of 
energy rather than a sink. However, in practice, this term is usually small when compared to the negative definite terms, and the sum of the frictional terms is always negative on integration over the basin (although it is sometimes locally a small positive quantity).

The wind energy input term can be of either sign, and the amount of energy depends on the solution through multiplication by $\psi$ as the work done differs depending on the solution's alignment with the wind stress. Because of the variable nature of the energy source and the existence of local sources and sinks in the energy equation, the vorticity budget is a simpler tool for analysis of this model. Further discussion of the energetics of the model is presented in section 3.6.

The integral of the vorticity equation within a mean streamline is useful in understanding the role of eddy and frictional fluxes in this model. If the mean is taken over the entire time interval, then $\frac{\partial \bar{\zeta}}{\partial t}$ vanishes. Using the divergence theorem and noting that the mean fluxes cancel out when integrating along a mean streamline, the following results for the area enclosed by the streamline where $\psi=\psi_{C}$ :

$$
\oint_{\psi_{c}}\left(\overline{\mathbf{u}^{\prime} \zeta^{\prime}}+\delta_{S} \nabla \bar{\psi}-\delta_{M}^{3} \nabla \bar{\zeta}\right) \cdot \hat{\mathbf{n}} d s=-\iint_{\psi \leq \psi_{c}} \sin (\pi y) d^{2} A
$$

Thus, the flux across mean streamlines can only be carried by the friction or by the eddies.

If the streamline chosen is the one located at the boundary $\left(\psi_{C}=0\right)$, the basinwide budget is produced.

$$
\oint_{\psi_{c}}\left(\delta_{S} \nabla \bar{\psi}-\delta_{M}^{3} \nabla \bar{\zeta}\right) \cdot \hat{\mathbf{n}} d s=-\frac{2}{\pi}
$$

It is obvious from equation 2.16 that it is exceedingly difficult to reduce the viscosity at the boundary without affecting the mean flow.

Equation 2.16 is the reason for this dissertation's emphasis on unresolved bound- 
ary processes. Regardless of the model used, because the vorticity equation can be written in flux form, there must always be a process at the edges of the domain capable of removing the vorticity. The model chosen here is enhanced viscosity at the boundary, which obviously relieves the strict nature of equation 2.16 which is evaluated using the value of the viscosity at the boundary only. By using a large viscosity at the boundary, equation 2.16 can be satisfied and by simultaneously using a small interior viscosity equation 2.15 can be dominated by resolved eddies rather than by the parameterization.

If the boundary condition is no-slip, then $\delta_{S} \nabla \bar{\psi}=0$, and neither bottom friction nor eddies can contribute to the basin-wide vorticity budget. For this reason, the bottom friction is not used for most of the calculations presented in this chapter $\left(\delta_{S}=\right.$ 0 ). Calculations with bottom friction are presented in the next chapter and reveal that adding bottom friction produced qualitatively different equilibrium solutions only when the bottom friction was strong enough to strongly reduce the presence of eddies.

The mean flow energy budget for the entire basin is obtained by integrating the energy equation.

$$
\begin{aligned}
0 & =\iint\left(\delta_{M}^{3}\left[\nabla^{2} \bar{E}-\left(\frac{\partial^{2} \bar{\psi}}{\partial x^{2}}\right)^{2}-\left(\frac{\partial^{2} \bar{\psi}}{\partial y^{2}}\right)^{2}-2\left(\frac{\partial^{2} \bar{\psi}}{\partial x \partial y}\right)^{2}\right]\right) d^{2} A \\
& +\iint\left(-2 \delta_{S} \bar{E}+\delta_{I}^{2}\left(\overline{\mathbf{u}^{\prime} \mathbf{u}^{\prime}} \cdot \nabla\right) \cdot \overline{\mathbf{u}}+\bar{\psi} \sin (\pi y)\right) d^{2} A
\end{aligned}
$$

The energy in the mean flow can be removed by lateral or bottom friction or by turbulent kinetic energy production. Unlike the vorticity budget, the energy budget does not obviously benefit from enhancing the viscosity in any particular region.

\subsubsection{Interpreting Eddy Flux Divergences}

In many of the figures in this dissertation, the emphasis is placed on the eddy flux divergences, not the fluxes themselves. This is because the fluxes are not easily 
determined at a particular location; the interpolation and statistical problems are challenging. Integrals of the flux divergence, however, have better statistical properties as they are effectively averages over a large area, and it is much easier to do proper interpolation for fluxes out of areas of integration than to try to locate a particular streamline and interpolate the fluxes across it. Also, much of the information contained in the flux field is not relevant to understanding the effects of the eddies on the mean flow.

Only the divergences of the fluxes appear in the vorticity equation 2.6, and this is also true of the vorticity equation for the mean field (equation 2.7). Therefore, in determining the effects of the eddies on the mean flow, it is more important to determine the flux divergences than the fluxes themselves.

However, it is often more physically intuitive to consider fluxes, or at least to connect the divergences to hypothetical fluxes which might be responsible for them. Figure 2-1 schematizes two methods which I found useful for converting between the fluxes and the flux divergences. These diagrams represent regions of integration of the flux divergence field. Upon integration over a region, the flux divergence is converted to an integral of the total flux out of that region. With a little practice, the result of these integrals becomes intuitive, and the flux divergence field can be more readily interpreted.

Figure 2-1a schematizes integration of the flux divergence field within time-mean streamlines of the flow, as imagined in equation 2.15. If the mean streamfunction is relatively simple topologically, as are most of the single-gyre calculations then there is a region where the time-mean streamfunction is dominantly positive, and possibly a region where the streamfunction is negative. Within the positive region, the streamlines will largely be nested one within another, and likewise for the negative streamlines.

Thus, it is sensible to integrate over the entire region where $\bar{\psi}$ exceeds a certain value when the streamfunction is positive. The arrows indicating the total flux into 
the streamline located in the upper, unshaded portion of figure 2-1a, which is obtained upon integrating the flux divergence within that streamline. If the streamlines are neatly nested inside that streamline, integrating within that streamline amounts to integration over the whole region where $\bar{\psi}$ exceeds its value on this streamline. As equation 2.15 demonstrates, in this model only eddy fluxes and frictional fluxes can have a total flux across a mean streamline. The arrows in this schematic are pointing inward because typically these fluxes converge to deposit positive vorticity in the region enclosed by a closed streamline which cancels the negative vorticity input by the wind. Equivalently, the fluxes can be thought of as outward fluxes of the negative vorticity input by the wind, because the flux of negative vorticity tends to diverge within the streamline. Therefore, by analyzing these fluxes, we can determine how much of the wind input within this streamline is carried out by the eddies and how much is carried out by the friction.

Likewise, we can integrate over all regions where the streamfunction is less than a particular value. This is particularly useful for negative values of the streamfunction. The arrows into of the shaded region enclosed by a mean streamline in the lower part of figure 2-1a schematize such an integration.

Note that the arrows are pointing inward here as well. There is a choice of convention implied here which is used in this dissertation. The sign of the fluxes are interpreted as toward the boundary (that is, out of a closed streamline, not in), regardless of the sign of the streamfunction. Thus, these fluxes are inward, so they can balance the negative wind input within the mean streamlines. This convention treats the negative and positive streamlines most simply, because it is easy to imagine a budget of the wind input within a closed streamline being balanced by the frictional and eddy fluxes across the streamline.

However, when the topology of the streamfunction is not simple some complications arise. The primary example of this is the $\bar{\psi}=0$ streamline. Because of the impermeability boundary condition, this streamline surrounds the entire basin, but it 
also divides the regions of positive and negative streamfunction. We can generate at least three sensible calculations of the total flux across the $\bar{\psi}=0$ streamline. First, we could integrate over the whole basin. This would give the flux out of the basin, and it is useful for calculating the total vorticity budget of frictional removal and wind input described in equation 2.16. Second, if we calculate the flux by integrating the divergence over all regions whose streamfunction is greater than zero, a different result will be obtained. In this case, it would be the total flux out of all of the unshaded regions in figure 2-1a. For some of the streamline bounding this region this flux would be through the boundary, but in other areas it is into the shaded regions of negative streamfunction. Third, we could calculate the total flux out of the shaded regions. Some of this flux would be through the boundary and some would be into the unshaded regions. It should be clear that by cancellation along the shared boundaries, the sum of the flux out of the shaded region and the flux out of the unshaded region will sum to be the flux out of the basin. Also, if the flux vanishes at the basin boundary as the eddy flux does, then each flux will have the same magnitude, that of the flux from the shaded to the unshaded regions. This result is useful in section 3.3 .

A lesser problem exists when there are shaded regions separated from each other. Then, it will not be the flux out of a particular closed streamline which is calculated by integrating over the region where $\bar{\psi}$ is less than a particular value. Because of the topology of the time-mean streamfunctions in this chapter and the next, this is usually not a big problem, and typically these separated areas will be clumped together to yield the flux out of the combined region.

Thus, the convention used in the figures displaying the flux across mean streamlines is as follows. For all regions where the mean streamfunction is positive, the fluxes are calculated by integrating the divergence over the region where $\bar{\psi}$ exceeds a certain value. Where the streamfunction is negative, the fluxes are calculated by integrating the divergence over the region where $\bar{\psi}$ is less than a certain value. At the zero streamline these fluxes will not agree as they are fluxes across different closed 


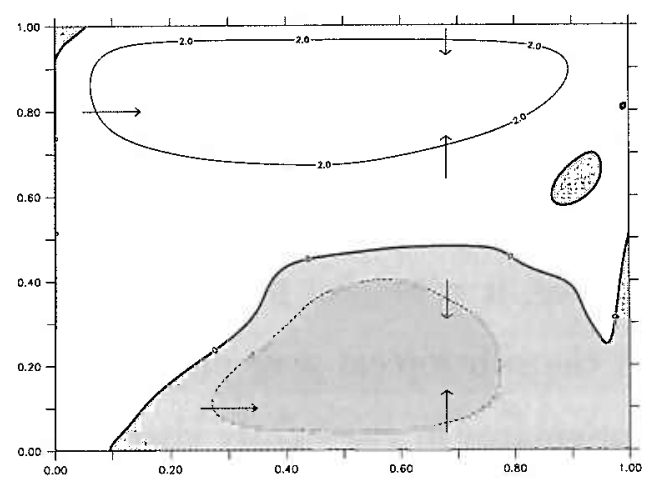

a.

Figure 2-1: Schematics of regions of integration useful in converting from flux divergences to flux out of a particular region. (a) shows the fluxes out of mean streamlines (regions of negative streamfunction are shaded), and (b) shows the fluxes out of a box connected to the boundaries. The eddy flux convergence $\left(-\nabla \cdot \overline{u^{\prime} \zeta^{\prime}}\right)$ is shaded beneath.

loops of the $\bar{\psi}=0$ streamline, but their sum will be the flux out of the basin. From equation 2.16, we expect that the sum of the eddy fluxes will be zero while the sum of the frictional fluxes will be the wind input.

Figure 2-1b schematizes a different conversion from flux divergences to fluxes. The eddy flux convergences are shaded in this figure. If the flux convergences are integrated within this box, the total flux across the box's boundary inward will be obtained. As the eddy fluxes vanish at the boundaries, only the fluxes across the interior portion of the box where the arrows are need be considered. Frictional fluxes do not automatically vanish at the boundary, so they must be analyzed point by point along the boundary to determine how much of the flux remains in the basin and how much is transported out. In the figure, a small region of eddy vorticity flux convergence (light coloring) is inside the box. The convergence acts against the wind input, that is, the negative vorticity input of the wind is transferred to an outward eddy flux of negative vorticity there. Equivalently, a positive eddy vorticity flux into the box results, which is indicated by the arrows. If the box were extended just 
slightly more west and north, at $x=0.1$ and $y=0.9$, then a region of eddy divergence would be included. In this case, the eddy vorticity flux would be, on average, out of the box.

Continuing in this manner, the time-mean transport of vorticity by the eddy field can be studied. Since the wind input is negative, it is helpful to think of the eddy fluxes transporting the wind input, which in the subtropical gyre provides negative vorticity. These fluxes' vorticity transport originates in the lightly shaded regions, where they draw off negative vorticity from the mean flow and they transport it to the darkly shaded regions, where they add negative vorticity to the mean flow. Note that the reason for considering the convergences is that the path which the eddy fluxes take may be circuitous or obscured by fluxes which do not interact locally with the mean flow, as the curl of the eddy fluxes is typically quite large. Only the divergent eddy fluxes interact with the mean flow, so directly examining the circuitous paths of the eddy fluxes can be misleading. However, the direct examination of the fluxes is rarely necessary since the eddy fluxes vanish at the basin boundary, so eventually the vorticity transported from a divergent region by eddy fluxes must end up being deposited in a convergent region. A useful analogy to the eddy fluxes of vorticity is the transport of a passenger-carrying train. To determine which stations will change in population and where crowds will form, one need only know where the train picks up and drops off passengers, not how many are on the train.

It can be difficult to determine exactly which divergent region is the source of a flux to a particular convergent region when there is more than one region of convergence and divergence. In this case a more careful study needs to be made. Often all of the fluxes-eddy, beta, mean, and frictional-must be considered. Study of integration and budgeting over different regions is required to decide where the fluxes originate and end. I generally have left the details of this analysis out of the discussion and mention the final results of the calculations in the text. 


\subsection{Computational Results}

The major computational results found with this model are presented here as subsections. The first subsection shows that increasing the viscosity only within the frictional sublayer controls the overall circulation. In the second subsection, it is revealed that outside the frictional sublayer, the eddy flux of vorticity is responsible for decreasing the mean circulation.

\subsubsection{Control of Circulation Strength}

Figure 2-2 shows the time-mean streamfunction ${ }^{5}$ resulting from different values of $\operatorname{Re}(\mathrm{bdy})$ and $\operatorname{Re}(\mathrm{int})$. For a given $\operatorname{Re}(\mathrm{int})$, the circulation strength can be reduced by decreasing $\operatorname{Re}(\mathrm{bdy})$. As shown in the introduction, this is not true of a steadystate calculation, because much of the mean flow never passes through the region of enhanced viscosity. Therefore, when eddies are present, the circulation in the interior is controlled non-locally by the frictional sublayer at the boundary.

The mean flow is comparable between some of the calculations and those with higher $\operatorname{Re}(\mathrm{int})$ but lower $\operatorname{Re}(\mathrm{bdy})$, e.g., the mean streamlines of the $\operatorname{Re}(\mathrm{bdy})=0.5$, $\operatorname{Re}($ int $)=3$ calculation and the $\operatorname{Re}($ bdy $)=0.25, \operatorname{Re}($ int $)=5$ calculation are surprisingly similar. Likewise the $\operatorname{Re}(\mathrm{bdy})=3, \operatorname{Re}($ int $)=3$ time-mean resembles the $\operatorname{Re}(\mathrm{bdy})=0.25$, $\operatorname{Re}($ int $)=8$ calculation. When two solutions have the similar time-mean streamfunctions, I will call them homoparic, for same mean. Section 3.2 will deal with homoparic solutions in detail.

Curiously, some regions of the less viscous calculations are shaded. This means that their mean flow rotates in a direction counter to the direction of the wind forcing. These counter-rotating regions will be discussed further in section 3.3.

Figure 2-3 compares snapshots of the vorticity and streamfunction from a homoparic pair. The lowered value of $\operatorname{Re}(\mathrm{int})$ is apparent in the wispy vorticity field

\footnotetext{
${ }^{5}$ The time-means are taken over the second half of the total integration time here and in most of the dissertation. Exceptions to this rule are mentioned in the text.
} 


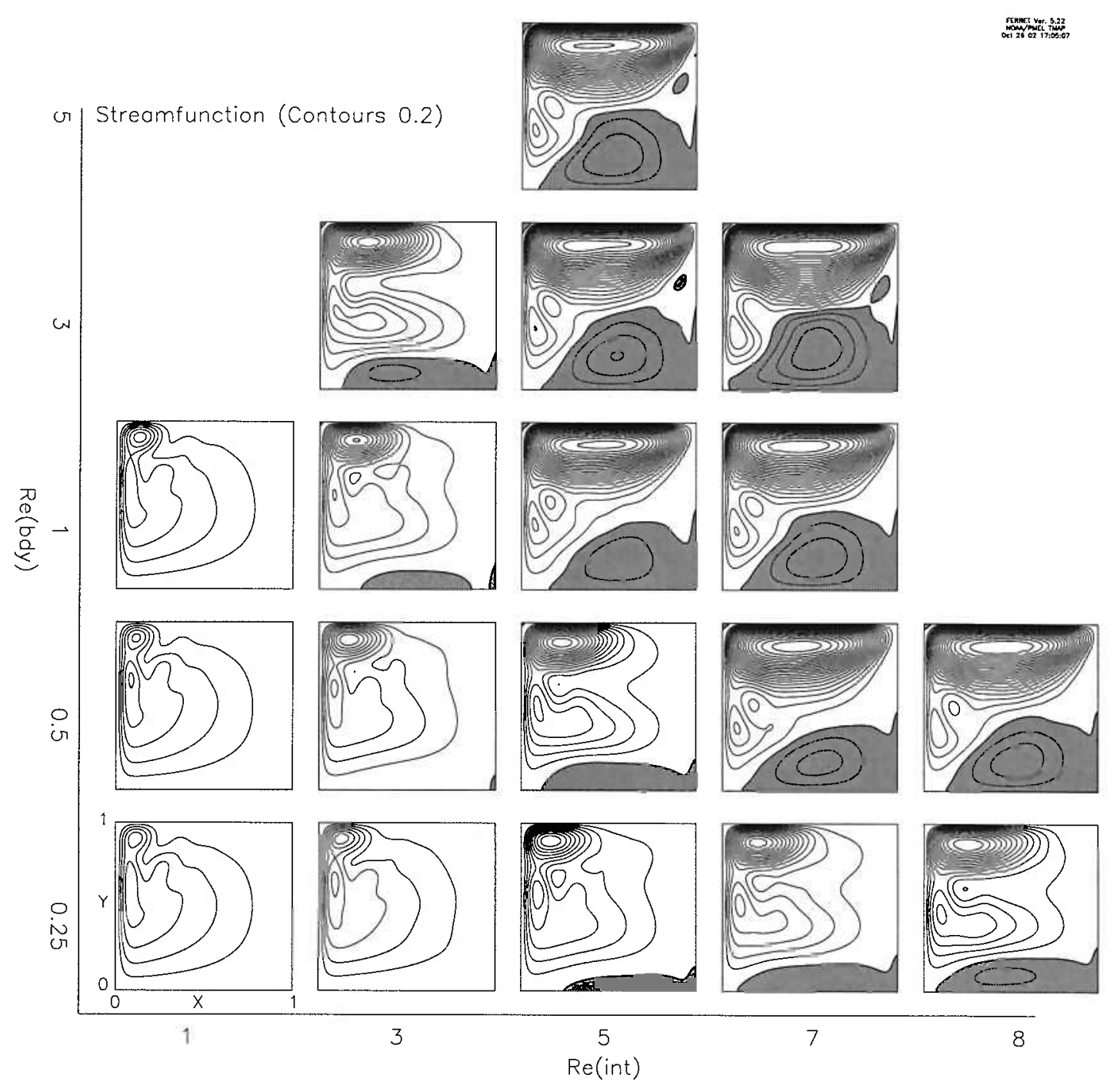

Figure 2-2: Collage of contours of the time-mean streamfunction for different values of $\operatorname{Re}(\mathrm{int})$ and $\operatorname{Re}(\mathrm{bdy})$. The contour interval is 0.2 in units where 1 is the maximum of the Sverdrup solution. Regions of negative streamfunction are shaded. 


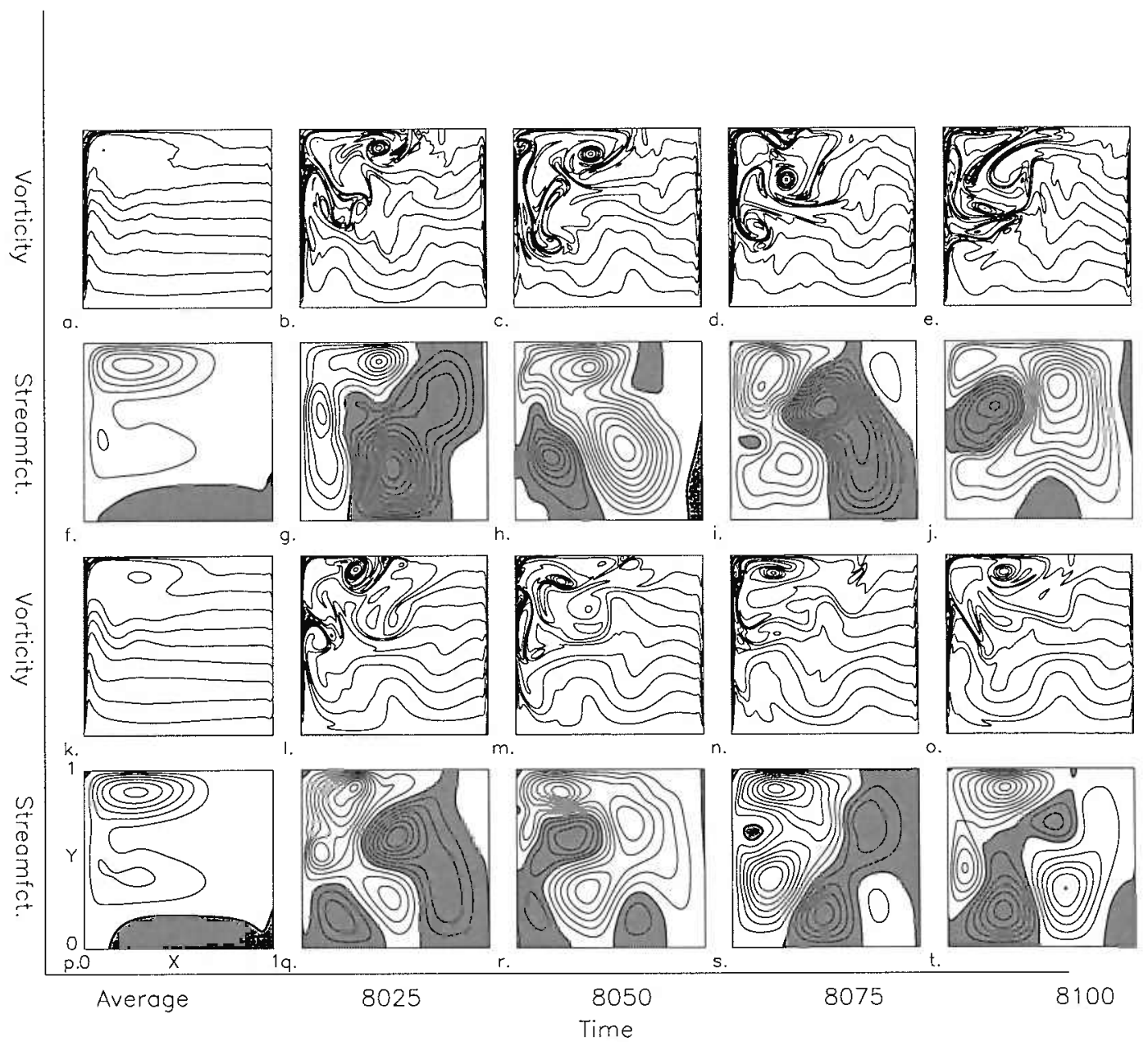

Figure 2-3: Snapshots and the time-mean of absolute vorticity and streamfunction are given for the $\operatorname{Re}(b d y)=0.25, \operatorname{Re}($ int $)=8$ and $\operatorname{Re}(b d y)=3, \operatorname{Re}($ int $)=3$ calculations. The time-means are in $(\mathrm{a}),(\mathrm{f}),(\mathrm{k})$, and $(\mathrm{p})$. The upper two rows show the $\operatorname{Re}(\mathrm{bdy})=0.25$, $\operatorname{Re}($ int $)=8$ calculation. The lower two rows show the $\operatorname{Re}(b d y)=3, \operatorname{Re}($ int $)=3$ calculation. Contours are 0.1 for vorticity and 0.5 for streamfunction. Regions of negative value are shaded.

around the eddies. Closer analysis reveals that the mean flow vorticity dynamics for these homoparic calculations differ only slightly, yet the eddies are quite different. More detailed analysis is presented in section 3.2. 

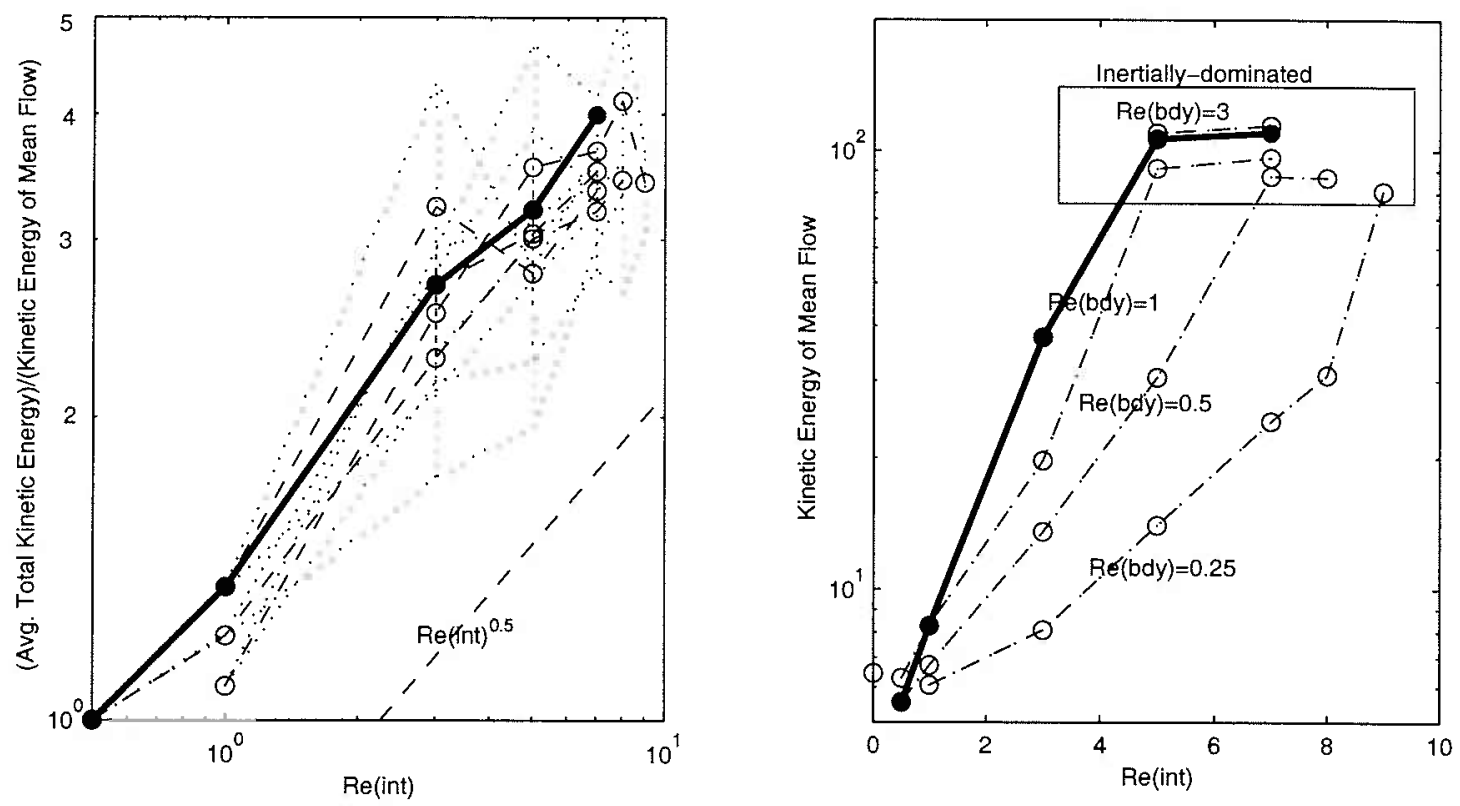

Figure 2-4: (a) plots the ratio of the time-mean of the kinetic energy $\left(\int \overline{\psi^{2}} \mathrm{~d}^{2} \mathrm{~A} / 2\right)$ to the kinetic energy of the time-mean flow $\left(\int \bar{\psi}^{2} \mathrm{~d}^{2} \mathrm{~A} / 2\right)$ for calculations with different $\operatorname{Re}($ int $)$ and $\operatorname{Re}(b d y)$. The dotted lines show the range of variability during the interval of averaging. A line proportional to Re(int $)^{1 / 2}$ is included for comparison, because the ratio scales approximately as $\operatorname{Re}(\mathrm{int})^{1 / 2}$. The ratio is relatively insensitive to the value of $\operatorname{Re}(\mathrm{bdy})$, so the energy in the eddies is strongly affected by $\operatorname{Re}($ int $)$ but not by $\operatorname{Re}\left(\right.$ bdy). (b) shows the kinetic energy of the time-mean flow $\left(\iint \bar{\psi}^{2} \mathrm{~d}^{2} \mathrm{~A} / 2\right)$ from calculations with different $\operatorname{Re}(\mathrm{bdy})$ and $\operatorname{Re}(\mathrm{int})$. In both (a) and (b), the dashed lines connect the values for a particular value of $\operatorname{Re}(\mathrm{bdy})$, and the thick line connects the values for constant viscosity parameter, i.e., $\operatorname{Re}(b d y)=\operatorname{Re}($ int $)$.

The relationship between the kinetic energy of the mean flow and the total kinetic energy (which includes both eddy and mean flow energy) changes as the viscosity decreases. Figure 2-4b shows that the kinetic energy contained in the mean flow is reduced by decreasing $\operatorname{Re}(\mathrm{bdy})$. Figure 2-4a shows that the total kinetic energy continues to increase with increasing $\operatorname{Re}(\mathrm{int})$ despite changes to $\operatorname{Re}(\mathrm{bdy})$. Thus, the control of the mean flow also extends to the energy contained in the mean flow, because as $\operatorname{Re}($ int $)$ increases a larger proportion of the kinetic energy resides in the eddy field rather than the mean flow. 


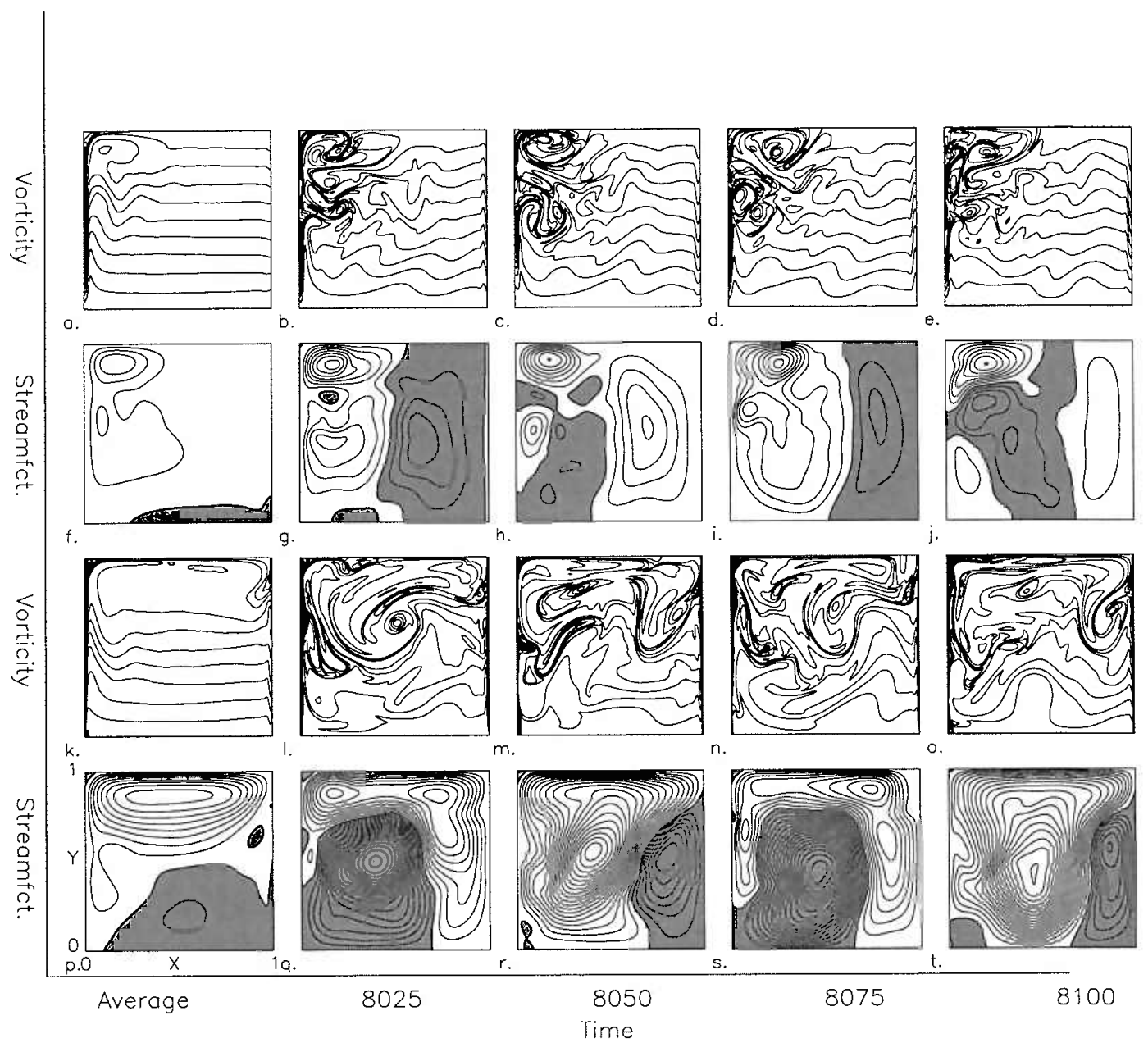

Figure 2-5: Snapshots and the time-mean of absolute vorticity and streamfunction are given for the $\operatorname{Re}(\mathrm{bdy})=0.25, \operatorname{Re}(\mathrm{int})=5$ and $\operatorname{Re}(\mathrm{bdy})=5, \operatorname{Re}(\mathrm{int})=5$ calculations. The time-means are in (a),(f),(k), and (p). The upper two rows show the $\operatorname{Re}(\mathrm{bdy})=0.25$, $\operatorname{Re}($ int $)=5$ calculation. The lower two rows show the $\operatorname{Re}($ bdy $)=5, \operatorname{Re}($ int $)=5$ calculation. Contours are 0.1 for vorticity and 0.5 for streamfunction. Regions of negative value are shaded. 
Figure 2-5 shows typical snapshots and time-means of the absolute vorticity and streamfunction for an inertially-dominated and a western-intensified calculation with the same Re(int). The eddy activity is strongest in the west for the western-intensified solution. In both cases, the eddy activity interacts with the recirculation gyre.

\subsubsection{Mechanism of Vorticity Transport}

As discussed in the introduction, the transport of vorticity is critical to the control of the circulation strength. Thus, the transport of vorticity in each calculation has been diagnosed. Ultimately the friction must remove the vorticity from the basin but en route there are many possible pathways for transport of vorticity. In all but the most viscous of these calculations, the eddy fluxes dominate the transport of vorticity across mean streamlines in the basin interior, although they are not often the dominant term in the vorticity equation. Only in a thin layer near the boundary does the transport across mean streamlines become frictional.

In this section we will first diagnose the eddy and frictional flux divergences to see the effects of these processes on the mean flow. The flux divergences of westernintensified and inertially-dominated solutions will be contrasted, as well as the flux divergences of the members of a homoparic pair. Then the fluxes themselves will be analyzed. Finally, the effects of raising and lowering the viscosity on the meridional averages of the terms in the vorticity equation will be demonstrated, and they will reveal a simple predictor of whether a calculation will have an equilibrium which is inertially-dominated or western-intensified.

Figure 2-6a shows the action of the frictional and eddy fluxes of vorticity on the mean flow for a western-intensified solution. ${ }^{6}$ The contours of the mean flow and the eddy flux convergence are shown. The eddies interact with the mean flow in two primary regions, within the western boundary current and within the recirculation gyre.

\footnotetext{
${ }^{6}$ The reader may wish to review section 2.2 .1 to help interpret these flux convergences.
} 

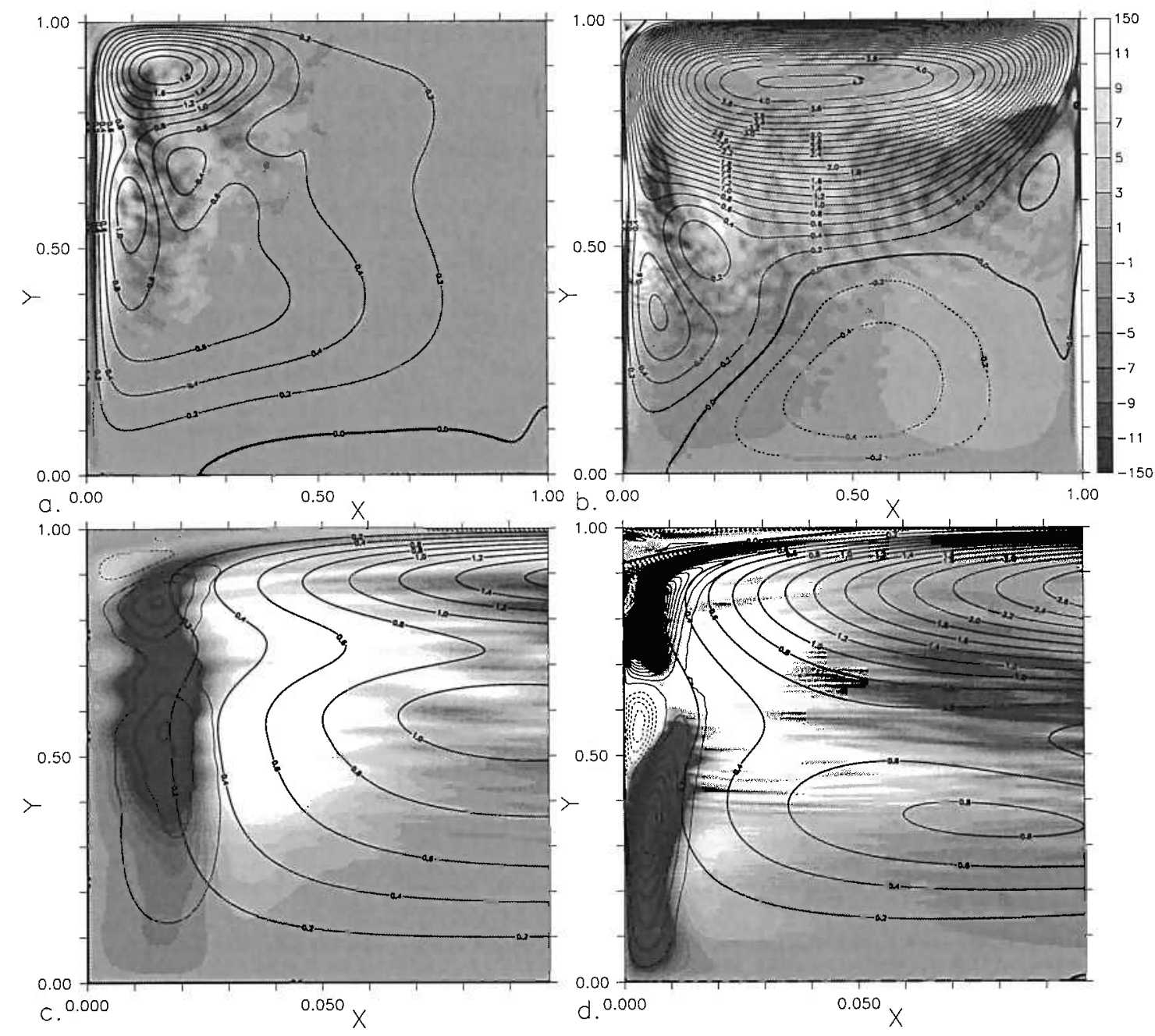

Figure 2-6: (a) The time-average streamfunction contours are superimposed on the eddy flux convergence (shaded) for the $\operatorname{Re}(\mathrm{bdy})=0.25, \operatorname{Re}(\mathrm{int})=5$ calculation. Lighter shading denotes convergences of $\overline{\mathbf{u}^{\prime} \zeta^{\prime}}$ (which remove vorticity of the sign of the wind input from the mean flow); darker shading denotes divergences. (b) is similar to (a) but for $\operatorname{Re}(\mathrm{bdy})=5, \operatorname{Re}(\mathrm{int})=5$. (c) and (d) are close-ups of (a) and (b), respectively, near the western boundary. In (c-d), the friction flux divergence is also superimposed with thin lines (contours $=10$, no zero contour, dashed/solid for divergence/convergence). 
Figure $2-6 c$ is a close-up of figure 2-6a, and it shows that within the western boundary current the effect of the eddies is to transport the wind's vorticity input in the interior of the basin toward the western boundary. Once there, the frictional dissipation disposes of it. ${ }^{7}$ The white region of eddy flux convergence at approximately $x=0.05$ removes negative vorticity (of the sign of the wind input) from the mean flow, and the black region of eddy flux divergence near $x=0.02$ deposits this negative vorticity closer to the boundary. The frictional contours above this black region in figure 2-6c indicate that the frictional dissipation receives this negative vorticity from the eddies. Because there are few areas of dotted friction contours, we see that the friction is able to remove negative vorticity from the basin, as anticipated by equation 2.16. In the western boundary current, the eddies' effect is to widen the influence of the frictional dissipation toward the basin interior. The effect of eddies here is reminiscent of the effect of eddies in traditional, non-rotating turbulent boundary layers.

Within the recirculation gyre, the eddies are active while the frictional terms are less so. In this region the eddies transport the majority of the vorticity input across the mean streamlines (see equation 2.15). This will be quantified later. The second effect of the eddies in the recirculation gyre is to homogenize the absolute vorticity (as theorized by Rhines and Young (1984). As can be seen in figure 2-5a and figure 2-5k, the time-mean absolute vorticity is smoothed within the recirculation gyre.

In figure 2-6b and 2-6d, the flux convergences and mean flow are shown for a calculation which is inertially-dominated. Note the eddy activity generated at the eastern boundary near $y=0.85$ in figure $2-6 \mathrm{~b}$. This activity is important in regulating the strength of the inertially-dominated solutions. The importance is made clear by noting that in figure 2-4b that the inertially-dominated solutions have qualitatively different parametric dependence on Reynolds number than the western-intensified solutions. When the western frictional sublayer removes too little of the wind's vorticity

\footnotetext{
${ }^{7}$ Equivalently, one can consider the eddies to flux positive vorticity from the western boundary to the basin interior where it cancels the negative vorticity input from the wind.
} 
input, the recirculation continues to build up negative vorticity and enlarges until the recirculation reaches the eastern boundary. Once the eastern boundary is reached, the eddy activity produced there changes the response of the circulation because the eddies are able to tear vorticity from both the eastern and western boundaries' frictional sublayers. Because of the qualitative change in behavior when the recirculation reaches the eastern boundary, reaching it is the criterion used herein to distinguish western-intensified from inertially-dominated solutions. In section 3.4, it is shown that a qualitative change in the temporal behavior of the solutions also occurs when the recirculation gyre reaches the eastern boundary.

One might wonder why the eastern boundary is needed instead of the northern boundary. In fact, due to a small trapped eddy that forms in the northwest corner where the western boundary current turns to the east, which apparently is present only with no-slip conditions, the net effect of the frictional flux through the northern boundary is to add to the wind input of vorticity by a small amount ( 0.64 wind input, $O(0.05)$ through the northern boundary, depending on the Reynolds number). Similar phenomena have been found in the corners in analytic asymptotic treatments of other high Reynolds number fluid problems (some are mentioned, for example in Cowley (2001)). The northern boundary is therefore not effective in helping to remove vorticity from the basin in these calculations at high Reynolds number.

There is a white region of eddy flux convergence within the frictional sublayer of the western boundary current ( $x$ near $0, y$ between 0.5 and 0.7 ) in figure 2-6d that is not present in figure figure 2-6c. This region of convergence is consistent with the notion that the viscosity is insufficient in figure $2-6 \mathrm{~d}$ to remove all of the vorticity brought into the frictional sublayer by the mean flow and eddies, because this white region indicates that the eddy fluxes' supply of the wind's vorticity input to the frictional sublayer reverses direction to transport negative vorticity out of the white region. In contrast, the black regions in the frictional sublayer figures 2-6c-d import negative vorticity from eddy fluxes to be removed from the basin by friction. 
The black regions can equivalently be considered as sources of positive vorticity brought into the basin by friction. This positive vorticity is transferred by the eddy flux to white regions where it is used to cancel the wind input of negative vorticity. The wind's vorticity input is in turn brought to the white regions primarily by the mean flow. In the white region of the frictional sublayer figure 2-6d, negative vorticity is brought into the basin by friction and fluxed by eddies to black regions where it adds to the wind input of negative vorticity. The amount of vorticity brought into the basin must equal the wind input for an equilibrium to be reached, but if the frictional flux changes sign in this white region, this means that the frictional flux elsewhere must be larger for a basin-wide equilibrium to be reached. Thus, the solution must increase the relative vorticity gradients near the boundary. The increase in these gradients changes the dynamics to be less and less like those of the linear Munk solution or those at lower Reynolds number.

It will be shown below that the net result of this white convergent region on the eddy dynamics is that the eddy flux to the frictional sublayer becomes no more effective than the mean flux in delivering negative vorticity to the western frictional sublayer. Thus, the eddy's advantage over the mean flow, that it can flux vorticity across mean streamlines, is wasted.

The removal of vorticity through the western boundary depends on both the viscosity and the gradient of the time-mean vorticity (the vorticity flux is $-\delta_{M}^{3} \nabla \bar{\zeta}$ ). Apparently, with constant viscosity the gradient of the vorticity cannot be large enough to remove the vorticity from the basin at the western boundary alone in the single-gyre.

As the Reynolds number increases, the increase in the vorticity gradient apparently changes the flow dramatically. It is difficult to decide which results of the increase in the vorticity gradient are direct results of gradient and which results are forced secondarily by the direct results, as all of these events are linked in the inertiallydominated equilibrium. The total effect of all of these changes is that the eddy 
delivery of vorticity to the frictional sublayer and the frictional removal of vorticity through the western boundary layer is disrupted when the vorticity gradient becomes too large, an effect I will call the removal crisis. I will propose three conjectures as to how this removal crisis begins, but ultimately the cause of this crisis is the lack of vorticity removal from the basin by friction.

One possible direct result of increasing the vorticity gradient is that it may affect the formation of eddies or the kind of eddies which are present so that the flux divergence of these eddies undergoes a sign reversal. Once this sign reversal occurs, the frictional flux convergence also changes sign to accommodate. As the frictional fluxes are nearly zonal and do not extend far into the basin, changing the sign of the frictional flux convergence implies that the frictional flux through the boundary in that location also changes sign. Once this frictional flux has changed sign, larger vorticity gradients are required elsewhere along the boundary to remove more of the vorticity and these larger vorticity gradient affect the eddies even more, closing the circle of causality in a positive feedback.

Another mechanism relies on the fact that near this sign reversal the boundary current meanders slightly away from the boundary and back again so that the frictional flux convergence reverses in sign (which balances the change in the eddy flux divergence). The meander away from the boundary is located very near where the recirculation gyre approaches the boundary. Thus, it is possible that the mechanism for the removal crisis is initiated by unchecked vorticity input to the recirculation gyre. As more and more vorticity is input by the wind into the recirculation, it becomes stronger and stronger. Once this gyre becomes sufficiently strong, it causes a meander in the boundary current, reversing the sign of the friction flux convergence, which then leads to a sign reversal of the eddies. However, this hypothesis seems somewhat unlikely as the steady-state solutions with large recirculation gyres as in figure 1-2 do not have a reversal in the sign of the friction flux.

Finally, the recirculation gyre strongly interacts with the eddies, and many of the 

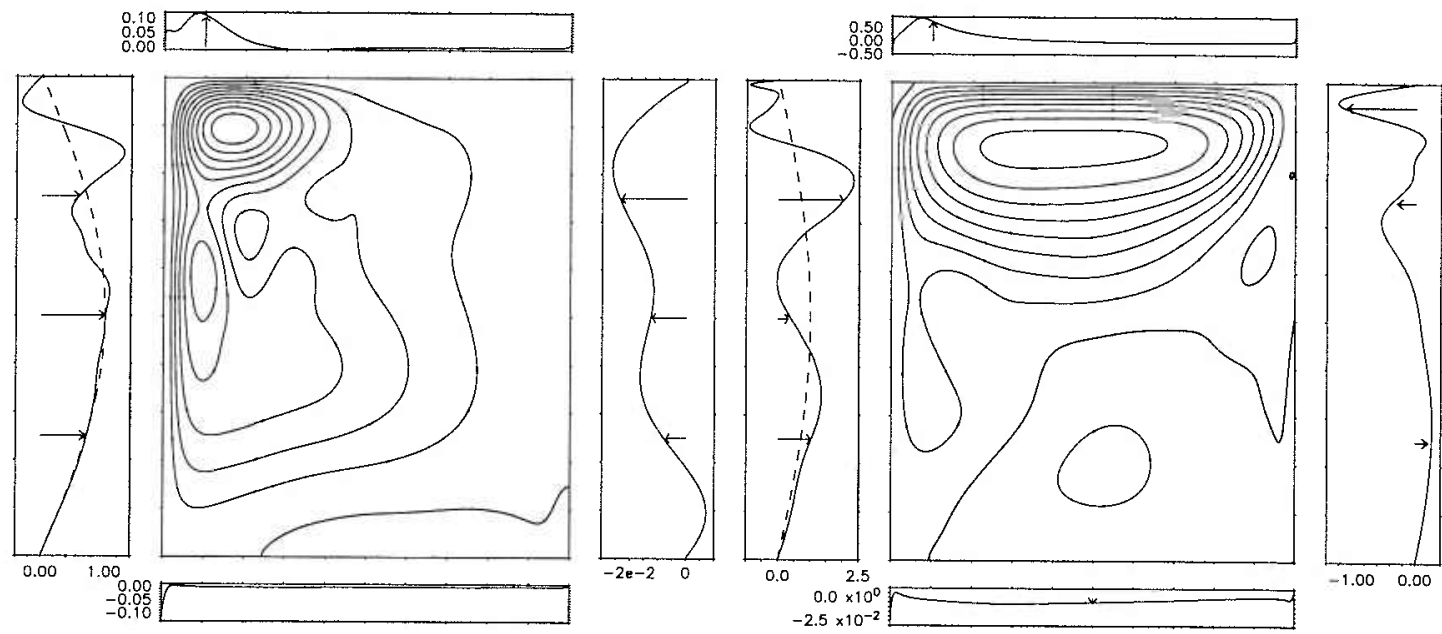

Figure 2-7: Maps of the normal frictional flux through each of the boundaries for the western-intensified $\operatorname{Re}($ bdy) $=0.25, \operatorname{Re}($ int $)=5$ calculation (on left) and the inertiallydominated $\operatorname{Re}(b d y)=5, \operatorname{Re}(i n t)=5$ calculation (on right). The four plots surrounding each contour plot indicate the frictional flux through the nearest boundary to each box $\left(-\delta_{M}^{3} \nabla \zeta\right)$ as a function of distance along the boundary. The flux through the western boundary needed to remove the wind stress input at the same latitude (as in the linear Munk solution) is overlaid with dashed lines. Arrows denote the direction of the frictional flux of positive vorticity. Note that the scales of the flux plots are different.

eddies form along its outer extent. If the recirculation were to grow unchecked, it might eventually reach the point where it changes the eddy field enough to change the sign of the eddy flux divergence. The other effects would follow from this change.

Of course, however this removal crisis at the western boundary is caused, an equilibrium is reached, so the basin-integrated vorticity input by the wind must be able to be removed by friction somewhere. The equilibrium is reached in inertiallydominated calculations when the eastern frictional sublayer provides another source of positive vorticity for the eddies to draw on once the recirculation has grown sufficiently large.

Figure 2-7 shows the frictional flux through the boundary for a western-intensified and an inertially-dominated calculation. Some of the features mentioned above are worth revisiting in the context of this figure. The removal crisis is present in the 
inertially-dominated calculation's flux through the western boundary. A dip in the flux of the western-intensified calculation also occurs near this point, but it does not actually reverse the direction of the flux. Note how the maximum flux through the western boundary is nearly 2.5 times larger in the inertially-dominated case than in the western-intensified one. Because the value of the viscosity at the boundary is 20 times smaller in the inertially-dominated case, this means that the maximum vorticity gradient at the boundary in this calculation approximately 50 times greater than in the western intensified case! The fluxes through the eastern boundary are negligible in the western-intensified case, while the in inertially-dominated case the maximum flux through the eastern boundary is only 2 times smaller than the maximum through the western boundary. Thus, figure 2-7 also clearly shows the removal crisis, the larger gradient of vorticity at the boundary in the inertially-dominated case, and the necessity of the recirculation gyre reaching the eastern boundary in the inertiallydominated calculation.

The frictional flux through the western boundary needed to remove the wind input at the same latitude where it is injected is indicated with dotted lines in figure 27. Clearly, the dip in the frictional flux and the reversal of the frictional flux in the northwest corner make the removal of vorticity occur at a different latitude in the western-intensified calculation. These effects are even more pronounced in the inertially-dominated calculation, where a significant portion of the frictional flux is through the eastern boundary.

Figure 2-8 is a collage of the eddy flux convergences from the same calculations depicted in figure 2-2. It is clear that as Re(int) and Re(bdy) increase, the eddy activity increases. The white region of eddy flux convergence near the western boundary noted in figure $2-6 \mathrm{~d}$ is present in all of the inertially-dominated calculations.

The homoparic solutions in figure 2-2 also have similar eddy flux convergences in figure 2-8. The similarity in convergences does not extend to the scale of the finer features in the divergence field. For example, compare divergences of the $\operatorname{Re}(b d y)=3$, 


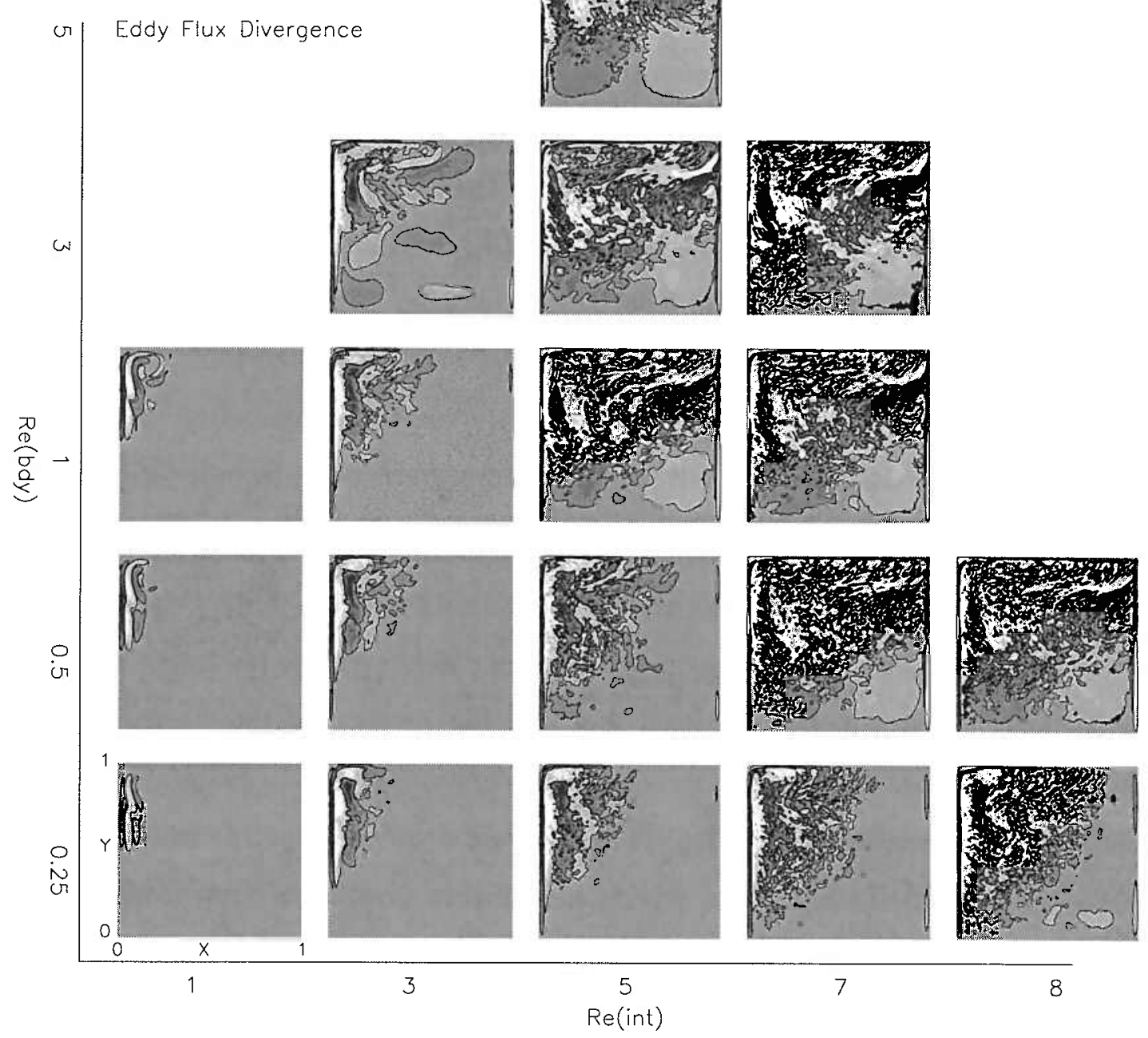

Figure 2-8: Collage of eddy vorticity flux convergences for different calculations. The shading scheme is the same as in figure $2-6$. The -1 and +1 contours of eddy flux divergence are added for contrast. 
$\operatorname{Re}($ int $)=3$ calculation to the $\operatorname{Re}(\mathrm{bdy})=0.25, \operatorname{Re}($ int $)=5$ calculation. While the mean solutions are similar, the eddy flux convergences differ somewhat but the total effect of friction (including the change in the viscosity) compensates. The fine-scale features of the eddy convergence and the eddies themselves differ dramatically. The differences are treated more fully in section 3.2 .

An analysis of the fluxes, instead of the flux divergences, is also enlightening. However, plotting the eddy fluxes of vorticity as a vector field is not particularly helpful, as the vectors do not obviously point from one divergent region to a convergent one. To make the flux field seem more rational, past authors have chosen to decompose the eddy fluxes into divergent and rotational parts. However, as shown in Fox-Kemper et al. (2003) this decomposition is not unique in a bounded domain. One can study the effects of only the divergent fluxes, as they are well defined if they are calculated as the average flux out of a chosen region. As shown in equation 2.15, this is particularly useful when the area of integration is chosen to be the area within a mean streamline, as in that case the mean advection terms vanish. In figure 2-9, the results of such a calculation are shown for two different runs of the numerical model. ${ }^{8}$

Figure 2-9a shows the frictional and eddy fluxes through a mean streamline for the same calculation as shown in figure $2-6 \mathrm{a}$ which is western-intensified. For $\bar{\psi}<0.2$, the eddy fluxes carry more of the vorticity flux. So, in this western-intensified solution, the wind input is carried from the interior streamlines to the region near the boundary (where $\bar{\psi}<0.2$ ) by the eddies. Near the boundary, the eddies deliver the vorticity to the friction which then removes it from the basin.

Figure 2-9b shows the frictional and eddy fluxes through a mean streamline for the same calculation as shown in figure $2-6 \mathrm{~b}$ which is inertially-dominated. In this calculation the maximum of the streamfunction is much larger, as the circulation is inertially-dominated and is much stronger. However, even here the eddy flux of vorticity is larger than the frictional flux for $\bar{\psi}>0.5$. Thus, in the inertially-dominated

\footnotetext{
${ }^{8}$ The reader may wish to review section 2.2 .1 to help interpret these plots of flux versus time-mean streamfunction.
} 

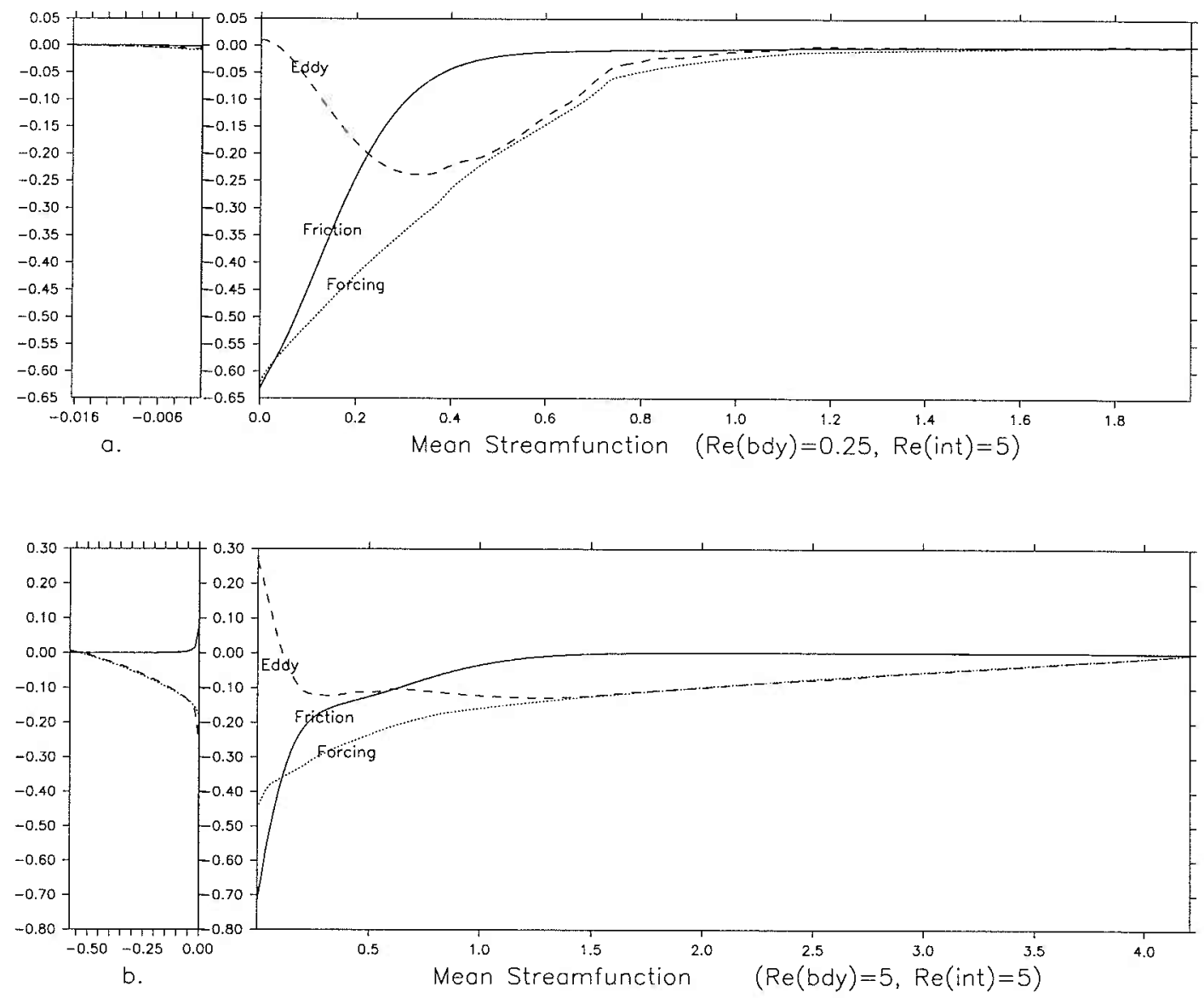

Figure 2-9: (a-b) show the vorticity flux across mean streamlines. Shown are the eddy flux out of the streamline $\left(\oint_{\psi_{c}} \overline{\mathbf{u}^{\prime} \zeta^{\prime}} \cdot \hat{\mathbf{n}} d s\right)$, the friction flux out of the streamline $\left(-\oint_{\psi_{c}} \delta_{M}^{3} \nabla \bar{\zeta}=\hat{\mathbf{n}} d s\right)$, and the wind forcing within that streamline $\left(-\iint_{\psi \leq \psi_{c}} \sin (\pi y) d^{2} A\right)$. A balanced budget results when the eddy flux plus the friction flux equals the forcing. (a) shows the result for the $\operatorname{Re}(\mathrm{bdy})=0.25, \operatorname{Re}($ int $)=5$ calculation. (b) shows the result for the $\operatorname{Re}(\mathrm{bdy})=5, \operatorname{Re}(\mathrm{int})=5$ calculation. See figure 2-6 for location of mean streamlines for these calculations. The sum of the frictional fluxes at the boundary is the frictional removal from the basin. The sum of the eddy fluxes at the boundary is zero. See section 2.2.1 for details. 
calculations it is not a lack of eddy vorticity transport which leads to the inertialdomination. Rather, it is the lack of friction near the boundary which prevents the ultimate removal of the vorticity from the basin.

In thermal convection, the Nusselt number is a ratio of heat fluxes used to assess the increase in efficiency of heat transport due to the presence of convection. It is defined as the ratio of the total heat transport to what the purely diffusive heat transport would be for the same flow. Thus, if there is no convection, the ratio is one. If there is convective transport, this ratio will be in excess of one. The more efficient the convective transport, the larger this ratio will be. That is, if the convection is able to make a large transport even with a small gradient, the Nusselt number is large, and the convection is considered efficient.

The fluxes determined in figure 2-9 allow a similar construction to the Nusselt number to be made from the vorticity fluxes. The ratio of the total vorticity transport across a mean streamline to the frictional flux across that mean streamline, like the Nusselt number, is a measure of how efficiently the eddies are able to flux vorticity across the mean streamlines. For the purposes of this dissertation, the efficiency of the eddy transport will be defined as the ability of eddy fluxes to increase the magnitude of this ratio, and thereby more efficiently deliver vorticity to the frictional sublayer. Note that for a steady-state solution, this ratio will be one everywhere. Otherwise, the larger it is, the more important the eddy flux is across a particular streamline. If it is less than one, it is because the eddy flux is in the opposite direction to the frictional flux. So, just as with the Nusselt number, if this flux ratio is large, the eddies are transporting a large flux compared to what the frictional flux, even though the mean gradient is not large enough to cause a large frictional flux, so the eddy flux is considered to be efficient.

The Nusselt number is usually defined with a denominator that is the frictional or diffusive flux which would occur in the absence of motion. Here, however, the denominator of the flux ratio depends on the time-mean solution's vorticity gradient which 
changes throughout the basin and as the parameters change. Because the Nusselt number is compared to a 'standard' flux which doesn't change with the parameters, the Nusselt number is easier to interpret than this flux ratio.

The prototypical Nusselt problem is a fluid trapped between an upper and a lower surface, each at a particular temperature. In that situation, it is clear that the heat flux from one plate to another is well-defined. It is sensible to compare the case without convection to the case with convection, as the plates do not move, nor does the temperature of each plate. There is no mean flow which changes with the parameters to include.

The flux ratio used here, on the other hand, is not as easy to define or interpret because the mean flow and the source of vorticity change with the parameters. To remove the effects of the changing mean fluxes from the ratio, we consider the flux across mean streamlines. The result of this, however, is that unlike the two fixed plates in the Nusselt problem, the source of vorticity changes as the mean streamlines change and enclose more or less of the wind input. In the Nusselt problem, the flux across a plane parallel to the two plates at any vertical level is the same in equilibrium. Here, the flux across the innermost nested streamline is small (as it encloses little of the wind input) while the flux across the streamline closest to the boundary is large (as it includes almost all of the wind input). Thus, the flux across each streamline is different and cannot be compared to a standard value of the flux as in the Nusselt problem. Thus, the flux ratio here needs to be a function of the mean streamfunction, which leads unavoidably to a variable denominator. Although this flux ratio is therefore not as satisfying as the traditional Nusselt number (and should not be directly compared to Nusselt numbers in other calculations for this reason), it seems the best choice available.

A plot of this ratio is given in figure 2-10 for the same calculations shown in figure 2-9. It is clear from figure 2-10 that the calculation with boundary-enhanced viscosity has more of its total vorticity flux across mean streamlines carried by the 


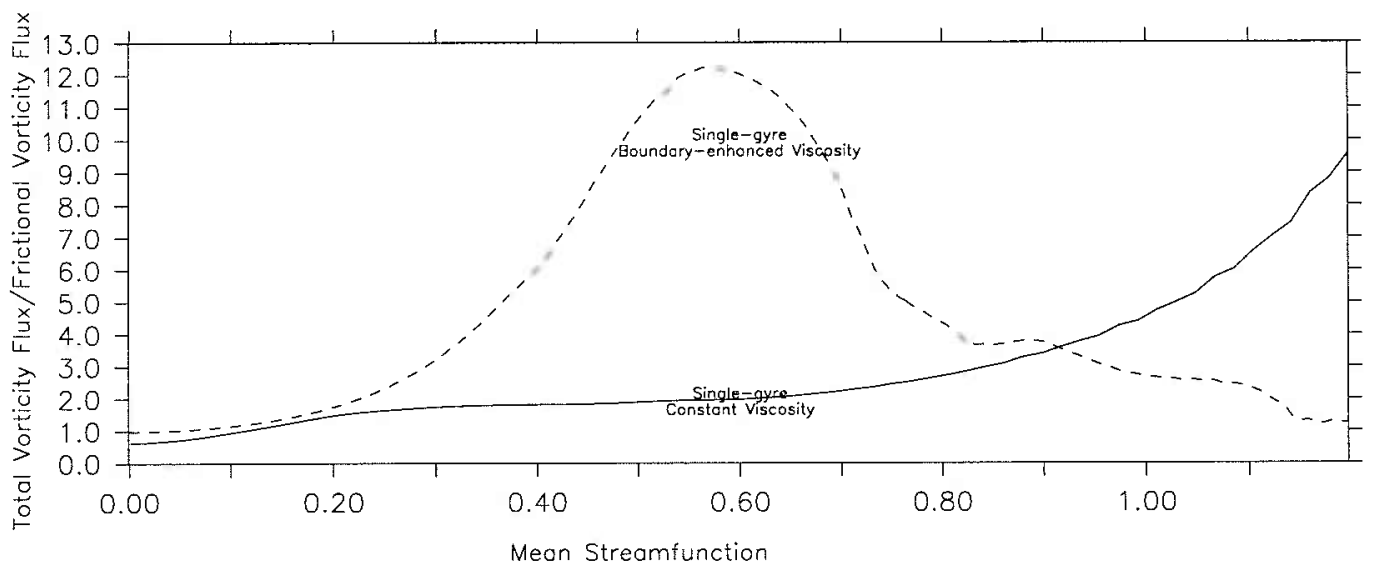

Figure 2-10: This figure shows the ratio of the total vorticity flux across a mean streamline to the frictional flux across a mean streamline as a function of mean streamfunction. It is constructed from the fluxes in figure 2-9.

eddies in the basin interior. For both calculations, the ratio approaches one as $\bar{\psi}$ approaches zero as there is no eddy flux through the basin boundary. ${ }^{9}$ Thus, the eddies are able to control the circulation strength in the boundary-enhanced viscosity case while they are not able to do so in the constant viscosity case.

To understand what separates the inertially-dominated and the western-intensified equilibria, it is helpful to see the changes in the meridional averages of the terms in the vorticity equation with Reynolds number. Figure 2-11a shows the viscosity parameter used in three separate calculations which share the same value of $\operatorname{Re}(b d y)$.

Figures 2-11b-d show the effect of changing the viscosity on the meridional average of the vorticity flux convergences. Of the three calculations, the two with the largest interior viscosity are western-intensified, while the other one is inertially-dominated. Figure 2-12a shows the viscosity parameter used in three separate calculations, while figures $2-12 \mathrm{~b}-\mathrm{d}$ show the effect of changing the viscosity on the meridional average

\footnotetext{
${ }^{9}$ The eddy flux across the zero mean streamline is nonzero due to the flux into the counterrotating region. This is due to the way that the fluxes across mean streamlines are defined in this figure, as discussed in section 2.2.1.
} 


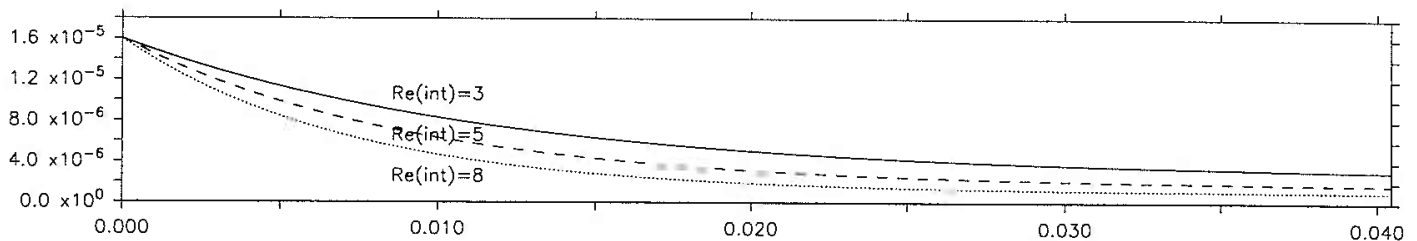

a) Viscosity Parameter

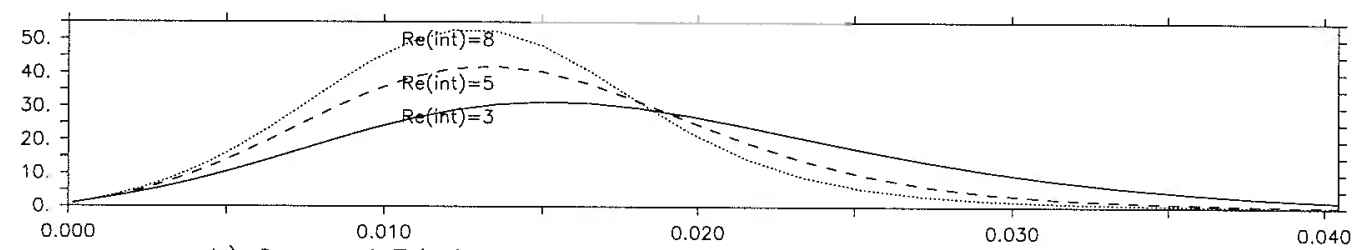

b) Conv. of Friction Flux

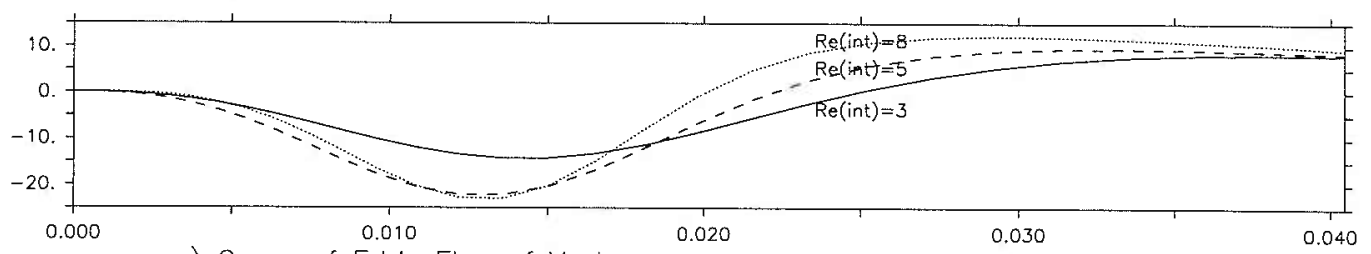

c) Conv. of Eddy Flux of Vort.

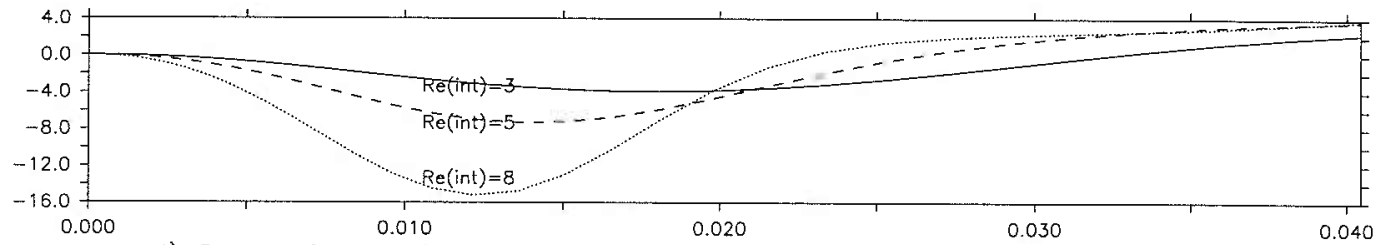

d) Conv. of Mean Flux of Rel. Vort.

Figure 2-11: (b-d) show the meridional average from $y=0$ to $y=1$ of the vorticity flux convergences as a function of $x$ within the frictional sublayer for solutions with three different values of $\operatorname{Re}(\mathrm{int}): 3$ (solid lines), 5 (dashed lines), and 8 (dotted lines). $\operatorname{Re}(\mathrm{bdy})=0.5$ for all three cases. (a) shows the three different viscosity parameters $\left(\delta_{M}^{3}\right)$ used. (b-d) show the frictional, eddy and mean flux convergences, respectively. The $\beta$-flux is similar across the three calculations in this region (not shown). In the inertially-dominated solution $(\operatorname{Re}(\mathrm{bdy})=8$ (dotted)) the mean advection delivers negative vorticity closer to the boundary than the eddy advection, while in the western-intensified cases (Re(int) $=3$ (solid) and $\operatorname{Re}($ int $)=5$ (dashed)) the eddies deliver negative vorticity closer to the boundary. 


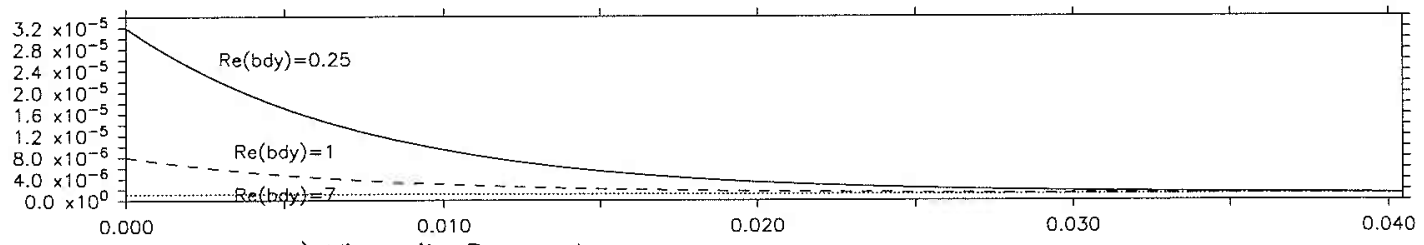

a) Viscosity Porameter

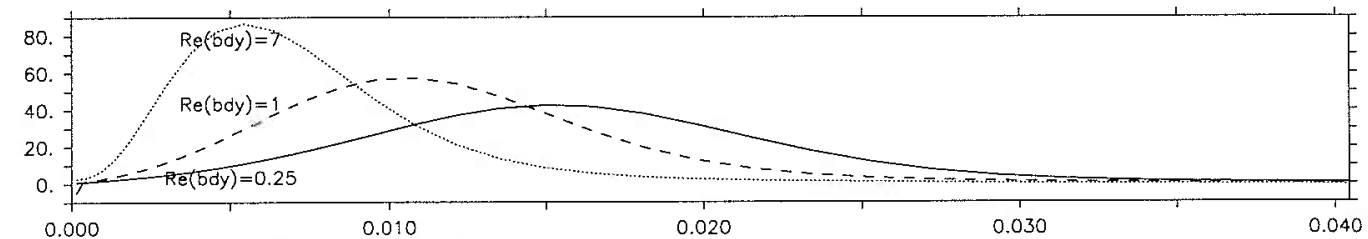

b) Conv. of Friction Flux

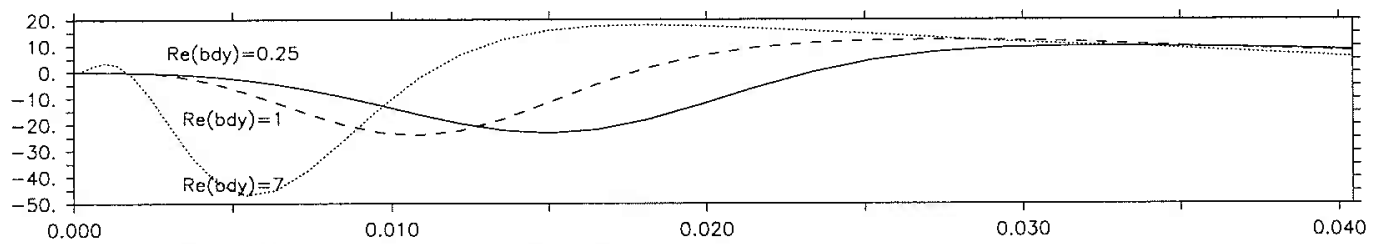

c) Conv. of Eddy Flux of Vort.

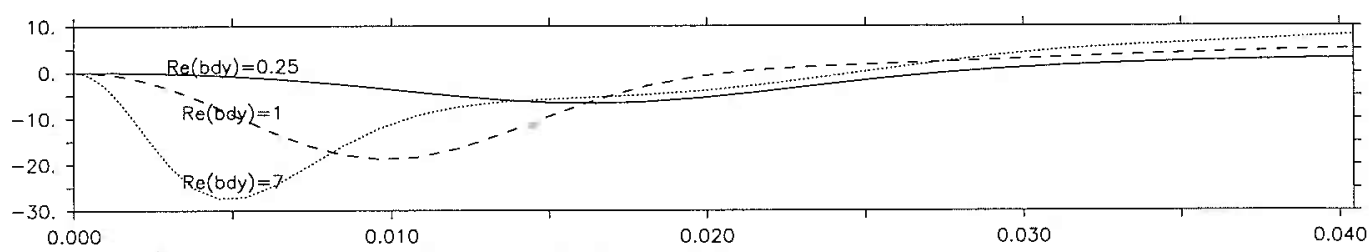

d) Conv. of Mean Flux of Rel. Vort.

Figure 2-12: (a-d) are as in figure 2-11 except for solutions with three different values of $\operatorname{Re}(\mathrm{bdy}): 0.25$ (solid lines), 1 (dashed lines), and 7 (dotted lines). Re(int) $=7$ for all three cases. In the inertially-dominated solutions $(\operatorname{Re}(b d y)=1$ (dashed), 7 (dotted)) the mean advection delivers negative vorticity closer to the boundary than the eddy advection, while in the western-intensified case $(\operatorname{Re}(b d y)=0.25$ (solid)) the eddies deliver negative vorticity closer to the boundary. 

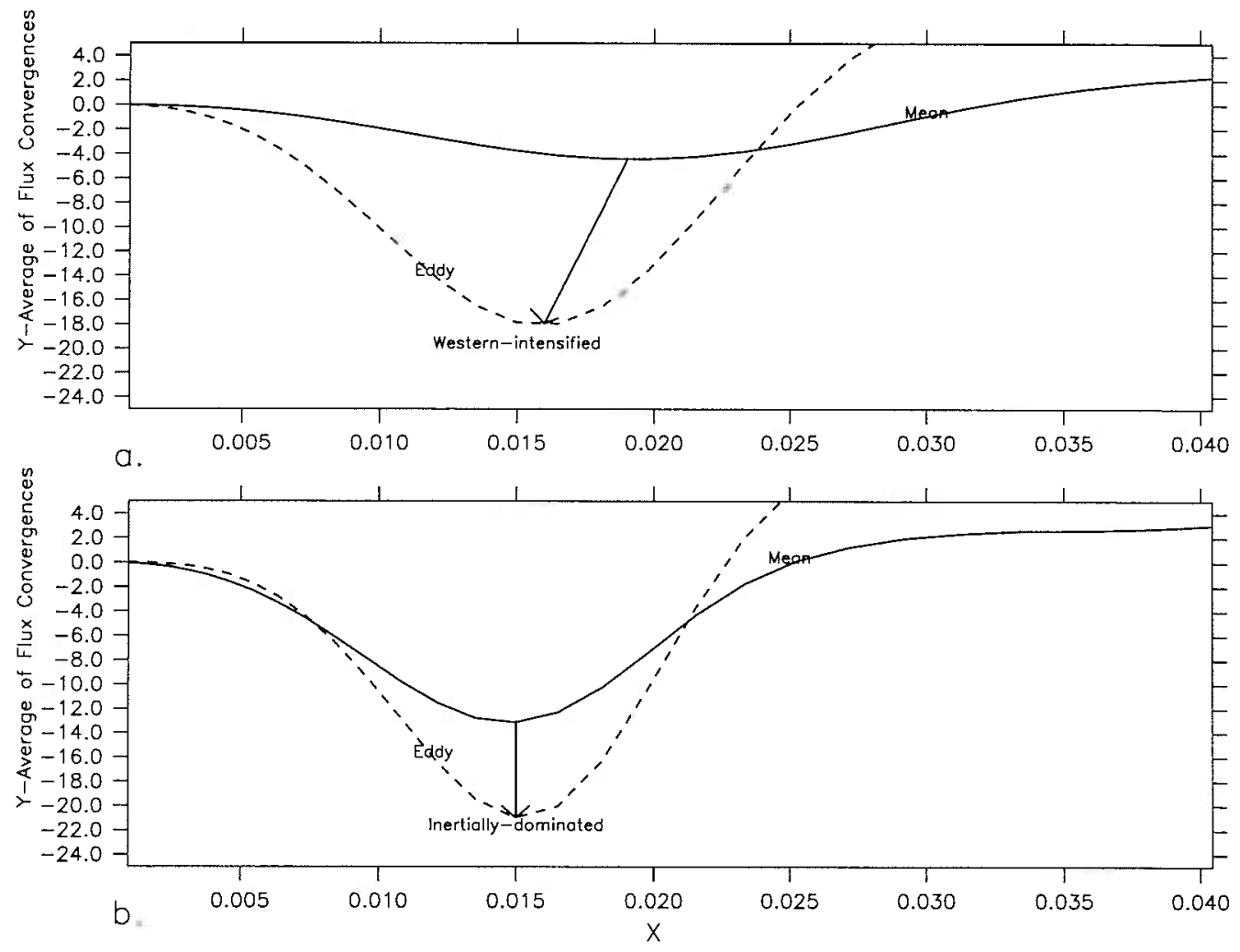

Figure 2-13: (a-b) show the average from $y=0$ to $y=1$ of the eddy and mean vorticity flux convergences as a function of $x$ within the frictional sublayer for two different solutions: (a) $\operatorname{Re}(\mathrm{bdy})=0.25, \operatorname{Re}($ int $)=5$ (western-intensified), and (b) $\operatorname{Re}(\mathrm{bdy})=$ $0.25, \operatorname{Re}($ int $)=9$ (inertially-dominated). In the inertially-dominated solution the mean advection delivers negative vorticity closer to the boundary than the eddy advection, while in the western-intensified case the eddies deliver negative vorticity closer to the boundary. 
of the vorticity flux convergences. Of the three calculations, only the one with the largest boundary viscosity is western-intensified, while the other two are inertiallydominated.

The balance is similar in the six calculations shown in figures $2-11$ and 2-12, with friction, eddy and mean vorticity flux convergences all playing important roles in the frictional sublayer. The $\beta$-flux convergence is also an important term but does not vary significantly with viscosity, so it is not shown.

The relative location of the peak of the eddy and mean flux convergences is different for the western-intensified and the inertially-dominated calculations. Where the solution is inertially-dominated, the mean transport peaks closer to the boundary than the eddy transport, while western-intensified calculations have the eddy advection delivering the vorticity deeper into the frictional sublayer. It is sensible that in a parameter range where the steady-state calculation is inertially-dominated but the time-dependent calculation is western-intensified that the eddies should be able to deliver vorticity closer to the boundary than the mean flow. Otherwise, the eddies would be no more effective in reducing the circulation strength than the mean flow. The predictive skill of this simple test holds true throughout the calculations carried out here, and it is directly related to the region of eddy flux convergence pointed out in figure 2-6d. As the friction is insufficient to remove all of the vorticity delivered to the frictional sublayer, the eddies reverse their flux in this region and transport vorticity back toward the basin interior. This effect, when averaged, is what causes the peak of eddy vorticity flux convergence to be located farther out than in the case where the friction is sufficient. Figure 2-13 compares the location of the peaks in an inertially-dominated versus a western-intensified calculation.

Thus, the delivery of vorticity into the frictional sublayer by the eddy fluxes breaks down in the inertially-dominated solutions. This is shown on average in figures 2-11, 2-12, and 2-13 and is also present in figures 2-6 and 2-8. In the inertially-dominated solutions near the center of the frictional sublayer, the eddy flux divergence changes 
sign because the frictional sublayer does not remove enough of the vorticity (see especially figure 2-6d).

In summary, eddy fluxes are the primary transport of vorticity across mean streamlines transport throughout the basin. Only in the frictional sublayer is the friction dominant. If the frictional sublayer is sufficiently strong, the eddies are able to flux vorticity deeper into the sublayer than the mean flow can. This flux prevents the solution from entering an inertially-dominated configuration. Therefore, the eddies can replace the frictional flux across mean streamlines in the basin interior as the viscosity is lowered. Occasionally, with smaller and more energetic eddies in solutions with higher $\operatorname{Re}($ int), the mean flow of solutions with different $\operatorname{Re}(\mathrm{int})$ an $\operatorname{Re}(\mathrm{bdy})$ can be made similar (homoparic), where the increase in the eddies and the decrease in friction manage in concert to produce a similar effect on the mean flow.

\subsection{Conclusion}

In this chapter, the well-known result that the eddies in a wind-driven, single-gyre model are unable to accommodate a reduction in the viscosity everywhere in the basin is confirmed. This is because although the eddies are capable of replacing the frictional fluxes across mean streamlines in the basin interior, they are unable to flux vorticity through the basin boundaries.

Because the friction is intended in part to parameterize the effects of eddies, this result has caused much confusion in the past. It was thought that as the parametrization was removed, the newly energized eddies would be able to take over for the parameterization. Because this does not occur, the increasing domination of inertial terms throughout the basin is sometimes called inertial runaway.

However, in this chapter, it is pointed out that the friction in the homogeneous model represents not only the eddies, but also the unresolved interactions with the boundary. In particular, it represents all of the processes that might ultimately 
remove vorticity from the basin. By using a constant viscosity, one tacitly assumes that these processes are equivalent to the unresolved eddy processes. The results in this chapter demonstrate that if a separation is made between the boundary processes and the interior processes, the conundrum of inertial runaway is avoided.

When the viscosity is large in only a narrow frictional sublayer, even one which is thinner than the width of the inertial boundary layer, but small in the basin interior, the circulation can be controlled with a resulting equilibrium state which is westernintensified. The mechanism of this control relies upon the efficient removal of the wind's vorticity input from the basin. The removal of this vorticity occurs in steps. First, the vorticity is absorbed from the wind primarily by the mean flow. As the viscosity is small in the basin interior, the mean flow is relatively inviscid, and so it carries the vorticity along to the western boundary. The western intensification model presented by Charney is quite similar to the concentration of the vorticity into a thin boundary layer which occurs in this model. Because the frictional sublayer is smaller than the inertial boundary layer, some of the mean flow passes through the frictional sublayer, but some of it does not. The eddies are able to take the vorticity in the mean flow which doesn't penetrate this frictional sublayer and transport it across the mean flow's streamlines closer to the boundary and into the frictional sublayer where they deposit it. Thus, the effect of the eddies and of the mean flow is not to remove vorticity, but to transport it. Finally, the friction removes the vorticity from the basin.

When the viscosity is too small in the frictional sublayer, the friction cannot remove all of the vorticity that the eddies deposit within only the western and northern frictional boundary layers. Thus, in an area of the frictional sublayer, the eddies' vorticity transport reverses direction and transports negative vorticity toward the interior. The buildup of negative vorticity in the recirculation gyre causes the recirculation to grow until the eastern boundary is reached, at which point the strong mean flow of the recirculation gyre against the eastern boundary's frictional sublayer 
is able to provide an additional source of vorticity to oppose the wind's input.

Ultimately, these are consequences of the fact that the removal of vorticity through the western boundary relies on both the viscosity and the gradient of the time-mean relative vorticity (the vorticity flux is $\delta_{M}^{3} \nabla \bar{\zeta}$ ). Thus, as the viscosity near the boundary decreases, the relative vorticity gradient increases. This gradient is active in the inertial terms as well, and a consequence, the eddy delivery of vorticity to the frictional sublayer is interrupted. When this occurs, the eddies are not able to deliver the vorticity any closer to the boundary than the mean flow, and so the interior streamlines of the mean flow have no outlet for the vorticity input by the wind. Only when the recirculation gyre reaches the eastern boundary and a new source of vorticity is found is an equilibrium made possible. From this point on, a change in circulation strength at equilibrium with increasing Reynolds number is reduced. However, the recirculation gyre now fills most of the basin, and so the equilibrium is inertiallydominated.

Thus, when the frictional sublayer is sufficiently strong, the solutions can be controlled. The primary effect of friction in the interior, transport of vorticity across mean streamlines, can be replaced by eddies. Sometimes, when the Reynolds number changes, the changes in the eddies and those in the friction compensate so that the mean flow is relatively unchanged. These homoparic solutions lend great hope that there may be a truly accurate eddy parametrization possible for this model.

The importance of these results in the context of the real ocean is that although eddies in the ocean interior may transport vorticity, they cannot create or destroy it. Therefore, there will always be a role for boundary processes.

Also, there is little reason to believe that inertial-domination will not occur in more inclusive ocean models eventually. General circulation models cannot be operated with viscosities small enough for this to be a problem now, but this will be possible soon. Then, it will be time to turn not to the strengthening of eddy processes in the interior, but to the interaction of eddies and the boundary. The appropriate 
interpretation of the results here is that the eddies produced in this model will have analogues in more complex models. It seems likely that the additional sources of instabilities that lead to eddies in more complex models should make it easier for the eddies to perform the transport of vorticity across mean streamlines. Thus the ability of the simple eddies present here to effect enough vorticity transport indicates that even in the general circulation models it is likely that inertial-domination be controlled by ensuring the boundaries are accurately parameterized with sufficient capability for vorticity removal.

This model, though simple, has been able to point out the importance of the structure very near the boundary and its role in the removal of vorticity from the basin. As general circulation models reach higher Reynolds numbers and finer resolution, these issues will become relevant in understanding the results of those more complex models as well. When these models are well-understood enough to correctly predict the transports and recirculations of the western boundary currents without tuning of parameters it is now clear that the interaction of eddies with the boundary will play a role. 


\section{Chapter 3}

\section{Eddies' Effects in the Single-Gyre Model with Low Interior Viscosity}

The previous chapter demonstrates that the circulation strength of the single-gyre can be controlled at a larger Reynolds number using a combination of eddy transport and boundary-enhanced viscosity. Once this control is achieved, it is possible to produce calculations which remain western-intensified at a much higher Reynolds number in the basin interior. In these relatively inviscid calculations, a number of interesting phenomena occur. Although many deserve more attention than is possible here, brief introductions to those phenomena that I have found interesting are in this chapter. In particular, much of the interest present in this model is created by the existence and the effects of strong eddies. Along with these introductions, two sections-one on the effects of bottom friction and one on changing the boundary conditions to slip-help to place the results of this model in context with the work of others and the next chapter.

The structure of the boundary layer in the main region of eddy activity is explored in section 3.1. The structure of the boundary layer in this region is novel, with the eddies playing a critical role.

The homoparic solutions that were observed in the results of the first chapter are 
explored in more detail in section 3.2. Some diagnostic calculations reveal that these homoparic solutions are not exactly the same, in particular the energetics and scales of the eddies are quite different.

The counter-rotating gyres that were pointed out in the less viscous calculations in the previous chapter are diagnosed in section 3.3. The eddies again play a critical role.

The eddies form a complicated temporal structure for the calculations which is discussed in section 3.4. Although they are in a equilibrated state, some of the solutions have a large degree of variability, and they can be variable on rather slow time scales.

The presence of variability that resembles the linear basin modes of oscillation is noted in section 3.5 .

In the previous chapter, the role of eddies and friction in the removal of vorticity was presented. In section 3.6, the complementary removal of energy by the eddies and friction is discussed.

The final sections of this chapter present some of the effects of adding bottom friction in addition to the lateral friction and using slip boundary conditions on the eastern and western boundaries. The eddies in the model are significantly altered by these changes.

\subsection{Boundary Layer Balances}

In order to compare the solutions generated here with traditional boundary layer theories (such as Stommel (1948), Munk (1950), and Charney (1955)), the balance of terms in the vorticity equation is presented near the western boundary. The three plots in figure 3-1 show the meridional average of the terms in the vorticity equation, with the average taken over different regions of the boundary layer. These regions are indicated in figure 3-2. Figure 3-1a shows the average in the region where the interior 


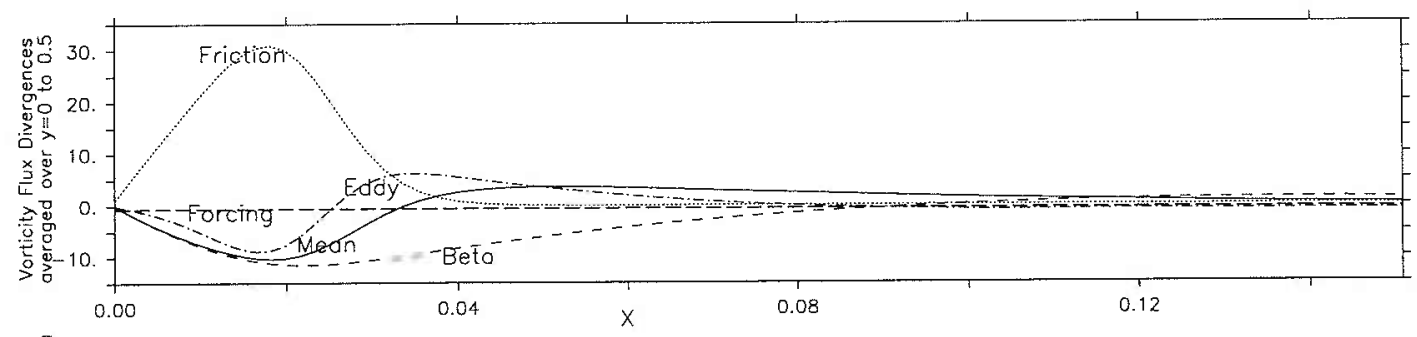

a

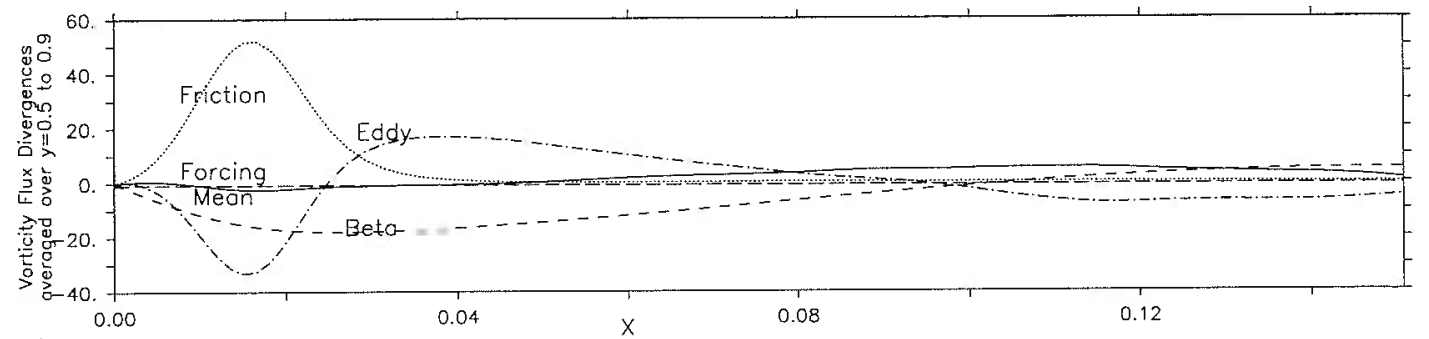

b.

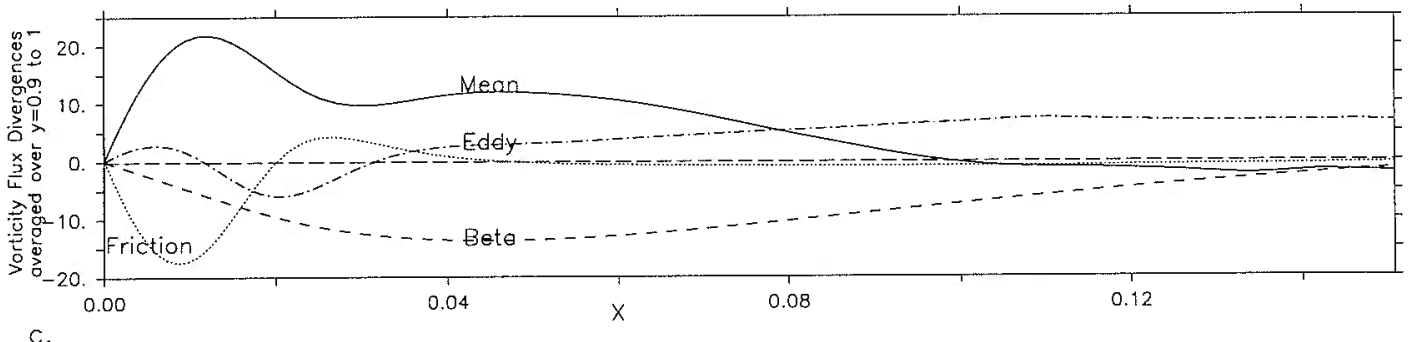

Figure 3-1: (a-c) show the meridional average of the vorticity flux convergences as a function of $x$ for the $\operatorname{Re}(\mathrm{bdy})=0.25, \operatorname{Re}(\mathrm{int})=5$ calculation. (a) shows the average over the Charney boundary layer region where the interior flow enters the boundary current ( $y=0$ to 0.5 ). (b) shows the average over the region between the Charney region and the exit region. (c) shows the average over region where the boundary current leaves the western boundary. The averaging regions are indicated in figure 3-2.

flow enters the boundary layer. Figure 3-1b shows the average in the region between where the interior flow enters the boundary layer and the exit region. Figure 3-1c shows the average in the region where the boundary current exits to rejoin the interior flow.

Figure 3-1a shows the meridional average of terms in the vorticity equation in the region where the interior flow enters the boundary layer. In this region, the Char- 


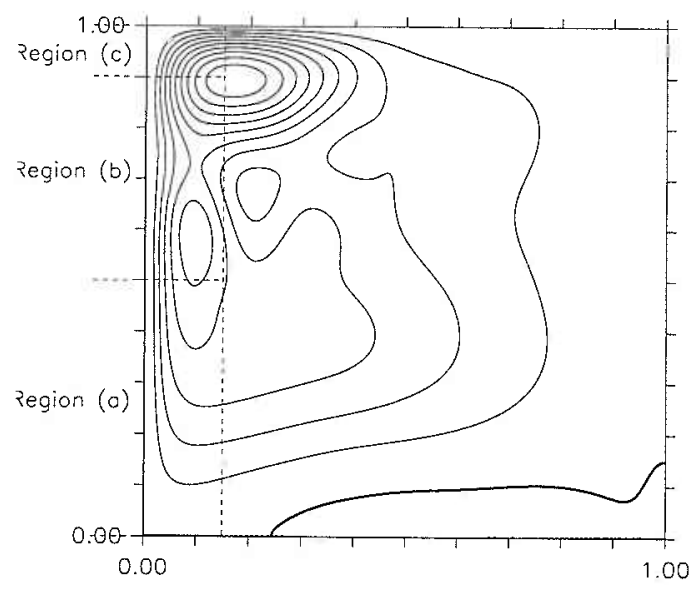

Figure 3-2: Contours of the time-mean streamfunction of the $\operatorname{Re}(\mathrm{bdy})=0.25$, $\operatorname{Re}($ int $)=5$ calculation with the regions of the boundary layer that are analyzed in figure 3-1 indicated.

ney (1955) inertial boundary layer solution can exist (as demonstrated by Greenspan (1962)), and for the outer part of the boundary current (where the $\beta$-term still indicates a strong northward flow, $0.04<x<0.08$.) the calculation's balance resembles the Charney (1955) model as the advection of planetary vorticity and relative vorticity are in balance. Although the Charney boundary layer width is 0.02 , this is only the a priori estimate of the exponential scale of the boundary current, the vorticity balance the Charney model proposes is important over a somewhat wider region. For $x<0.03$, there is a frictional sublayer acting to enforce the no-slip boundary condition. There, the friction balances the mean advection of both relative and planetary vorticity. In the middle region, $0.03<x<0.05$, there is significant action of eddies. The eddies widen the influence of the frictional sublayer to this region by transporting vorticity to the frictional sublayer. In essence, the solution in the entry region of the boundary layer agrees with the Ierley and Ruehr (1986) steady-state picture of an inertial boundary layer with a frictional sublayer, except that the eddies join in to 
widen the influence of the frictional sublayer toward the interior.

Figure 3-1c shows the meridional average of terms in the vorticity equation in the exit region of the boundary layer, where it combines with the recirculation gyre. As shown by Il'in and Kamenkovich (1964) (and in English in Kamenkovich (1966)) and Ierley and Ruehr (1986), there is no steady-state boundary layer solution here. In this region, the dynamics are dominated by the mean flow toward the interior. The primary balance is between the advection of relative and planetary vorticity. For $0.08<x<0.15$, the eddy flux convergence is balanced by all of the other terms combined. There is a frictional sublayer present here as well, except in this region, the friction is acting to add negative vorticity to the basin, rather than to remove it. This appears to be a necessary consequence of the transition in this region from noslip to slip boundary conditions. In the next chapter, we will see that this transition has significant consequences in the structure of the boundary layer.

Figure $3-1 b$ shows the meridional average of terms in the vorticity equation in the region between the entry and exit region. Here, the boundary layer structure is unlike any of the traditional steady-state theories. Like the ideas of Pedlosky (1965a), the presence of time-dependent phenomena near the boundary is important, but here the critical feature is not the presence of reflecting remotely-forced Rossby waves; these eddies are locally generated by shear instabilities. The eddies play a critical role in energy and vorticity transport in this region, and they are present because the vorticity build-up near the boundary leads to shear instabilities.

Edwards and Pedlosky (1998) find that a similar boundary layer structure is possible when mass needs to be transported across the equator in a relatively inviscid boundary layer. In their model, a vorticity transformation is required for the flow to cross the equator. Edwards and Pedlosky (1998) find that at sufficiently large Reynolds number, the necessary transformation of vorticity is carried out by the combined effects of eddies and friction. The eddies transport vorticity from an relatively inviscid boundary layer to a frictional sublayer. The removal of the vorticity 
from the boundary layer fluid by the eddies changes the vorticity of the boundary layer fluid as it progresses.

Toward the interior in figure 3-1b, the balance is between eddy advection of vorticity and the advection of planetary vorticity, and toward the boundary there is a frictional sublayer. This region could be described as having an eddy-inertial boundary layer with a frictional sublayer, or perhaps as having a turbulent frictional boundary layer. In this region of the boundary layer the change in vorticity due to the northward advection of planetary vorticity is relieved by the eddy vorticity flux convergence.

To summarize, the boundary layer structure in the single-gyre model with boundaryenhanced viscosity has a clear separation into an outer inertial region and an inner frictional sublayer. Where a steady-state inertial boundary layer solution exists, this inertial region is of this type. In this part of the boundary layer, the eddies work to widen the influence of the direct action of friction. Farther along the boundary layer, the steady-state solution no longer exists, and in this region the eddies play a primary role-they balance the $\beta$-term single-handedly.

\subsection{Homoparic Calculations}

In section 10, it is pointed out that some of the solutions with different values of $\operatorname{Re}($ int $)$ and $\operatorname{Re}(\mathrm{bdy})$ have very similar mean fields. In this section, two pair of these homoparic calculations are examined closely. The first pair contains the $\operatorname{Re}(b d y)=0.5$, $\operatorname{Re}($ int $)=3$ calculation and the $\operatorname{Re}(\mathrm{bdy})=0.25, \operatorname{Re}($ int $)=5$ calculation. The second pair contains the $\operatorname{Re}(b d y)=3, \operatorname{Re}($ int $)=3$ calculation and the $\operatorname{Re}(b d y)=0.25, \operatorname{Re}($ int $)=8$ calculation.

Figure 2-2 shows that the two members of each pair have quite similar time-mean streamfunctions. The vorticity dynamics of the time-mean fields are therefore quite similar as well, although there are small differences. On the other hand, the eddies present in the homoparic calculations differ significantly between the pair members. 
This section demonstrates that these solutions have only small differences in the mean fields, while the frictional flux divergence (including the effects of changing the viscosity) and eddy vorticity flux divergence differ slightly in a compensating way, and while the eddies themselves are quite different.

Figure 3-3 shows the meridional averages of the terms in the vorticity equation near the western boundary for the two homoparic pairs. In each pair, the meridionally averaged vorticity flux convergences are similar. The small differences that do appear in the flux convergences can be divided into two general groups, those whose magnitudes vary and those whose gradients vary. An example of each is shown here.

In figure $3-3 b$, all of the terms are similar except the eddy and frictional terms which change in magnitude as $\operatorname{Re}(b d y)$ and $\operatorname{Re}($ int $)$ change. In the $\operatorname{Re}(b d y)=0.25$, $\operatorname{Re}(\mathrm{int})=5$ calculation, the eddies have a larger magnitude of vorticity transport than in the other case. The frictional term also increases magnitude modestly to accommodate this change in the eddy fluxes. Thus, although the eddy fluxes change, the net effect of the eddy fluxes and frictional fluxes on the mean flow does not. Because the frictional term is proportional to a higher derivative of the streamfunction than the mean advection or $\beta$-term, this adjustment can be made without a significant change to the other terms. A small change is present in the $\beta$-term, which indicates that the boundary current is slightly wider in the $\operatorname{Re}(\mathrm{bdy})=0.25, \operatorname{Re}($ int $)=5$ case than in the $\operatorname{Re}(b d y)=3, \operatorname{Re}(i n t)=3$ case. The differences between the members of this homoparic pair are typical of homoparic pairs where the $\operatorname{Re}(b d y)$ and $\operatorname{Re}(i n t)$ changes are not large.

Figure 3-3a shows a homoparic pair where the changes in $\operatorname{Re}(\mathrm{bdy})$ and $\operatorname{Re}($ int $)$ are larger than those in the homoparic pair shown in figure $3-3 \mathrm{~b}$. In figure $3-3 \mathrm{a}$ it is the gradients in the mean fields, rather than the magnitudes which are different. This can be understood by noting that since the same vorticity flux must be transported by the frictional term as the boundary is reached, there must be a change in the mean field so that $\delta_{M}^{3} \nabla \zeta$ remains the same at the boundary. In figure $3-3 \mathrm{a}$, the $\operatorname{Re}(\mathrm{bdy})=0.25$, 


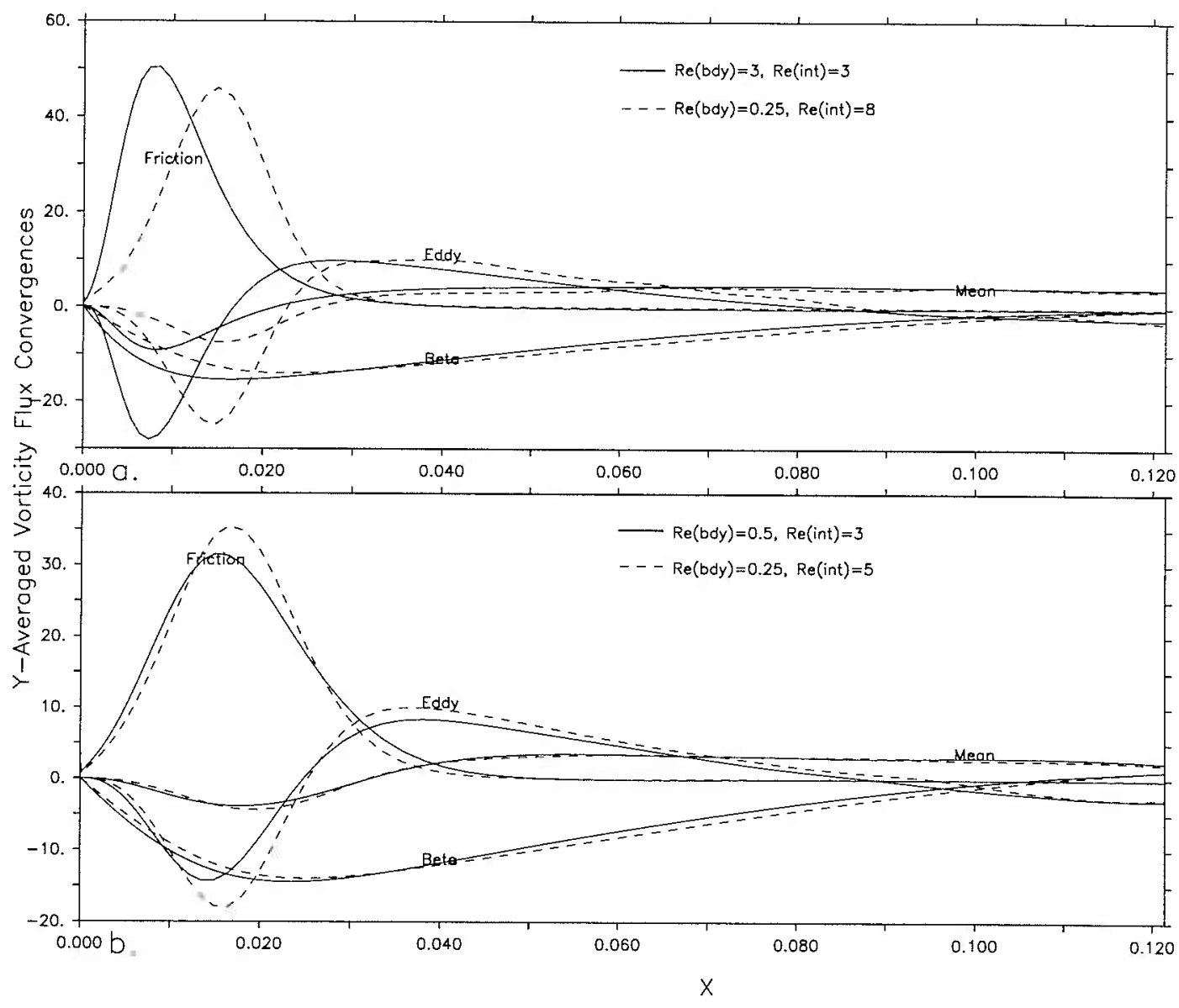

Figure 3-3: ( $a-b)$ show the meridional average of the vorticity flux convergences in the Reynolds-averaged vorticity equation for two pair of homoparic calculations. (a) shows results from the $\operatorname{Re}(\mathrm{bdy})=3, \operatorname{Re}($ int $)=3$ calculation (solid lines) and the $\operatorname{Re}(b d y)=0.25, \operatorname{Re}($ int $)=8$ calculation (dashed lines). (b) shows results from the $\operatorname{Re}(\mathrm{bdy})=0.5, \operatorname{Re}($ int $)=3$ calculation (solid lines) and the $\operatorname{Re}(\mathrm{bdy})=0.25, \operatorname{Re}($ int $)=5$ calculation (dashed lines). 
$\operatorname{Re}($ int $)=8$ calculation has higher viscosity near the boundary and lower viscosity in the interior than the $\operatorname{Re}($ bdy $)=3, \operatorname{Re}(\mathrm{int})=3$ calculation. Thus, the gradients in the time-mean fields are weaker near the boundary and stronger in the interior in the $\operatorname{Re}(\mathrm{bdy})=0.25, \operatorname{Re}(\mathrm{int})=8$ case. This change in the gradients slightly widens the boundary current and moves it away from the boundary as can be seen in the $\beta$-term and mean advection term in figure 3-3a.

Along with the necessary change in the frictional flux convergence, the eddy, $\beta-$, and mean flux divergences are located farther away from the boundary in the $\operatorname{Re}(\mathrm{bdy})=0.25, \operatorname{Re}(\mathrm{int})=8$ calculation. Although I have not been able to pinpoint why each of these terms shifts outward, it should be clear that once the shift has occurred in the frictional fiux convergence, the rest must also shift if the structure of the time-mean fields is to be similar for the homoparic pair. The global structure of the time-mean fields is incredibly sensitive to the dynamics in this region (as pointed out in section 2.3.2). The same amount of vorticity must be transmitted across the same mean streamlines with the same efficiency, even if they are located slightly further away from the boundary. Therefore, as the frictional flux convergence shifts outward to allow for a reduced gradient when Re(bdy) decreases, the other terms in the vorticity equation must also occur farther out if the solution is to be homoparic.

As the members of a homoparic pair have the same input of vorticity by the wind and the same output of vorticity by friction (although with different vorticity gradients at the boundary), the pair member with higher Re(int) must have more efficient eddy transport of vorticity across mean streamlines in the interior. This must be the case as the time-mean vorticity field is similar to that of the other pair member, but the viscosity is smaller; thus, the implied frictional vorticity flux is smaller. In order to have the same transport of vorticity across mean streamlines, the eddy flux must be correspondingly larger. Figure 3-4 shows the ratio of the total vorticity flux to the purely frictional vorticity flux across mean streamlines. This reveals that indeed in the calculation with larger Re(int), the eddy flux across mean streamlines plays a 


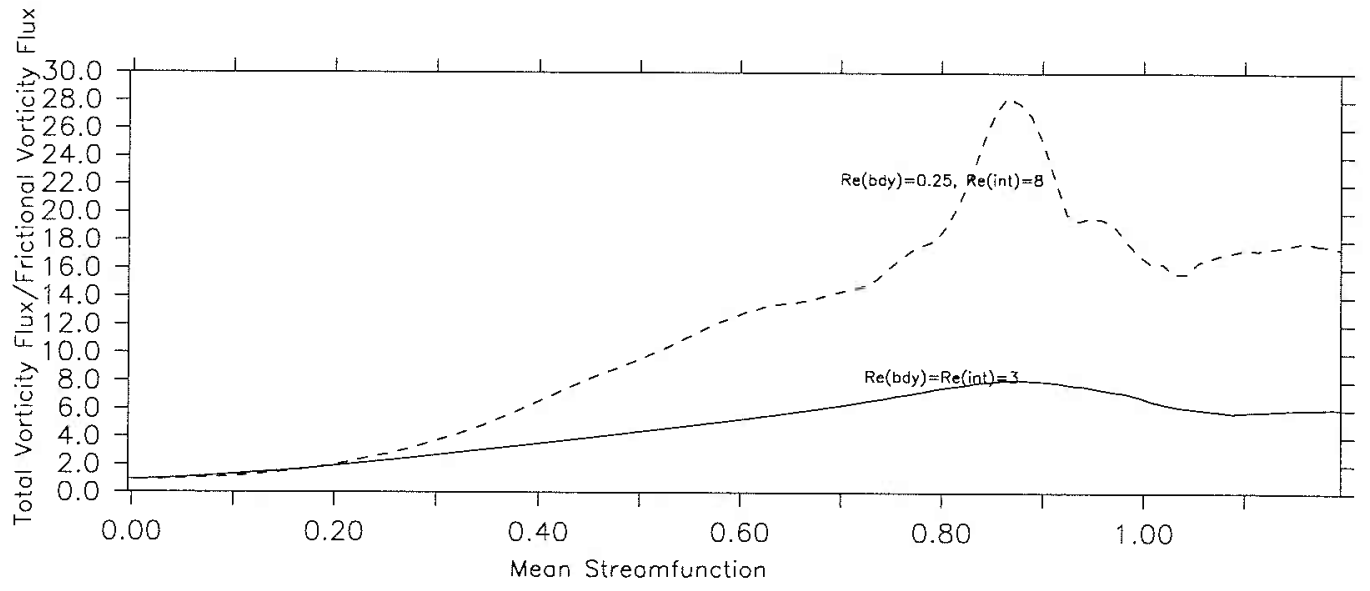

Figure 3-4: This figure shows the ratio of the total vorticity flux across a mean streamline to the frictional flux across a mean streamline as a function of mean streamfunction. It is constructed from the fluxes for the homoparic pair with $\operatorname{Re}($ int $)=\operatorname{Re}(b d y)=3$ and $\operatorname{Re}($ bdy $)=0.25, \operatorname{Re}($ int $)=8$.

larger role than the frictional flux across mean streamlines when compared to the other homoparic pair member.

The homoparic solutions in figure 2-2 also have similar eddy flux convergences (shown in figure 2-8). However, although the convergences are similar, the scale of the finer features in the convergence field is smaller in the pair member with larger $\operatorname{Re}($ int).

The smaller scale of eddies in the inviscid calculations can be easily understood as a change of viscosity in the interior of the basin strongly affects the enstrophy cascade there. In homoparic calculations, we expect that there will be more smallscale features in the vorticity field of the member of the pair with higher $\operatorname{Re}(\mathrm{int})$. It is pointed out in the introduction that considering eddy viscosity as merely the cutoff of enstrophy at high wavenumbers can be misleading when boundary layers are present. However, the cutoff of the enstrophy cascade remains a useful model for the dynamics of eddies' evolution away from the boundary. 
The presence of these small-scale enstrophic features can be seen by eye in figures $2-3$ and figure 2-8, but figure $3-5$ is a quantitative presentation. Figure 3-5 demonstrates that the amplitudes of small-scale Chebyshev modes tend to be larger in the homoparic pair member with a larger value of Re(int). Because the Chebyshev modes are smaller in scale at higher mode number, a slower decay in the spectrum with mode number indicates more small-scale phenomena. In this respect, the square of the Chebyshev coefficient is similar to a traditional squared-Fourier coefficient spectrum plotted by wavenumber, but with with enhanced weighting near the boundary. The connection between the Fourier and Chebyshev transforms is presented in Appendix A.

Finally, by careful analysis of figure 3-11, and by viewing of movies of the homoparic pairs, I have noted that there are significant differences in the spin-up time of the homoparic pair members. The one with greater Re(int) tends to take longer to spin-up. Thus, although the effects of the eddies are 'parameterized' by the changes in the viscosity, the parameterization does not extend fully to the time-dependence of the homoparic pairs.

In this section, I have shown that while the time-mean streamfunction of the homoparic pairs members is similar, upon evaluating the terms in the vorticity equation using these terms, small differences can be found. These small differences can be either in the magnitude of the frictional flux convergence and eddy flux divergence or in the gradients of all the terms. Because of the compensating changes, the resulting time-mean field is similar. It seems that there ought to be a way to analyze the mechanism of compensation closely and devise an effective eddy parameterization. I am unable to find a suitable approach to this problem, however promising it remains. In section 3.6, I return to the subject of homoparic pairs where it is shown these pairs of calculations differ energetically. In section 3.4, I also show that the homoparic pairs differ temporally. 

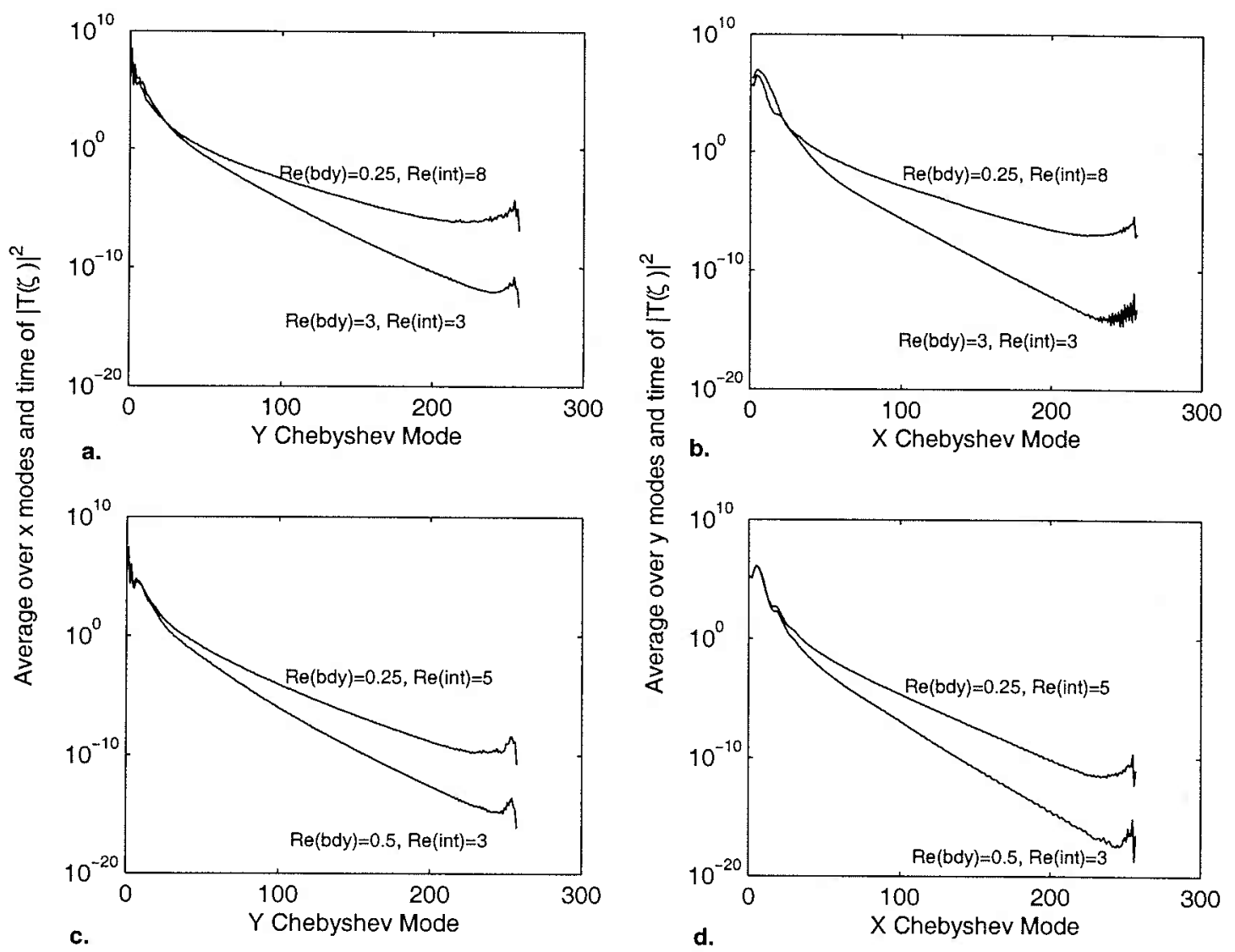

Figure 3-5: (a-d) show the spectral decay of the square of the Chebyshev coefficient of $\zeta$ two homoparic pairs. (a) shows the average over time and $x$-Chebyshev mode for the $\operatorname{Re}(\mathrm{bdy})=0.25, \operatorname{Re}($ int $)=8$ calculation and the $\operatorname{Re}(\mathrm{bdy})=3, \operatorname{Re}(\mathrm{int})=3$ calculation. (b) is the same as (a) except it shows the average over time and $y$-Chebyshev mode. (c) and (d) are the same as (a) and (b) except for the $\operatorname{Re}(b d y)=0.5, \operatorname{Re}($ int $)=3$ and $\operatorname{Re}(\mathrm{bdy})=0.25, \operatorname{Re}(\mathrm{int})=5$ homoparic pair.

\subsection{Counter-Rotating Gyres}

Preceding sections have focused on how eddies can take over for the friction flux across mean streamlines as the eddy viscosity is lowered. However, there are significant and important differences between the eddies and the eddy viscosity. One difference is the appearance of counter-rotating gyres in the more inviscid calculations.

Figure 2-2 reveals the presence of closed circulations in the southeast corner of the basin in the more inviscid calculations. The mean flow in these regions is in a direction 
opposite to that of the wind-stress; they are counter-rotating gyres. These counterrotating gyres are present in the figures of Holland and Lin (1975), Kamenkovich et al. (1995), and Sheremet et al. (1995), but are significantly weaker than those present here and go uncommented upon in the text of those papers.

Greatbatch and Nadiga (1999) and Holm and Nadiga (submitted 2002) observe these counter-rotating regions in double-gyre calculations with slip and superslip boundary conditions and in calculations with hyperviscosity instead of lateral friction. They also propose parameterization methods so that these counter-rotating regions will be present in coarse resolution calculations without eddies. In Greatbatch and Nadiga (1999), a parameterization of the effects of eddies is given in which the eddy parameterization fluxes vorticity down the mean gradient (equivalent to $\kappa \nabla\left(\delta_{I}^{2} \zeta+y\right)$ in my notation), but the boundary condition allows for no flux of absolute vorticity out of the basin. The solution which they obtain from this parameterization is steady, yet it possesses counter-rotating regions. This parameterization is not allowed in the models here, as there is a net vorticity input which could not be balanced were the boundary condition no flux of absolute vorticity. However, it is interesting to note that the parameterization used by Greatbatch and Nadiga (1999) produces counterrotating regions by simply homogenizing absolute vorticity. Thus, it is possible that the counter-rotating regions here may also be the effects of homogenization of absolute vorticity by the eddy field.

These gyres are not present in linear calculations (where the Sverdrup relation is satisfied in this region) or in steady-state solutions of the traditional nonlinear problem with lateral friction and slip or no-slip boundary conditions (see, for example Ierley and Sheremet (1995) and figures 1-1 and 1-2) because they are eddy-driven. By examining the region where $\bar{\psi}$ is negative in figure $2-9$ (which represents the counterrotating gyre in that calculation) one notes that friction does not significantly affect the cross-streamline vorticity flux in this region. All of the vorticity input within these mean streamlines is moved toward the boundary by eddies. During this process, the 
eddies and mean flow interact in such a way as to have an average circulation in a direction counter to the direction of the wind stress.

The eddy flux convergences in figure 2-8 and 2-6 reveal regions of eddy flux convergence in the region of the counter-rotating gyre (the closed contour filled with light coloring in the southeast quadrant of the less viscous calculations). The large spatial scale of these convergent regions indicates the character of these modes of vorticity transport. They are basin-scale motions reminiscent of the basin mode oscillations. The wave-like motion of absolute vorticity present in these modes can be seen propagating from east to west in the southern portion of figure $2-5$. In the more inviscid calculations, these modes have gained sufficient strength so as to drive a circulation, which is the counter-rotating gyre. These modes will be discussed further in section 3.5 .

Pedlosky (1965b) solves for the weakly-nonlinear interaction of basin modes forced by a traveling wave $\left(w_{E}=\cos (k x-\omega t) \sin (n \pi y)\right)$ in a homogeneous model with bottom friction only. He finds that in the weakly nonlinear limit, the zeroth-order solution responds linearly to the wind forcing while the first-order solution contains a response to the nonlinear interaction of the zeroth-order solution. This result is relevant here as the zero-frequency response to the nonlinear interaction can possess a region which rotates counterclockwise in the southern portion of the basin. However, Pedlosky (1965b) proposes a primary balance for this region with the eddy flux convergence balanced by the $\beta$-term, whereas the counter-rotating regions here are in primary balance between the eddy flux convergence and the steady wind forcing with the $\beta$-term as a next-order correction (see figure 3-6). Regardless, the results of Pedlosky (1965b) show that a nonlinear interaction of basin modes can cause a steady circulation, and I believe that a similar analytic calculation can be created to explain the counter-rotating regions here.

To explore the possibility that these counter-rotating regions are driven by basin modes, a simple additional experiment was performed. A calculation was performed 

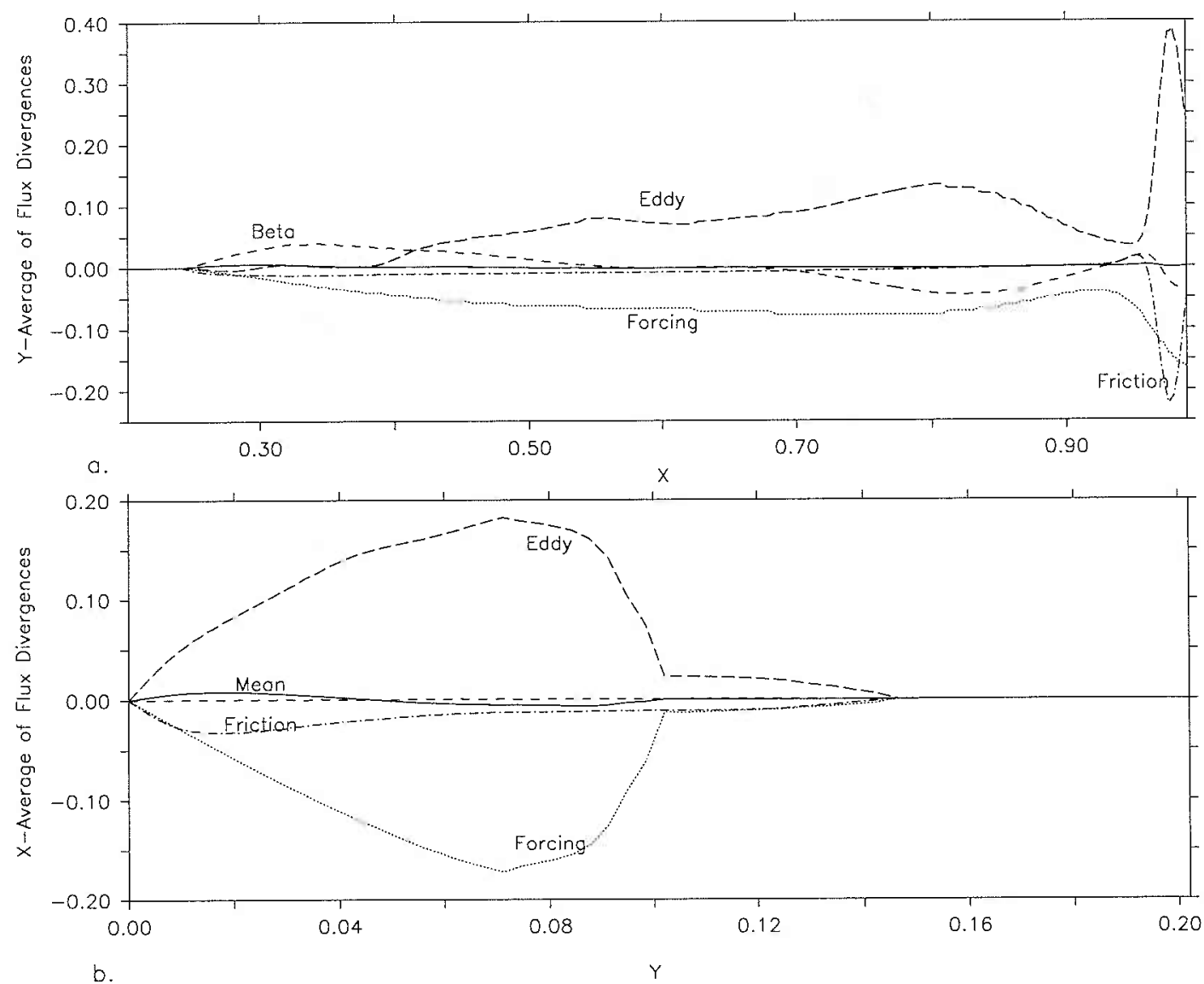

Figure 3-6: (a) and (b) show the meridional and zonal averages, respectively, of terms in the time-mean vorticity equation in the region where $\bar{\psi}<0$ (the counter-rotating gyre) from the $\operatorname{Re}(\mathrm{bdy})=0.25, \operatorname{Re}($ int $)=5$ calculation.

with a basin that was extended to be twice as long in the zonal direction, except with wind forcing only in the western half of the basin. Therefore, the linear Munk solution would only extend halfway across the basin. Different calculations using this model are presented in section 3.5, and more discussion on the model is presented there. Figure 3-7 shows the time-mean streamfunction from this calculation. There are clearly steady circulations which occur in the eastern half of the basin where there is no wind forcing. In fact, there is a region which rotates counterclockwise in the southern half of the basin as the counter-rotating gyres do, and there is a region 


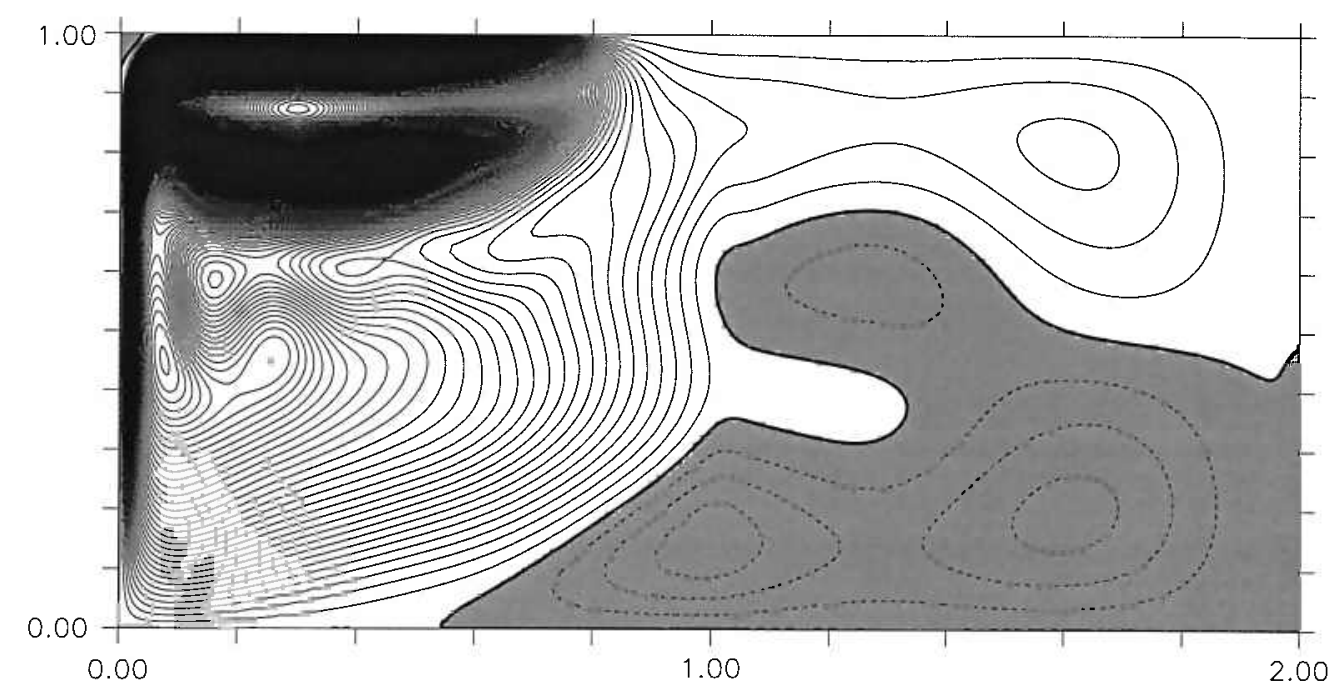

Figure 3-7: The time-mean streamfunction showing the the counter-rotating gyre in a calculation in a elongated basin with $\operatorname{Re}($ bdy $)=3, \operatorname{Re}($ int $)=3$. There is wind forcing only in the western half of the basin. The contour interval is 0.025 . regions of negative streamfunction are shaded.

which rotates clockwise in the northern half of the basin. This is just as in some of the analytic solutions of Pedlosky (1965b).

Furthermore, figure 3-8 shows the meridional and zonal averages of the terms in the vorticity equation in the counter-rotating region. The most southwestern part of the counter-rotating gyre extends into the wind forcing, and therefore experiences some wind forcing, which is balanced by the eddy flux convergence in the zonal mean (figure 3-8b). However, in figure 3-8a, it becomes clear that only the westernmost part of the counter-rotating region experiences the wind forcing and the easternmost region is frictional. The middle of the counter-rotating region has a balance between the $\beta$-term and the eddy flux divergence, just as the resonant solutions in Pedlosky (1965b) do. Therefore, it seems that in the regions devoid of the wind forcing, a 

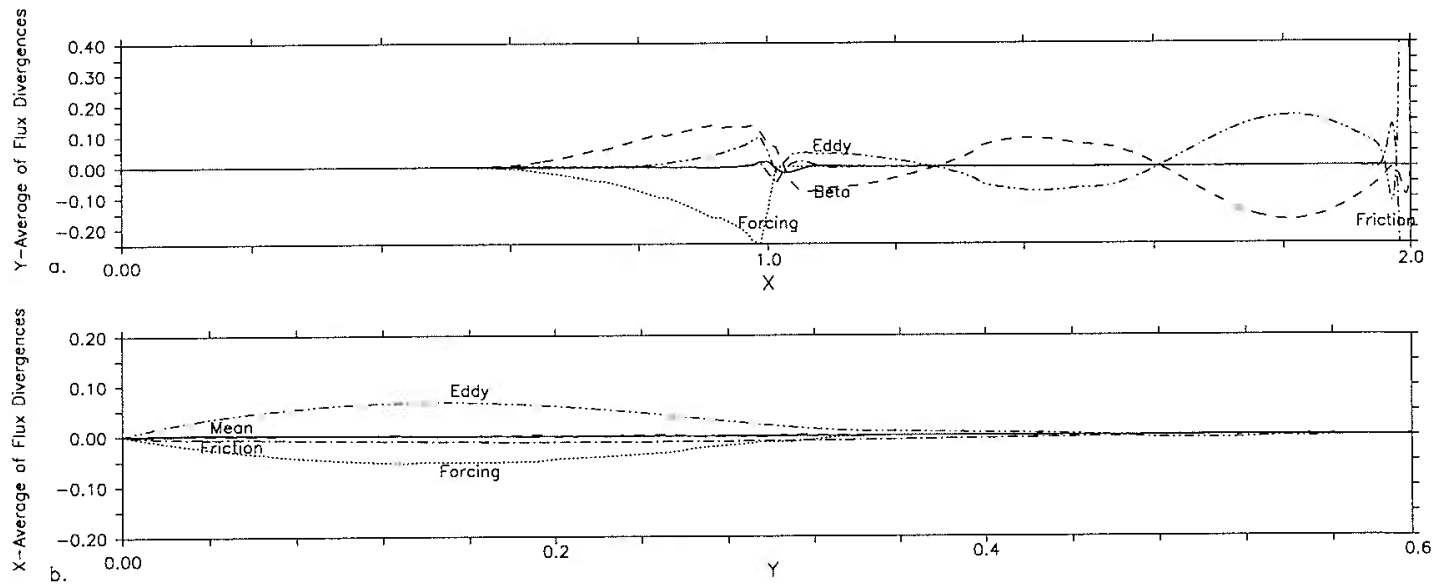

Figure 3-8: (a) and (b) show the meridional and zonal averages, respectively, of terms in the time-mean vorticity equation in the region where $\bar{\psi}<0$ (the counter-rotating gyre) from the long basin calculation in figure 3-7.

version of the dynamics proposed by Pedlosky (1965b) with lateral friction would apply. Also, so long as the western boundary region is excluded, it appears that only a small change would be needed to add in the effects of a steady wind forcing.

I have used empirical orthogonal functions to determine the structure and frequencies of the large scale modes in the calculation shown in figures 3-7 and 3-8. ${ }^{1}$ The result is that the large scale motions in this model are consistent with the presence of modes of variability similar to one of the basin modes. The nonlinearly-forced first-order solution given by Pedlosky (1965b) for this basin mode has three equallyspaced counter-clockwise gyres in the southern half of the basin and three equallyspaced clockwise gyres in the northern half of the basin (figure 3-9). The pattern of eddy-driven regions in figure 3-7 is consistent with such a pattern. It is quite easy to imagine three gyres above and below the midpoint of the basin. Considering that Pedlosky's solutions use bottom friction and possess no mean flow resulting from steady wind-forcing, this result is highly suggestive that the proper explanation of

\footnotetext{
${ }^{1}$ These results are not shown, but more will be said about using empirical orthogonal functions to identify basin modes in sections 3.4 and 3.5 . The mode found here is the $(m, n)=(3,1)$ mode.
} 


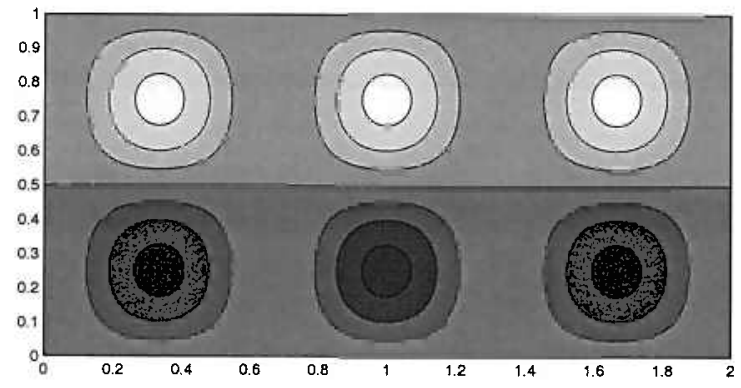

Figure 3-9: Contours of the streamfunction of the analytic first-order estimate by Pedlosky (1965b) of the steady circulation resulting from the nonlinear interaction of the $(3,1)$ basin mode. Light colors are clockwise circulations, dark colors are counterclockwise circulations.

these counter-rotating gyres is to be given by an analysis similar to that of Pedlosky (1965b).

Returning to the case where there is wind forcing throughout the basin, if these basin modes are global and forced at the opposite end of the basin, how is it that the adjustment occurs so that the wind forcing is exactly balanced by the eddies? There seems to be a causal problem in this case. Of course, it is not the eddies which adjust, but the mean flow; if the closed streamlines contain too much or too little wind stress then they will build up a vorticity anomaly and can not remain in that position at equilibrium.

It is interesting that the counter-rotating gyres exist, because in this region of the basin, the Sverdrup solution would be adequate. Yet, it should be quite clear from the inertially-dominated solutions that the adequacy of the Sverdrup solution is not sufficient to guarantee that effects from other parts of the basin do not overrun the Sverdrup solution. If the hypothesis that the basin modes interact nonlinearly to create these regions, then a forcing in a remote region excites the basin modes (which are addressed in section 3.5) which in turn cause a circulation in this region in a sense opposite to that expected from the local wind forcing.

The counter-rotating regions play an intriguing role in the energy budget, as the 
energy input of the wind to these regions is negative. For example, in the $\operatorname{Re}(b d y)=3$, $\operatorname{Re}($ int $)=7$ calculation, the counter-rotating gyre reduces the energy input to the mean flow by $12.6 \%$ when compared to the same solution except with $\psi$ set to zero in the counter-rotating region. The necessity of reduction of energy input as Reynolds number is increased has been proposed previously by Scott and Straub (1998). The energetic impact of the counter-rotating gyre is discussed in section 3.6 .

Salmon et al. (1976) propose that the most likely equilibrium statistical mechanical solution to the quasigeostrophic equations is the one which maximizes entropy production. Griffa and Salmon (1989) were able to show that in a closed basin the maximal entropy production states for the unforced, non-dissipative calculation are modes resembling those of Fofonoff (1954). These Fofonoff-like modes have been seen in models more recently (for example, Griffa and Castellari (1991), Özgökmen and Chassignet (1998)). One might suspect that the counter-rotating gyres seen here are of this variety. However, this is not the case, as the Fofonoff (1954) modes are primarily a balance between mean advection of vorticity and the $\beta$-term and are symmetric in the zonal direction (as discussed in the introduction). Fofonoff-like solutions are also characterized by inertial boundary currents on the eastern and western coasts. Furthermore, the ansatz of the Fofonoff solution-that $q=q(\psi)$ is not satisfied in the counter-rotating region (see figure 3-10).

Figure 3-6 shows the meridional and zonal averages of the terms in the vorticity equation within the counter-rotating region. This figure reveals that the counterrotating gyres have a strong asymmetry from east to west and the primary balance of terms in the vorticity equation is wind forcing balanced by eddy flux convergence. The unforced, non-dissipative limit sought by Griffa and Salmon (1989) is not applicable here. Only as higher-order corrections to this primary balance do the mean advection and $\beta$-term enter.

Interestingly, the other correction at the next order is a frictional sublayer on the eastern boundary, driven by the eddy flux convergence. As this gyre is counter- 


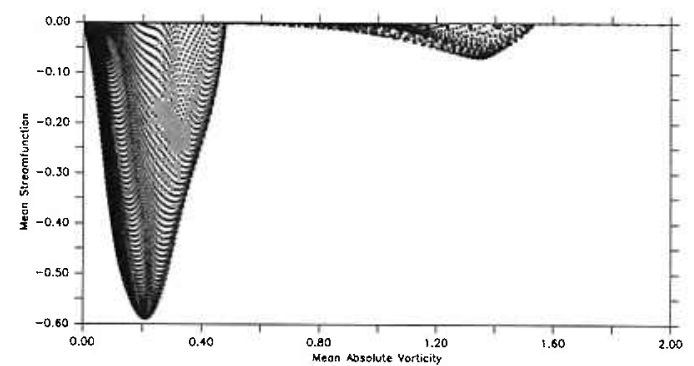

Figure 3-10: The relationship between the mean values of $\delta_{\bar{I}}^{2} \bar{\zeta}+y$ and $\bar{\psi}$ from the $\operatorname{Re}(\mathrm{bdy})=5, \operatorname{Re}(\mathrm{int})=5$ calculation. Note that the relationship is not a single-valued function as required by the Fofonoff ansatz.

rotating, the frictional sublayer acts in the opposite sense from elsewhere in the basin. The friction fluxes vorticity of the same sign as the wind input into the basin in the counter-rotating region. Figure 3-6 shows the terms in the vorticity equation which are important in these gyres.

The counter-rotating gyres are therefore quite unlike the Fofonoff-like modes described by Griffa and Salmon (1989), at least for the case of no-slip boundary conditions at the eastern and western boundaries. However, the question of whether the counter-rotating gyre represents the maximal entropy production solution remains open but is outside the scope of this investigation.

To summarize, the strong basin-mode like oscillations in the relatively inviscid calculations drive a region of the mean flow which rotates in a direction opposite to the wind forcing. The primary balance of terms in these regions is eddy flux convergence and wind forcing. The counter-rotating regions appear to be effects of the nonlinear interactions of basin modes similar to those studied by Pedlosky (1965b). The dynamics are remarkably similar when a basin with wind forcing in only the eastern half of the domain is used. The counter-rotating regions are unlike the Fofonoff (1954) solution and the Griffa and Salmon (1989) statistical mechanics solutions for freely-evolving flow. These counter-rotating regions reduce the wind 
power input, as will be discussed further in section 3.6. These counter-rotating regions are proof that although eddies sometimes neatly replace the action of eddy viscosity in the basin interior, at other times they behave quite differently, even producing non-local effects.

\subsection{Temporal Structure of the Solutions}

Resolved eddies differ from eddy viscosity in the temporal character of the solution. In a linear calculation, where eddy viscosity represents all of the effects of the eddies, a steady wind forcing always results in a steady solution. Even if the wind forcing is variable, the frequencies of the response by a linear system are easily predicted by a knowledge of the resonant modes of the system. In the inviscid calculations presented here, despite the steady forcing the solution has a significant range of variability on many time scales, both resonant and not.

Even within the small parameter range studied here, there is a diversity of temporal behaviors. As shown in figure 2-4, there is a qualitative difference in the parametric response between solutions that have a recirculation gyre which reaches the eastern boundary (inertially-dominated) and those that do not (western-intensified). There is also a qualitative difference in temporal behavior. The western-intensified solutions can be further divided into three categories: steady solutions, harmonic solutions, and chaotic solutions. All four cases are shown in figure 3-11.

The lowest Reynolds number calculation shown in figure 3-11 has $\operatorname{Re}(b d y)=0.5$ and $\operatorname{Re}(\mathrm{int})=0.5$, is western-intensified and reaches a steady state. This solution is only slightly influenced by nonlinear terms, and its response to forcing is much like that of a linear model.

At slightly higher Reynolds number, the solutions remain western-intensified, but the kinetic energy is seen to oscillate periodically or exhibit a superposition of a few frequencies. This is the regime where instabilities are present, but only of a 


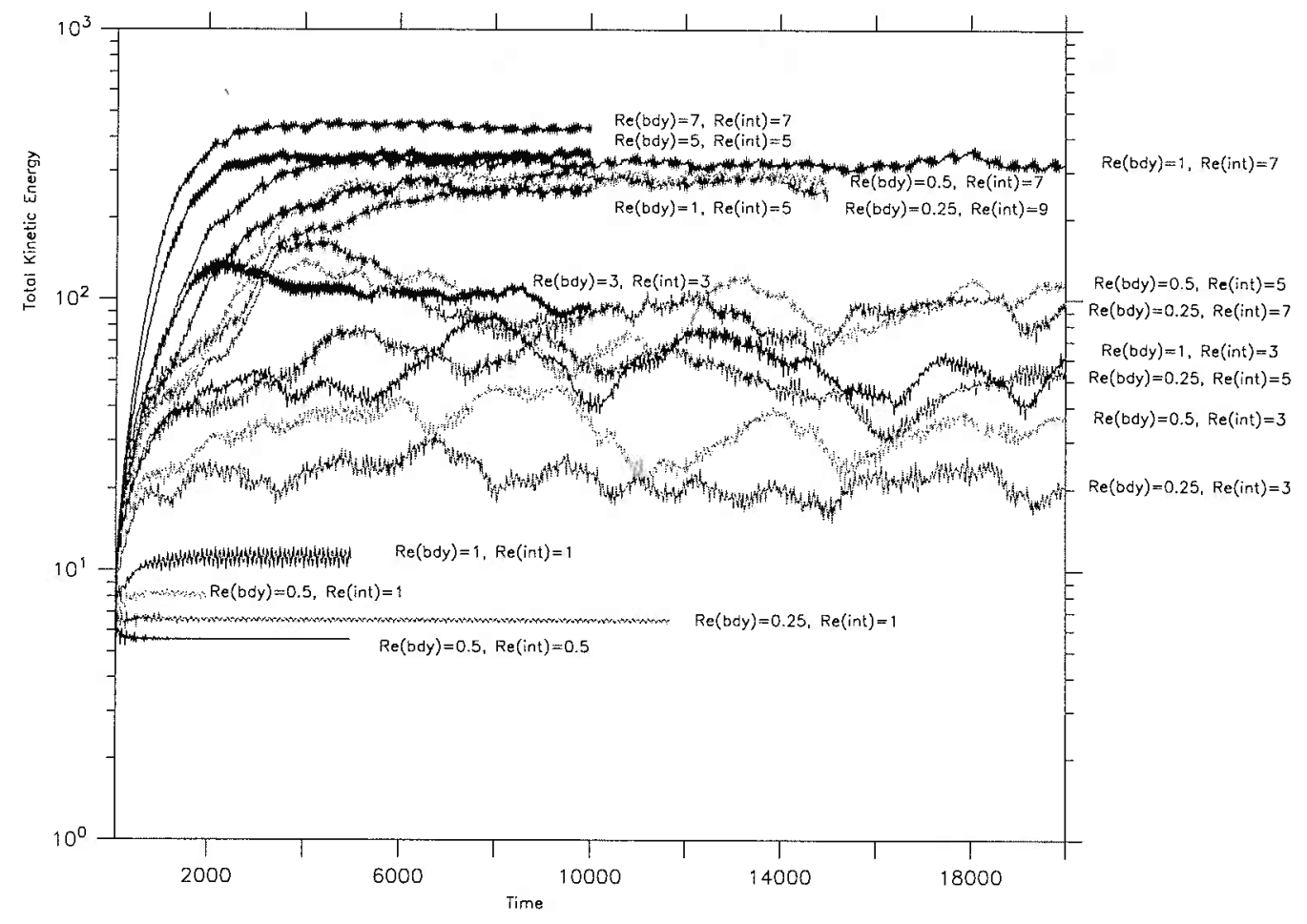

Figure 3-11: Semi-log plot of total kinetic energy $\left(\iint \psi^{2} \mathrm{dA} / 2\right)$ versus time evaluated every 25 time units for different calculations. The four temporal behavior classes are exemplified here (ordered from small total kinetic energy to large) steady, harmonic solutions, and chaotic solutions which are western-intensified and chaotic solutions which are inertially-dominated. The averaging interval for the time-means given throughout this dissertation is usually the second half of the length of integration. 
few types. Each type has a typical frequency, and the variability caused by these instabilities interacts in a weakly nonlinear manner to produce harmonics. Because these modes are weak and do not strongly affect the mean flow, these calculations oscillate about a mean solution which is very similar to the steady solution. The modes of instability present in these calculations are addressed in Sheremet et al. (1997), where an analysis of the eigenfunctions of linear perturbations about the steady-state solutions is presented. This analysis is able to correctly predict the onset of instability. However, Sheremet et al. (1997) do not fully address the nonlinear interaction between these modes, so mention of it will be made here.

As an analytic example to place the nonlinear interaction in context, consider a weakly-nonlinear perturbation analysis of two basin mode oscillations occurring simultaneously. If the modes are free and inviscid, to lowest order they must satisfy the non-dimensional equation

$$
\frac{\partial \nabla^{2} \psi}{\partial t}+\frac{\partial \psi}{\partial x}=0
$$

The streamfunction must also be constant along the boundary to guarantee impermeability of the boundaries. If, as in the numerical model, the boundaries are located where $x$ and $y$ are zero and one, then the solutions for mode number $(m, n)$ are of the form

$$
\begin{gathered}
\psi=\Re\left[\psi_{0} \sin (m \pi x) \sin (n \pi y) e^{i\left(\sigma_{m n} t+\frac{x}{2 \sigma_{m n}}\right)}\right] \\
\psi \equiv \Re\left[E_{m n} e^{i\left(\sigma_{m n} t+\frac{x}{2 \sigma_{m n}}\right)}\right], \\
\sigma_{m n}=\frac{-1}{2 \pi \sqrt{m^{2}+n^{2}}} .
\end{gathered}
$$

For a more complete introduction to the free basin modes, see Pedlosky (1987).

The complex function $E_{m n}$ is the envelope function for mode $(\mathrm{m}, \mathrm{n})$ and is complex so that it sets the phase of the wave as well.

In a series expansion of the weakly nonlinear perturbation analysis, the next order 
in the expansion will be forced by the evaluation of the nonlinear interaction of these linear, lowest-order modes. As an example, consider a weak nonlinear interaction between only two basin modes, the $(m, n)$ mode and the $(k, l)$ mode. Evaluating one of the two nonlinear terms in the vorticity equation (\#1) gives

$$
\begin{array}{rc}
\# 1= & J\left(\psi(k, l), \nabla^{2} \psi(m, n)\right) \\
= & J\left(\psi(k, l), \Re\left\{\left[-2\left(m^{2} \pi^{2}+n^{2} \pi^{2}\right)+\frac{i}{2 \sigma_{m n}}\right] E_{m n} e^{i\left(\sigma_{m n} t+\frac{x}{2 \sigma_{m n}}\right)}\right\}\right) \\
= & \Re\left\{\left[\left(\frac{\partial E_{k l}}{\partial x}+\frac{i E_{k l}}{2 \sigma_{k l}}\right) \frac{\partial E_{m n}}{\partial y}-\frac{\partial E_{k l}}{\partial y}\left(\frac{\partial E_{m n}}{\partial x}+\frac{i E_{m n}}{2 \sigma_{m n}}\right)\right] e^{i\left[\left(\sigma_{k l}+\sigma m n\right) t+\frac{x\left(\sigma_{k l}+\sigma m n\right)}{2 \sigma_{k l} \sigma_{m n}}\right]}\right\} \\
& +\Re\left\{\left[\left(\frac{\partial E_{k l}^{*}}{\partial x}-\frac{i E_{k l}^{*}}{2 \sigma_{k l}}\right) \frac{\partial E_{m n}}{\partial y}-\frac{\partial E_{k l}^{*}}{\partial y}\left(\frac{\partial E_{m n}}{\partial x}+\frac{i E_{m n}}{2 \sigma_{m n}}\right)\right] e^{i\left[\left(-\sigma_{k l}+\sigma m n\right) t+\frac{x\left(\sigma_{k l}-\sigma m n\right)}{2 \sigma_{k l} \sigma_{m n}}\right]}\right\} .
\end{array}
$$

The other nonlinear term, $J\left(\psi(m, n), \nabla^{2} \psi(k, l)\right)$, will be similar in form, except with $(\mathrm{k}, \mathrm{l})$ and $(\mathrm{m}, \mathrm{n})$ exchanged. This calculation shows that the frequency of the interaction term-and also by symmetry the frequency of the other interaction term $\left(J\left(\psi(m, n), \nabla^{2} \psi(k, l)\right)\right)$-depend on the sum and the difference of the frequencies of the original modes. Therefore the frequencies of the variability forced by this nonlinear interaction will also be at or near frequencies which are the sums and differences of the original frequencies.

This is the reason why the nonlinear interactions between basin modes as treated by Pedlosky (1965b) has relevance for the counter-rotating gyres. If there are two modes of different phase with the same $(m, n)$, then their nonlinear interaction will force a response at two frequencies: $\sigma=0$ and $2 \sigma_{m n}$. It is the steady response which is to be considered as a cause for the counter-rotating regions.

Notice also that the wavenumbers of the interaction terms are the sums and differences of the wavenumbers of the original modes:

$$
\begin{aligned}
& \frac{\left(\sigma_{k l}-\sigma_{m n}\right)}{\sigma_{k l} \sigma_{m n}}=\frac{1}{\sigma_{k l}}-\frac{1}{\sigma_{m n}} \\
& \frac{\left(\sigma_{k l}+\sigma_{m n}\right)}{\sigma_{k l} \sigma_{m n}}=\frac{1}{\sigma_{k l}}+\frac{1}{\sigma_{m n}} .
\end{aligned}
$$


Most of the interaction here is between modes which are not pure waves and so do not have an obvious wavenumber, but this result is still useful is interpreting the spatial structure of the interaction modes.

Although the free, linear basin modes are not exactly the eigenfunctions solved for by Sheremet et al. (1997), they are similar in their interaction. Therefore, in the weakly nonlinear limit, we expect to see a small number of unstable modes, which interact nonlinearly with frequencies that are the sum and difference of the modes' frequencies. Figure 3-12 shows just such an interaction between two periodic modes in the $\operatorname{Re}(\mathrm{bdy})=1, \operatorname{Re}(\mathrm{int})=1$ calculation.

In figure 3-12, the empirical orthogonal functions (EOFs) were calculated from the relative vorticity field. The presence of these EOFs over time was then calculated. Approximately two EOFs are present for each mode of instability.

In chapters 3 and 4 , EOFs are calculated in an attempt to empirically quantify the primary modes of variability in the time-dependent numerical model. The empirical orthogonal functions are discussed in detail elsewhere (for example, Berkooz et al. (1993), Wunsch (1996)). In these calculations, the quantity of interest $(\psi, \zeta$, etc.) was interpolated onto a uniformly-spaced $101 \times 101$ grid for a lengthy subsection $(0(3000)$ units of $1 /(\beta L))$ of the integration time after equilibration had occurred. The empirical orthogonal functions were then calculated on this grid over this time interval.

The EOFs are roughly able to represent basin modes, but not exactly. The basin modes have the form given in equation 3.3. With appropriate definitions of the complex coefficients $\psi_{1}$ and $\psi_{2}$, this can be rewritten as a sum of two standing waves:

$$
\begin{aligned}
\psi= & \Re\left\{\psi_{1}\left[\sin (m \pi x) \sin (n \pi y) \sin \left(\frac{x}{2 \sigma_{m n}}\right)\right] e^{i \sigma_{m n} t}\right\} \\
& +\Re\left\{\psi_{2}\left[\sin (m \pi x) \sin (n \pi y) \cos \left(\frac{x}{2 \sigma_{m n}}\right)\right] e^{i \sigma_{m n} t}\right\}
\end{aligned}
$$

The spatial pattern of these standing waves (in brackets) does not change as the phase 

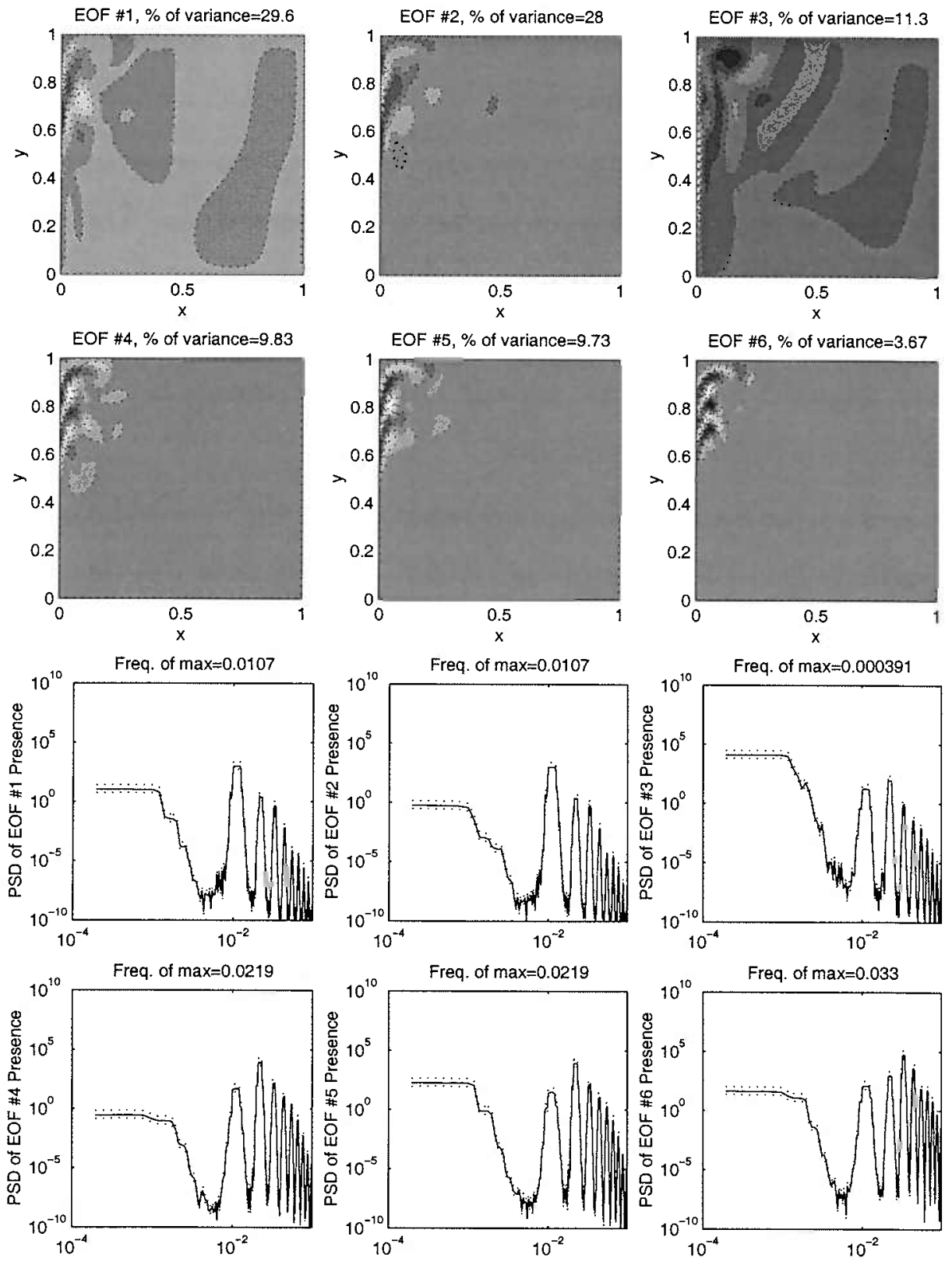

Figure 3-12: The upper two rows contour the EOFs of relative vorticity perturbation from the time-mean for the first six EOFs for the $\operatorname{Re}(b d y)=1, \operatorname{Re}($ int $)=1$ calculation and give the percent of the total variance associated with each EOF. The lower two rows give a multi-taper estimate of the power spectral density of the presence of each of the six EOFs. The $95 \%$ confidence interval is shown with dotted lines. 

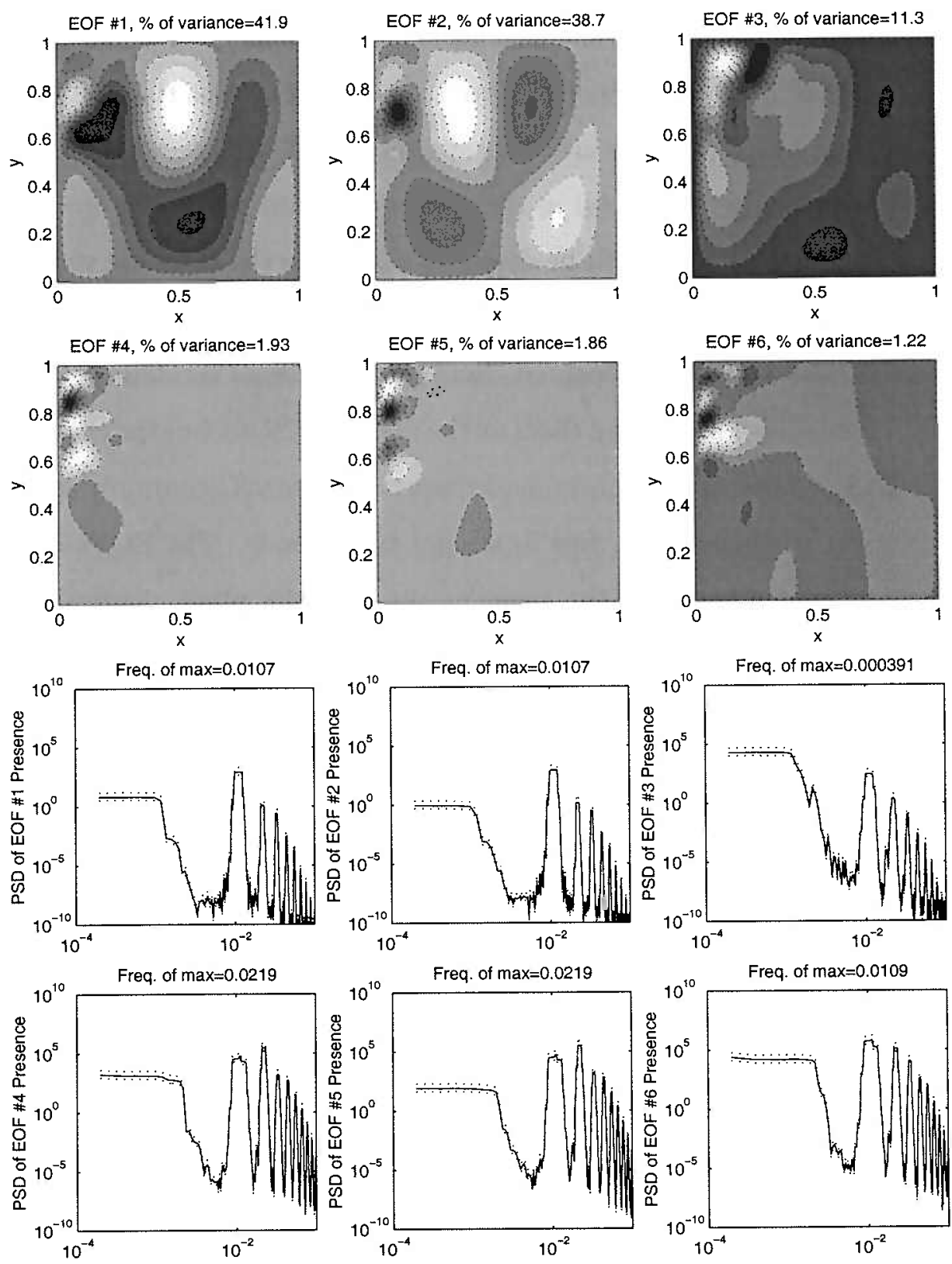

Figure 3-13: As in figure 3-12 except for the EOFs of streamfunction perturbation from the time-mean for the $\operatorname{Re}(b d y)=1, \operatorname{Re}($ int $)=1$ calculation. 
of the wave changes, instead the their magnitude increases and decreases with time. The decomposition into the two standing waves is not unique, a constant could have been added to the spatial phase of each (e.g., $\left.\cos \left(\frac{x}{2 \sigma_{m n}}+\Delta\right)\right)$. The two EOFs which were generated per basin mode should agree to some extent with the spatial structure of these standing waves (at some arbitrary spatial phase). However, as these standing waves are not spatially orthogonal in the $\mathrm{x}$-direction, and the EOFs are required to be spatially orthogonal, there will be differences between the standing waves and the EOFs. These differences will increase with increasing zonal wavenumber as the basin modes become less and less orthogonal. Simple experiments combining a number of linear basin modes and processing them as the numerical model output was processed revealed that low-wavenumber basin modes were reproduced accurately in space and with the correct frequency with two EOFs per basin mode. The EOFs strongly resembled the standing waves of the basin modes, but the phase determining which standing-wave patterns are produced is arbitrary. As the wavenumber increased (especially the zonal wavenumber) the correspondence trailed off as edge effects became important. These issues are discussed in more detail elsewhere (e.g., Joliffe (1986) and Wunsch (1997)).

Along with the spatial pattern of the EOFs, the presence of each EOF in time was calculated. A power spectrum density estimate (PSD) was made from the time series of the presence of each EOF. The peaks in this PSD should correspond to the dominant frequencies that occur as each EOF increases and diminishes. The PSDs shown in figure 3-12 possess a number of obvious peaks. ${ }^{2}$

One of the peaks corresponds to the frequency of a boundary-current instability. This mode of instability has a signature in the EOFs of both streamfunction and relative vorticity and is contained in a region near the boundary (see figures 3-12 and

\footnotetext{
${ }^{2}$ It has come to my attention that the MATLAB routine I used to calculate these modes has an error in the determination of confidence intervals. Therefore, the confidence intervals shown here should be considered to be approximate only to within a factor of 2 . Additionally, because the confidence interval assumes Gaussian, white noise errors, whereas this process is deterministic and non-white, there may be other problems with the confidence limits.
} 
3-13, EOFs number 4 and 5). The frequency of this boundary-current instability is $0.0219 \beta L .^{3}$

A second peak that appears in the PSDs of two EOFs shown in figures 3-12 and 3-13 seems to correspond to the $(1,2)$ basin mode. This peak occurs at $0.0107 \beta L$. The $(1,2)$ linear basin mode in this basin has a frequency of $0.0113 \beta L$ (figure 3-14), and the patterns of the streamfunction in figure 3-13 strongly resemble the basin mode standing waves in figure 3-14, at least in the region outside of the boundary current. The EOFs of relative vorticity with this frequency do not resemble the relative vorticity pattern for a basin mode. Possibly, the basin mode coincides in frequency with activity in the boundary current which would explain the shape of EOFs 1 and 2 of relative vorticity perturbation in figure 3-12. The similar frequency of the boundary-trapped relative vorticity perturbation EOFs and the basin-modelike EOFs suggests that there is a boundary current instability which resonates with the basin mode. The next section will further address the role and presence of basin modes in these solutions.

The peak at $0.033 \beta L$ in figure 3-12 presumably represents variability forced by the nonlinear interaction of the basin mode-like variability (or the boundary current instability forcing it) and the boundary current instability, as the sum of $0.0219 \beta L$ and $0.0107 \beta L$ is $0.0326 \beta L$. The other large peaks in the PSD are located at the sums and differences (and their sums and differences) of the two original frequencies to within the resolution of the PSD. Furthermore, the EOF corresponding to the peak at $0.033 \beta L$ has a smaller spatial scale than the EOFs of relative vorticity of the $0.0219 \beta L$ mode and the and $0.0107 \beta L$ mode. It is entirely plausible that the wavenumber of the $0.033 \beta L$ mode is the sum of the wavenumbers of these two modes as it should were this EOF corresponding to a nonlinear interaction mode.

All of the EOFs have some signal at all of the frequencies mentioned. This could be reflective of the involvement of each EOF in nonlinear interaction at each of the

\footnotetext{
${ }^{3}$ All of the frequencies derived from EOFs are calculated as simply the frequency at the maximum of the PSD.
} 

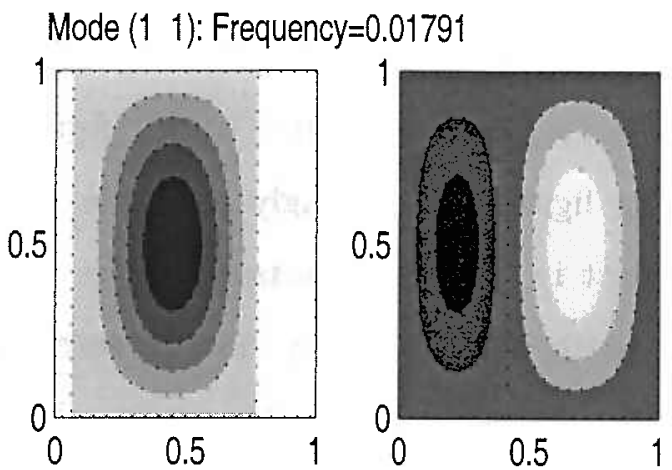

Mode (1 2): Frequency $=0.01133$
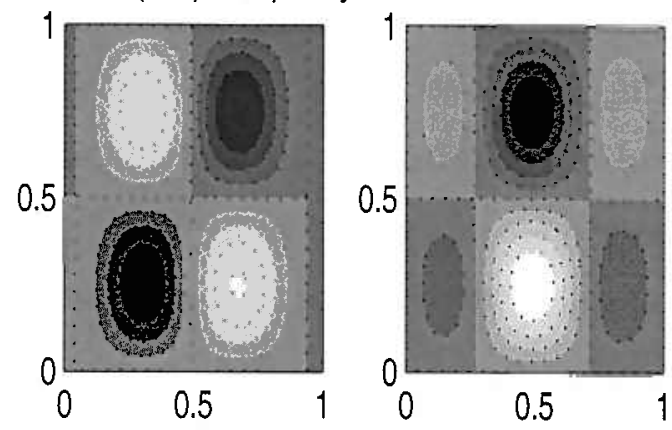

Mode (1 1): Frequency $=0.02266$
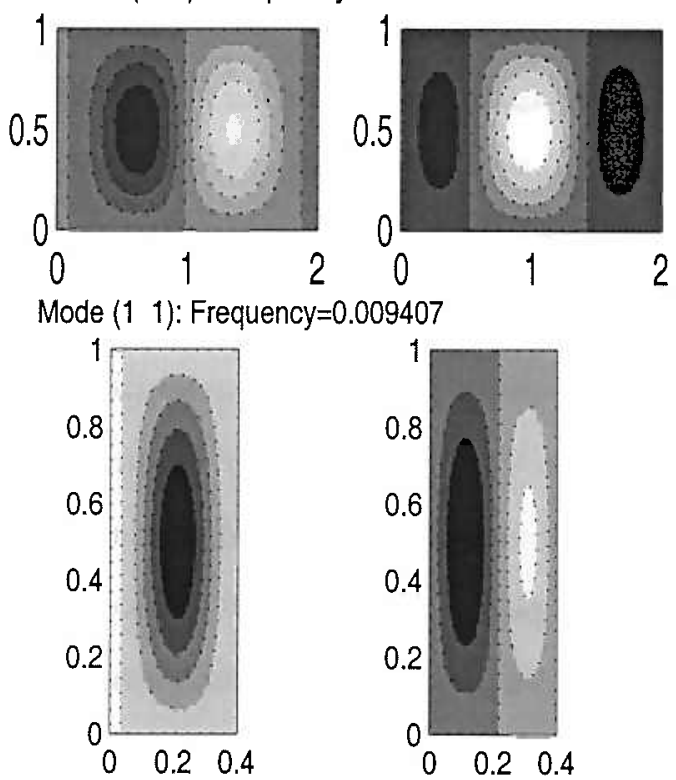

Mode (3 2): Frequency $=0.01013$
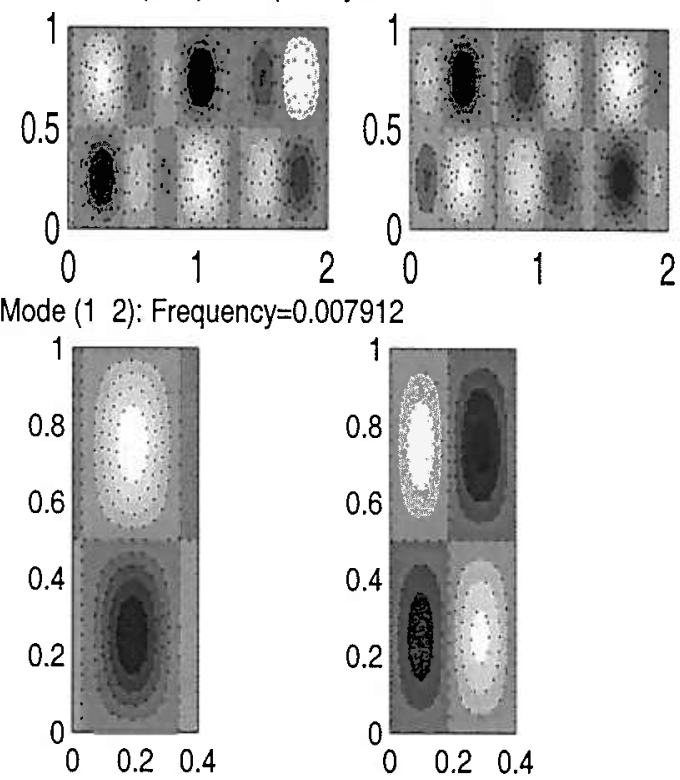

Figure 3-14: The upper row contours the two standing-wave streamfunction patterns (with an arbitrary choice of phase) for two basin modes in a square basin. The middle row contours the two standing-wave streamfunction patterns for two basin modes in a $1 \times 2$ basin. The lower row contours the two standing-wave streamfunction patterns for two basin modes in a $1 \times 0.4$ basin. The frequencies (in $\beta L$ units, true frequencies not angular frequencies) and mode number of each mode are also given. 
frequencies, but it could also be an artifact of the errors in producing the EOFs. Because of the lack of orthogonality of the modes which lead to each EOF, it is expected that there be some leakage between the frequencies of the different modes. This was observed in the hypothetical test case described above of the EOF decomposition of a linear superposition of noninteracting basin modes. Herein, I assume that the largest magnitude peak in the spectral decomposition corresponds to the primary frequency of the mode involved.

The strong activity in the northwest corner of the basin in all of the EOFs is the region where the linear instabilities described by Sheremet et al. (1997) originate, and apparently this is the case here as well. In the following section, it will be shown that even in a different-sized basin, the activity in this region is what forces many of the motions observed.

One other main conclusion of Sheremet et al. (1997) was the presence of a resonant mode of instability. They called this mode resonant because its frequency was comparable to that of the basin modes in their calculation and so they expected that they would resonate with the basin modes. This mode appears to be present here as EOF number 3 in figures 3-12 and 3-13.

At higher Reynolds number, the relative vorticity EOFs, although still westernintensified, become temporally chaotic (figure 3-11). ${ }^{4}$ Upon calculating the EOFs (figure 3-15) there are some periodic structures revealed, although they are not clearly distinct as in the lower Reynolds number cases. Also, although the total variance has increased, the percentage of the variance associated with any one EOF has decreased. Essentially, at this point the solution is quite turbulent, and there are many modes of instability and nonlinear interaction present. There do not appear to be any lowfrequency modes per se, but due to the white spectrum of all of the EOFs at low frequency, low frequency variability is expected and is present.

However, when the EOFs of the streamfunction perturbation are examined (see

\footnotetext{
${ }^{4}$ Here I employ the term chaotic because the time series of chaotic systems are are typically non-repeating and aperiodic as is this time series. No other definition of chaos is implied.
} 

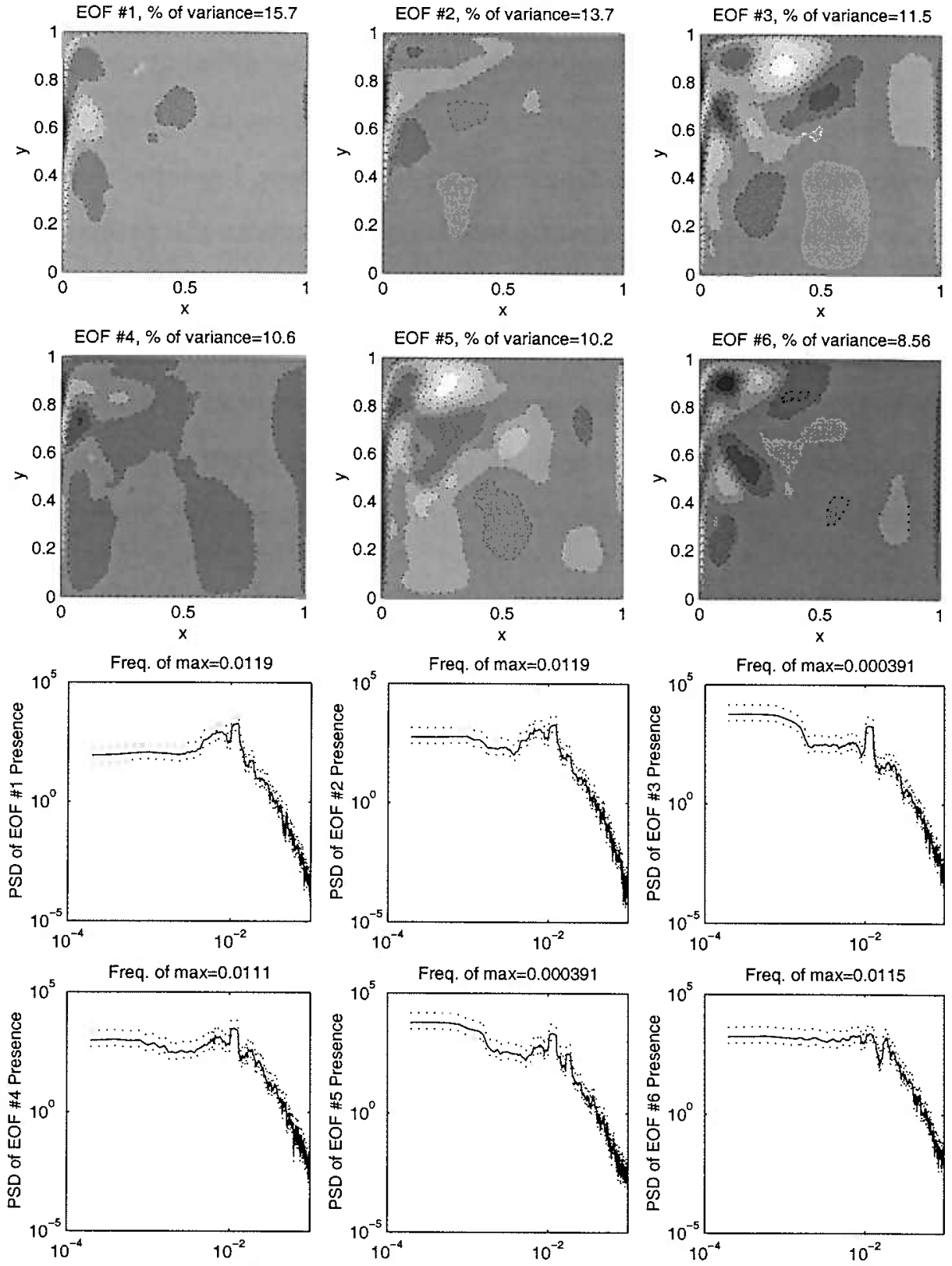

Figure 3-15: The EOFs of relative vorticity perturbation from the time-mean as in figure $3-12$ but for the $\operatorname{Re}(\mathrm{bdy})=0.25, \operatorname{Re}(\mathrm{int})=5$ calculation which shows large lowfrequency variations in figure $3-11$. 
figure 3-16), a different picture emerges. Although the variability of the relative vorticity had a broadband spectrum, there are still two modes of streamfunction variability which are obviously periodic. These modes of instability strongly resemble the gravest basin mode and the $(1,2)$ basin mode standing wave patterns, and the frequencies of these the linear modes agree well with the observed frequencies of the variability (see figure 3-14). These modes will be discussed further in the next section.

In the calculations where the recirculation gyre has reached the eastern boundary, the temporal behavior remains complicated, but the range of variability is decreased. As shown in figure 2-4a, the ratio of eddy energy to mean flow energy is still quite large, but the amount of each stabilizes once the recirculation gyre reaches the eastern boundary. The EOFs of an inertially-dominated solution are shown in figure 3-17.

In summary, the temporal behavior of the single-gyre calculation is unsteady and complicated, in stark contrast to the steady solutions to the wind-driven problem, even with linear Rossby waves superposed. There are numerous modes of instability of the mean state, and these modes both force the basin modes to oscillate and interact nonlinearly with each other. These interactions lead to a broadband spectrum for many of the EOFs and for the total kinetic energy in calculations with high Re(int). As part of the broadband spectrum, there are low-frequency motions which in the oceanographic context may be significant for climate variability, a topic of contemporary scientific interest. No low frequency pure oscillations were observed.

\subsection{Presence of Basin Modes}

Many of the preceding sections have mentioned the presence of variability that resembles the free basin modes of the homogeneous fluid model. Because these modes are only present in a closed basin, it is important to assess whether they are present here, why, and what effects they have on the mean flow because many studies of the western boundary current instabilities use regional models lacking these basin modes. 

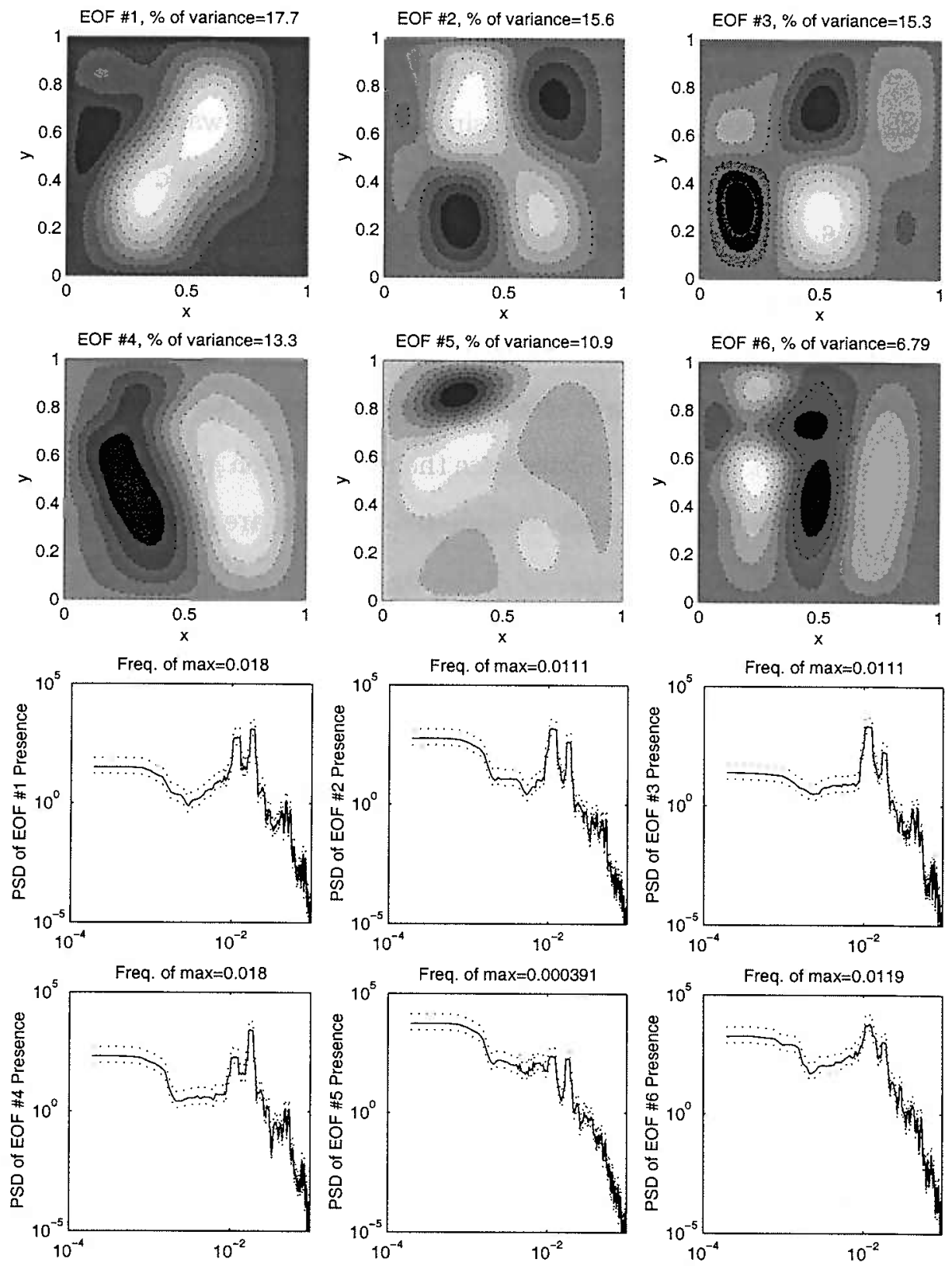

Figure 3-16: EOFs of streamfunction perturbation from the time-mean as in figure 313 but for the $\operatorname{Re}($ bdy $)=0.25, \operatorname{Re}($ int $)=5$ calculation which shows large low-frequency variations in figure $3-11$. 

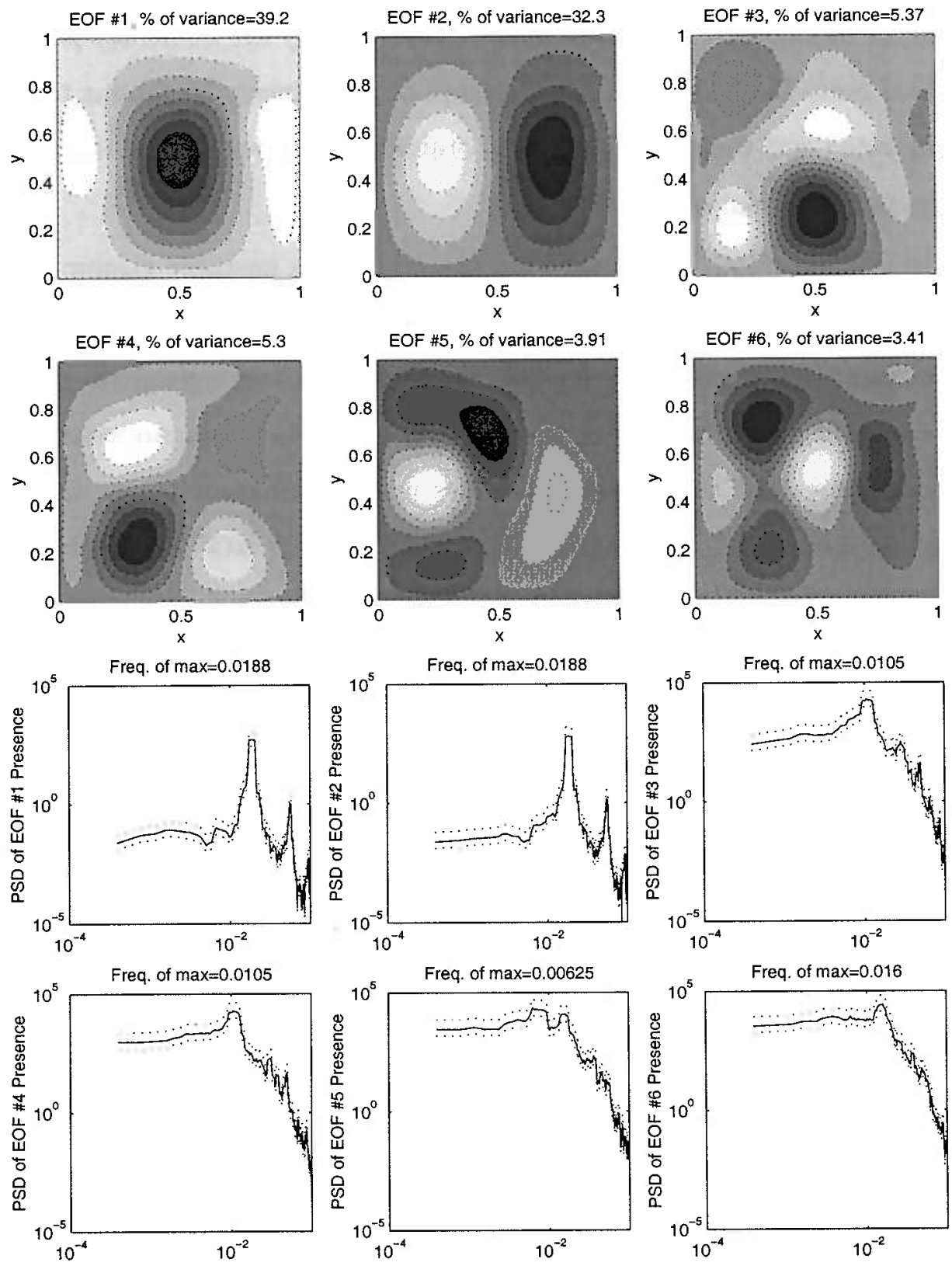

Figure 3-17: EOFs of streamfunction perturbation from the time-mean as in figure 313 but for the $\operatorname{Re}($ bdy $)=5, \operatorname{Re}($ int $)=5$ calculation . 
Since the premise of boundary-enhanced viscosity is that there are boundary processes too fine to resolve in a basin-wide model, it is particularly important to show that regional models can be used in future work to study the boundary processes parameterized here. In this section, the presence of phenomena resembling these modes is demonstrated and their effects on the instability of the western boundary currents and the general circulation are discussed.

Sheremet et al. (1997) demonstrated that there are unstable eigenmodes in the problem of linear perturbations of the steady-state solution to the single-gyre with the same boundary conditions presented here. Some of these eigenmodes, which they dubbed resonant modes had frequencies comparable to the basin modes of oscillation. They hypothesize that these resonant modes would resonate with the basin modes. As we saw in section 3.4, some of the EOFs resembled the EOFs of basin modes and the resonant mode.

To test whether these modes that resemble basin modes are actually related to the basin modes of oscillation, a simple test was performed. The model was run with basins of different sizes for the same viscosity and boundary current transport. The first basin was the standard square basin that has been used thus far. The second basin was elongated in the zonal direction to double the width. In the elongated basin case, the wind forcing was set to zero for the eastern half of the model. The third basin was shortened in the zonal direction to $40 \%$ of the width. In the shortened basin case, the wind forcing was increased by a factor of 3.1 in magnitude. ${ }^{5}$ In this way, the western boundary current in the new basins is as close to the square basin as possible. Exact reproduction of the conditions in the western boundary current is not possible, but the remaining differences are minor. Only the effects of changing the eastern frictional boundary current and changing the wind forcing magnitude in the shortened basin's boundary current remain. Therefore, the primary effect of moving

\footnotetext{
${ }^{5}$ This empirical factor that sets the strength of the wind forcing was chosen to attempt match the boundary current transport. If the Sverdrup balance held over the whole basin and the boundary layer was infinitesimally thin the constant would be 2.5 .
} 


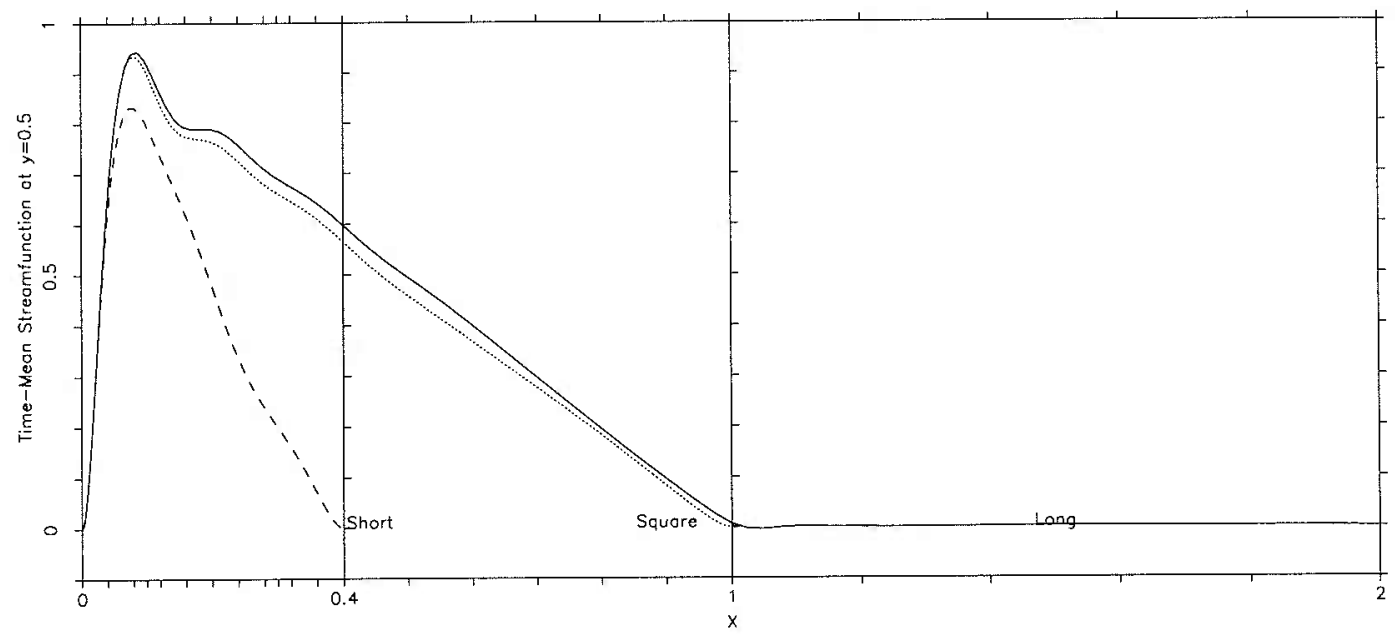

Figure 3-18: The time-mean streamfunction at $y=0.5$ for the short basin, the square basin, and the long basin with $\operatorname{Re}($ int $)=\operatorname{Re}($ bdy $)=1$.

the eastern boundary is the change in the reflection of waves (and basin modes) at the eastern boundary. The resulting mean streamfunction at the north-south midpoint for the calculations in the three different basins is shown in figure 3-18.

The results for the EOFs of the perturbation streamfunction from the calculations in the differently-sized basins are shown in figures 3-13, 3-19, and 3-21. The results for the relative vorticity perturbation EOFs are given in figures 3-12, 3-20, and 322. It is clear that the basin mode-like oscillations in the longer basin have a higher wavenumber and a different frequency, while there are no obvious modes present in the short basin case.

The basin mode-like motions observed in figures 3-13 and 3-19 can be compared in shape and frequency to the linear basin modes; some of the linear modes were chosen for comparison and are shown in figure 3-14. As pointed out in the last section, EOF number 1 and 2 of figure 3-13 (the square basin) strongly resemble the $(1,2)$ linear basin mode. In the longer basin, figure 3-19 shows that the streamfunction perturbation EOFs number 1 and 2 resemble the $(3,2)$ basin mode in standing-wave 

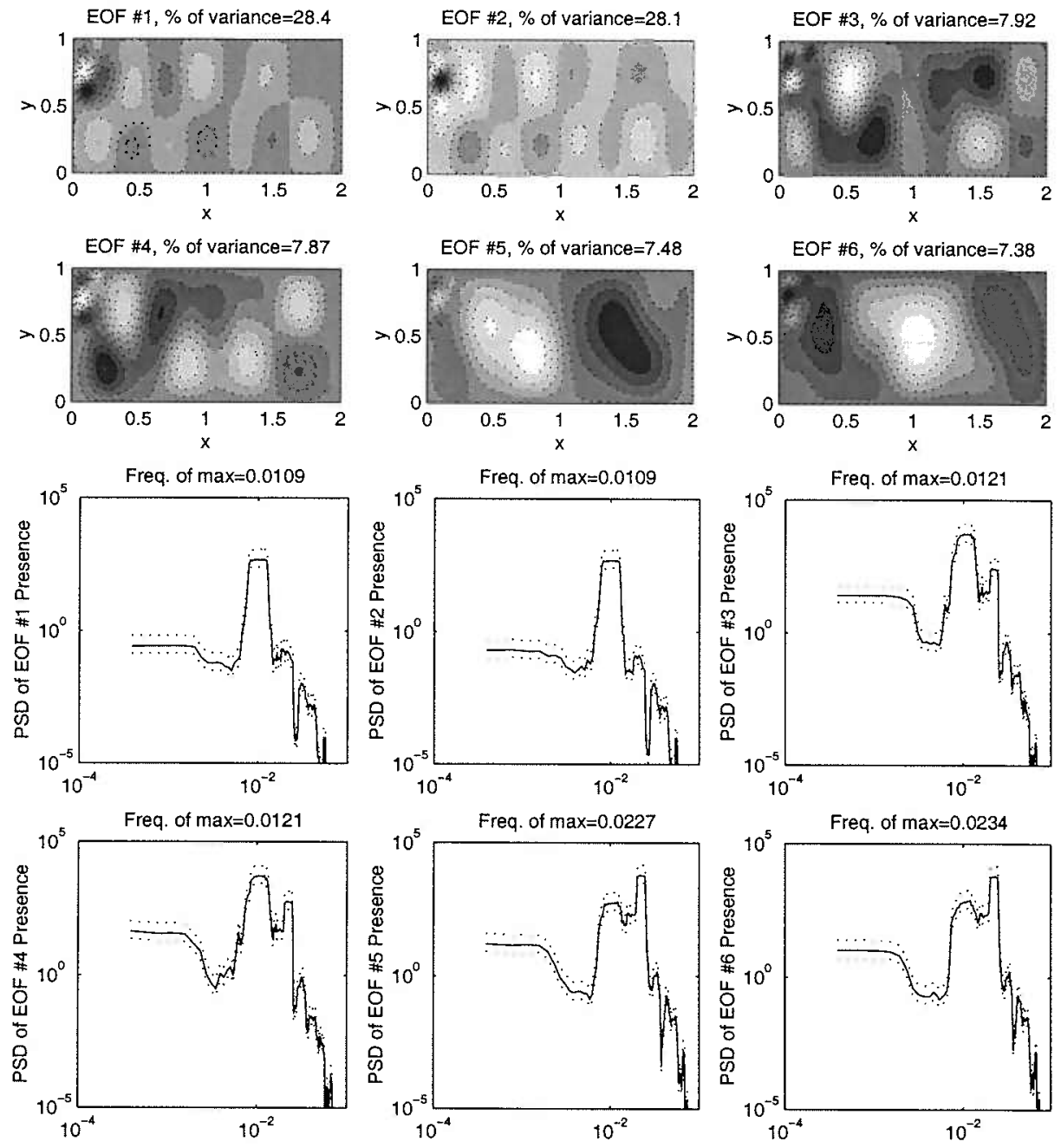

Figure 3-19: EOFs of streamfunction perturbation from the time-mean as in figure 313 but for the $\operatorname{Re}(\mathrm{bdy})=1, \operatorname{Re}(\mathrm{int})=1$ calculation in a long basin of twice the zonal extent. 

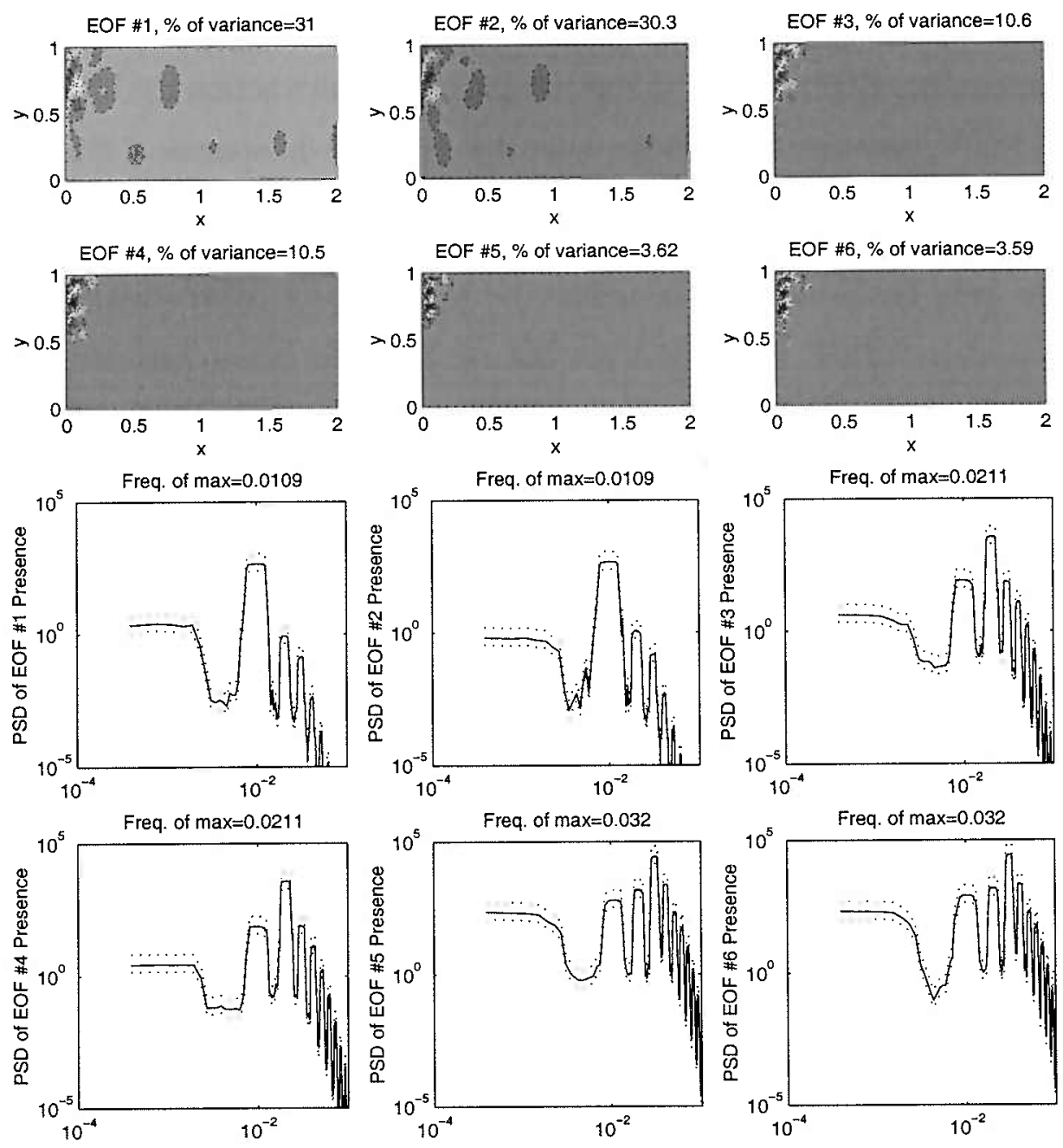

Figure 3-20: EOFs of relative vorticity perturbation from the time-mean as in figure 312 but for the $\operatorname{Re}($ bdy $)=1, \operatorname{Re}($ int $)=1$ calculation in a long basin.

pattern and frequency while EOFs number 5 and 6 resemble the gravest basin mode in standing wave frequency and pattern. Also, EOFs number 3 and 4 (with frequency $0.0121 \beta L$ ) are roughly similar to the $(1,2)$ basin mode's standing wave patterns which have a frequency of $0.0123 \beta L$.

Figure 3-20 shows the EOFs of relative vorticity for the long basin. Comparing with figure $3-12$, it is apparent that there are similar frequency EOFs in the relative vorticity perturbations. These EOFs have most of their variability in a region closer 
to the boundary than the EOF patterns of the streamfunction perturbation. Again, the lower variance EOFs appear to represent nonlinear interaction modes between the previous EOFs, because the frequencies are the sums and differences of the frequencies of the higher variance EOFs.

In the long basin calculation, unlike the square basin calculation, some of the relative vorticity's EOF frequencies are distinct from the streamfunction's EOF frequencies. They share a similar frequency range, however. Since the streamfunction EOFs' frequencies closely correspond to the basin mode frequencies, I propose that the relative vorticity EOFs represent the instabilities that are forcing the basin modes. If this is correct, then we can see that the frequencies of the instabilities are relatively unchanged by the change of basin from square to long (0.0107 to $0.0109 \beta L$ and 0.0219 to $0.0211 \beta L$ ). However, which basin modes are excited by these modes of instability is dependent on which basin modes share frequencies with these instabilities. Note especially that the nonlinear interaction mode at represented by EOF 6 in figure 320 has no corresponding large variance streamfunction EOF in figure 3-19. This is precisely as one would expect given that there are no basin modes with a frequency this high.

Why is it that the streamfunction EOFs resemble the basin modes while the relative vorticity EOFs seem to represent the boundary layer phenomena? Of course, it is important to remember that the EOF is an ex post facto analysis which has no information about the underlying dynamics. The goal of the EOF is simply to present a set of orthogonal functions, beginning with the one responsible for the maximum percentage of the variance to the one responsible for the least. In these models, typically the magnitude of the streamfunction is not significantly greater in the boundary current while the relative vorticity is much greater in the boundary current (see figure 2-5). Thus, in considering the modes which will have the greatest variance the large scale of the basin modes will be dominant since the streamfunction has smaller point-by-point excursions. However, in considering the relative vorticity, 
small changes in the location of the extremely large values of relative vorticity that are present near the boundary seem to cause greater variance than the large-scale, but weaker magnitude basin modes.

So, comparing the long basin to the square basin, it seems that changing the basin dimension without significantly changing the forcing seems to leave the boundary current variability unchanged to within measurable limits. However, different basin modes are excited with different amplitudes because their resonant frequencies change relative to that of the basin. In the square basin, the $(1,2)$ basin mode was excited. Thus, the relationship between the boundary current instabilities and the basin modes strongly affects which modes are excited.

Figure 3-14 shows that the frequencies of the free basin modes are reduced in the shortened basin. The maximum frequency is that of the gravest mode; in the shortened basin it is $0.0094 \beta L$. All of the other basin modes have lower frequencies. Since the western boundary current instabilities have been carefully preserved when the eastern boundary is moved, their frequencies should be relatively unchanged. in figures 3-12 and 3-20, the lowest frequency western boundary instability peak appeared at $0.0107 \beta L$. Thus, we expect that in figure 3-21 the frequencies of the disturbances in the western boundary current should be higher than all of the basin modes' frequencies. We therefore expect that no basin modes will be excited.

Examining the frequencies of the peaks that are present in the shortened basin, all are higher in frequency than the gravest mode frequency $(0.0094 \beta L)$. These frequencies are slightly higher (about 15\%) than the frequncies in the square basin and the long basin. Apparently, either the lack of the basin modes has changed the boundary current instabilities (unlikely), the EOFs are misleading ${ }^{6}$, or the boundary current instabilities are affected by the change in the basins.

I believe it is most likely that the setup of the short basin is not perfect. Initially, I performed the calculation in a short basin with a wind forcing of the appropriate

\footnotetext{
${ }^{6}$ This is highly unlikely as the same frequencies are found in the PSD of vorticity at points in the boundary layer.
} 

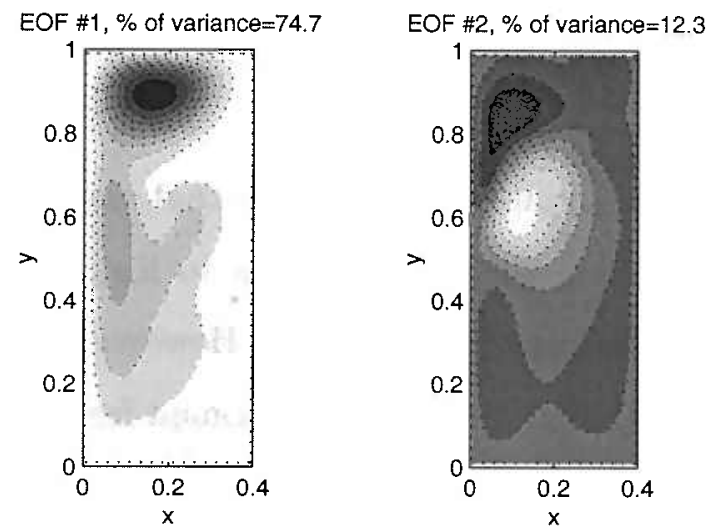

EOF \#4, \% of variance $=0.937$ EOF \#5, \% of variance $=0.905$
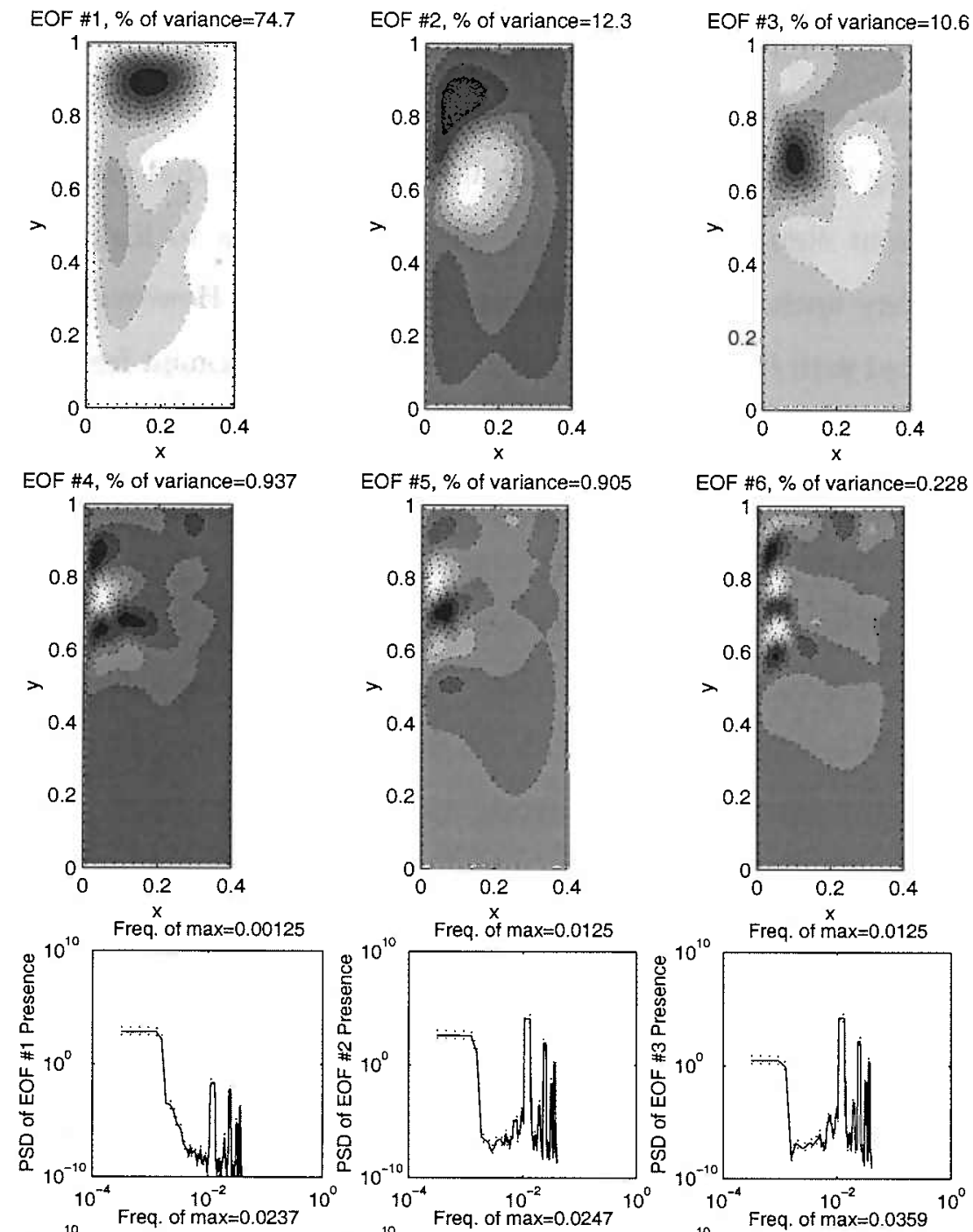

EOF $\# 6, \%$ of variance $=0.228$
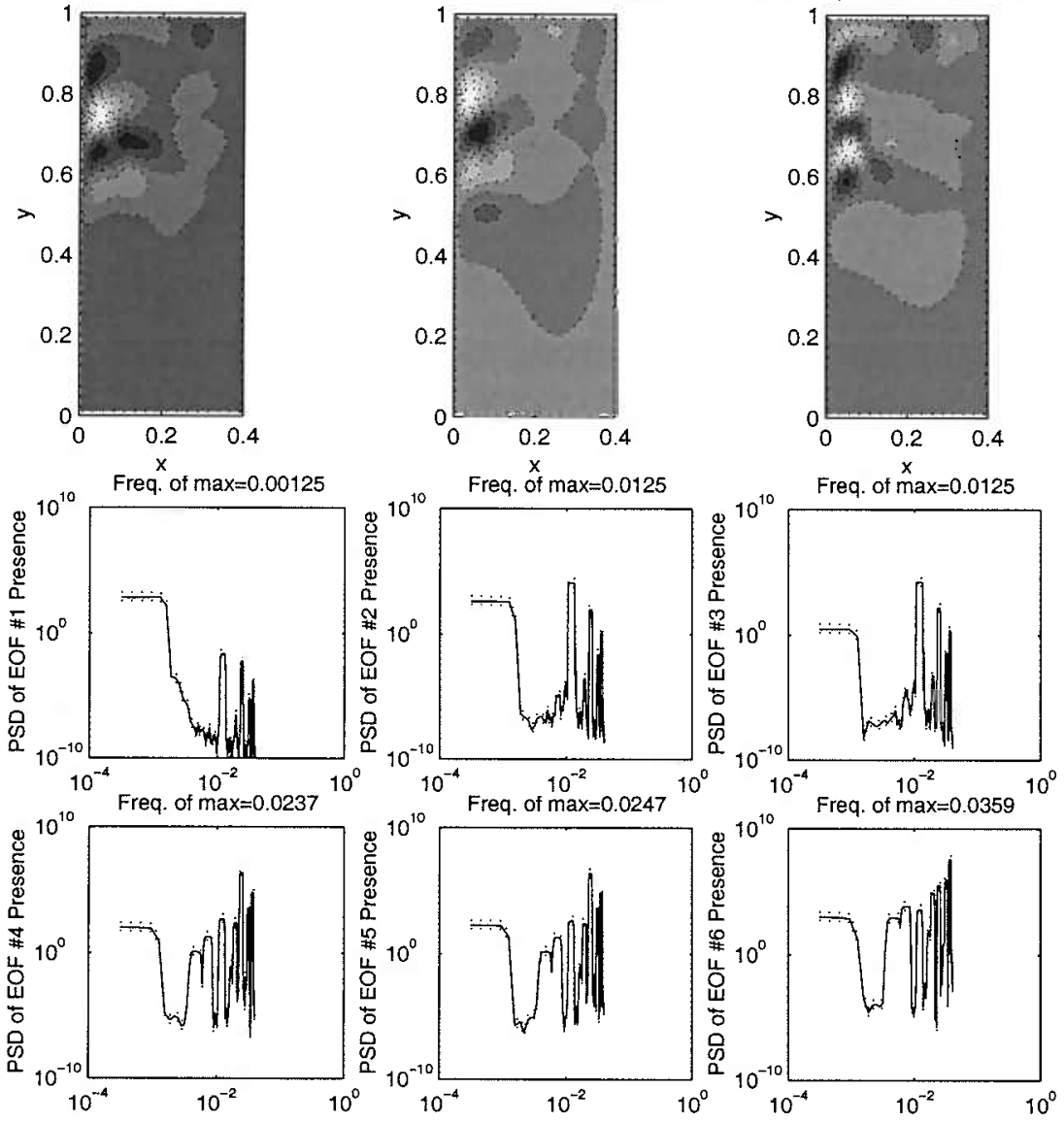

Figure 3-21: EOFs of streamfunction perturbation from the time-mean as in figure 313 but for the $\operatorname{Re}(\mathrm{bdy})=1, \operatorname{Re}(\mathrm{int})=1$ calculation in a short basin of zonal extent 0.4 . 

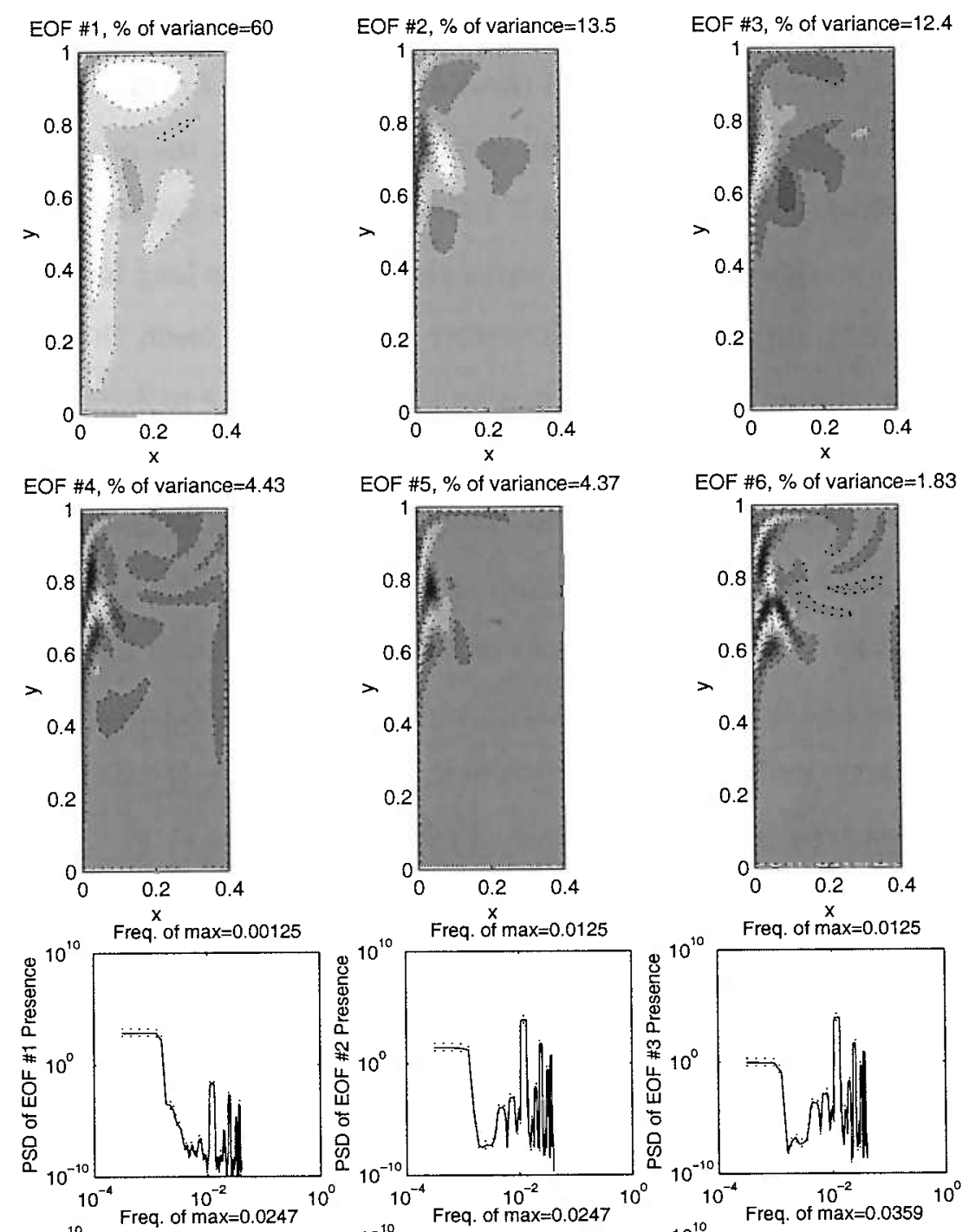

EOF $\# 6, \%$ of variance $=1.83$
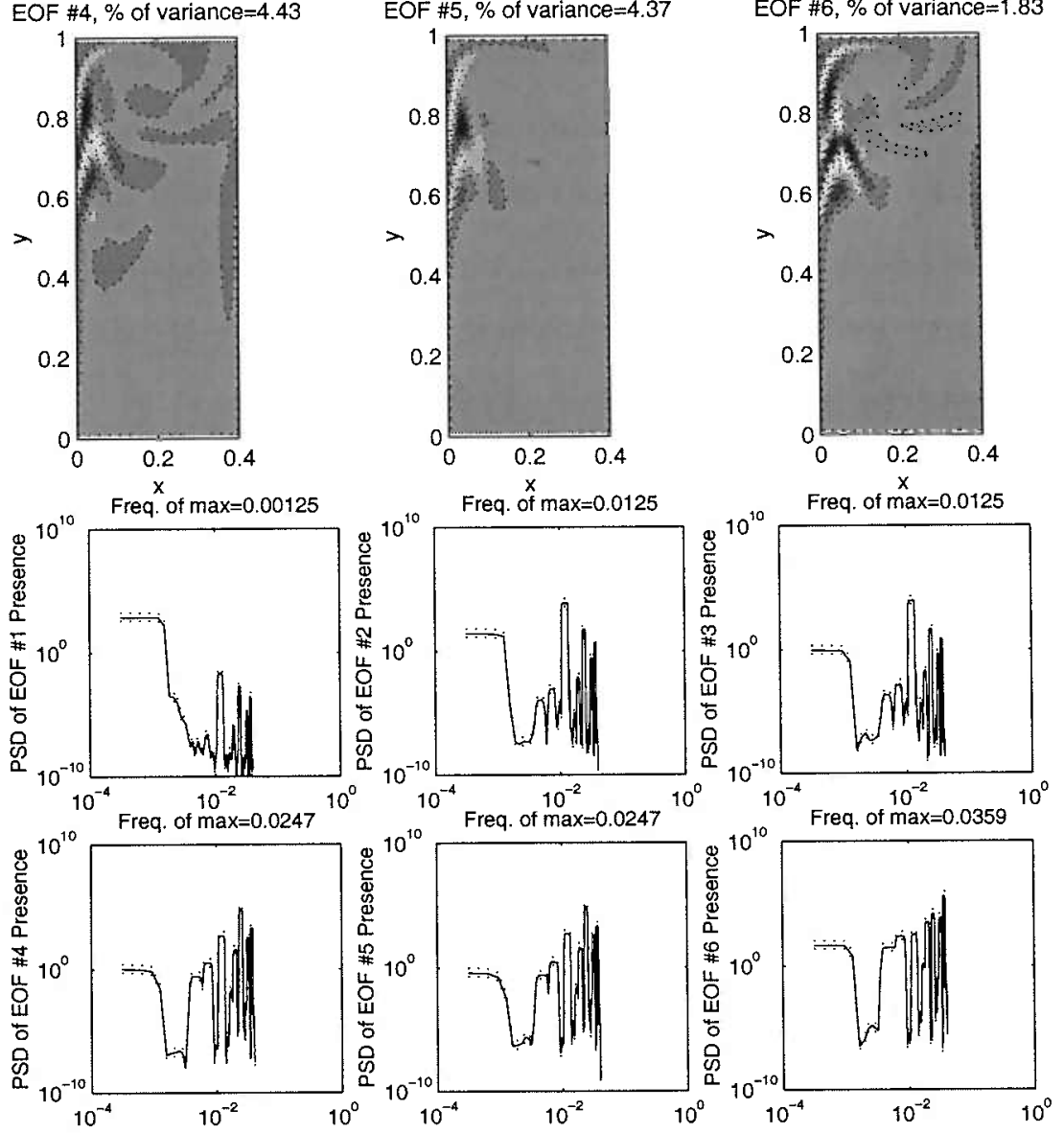

Figure 3-22: EOFs of relative vorticity perturbation from the time-mean as in figure 312 but for the $\operatorname{Re}(\mathrm{bdy})=1, \operatorname{Re}(\mathrm{int})=1$ calculation in a short basin of zonal extent 0.4 . 
strength so that the Sverdrup solution would have the same maximum in the three basins. However, this was not effective, as the boundary current instabilities changed frequency. Thus, while I had designed the 0.4 zonal extent of short basin to tune all of the basin modes below the boundary current instabilities, the resulting boundary current instabilities with wind forcing 2.5 times that in the square basin were at a frequency which was lower than any in either the square or the long basin $(0.0081 \beta L)$. Also, the boundary current was much weaker in this short basin than in either the long basin or the square basin. By increasing the wind forcing to 3.1 times the square basin forcing, I was able to bring the frequencies of the boundary current instabilities closer to those in the calculations in the other basins and above the frequency of the gravest mode $(0.0094 \beta L)$. Unfortunately, the consequence of empirically changing the wind forcing to re-tune the frequency of the boundary current instabilities is that these frequencies can no longer be used to compare to the frequencies in the square basin and the long basin to determine whether the absence of basin modes in the short basin affects the boundary current variability frequencies.

In the short basin, even without the basin modes there are nonlinear interactions similar to those commented upon above regarding figure 3-12, except these interactions are now clearly present in the streamfunction as well as the relative vorticity (figure 3-22). Without basin modes, the structure of the boundary current instabilities and the nonlinear interactions of the eddies in the boundary current can now be observed in the EOFs of the streamfunction as well as in the EOFs of relative vorticity.

It would be a nice confirmation that the counter-rotating gyres were forced by basin modes were they shown to disappear when the basin modes disappear in the short basin calculation. There is no counter-rotating region in the short basin at this Reynolds number. However, there is also no counter-rotating region in the square basin or the long basin at this Reynolds number. This is either due to the fact that the basin modes are weaker at this Reynolds number (they are) or that the increased 
frictional decay rate damps the eddy driven motion. Unfortunately, a higher Reynolds number calculation in the short basin will not yield sensible results because the basin is so short that the recirculation will reach the eastern boundary at a Reynolds number only slightly higher than one.

I also performed a similar comparison between a square basin calculation with $\operatorname{Re}(b d y)=3$ and $\operatorname{Re}($ int $)=3$, and a basin of double the dimension also with $\operatorname{Re}(b d y)=3$ and $\operatorname{Re}(\mathrm{int})=3$. The results in that case were similar in that different basin modes were excited in the larger basin. However, since the length and strength of the boundary current changed (both are approximately doubled in a basin twice as large), the western boundary current instabilities also changed somewhat, making comparison more difficult. However, it is important to note the presence of the basin modes persists even at higher Reynolds number.

In section 3.3 the argument is made that the counter-rotating gyre is likely to be caused by large-scale regions of eddy flux convergence. These regions resembled the nonlinear interaction of one of the basin modes, but do the largest EOFs in a calculation with significant counter-rotating gyres resemble the basin modes in shape and frequency? Figure 3-17 shows the EOFs from the $\operatorname{Re}($ bdy $)=5, \operatorname{Re}($ int $)=5$ calculation which has a pronounced counter-rotating gyre and a pronounced region of eddy flux convergence causing it (see figure 2-6b for the eddy flux convergence and the time-mean streamfunction of this calculation). The gravest basin mode has most of the variance in this calculation, and the $(1,2)$ basin mode is next. The expected pattern of the nonlinear interaction of the gravest basin mode is very similar to the eddy flux convergence pattern. The solution given by Pedlosky (1965b) for the nonlinear steady circulation driven by the $(1,2)$ mode has two counter-rotating gyres below $y=1 / 2$. Since the counter-rotating gyre in the presence of steady wind forcing remains to be addressed, it is unclear at this point which of the two basin modes is responsible for the counter-rotating gyre in this calculation. In either case, there is significant variability in the basin modes, and the expected patterns of eddy 
flux convergence due to the modes are consistent with the existence of a counterrotating region. Pursuing the dynamics of the counter-rotating gyres further than these preliminary remarks is outside the scope of this study.

In summary, the basin modes of oscillation are ubiquitous in these calculations. They play an important role in the dynamics, and they often dominate the variance of the streamfunction perturbations about the mean. It is likely that they force the counter-rotating gyre. They appear to be forced in the region of the turbulent boundary layer, and which modes resonate depends how the frequencies of the instabilities in that region compare to the basin mode frequencies. Because the gravest basin mode has the highest frequency, whether the frequency of this mode is higher or lower than those of the boundary current instabilities determines whether these modes will be present or not.

\subsection{Energetics}

Although most of the discussion in this dissertation deals with the removal of vorticity, the method of removal of kinetic energy from the mean flow as a parametric function of $\operatorname{Re}(\mathrm{int})$ and $\operatorname{Re}(\mathrm{bdy})$ is also helpful in understanding the role of eddies on the mean flow. Importantly, unlike vorticity, the energy can be locally removed, this makes it unclear how enhancing the viscosity in only a thin frictional sublayer will be able to affect the basin-wide energy budget. This section deals with these issues of energetics.

One way to formulate the equation for the kinetic energy of the mean flow is

$$
\begin{gathered}
\frac{\partial \bar{E}}{\partial t}+\nabla \cdot\left[-\bar{\psi} \overline{\frac{D}{D t}} \nabla \bar{\psi}+\delta_{I}^{2} \overline{\mathbf{u}} \bar{E}-\frac{\bar{\psi}^{2}}{2} \hat{\mathbf{x}}+\bar{\psi} \delta_{M}^{3} \nabla \bar{\zeta}-\delta_{S} \nabla \frac{\bar{\psi}^{2}}{2}-\delta_{S} \bar{\psi} \overline{\mathbf{u}}\right] \\
+\nabla \cdot\left[\delta_{I}^{2} \overline{\mathbf{u}} \cdot \overline{\mathbf{u}^{\prime} \mathbf{u}^{\prime}}-\bar{\psi} \delta_{I}^{2} \overline{\mathbf{u}^{\prime} \cdot \nabla \nabla \psi^{\prime}}\right] \\
=\delta_{M}^{3}\left[\nabla^{2} \bar{E}-\left(\frac{\partial^{2} \bar{\psi}}{\partial x^{2}}\right)^{2}-\left(\frac{\partial^{2} \bar{\psi}}{\partial y^{2}}\right)^{2}-2\left(\frac{\partial^{2} \bar{\psi}}{\partial x \partial y}\right)^{2}\right]-2 \delta_{S} \bar{E}+\bar{\psi} \sin (\pi y) \\
+\delta_{I}^{2}\left(\overline{\mathbf{u}^{\prime} \mathbf{u}^{\prime}} \cdot \nabla\right) \cdot \overline{\mathbf{u}} .
\end{gathered}
$$


The terms after the equal sign in this equation are local sinks and sources of energy. The terms proportional to $\delta_{M}^{3}$ are the lateral friction's removal of energy. The term proportional to $\delta_{S}$ is the bottom friction's removal of energy. The term proportional to $\delta_{I}^{2}$ is the production of turbulent kinetic energy. The remaining term is the wind's input of energy to the time-mean flow.

As pointed out in chapter 2, all of the frictional terms are negative definite except for $\delta_{M}^{3} \nabla^{2} E$ which represents a smoothing of sharp energy gradients. However, in this section we will see that the cumulative effect of frictional terms is always to remove wind power upon integration over the basin.

Figure 3-23 shows that the turbulent kinetic energy production rate (as defined in equation 3.10) can be of either sign, and is located primarily at the lower edge of the recirculation gyre. It is important to note that this is not a unique representation of the kinetic energy production rate, as more or less of the flux terms in equation 3.10 could sensibly be added to this term. The basin integral of this term is unique, however, and with increasing $\operatorname{Re}(b d y)$ or $\operatorname{Re}(\mathrm{int})$, the basin-integrated production rate of turbulent kinetic energy increases both in absolute magnitude and relative to the wind power supply to the mean flow. At the same time, the frictional dissipation rate of kinetic energy relative to the wind power decreases with increasing Re(int) and $\operatorname{Re}(\mathrm{bdy})$ (see tables 3.4 and 3.3). Thus, the removal of energy from the mean flow is increasingly due to the effects of eddies as the calculations become more inviscid.

The observations of Webster (1961) measured the local turbulent kinetic energy production (as defined in equation 3.10) to be transferring energy from the meanders to the mean flow in some locations. These observations have often been interpreted as evidence of turbulent eddies strengthening the mean flow of the Gulf Stream. These observations can be understood in in a larger context in figure 3-23. Locally, the production of turbulent kinetic energy can be of either sign, while the basin-wide integral is always in a sense to transfer energy from the mean kinetic energy to the turbulent kinetic energy, as shown in table 3.3 . 

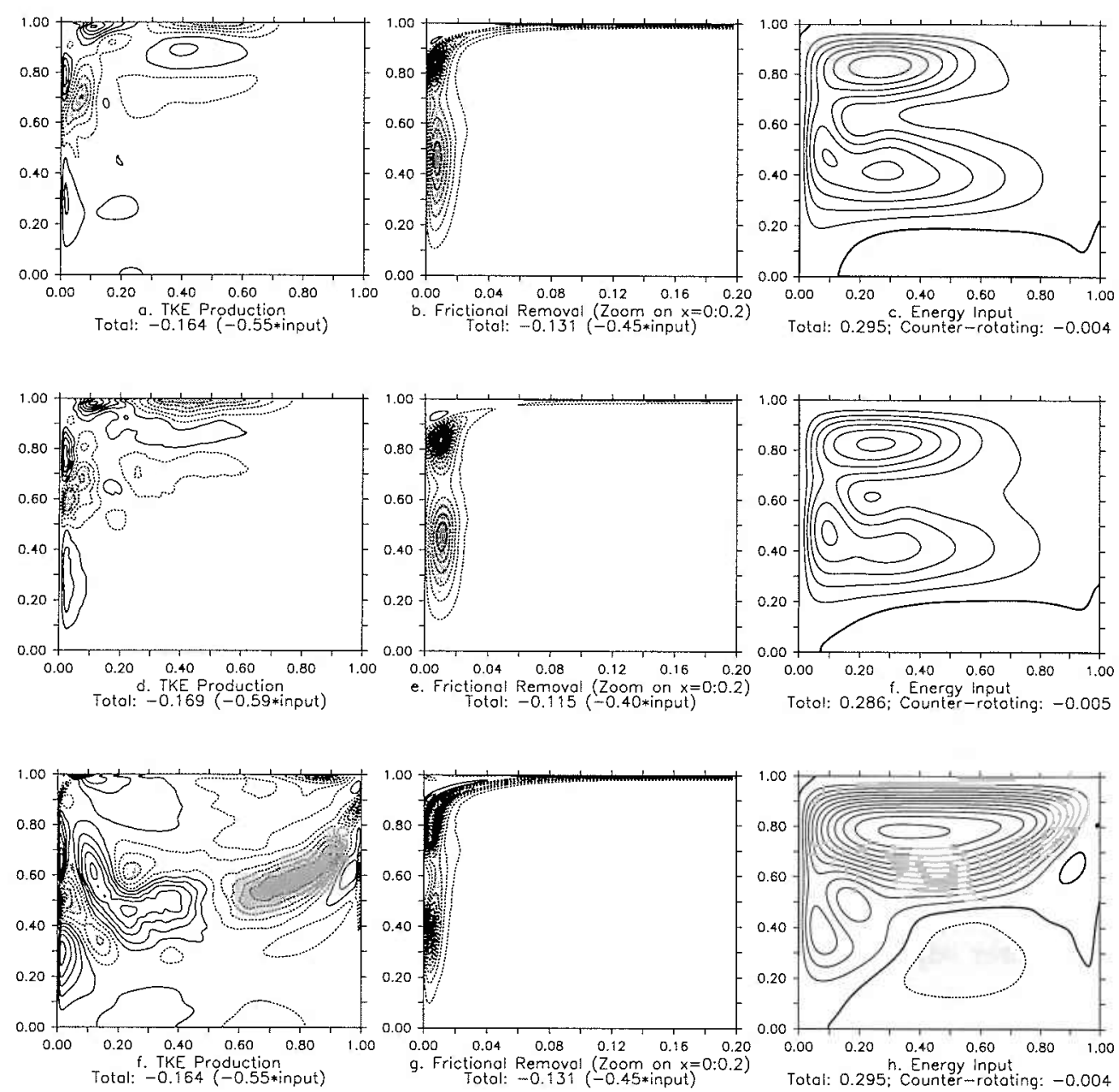

Figure 3-23: Source and sink terms of mean flow kinetic energy as defined in equation 2.14 for the $\operatorname{Re}(\mathrm{bdy})=3, \operatorname{Re}(\mathrm{int})=3$ calculation. (a) shows the turbulent kinetic energy production rate. (b) shows the frictional dissipation rate. (c) shows the wind power. Below each figure the basin-integrated total is given. Below (c) the contribution of the counter-rotating gyre to the wind forcing is also given. Contour intervals are 2 units (centered on 0 ) in (a) and (b) and 0.2 units in (c). (d-e) are the sources and sinks of mean flow kinetic energy as in (a-c), but for the $\operatorname{Re}(\mathrm{bdy})=0.25, \operatorname{Re}($ int $)=8$ calculation. (f-h) are the sources and sinks of mean flow kinetic energy as in (a-c), but for the $\operatorname{Re}(b d y)=5, \operatorname{Re}(i n t)=5$ calculation. The sum of sinks and sources is not exactly zero due averaging errors in the eddy production and time-stepping errors. 
Although it is tempting to interpret the spatial structure of figure 3-23 as indicative of the location of the turbulence's effects on the mean flow, these terms do not describe the full eddy-mean flow interaction because there are other flux terms in the Reynolds-averaged energy equation (equation 3.10) which are equally important. These flux terms vanish upon integration over the basin, so it is sensible to consider the turbulent kinetic energy production as a basin-wide integral. However, it is invalid to ascribe physical meaning to the local value of the production terms without also acknowledging the flux terms. Thus, figure 3-23 is not a unique representation of the terms in the energy equation. This figure was produced only to compare to the results of Webster (1961) (who used similar definitions) and to give some idea of how one representation of the energy equation looks.

As shown elegantly in Plumb (1982), even the physical meaning of the basinintegrated results is tentative, as it depends on what definition of eddy and mean is used. For reasons stated in the introduction, in this dissertation the time-mean is used throughout to define the mean state. Thus, just as the choice of mean importantly determined the meaning of eddy in the vorticity dynamics, the energetics mentioned here are also subject to this definition.

The mean flow energy budget for the entire basin is obtained by integrating the energy equation.

$$
\begin{aligned}
0 & =\iint\left(\delta_{M}^{3}\left[\nabla^{2} \bar{E}-\left(\frac{\partial^{2} \bar{\psi}}{\partial x^{2}}\right)^{2}-\left(\frac{\partial^{2} \bar{\psi}}{\partial y^{2}}\right)^{2}-2\left(\frac{\partial^{2} \bar{\psi}}{\partial x \partial y}\right)^{2}\right]\right) d^{2} A \\
& +\iint\left(-2 \delta_{S} \bar{E}+\delta_{I}^{2}\left(\overline{\mathbf{u}^{\prime} \mathbf{u}^{\prime}} \cdot \nabla\right) \cdot \overline{\mathbf{u}}+\bar{\psi} \sin (\pi y)\right) d^{2} A
\end{aligned}
$$

The energy in the mean flow can be removed locally by lateral or bottom friction or by turbulent kinetic energy production. Tables 3.1, 3.2, 3.3 and 3.4 give numerous details about the basin-integrated energy budgets for the different calculations.

In section 2.2 , it is pointed out that the basin-integrated vorticity budget depended only on the viscosity at the boundary, not in the interior. The energy budget, on the 
other hand, does not obviously single out the value of the viscosity in any particular region, so how is it that boundary-enhanced viscosity also controls the energy of the solution? There are two mechanisms. First, as shown in figure 3-23, the frictional terms are almost exclusively limited to the region of the frictional sublayer. ${ }^{7}$ Thus, increasing the viscosity there will increase the friction energy removal from the mean flow there. A second mechanism is the reduction of wind power. The wind power is proportional to the correlation of wind stress and fluid velocity. Therefore, if the vorticity dynamics are such that the circulation is decreased, then the wind power will also be decreased. Scott and Straub (1998) have proposed a similar scheme previously. Even though the vorticity dynamics are analyzed in terms of fluxes and the energy is analyzed as local sinks, the two are closely linked through the wind power's response to the circulation. Of course, this is only a physical interpretation of the mathematical fact that since the energy equation is formed by multiplying the vorticity equation by $\bar{\psi}$, only the trivial solution of $\bar{\psi}=0$ satisfies one without the other.

Figure 3-23 also compare the energetics of a homoparic pair. The member of each pair with higher Re(int) has a higher rate of removal of energy from the mean flow by turbulent kinetic energy production. For the homoparic pair shown in figure 3-23, the member of the pair with $\operatorname{Re}($ int $)=8$ has only $40 \%$ of its energy input removed by frictional dissipation while the member of the pair with $\operatorname{Re}($ int $)=3$ has $45 \%$ removed by frictional dissipation. Also, the results for the basin-integrated energy budgets for the two homparic pairs compared in section 3.2 are typeset in bold in tables $3.1,3.2$, 3.3 and 3.4 so that they maybe easily compared.

In section 3.2 , the homoparic pair member with higher Re(int) was shown to have more fine scale vorticity features. The eddy field of the homoparic pair member with larger Re(int) is also more energetic and more energetically variable than the pair

\footnotetext{
${ }^{7}$ This form of the frictional dissipation is not unique as frictional fluxes can be added or subtracted. However, all of the frictional terms including the flux convergences are large only in the region of the frictional sublayer.
} 
member with smaller $\operatorname{Re}($ int $)$. For example, the $\operatorname{Re}(\mathrm{bdy})=0.25, \operatorname{Re}(\mathrm{int})=8$ calculation has a total kinetic energy of 127 while the $\operatorname{Re}(b d y)=3, \operatorname{Re}($ int $)=3$ calculation has a total kinetic energy of 102 (see table 3.1). This is true even though the kinetic energy of the mean flows of these calculations are 30.9 and 37.7 , respectively (see table 3.2). The variability of the total kinetic energy is also larger in the pair member with larger Re(int) (see table 3.1). By examining tables 3.1 and 3.2, it becomes clear that the same is true of the $\operatorname{Re}(\mathrm{bdy})=0.5, \operatorname{Re}(\mathrm{int})=3$ and $\operatorname{Re}(\mathrm{bdy})=0.25, \operatorname{Re}($ int $)=5$ homoparic pair. So, examining the energetics of the homoparic pairs reveals that the pair member with larger Re(int) has more energy which is also more variable and its turbulent kinetic energy production is responsible for removing a greater portion of the wind power applied to it.

The wind power term can be of either sign, as the work done differs depending on the solution's alignment with the wind stress. In particular, in the region of the counter-rotating gyres the wind power removes energy from the basin. Figure 3$23 \mathrm{c}$ shows the energetics of an inertially-dominated calculation. In section 3.3 it is proposed that perhaps the existence of the counter-rotating gyres can be explained by their reduction of the wind power input. Interestingly, despite a large and pronounced counter-rotating gyre, the energy input to the circulation is decreased by slightly less than $10 \%$ by this gyre when compared to the power that would be applied to a solution with the same streamfunction everywhere except in the counter-rotating region where $\psi$ is set to zero. In all of the inertially-dominated equilibria, the bulk of the removal of energy is performed by the turbulent kinetic energy production. However, if one were to fill the entire basin with a single gyre rotating in the same direction as the wind, this would likely represent a more significant increase depending on the strength of the basin-filling circulation.

The results in the tables and graphs in this section are particularly subject to numerical errors including errors in averaging of the temporally chaotic calculations. See section C.5 for a discussion of the errors. 


\begin{tabular}{|l|l|l|l|l|l|l|}
\hline $\operatorname{Re}($ bdy) $=$ & 0.25 & 0.5 & 1 & 3 & 5 & 7 \\
\hline $\operatorname{Re}($ int $)=0.5$ & & 5.5 & 6.3 & & & \\
& & $(5.5-5.5)$ & $(6.3-6.3)$ & & & \\
\hline $\operatorname{Re}($ int $)=1$ & 6.5 & 8.2 & 11.2 & & & \\
& $(6.5-6.6)$ & $(8.1-8.2)$ & $(11.1-11.2)$ & & & \\
\hline $\operatorname{Re}($ int $)=3$ & 20.5 & $\mathbf{3 1 . 0}$ & 63.6 & 102 & & \\
& $(17.5-23.8)$ & $(23.6-37.8)$ & $(41.8-84.8)$ & $(87.2-110.7)$ & & \\
\hline $\operatorname{Re}($ int $)=5$ & $\mathbf{4 9 . 6}$ & 91.8 & 253 & 334 & 342 & \\
& $(32.0-65.8)$ & $(60.1-118)$ & $(249-256)$ & $(329-342)$ & $(329-357)$ & \\
\hline $\operatorname{Re}($ int $)=7$ & 89.0 & 280 & 323 & 400 & & 439 \\
& $(67.7-101)$ & $(260-293)$ & $(308-349)$ & $(396-407)$ & & $(429-449)$ \\
\hline $\operatorname{Re}($ int $)=8$ & $\mathbf{1 2 7}$ & 298 & & & & \\
& $(82.6-158)$ & $(289-312)$ & & & & \\
\hline $\operatorname{Re}($ int $)=9$ & 276 & & & & & \\
& $(249-296)$ & & & & & \\
\hline
\end{tabular}

Table 3.1: Time-mean of total kinetic energy value and range of variability (in parentheses) after spin-up as a function of $\operatorname{Re}(b d y)$ and Re(int). Compare with figure 3-11.

\begin{tabular}{|l|l|l|l|l|l|l|}
\hline $\operatorname{Re}($ bdy $)=$ & 0.25 & 0.5 & 1 & 3 & 5 & 7 \\
\hline $\operatorname{Re}($ int $)=0.5$ & & 5.5 & 6.3 & & & \\
\hline $\operatorname{Re}($ int $)=1$ & 6.1 & 6.7 & 8.3 & & & \\
\hline $\operatorname{Re}($ int $)=3$ & 8.1 & $\mathbf{1 3 . 5}$ & 19.7 & $\mathbf{3 7 . 7}$ & & \\
\hline $\operatorname{Re}($ int $)=5$ & $\mathbf{1 4 . 0}$ & 30.5 & 91.1 & 109.8 & 106.4 & \\
\hline $\operatorname{Re}($ int $)=7$ & 24.2 & 87.4 & 96.2 & 114.1 & & 109.9 \\
\hline $\operatorname{Re}($ int $)=8$ & $\mathbf{3 0 . 9}$ & 86.7 & & & & \\
\hline $\operatorname{Re}($ int $)=9$ & 80.8 & & & & & \\
\hline
\end{tabular}

Table 3.2: Kinetic energy of time-mean flow as a function of $\operatorname{Re}(\mathrm{bdy})$ and $\operatorname{Re}(\mathrm{int})$.

\begin{tabular}{|l|l|l|l|l|l|l|}
\hline $\operatorname{Re}($ bdy $)=$ & 0.25 & 0.5 & 1 & 3 & 5 & 7 \\
\hline $\operatorname{Re}($ int $)=0.5$ & & $0 \%$ & & & & \\
\hline $\operatorname{Re}($ int $)=1$ & $7 \%$ & $16 \%$ & $16 \%$ & & & \\
\hline $\operatorname{Re}($ int $)=3$ & $41 \%$ & $\mathbf{4 3 \%}$ & $49 \%$ & $\mathbf{5 5 \%}$ & & \\
\hline $\operatorname{Re}($ int $)=5$ & $\mathbf{4 8 \%}$ & $59 \%$ & $54 \%$ & $61 \%$ & $61 \%$ & \\
\hline $\operatorname{Re}($ int $)=7$ & $55 \%$ & $60 \%$ & $62 \%$ & $63 \%$ & & $72 \%$ \\
\hline $\operatorname{Re}($ int $)=8$ & $\mathbf{5 9 \%}$ & $59 \%$ & & & & \\
\hline $\operatorname{Re}($ int $)=9$ & $60 \%$ & & & & & \\
\hline
\end{tabular}

Table 3.3: Basin-integrated percentage of power input to mean flow removed by turbulent kinetic energy production as a function of $\operatorname{Re}(b d y)$ and $\operatorname{Re}(\mathrm{int})$. This is an independent calculation from table 3.4 , so the extent to which the two do not add up to $100 \%$ indicates the typical error. 


\begin{tabular}{|l|l|l|l|l|l|l|}
\hline $\operatorname{Re}($ bdy) $=$ & 0.25 & 0.5 & 1 & 3 & 5 & 7 \\
\hline $\operatorname{Re}($ int) $=0.5$ & & $100 \%$ & & & & \\
\hline $\operatorname{Re}($ int) $=1$ & $93 \%$ & $84 \%$ & $84 \%$ & & & \\
\hline $\operatorname{Re}($ int $)=3$ & $60 \%$ & $57 \%$ & $51 \%$ & $\mathbf{4 5 \%}$ & & \\
\hline $\operatorname{Re}($ int $)=5$ & $\mathbf{5 0 \%}$ & $44 \%$ & $44 \%$ & $41 \%$ & $39 \%$ & \\
\hline $\operatorname{Re}($ int $)=7$ & $43 \%$ & $39 \%$ & $39 \%$ & $34 \%$ & & $33 \%$ \\
\hline $\operatorname{Re}($ int) $=8$ & $\mathbf{4 0 \%}$ & $39 \%$ & & & & \\
\hline $\operatorname{Re}($ int $)=9$ & $40 \%$ & & & & & \\
\hline
\end{tabular}

Table 3.4: Basin-integrated percentage of power input to mean flow removed by lateral friction as a function of $\operatorname{Re}(b d y)$ and $\operatorname{Re}(i n t)$.

In summary, the energy budget of the mean and eddy flow reveals that the eddies are increasingly responsible for the removal of wind power as the solutions become more inviscid. This is true even for the homoparic pairs, where the member with larger Re(int) has more energy, more variability in energy, and more removal of wind power by turbulent kinetic energy production. The spatial distribution of energy terms is complicated, non-trivial and potentially misleading because of the lack of unique definitions of energy transfer. This complexity is the reason that an emphasis is placed on vorticity dynamics in this dissertation as opposed to energetics.

\subsection{Bottom Friction}

Some calculations with bottom friction were also performed so that the results in this dissertation can be placed in context with other calculations using bottom friction. The effects of bottom friction seen here are making the flow laminar, fluxing vorticity across mean streamlines (especially in the recirculation gyre), reducing the amount of large-scale variability, and removing a small amount of vorticity through the slip boundaries. It is the relative sizes of the boundary layer scales which determine how the addition of bottom friction will affect the solution.

If either the Stommel width or the Munk width is larger than the Charney width, the solution is essentially linear, closely resembling the Stommel (1948) and Munk 
(1950) solutions. Actually, Munk and Carrier (1950) calculated solutions to this problem on a mechanical computer!

If the Stommel width is larger than the Munk width, at least in these simulations, it always tended to make the flow laminar, regardless of the Charney length. This is likely due to the fact that the shear caused by the lateral friction is crucial to eddy formation in this model. This agrees with Veronis (1966b), who studied the inertial Stommel model with no lateral friction and found steady solutions. However, unlike in Veronis' calculations, there is always a lateral friction sublayer in these calculations. The no-slip boundary condition requires that the sublayer change the shear very near the boundary significantly. Thus, unlike in Veronis' calculation the solution may satisfy both the Fjørtoft (1950) instability criterion. The Charney and Stern (1962) instability criterion appears to always be satisfied in these calculations. Thus, it is possible that if it were numerically feasible to make a calculation with a greater separation between the frictional scales and the Charney scale then the solution might not be laminar. It appears that here, however, for all of the cases where $\delta_{I}>\delta_{S}>\delta_{M}$, the damping rate of the bottom friction is just too rapid for the instabilities to arise. In the laminar or nearly laminar solutions, the eddies play little role, and the dynamics are essentially those of Veronis (1966a), except with a lateral friction sublayer where the frictional boundary conditions are achieved.

If the Stommel width is slightly smaller than the Munk width, the bottom friction's primary effect is still to decrease the amount of variability. To demonstrate the subtle interplay of the bottom friction and the lateral friction in eddy production consider figure 3-24. Figure 3-24 shows the mean streamfunctions and eddy flux convergences for three calculations with bottom friction as well as lateral friction. Figure 3-24a has some important eddy activity and is western-intensified, while figure 3-24b has very little eddy activity and has a large recirculation gyre. Interestingly figure 3-24a has the same bottom friction as figure 3-24b, and has a larger lateral viscosity near the boundary. In an unintuitive result, raising the viscosity produced a greater number, 
not fewer, eddies in this calculation.

Note that the laminar solutions may not be very western-intensified despite a relatively large Stommel width. In figure 3-24b there is very little eddy activity when compared to figure $3-24 \mathrm{c}$. It is also true that figure $3-24 \mathrm{~b}$ is roughly as westernintensified as figure $3-24 \mathrm{c}$. However, figure $3-24 \mathrm{~b}$ is more viscous than figure $3-24 \mathrm{c}$. Thus, the Stommel width does not have a huge effect on western-intensification when it is smaller than the Munk width.

If the Stommel width is much smaller than the Munk width, and both are smaller than the Charney length, the bottom friction plays two roles. in figure $3-25 \mathrm{c}$ and in figure $3-24 \mathrm{c}$, the bottom friction is very small, and the eddies are quite active. The flux across mean streamlines is shown in figure $3-25 \mathrm{c}$.

The bottom friction's first role when $\delta_{S} \ll \delta_{M}$ is that it does remove a small amount of vorticity from the basin (as anticipated by equation 2.16). Although the no-slip boundary conditions guarantee that the bottom friction removes no vorticity through the eastern and western boundaries, it can and does remove a small amount through the northern and southern boundaries. This is indicated by the non-zero value of the bottom friction flux at $\bar{\psi}=0$ in figures $3-25$.

In figure 3-25, the cross-streamline flux of vorticity carried by the bottom friction first increases then decreases with $\bar{\psi}$. This result submits to simple interpretation. Because of the no-slip boundary conditions on the eastern and western boundaries, the bottom friction cannot remove vorticity there. So, like the eddy flux of vorticity, the bottom friction transfers its vorticity transport to the lateral friction in a sublayer near the no-slip boundary to be removed. This transfer results in a divergence of the bottom friction vorticity flux as $\psi$ approaches zero, which is what causes the change from increasing to decreasing flux magnitude with decreasing $\bar{\psi}$ in figure $3-25$. Even a linear solution of the homogeneous model with both bottom and lateral friction exhibits this behavior.

The second, less obvious role of the bottom friction when $\delta_{S} \ll \delta_{M}$ is that it 

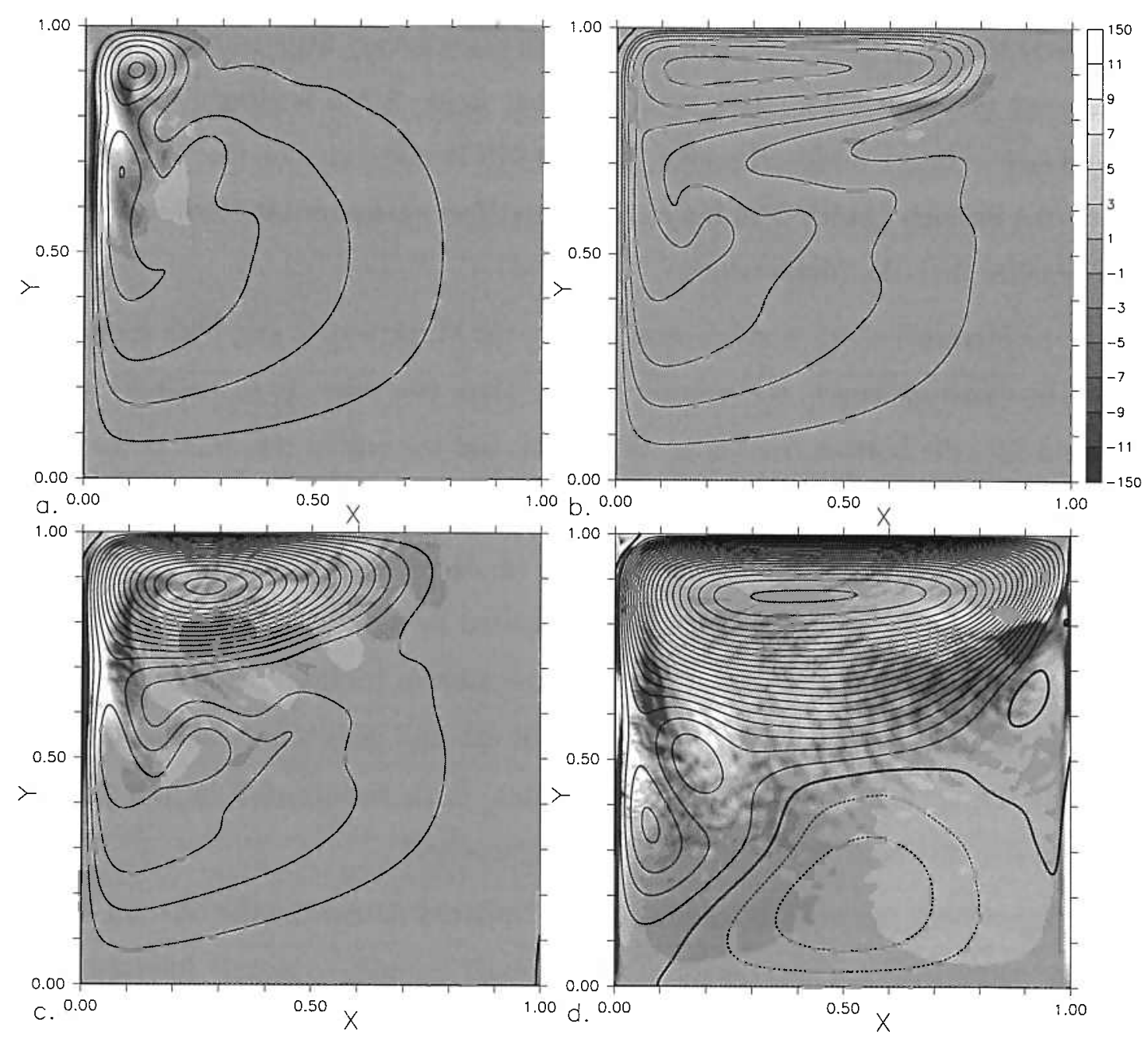

Figure 3-24: (a) The time-mean streamfunction contours are superimposed on the eddy flux convergence shaded for the $\operatorname{Re}(\mathrm{bdy})=0.25$, $\operatorname{Re}($ int $)=5$, no-slip calculation with bottom friction $\left(\delta_{M}(\mathrm{bdy})=0.0317, \delta_{M}(\mathrm{int})=0.0117, \delta_{S}=0.003\right)$. Lighter shading denotes convergences (which remove vorticity of the sign of the wind input); darker shading denotes divergences. (b) is similar to (a) but for $\operatorname{Re}(\mathrm{bdy})=5$, $\operatorname{Re}($ int $)=5\left(\delta_{M}\right.$ (bdy) $=0.0117, \delta_{M}($ int $\left.)=0.0117, \delta_{S}=0.003\right) . \quad(\mathrm{c})$ is $\left(\delta_{M}(\mathrm{bdy})=0.0117\right.$, $\left.\delta_{M}(\mathrm{int})=0.0117, \delta_{S}=0.001\right)$. For comparison $(\mathrm{d})$ shows the $\operatorname{Re}(\mathrm{bdy})=5, \operatorname{Re}(\mathrm{int})=5$ calculation without bottom friction $\left(\delta_{M}(\mathrm{bdy})=0.0117, \delta_{M}(\mathrm{int})=0.0117, \delta_{S}=0\right) . \delta_{I}=0.02$ for all the calculations. 

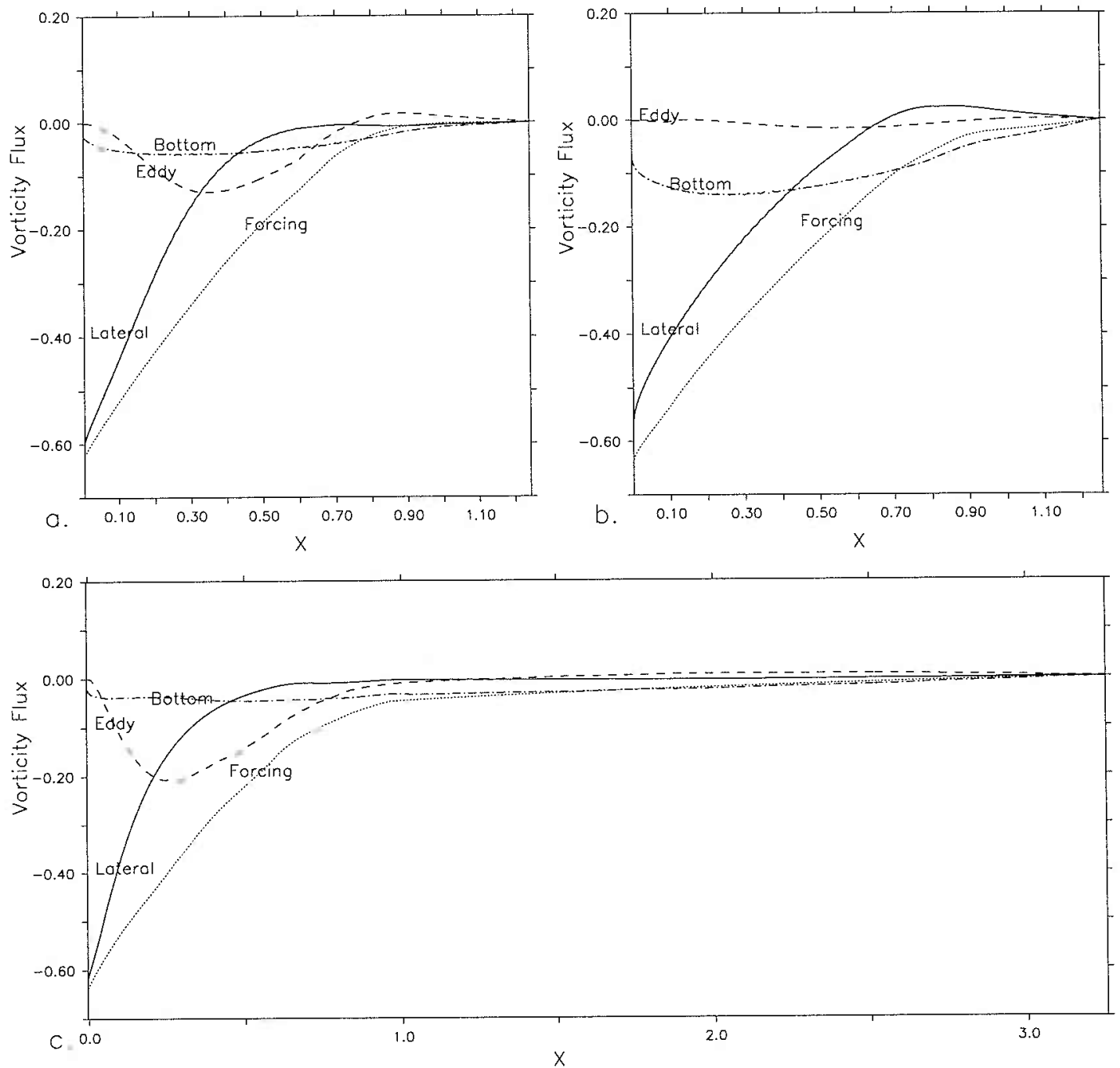

Figure 3-25: These plots show the vorticity flux across mean streamlines for three no-slip calculations with bottom friction. Shown are the eddy flux outward which is $\left(\oint_{\psi_{c}} \overline{\mathbf{u}^{\prime} \zeta^{\prime}} \cdot \hat{\mathbf{n}} d s\right)$, the lateral friction flux outward which is $\left(-\oint_{\psi_{c}} \delta_{M}^{3} \nabla \bar{\zeta} \cdot \hat{\mathbf{n}} d s\right)$, the bottom friction flux outward which is $\left(\oint_{\psi_{c}} \delta_{S} \nabla \bar{\psi} \cdot \hat{\mathbf{n}} d s\right)$, and the wind forcing within that streamline which is $\left(-\iint_{\psi \leq \psi_{c}} \sin (\pi y) d^{2} A\right)$. The runs shown in (a-c) as those in figure 3-24a-c. 
is the primary mode of transport across mean streamlines in the recirculation gyre. This is true even in the presence of a strong eddy flux. In fact, in figures 3-25a and $3-25 \mathrm{~b}$, the bottom friction flux is greater than the forcing, which requires an eddy flux or a lateral friction flux to add vorticity to strengthen the recirculation to compensate. This peculiar overcompensation appears to vanish for smaller Stommel widths as in figure $3-25 \mathrm{c}$, but it is plain that it is easier to remove vorticity from the recirculation gyre by bottom friction than by eddy fluxes. Comparing figure 3-24c to figure $3-24 \mathrm{~b}$, it is clear that not only is there less eddy activity in the case with larger bottom friction, but also the recirculation gyre is weaker by a factor of nearly three. The larger bottom friction damps the recirculation gyre by making the transfer of vorticity across streamlines more efficient.

Note the role of the lateral friction sublayer when $\delta_{S} \ll \delta_{M}$. It is accepting and removing through the western boundary almost as much vorticity flux from the bottom friction flux and eddy flux in figure $3-24 \mathrm{c}$ as it is in figure 3-24d (0.61 versus $0.70)^{8}$ However, the removal of this vorticity goes much more smoothly in figure $3-$ $24 \mathrm{c}$ than it does in figure $3-24 \mathrm{~d}$. As the lateral viscosity is the same in these two calculations, this must have to do with the gradient of $\bar{\zeta}$ in the frictional sublayer.

Figure 3-26 shows $\bar{\zeta}$ as a function of $x$ within the frictional sublayer at a number of points along the boundary. In the calculation with bottom friction, $\bar{\zeta}$ is always decreasing or constant as one proceeds away from the boundary, so the frictional flux is always out of the basin. In the calculation without bottom friction, the gradient of $\bar{\zeta}$ can be of either sign, depending on where in the sublayer it is evaluated.

The changes in the sign of the gradient of $\bar{\zeta}$ are related to the removal crisis and reversal of sign of the eddy flux divergence in the sublayer which was pointed out in section 2.3.2 and to the small vortex trapped in the northwest corner also pointed out in section 2.3.2. Apparently, these effects are significantly weaker when bottom friction is present. Perhaps the instabilities which form the eddies were the cause of

\footnotetext{
${ }^{8}$ The total vorticity removed from the basin by all frictional fluxes is 0.64 , these are estimates of the flux through only the western boundary by lateral friction.
} 

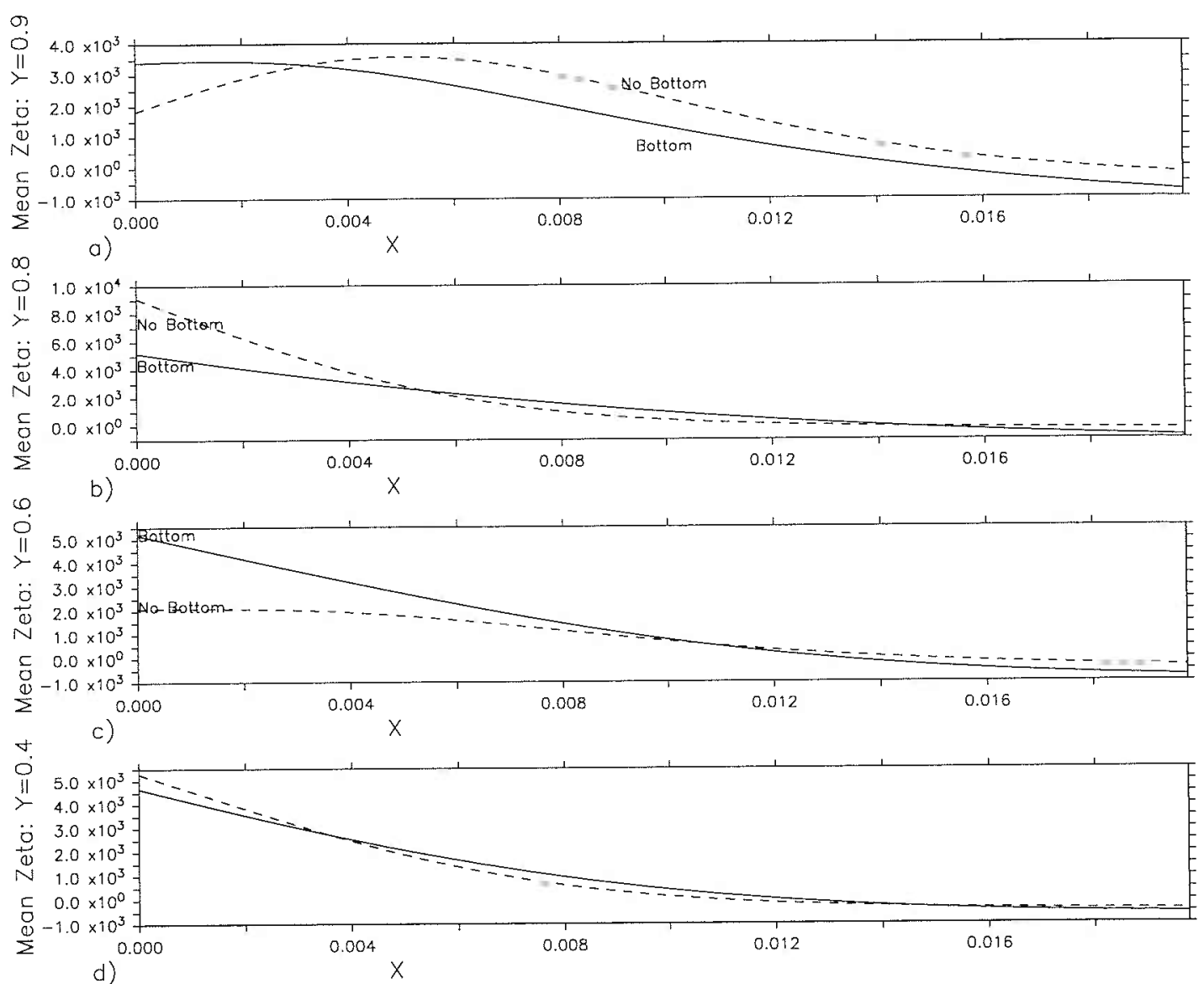

Figure 3-26: These plots show $\bar{\zeta}$ for the calculations shown in figures $3-24 \mathrm{c}-\mathrm{d}$ as a function of $x$ within the western boundary's frictional sublayer at different values of $y$ : (a) $y=0.9$, (b) $y=0.8$, (c) $y=0.6$, and (d) $y=0.4$. Solid lines denote the calculation with bottom friction (from figure 3-24c) and dashed lines denote the calculation without bottom friction (from figure 3-24d). The lateral viscosity is the same for the two calculations. 
the removal crisis as the vorticity gradient increased with Reynolds number. In this case, the bottom friction may have stabilized or reduced these modes.

Another possibility is that it is an overgrown recirculation gyre which begins the removal crisis. The efficient transport of vorticity out of the recirculation gyre by bottom friction appears to be an effective counter to the growth of a strong recirculation gyre in figure 3-24.

It is very important to the discussion in section 4.4.4 to note that it is possible to change or postpone the removal crisis by changing the other ingredients in the crisis other than the lateral friction.

The third effect of bottom friction when $\delta_{S} \ll \delta_{M}$ is that since the bottom friction is not scale selective (unlike the lateral friction) all of the modes of instability, including the global basin modes, are damped equally rapidly by bottom friction. This greatly weakened or removed the counter-rotating gyres from the calculations shown in figure 3-24, because they are forced by these large-scale modes. In the case of lateral friction only, these modes are relatively inviscid due to their large scale. So, a calculation with both lateral friction and bottom friction has weaker large-scale time-dependent features as compared to a calculation with lateral friction only.

All of the effects of bottom friction may be summarized by contrasting figure 3$24 \mathrm{~d}$ with figure $3-24 \mathrm{c}$. These two calculations have the same lateral friction viscosity and inertial boundary layer width. Figure 3-24d has more eddy activity, including large-scale modes which cause a counter-rotating gyre. Its recirculation gyre is much stronger (indicating a lack of the efficient bottom friction flux across mean streamlines there). Finally, the circulation of figure 3-24d is significantly stronger, in small part due to the lack of vorticity removal by bottom friction through the northern and southern boundaries. 


\subsection{Slip Boundary Conditions}

In the other sections of this chapter, and in all of chapter 2, the calculations used no-slip boundary conditions $\left(\frac{\partial \psi}{\partial n}=0\right)$ on the eastern and western boundaries. In this section, and also in some of Chapter 4 , slip boundary conditions $(\zeta=0)$ on all sides of the basin will be used for comparison. Changing the boundary conditions typically brings about a large difference in the solutions' character. Some of the differences found here will be explored in this section, while others that involve the interaction between multiple gyres will be explored in the next chapter.

Barotropic calculations with slip boundary conditions transition to inertiallydominant solutions at a much lower Reynolds number than do no-slip calculations. This is shown clearly in Sheremet et al. (1997). This result has also been confirmed using the steady-state models described in Appendix B. When eddies are permitted, the slip calculations remain more prone to inertial domination; Böning (1986) showed an example of a time-dependent inertially-dominated solution with $\operatorname{Re}($ int $)=\operatorname{Re}(b d y)=1.5$.

However, enhancing the frictional sublayer still has an effect, though a smaller value of $\operatorname{Re}(\mathrm{bdy})$ must be used for a given value of $\operatorname{Re}(\mathrm{int})$ to prevent inertial dominance. Figure 3-27(a-b) show the time-mean of the streamfunction of a westernintensified and an inertially-dominated solution with the same value or $\operatorname{Re}(\mathrm{int})$ and slip boundary conditions. The streamfunctions are superimposed on the eddy flux convergences.

Compare figure 3-27a with figure 3-27c. The former is a slip boundary condition calculation with $\operatorname{Re}(\mathrm{bdy})=0.1$ and $\operatorname{Re}(\mathrm{int})=2.5$. The latter is a no-slip boundary condition calculation with $\operatorname{Re}(\mathrm{bdy})=0.5$ and $\operatorname{Re}(\mathrm{int})=5$. The mean fields are surprisingly similar given the different boundary conditions. Also, although the mean dynamics are quite different, the pattern of the eddy flux convergence field is very similar. The magnitudes of the eddy fluxes are quite different, but the similarity of the patterns, including even the interactions with the recirculation gyre, is stunning. Apparently, 

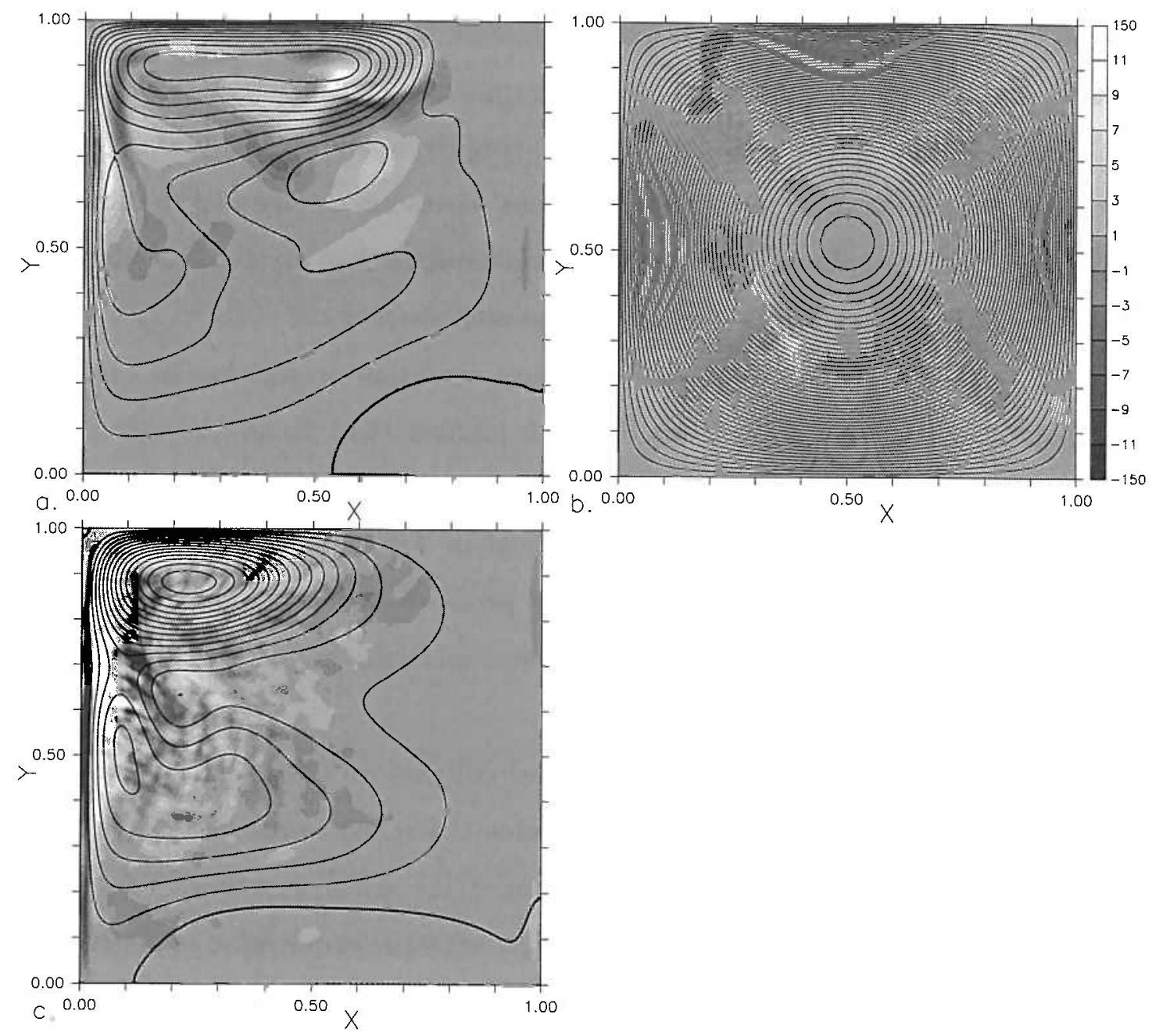

Figure 3-27: (a) The time-mean streamfunction contours are superimposed on the eddy flux convergence shaded for the $\operatorname{Re}(\mathrm{bdy})=0.1$, $\operatorname{Re}(\mathrm{int})=2.5$, slip boundary condition calculation. Lighter shading denotes convergences (which remove vorticity of the sign of the wind input); darker shading denotes divergences. (b) is similar to (a) but for $\operatorname{Re}(\mathrm{bdy})=2.5, \operatorname{Re}(\mathrm{int})=2.5$. (c) shows a no-slip calculation $(\operatorname{Re}(\mathrm{bdy})=0.5$, $\operatorname{Re}($ int $)=5$ ) which is qualitatively similar to the slip calculation shown in (a). Contour interval is 0.2 for (a) and (c) and 20 for (b). 
there is enough which is similar between these solutions that the structure of the eddy field can take a similar form. Even the counter-rotating gyres are present in the slip boundary condition calculations, despite the fact that the eddy activity is not nearly as strong in figure 3-27a as in the no-slip calculations such as in figure 3-27c.

Figure 3-28 shows that, at least in the boundary-enhanced viscosity case, the cross-streamline flux of vorticity is still carried by the eddy flux for streamlines where $\psi$ is greater than approximately 0.6. Thus, the eddies are not doing as much of the work in the slip calculation as in the no-slip calculations, but they still remove almost all of the vorticity input to the recirculation gyre. Because the slip boundary conditions do not easily generate eddy activity, it is in fact possible to reach a steadystate inertially-dominated solution in some cases. This accentuates the critical role of the eddies in fluxing vorticity to the boundary. Figure $3-27 \mathrm{~b}$, which is steady, has transport more that 800 times the linear Munk solution's transport. Without the eddies, the circulation must be extremely strong for the direct action of lateral viscosity to reach the innermost streamlines at high Reynolds number.

The reduced eddy activity may be attributable to the reduced vorticity gradients. In figure 3-29, the absolute vorticity of the three calculations presented in figure 3-27 are contoured. Neither of the slip calculations has comparable vorticity gradients to those found in the western boundary layer of the no-slip calculation. Given that the stability criterion for shear instability depends directly on the absolute vorticity gradient, it is no surprise that slip calculations have weaker eddy activity. Although, all of these calculations, including the steady one, satisfy the Charney and Stern (1962) and Fjørtoft (1950) necessary (but not sufficient!) instability criteria. More information on these necessary criteria and frictional instabilities can be found in texts such as Drazin and Reid (1981).

Viscous instabilities, such as the Tollmien-Schlichting waves and those described in Ierley and Young (1991b) and Ierley (1993) are also sensitive to the vorticity gradients near the boundary. Ierley and Young (1991b) found that the onset of these instabilities 

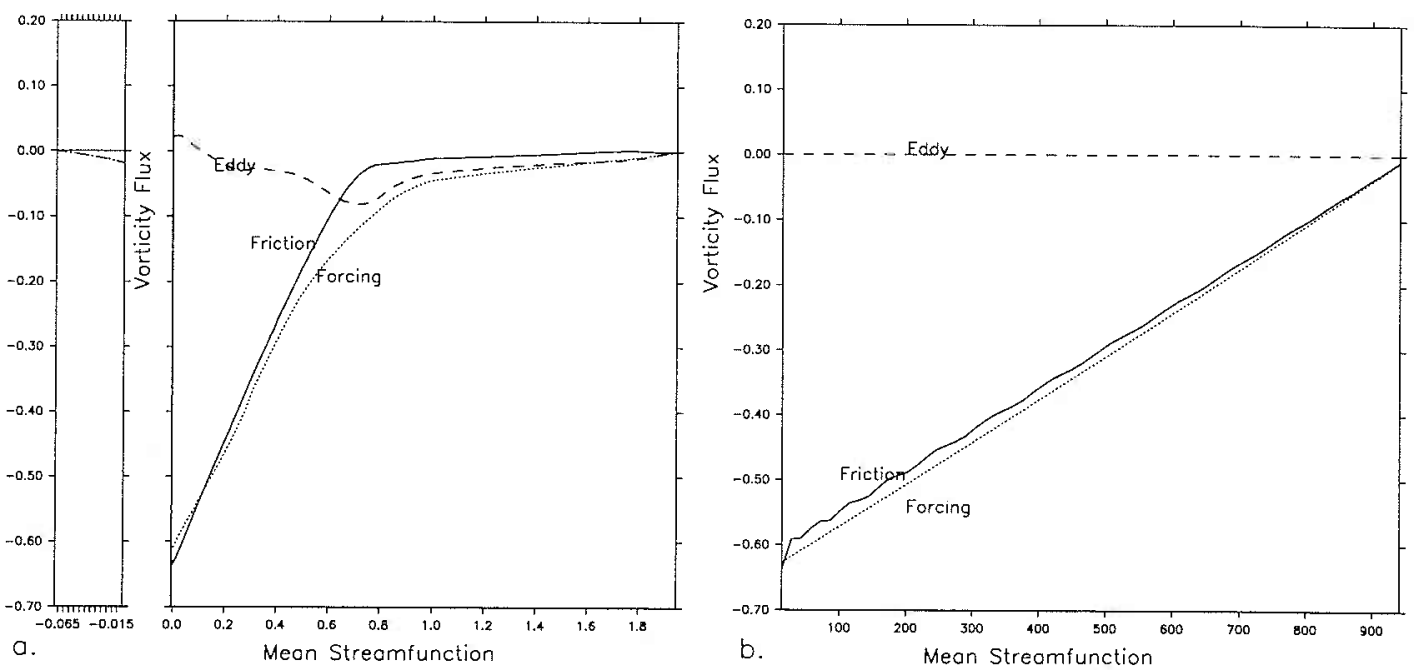

Figure 3-28: These plots show the vorticity flux across mean streamlines for two calculations with slip boundary conditions. Shown are the eddy flux outward which is $\left(\oint_{\psi_{c}} \overline{\mathbf{u}^{\prime} \zeta^{\prime}} \cdot \hat{\mathbf{n}} d s\right)$, the friction flux outward which is $\left(-\oint_{\psi_{c}} \delta_{M}^{3} \nabla \bar{\zeta} \cdot \hat{\mathbf{n}} d s\right)$, and the wind forcing within that streamline which is $\left(-\iint_{\psi \leq \psi_{c}} \sin (\pi y) d^{2} A\right)$. (a) shows the result for the $\operatorname{Re}(\mathrm{bdy})=0.1, \operatorname{Re}(\mathrm{int})=2.5$ calculation. (b) shows the result for the $\operatorname{Re}(\mathrm{bdy})=2.5$, $\operatorname{Re}($ int $)=2.5$ calculation. See figure 3-27 for location of mean streamlines for these calculations. The sum of the frictional fluxes at the boundary is the frictional removal from the basin. The sum of the eddy fluxes at the boundary is zero. See section 2.2.1 for details.

occurred at a much smaller Reynolds number with no-slip boundary conditions than with slip boundary conditions. The results here are consistent with this result.

The reduced generation of eddy activity near the boundary in the slip calculations is the most significant difference between the slip and no-slip calculations from the perspective of this dissertation. In Chapter 4, the lack of boundary-produced eddies in the slip calculation leads to an entirely different behavior for the slip and no-slip calculations. It seems likely that a realistic oceanic boundary would produce many eddies, so in this respect it seems that the no-slip boundary condition is better for reproducing oceanic conditions.

The enhanced frictional sublayer is once again able to prevent inertial domination in the case of slip boundary conditions, although the eddy fluxes do not carry the 

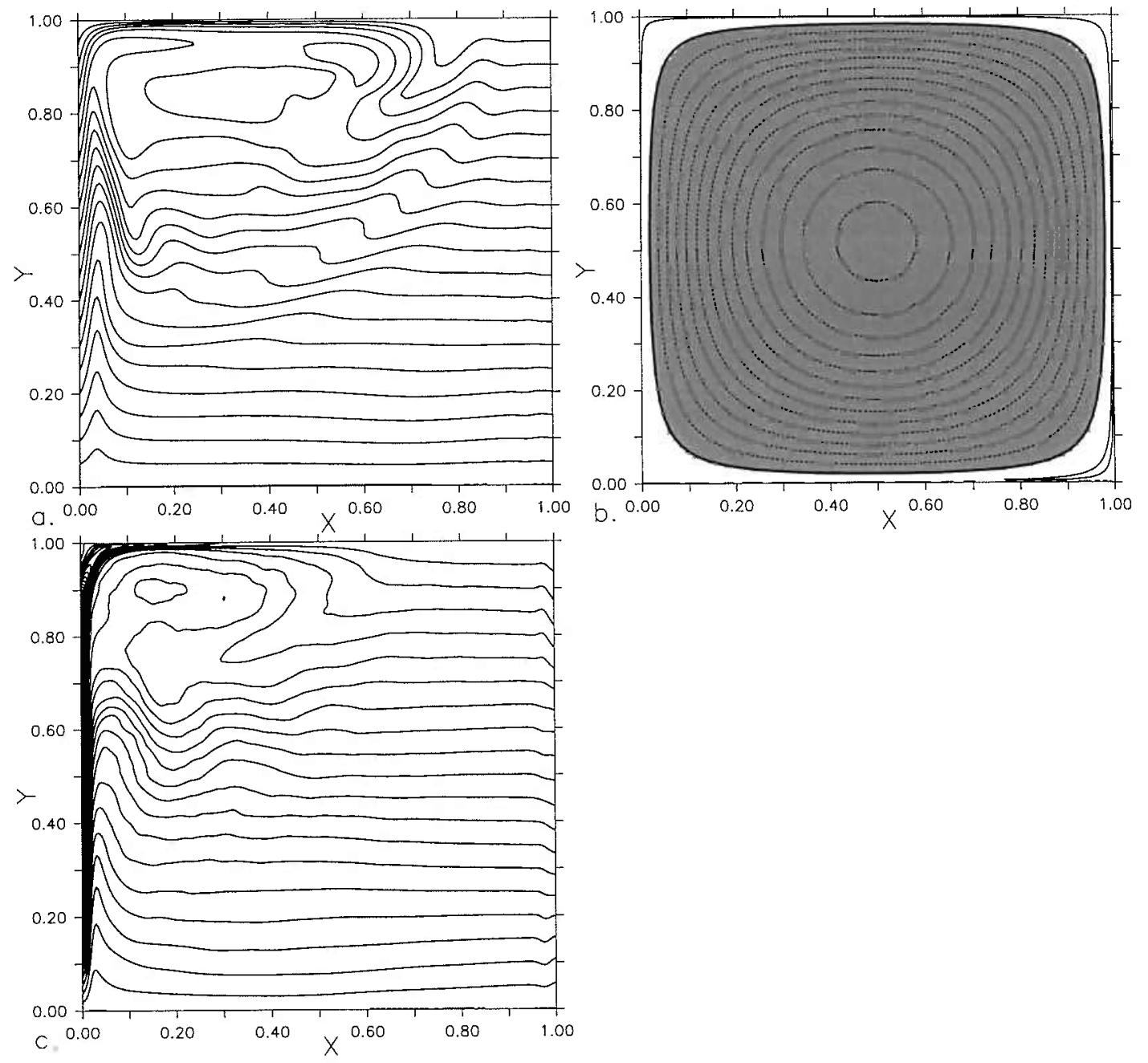

Figure 3-29: These plots show the absolute vorticity $\left(\delta_{I}^{2} \zeta+y\right)$ for the calculations in figure 3-27. The figure letters correspond to the results from the same calculations. The contour interval is 0.05 for (a) and (c) and 0.5 for (b). Regions of negative vorticity are shaded. 
vorticity as close to the boundary as in the no-slip calculations, and the eddies are significantly weaker. This is likely to be due to the fact that the absolute vorticity gradients are not as strong in the boundary current with slip boundary conditions. Thus, there are far too few eddies formed near the boundary with slip boundary conditions. Quite surprisingly, considering the qualitative differences between slip and no-slip calculations at low Reynolds number, it is possible to find a no-slip calculation which is very similar to the slip calculation when boundary-enhanced viscosity is used.

\subsection{Conclusion}

Once large Re(int) has been reached, the single-gyre model exhibits many interesting behaviors. Through the use of boundary-enhanced viscosity presented in the last chapter, this limit can be studied with western-intensified solutions. In this chapter, some of the interesting effects of the eddies that arise in the case of large $\operatorname{Re}(\mathrm{int})$ have been presented.

The structure of the boundary layer in the main region of eddy activity is novel in this context, although it resembles the boundary layer structure proposed by Edwards and Pedlosky (1998) for transport across the equator. In the region where the Charney boundary-layer solution exists, the effect of the eddies is to widen the region of frictional influence by transporting vorticity to the region of dissipation. In the region where the Charney solution no longer exists or is unstable, however, the primary balance of vorticity in the boundary layer (outside of the frictional sublayer) is between the $\beta$-flux divergence and the eddy flux convergence.

In the quiescent regions where the velocities are small, the single-gyre model at high Reynolds number produces a counter-rotating gyre instead of a Sverdrup balance. These counter-rotating gyres are present in the figures of Holland and Lin (1975), Kamenkovich et al. (1995), and Sheremet et al. (1995), but are significantly weaker than those present here and go uncommented upon in the text of those papers. 
Greatbatch and Nadiga (1999) and Holm and Nadiga (submitted 2002) were able to roughly parameterize these counter-rotating regions with various schemes for homogenization of absolute vorticity. However, the most promising understanding comes by comparison with the analysis of Pedlosky (1965b) of the nonlinear interaction of resonant basin modes. The counter-rotating gyre is nearly inviscid, and the primary balance in the vorticity equation is between the eddy advection and the wind forcing. If the basin is enlarged to have a region without wind forcing, the counter-rotating regions are dynamically similar to those proposed by Pedlosky (1965b) in the windless region. Thus, it seems that an adaptation of Pedlosky (1965b) might yield a good analytic model of the counter-rotating gyre, as it seems that the eddy activity causing these counter-rotating regions is closely related to the resonant basin modes. I believe this to be an excellent topic for further investigation.

The temporal structure of the single-gyre calculations becomes quite complicated as they become less viscous. At Reynolds numbers near 1, the interactions occur between a limited number of periodic motions producing other motions at frequencies which are the sums and differences of the original frequencies. At higher Reynolds number, the spectrum becomes broad, and the peaks become less pronounced as the number of possible interactions increase and secondary and tertiary instability mechanisms without fixed frequency of occurrence appear. In the calculations with a broad spectrum, there is more variance in the total kinetic energy, and it is shared between many modes. Even the low frequencies which would be associated with climate variability are present in the spectra. The qualitative structure of the solution strongly affects the variance of the kinetic energy; the more nonlinear, western-intensified solutions tend to have the largest excursions in kinetic energy relative to the average kinetic energy.

Both the temporal structure and the counter-rotating gyres are significantly affected by the presence of modes of variability similar to the basin modes of oscillation. By changing the dimensions of the basin, it is clear that the frequencies of these modes 
of variability are very close to those of the free, linear basin modes. The absence of basin modes can be achieved by arranging for the basin to be small enough that the highest basin mode frequency (corresponding to the gravest mode here) is lower than the lowest frequency of the boundary current instabilities.

Section 3.6 of this chapter presented the energetics of the single-gyre calculations. The energetics of the single-gyre calculation confirm the hypothesis that the eddies gradually replace the direct effects of friction as Re(int) increases, so long as the frictional sublayer is strong enough. This substitution occurs in the energetics as the production of turbulent kinetic energy from the mean flow replaces the direct frictional dissipation of the mean flow kinetic energy with increasing $\mathrm{Re}$ (int). Interestingly, while the frictional dissipation is almost always a sink of mean flow energy, the turbulent kinetic energy production can be of either sign in different regions (which regions are of which sign is likely to be dependent on the form chosen for the energy production terms). When integrated over the whole basin, however, the turbulent kinetic energy production always transfers energy from the mean flow to the turbulent flow. To a lesser degree, the appearance of counter-rotating gyres also reduces the energy input because in these regions the ocean does work on the atmosphere.

Adding bottom friction also changes the character of the solutions. Primarily, if it is strong, it makes the solution laminar by damping the eddy activity. In particular, because it is not scale-selective it greatly reduces the activity of the basin modes and often removes the counter-rotating gyres. The bottom friction is active in the flux across mean streamlines throughout the basin and not only within a frictional sublayer. Even for small values of $\delta_{S}$, the bottom friction is the primary mode of vorticity transport across the mean streamlines of the recirculation gyre. Its efficient transport of vorticity out of the recirculation gyre can prevent vorticity from being trapped there and reduce the strength of the recirculation dramatically. Interestingly, the lateral frictional sublayer is able avoid the removal crisis when bottom friction is present, a result which will we will return to in section 4.4.4. The removal of vorticity 
from the basin by bottom friction at the slip boundaries is also present, but it is usually a small effect.

Slip boundary conditions greatly reduce the production of eddies near the boundary when compared to no-slip boundary conditions. The reduction seems due to a diminished gradients in the relative vorticity. The reduction even makes it possible for an inertially-dominated solution to be steady. In this respect, the slip boundary conditions seem quite unrealistic in the oceanic context where the boundaries are expected to generate much variability. Using boundary-enhanced viscosity ameliorates this problem somewhat by generating some eddies near the boundary. These eddies are able to flux vorticity toward the boundary and control the circulation as with no-slip boundary conditions, but they require a much smaller Re(bdy) to control the same $\operatorname{Re}($ int $)$.

In conclusion, despite the simplicity of the single-gyre homogeneous ocean model this model becomes quite complicated and revealing as it becomes more inviscid while remaining western-intensified. The presence of strong eddies adds quite a lot to the dynamics. Of course, although this model is greatly simplified, as it is based on the physics which operate in the real ocean, perhaps some of these phenomena are present there. 


\section{Chapter 4}

\section{Adding a Second Gyre}

The results of the preceding chapters demonstrate that eddy transport of vorticity plays a controlling role in setting the circulation strength; the eddies transport vorticity across the streamlines of the mean flow to a frictional sublayer. Marshall (1984) and Harrison and Holland (1981) suggest a different role for eddies in controlling the circulation strength: they could flux vorticity to a second gyre, representing the subpolar gyre, and cancel wind's vorticity input internally without requiring frictional removal. They assert that an eddy flux of vorticity between the gyres reduces the strength of the circulation.

Because this is only one of many theories of control of the circulation strength which require a second gyre, I have conducted experiments adding a second gyre to evaluate these theories. In the introduction, I will briefly review the mechanisms that have been proposed, then add my own mechanism to the list. The results of the numerical calculations with models with a second gyre will then be presented, and then I present a full explanation of the mechanisms and evaluation of the mechanisms using the numerical results.

The work of Lozier and Riser (1990) (who used slip boundary conditions) and Berloff et al. (2002) (who used no-slip boundary conditions) and subsequent work using re-entrant channel idealized jets by Pratt et al. (1995) and Rogerson et al. (1999) 
has shown that most of this eddy transport is not likely to be a simple exchange of fluid parcels (along with their native vorticity) across the jet dividing the gyres. In all of these studies, a vanishingly small mass transport occurs across the jet. Since the transfer needs to be irreversible, the eddies cannot later return to their native gyre unchanged as they would in a wavelike process. They must be able to significantly mix off vorticity before they return. Lagrangian tracers cross the jet semi-permanently approximately of $5 \%$ of the time, and this percentage appears to be approximately correct for oceanographic float observations as well, as shown by Bower and Rossby (1989). These results will be reviewed in section 4.4.1.

Lozier and Riser (1989) and Lozier and Riser (1990) propose a mechanism for their model with slip boundary conditions which allows for vorticity transport without permanent mass transport which they call dissipative meandering. Essentially, this involves a parcel crossing the mean inter-gyre boundary temporarily (as with a passing Rossby wave), then experiencing significant dissipation, and finally returning to its native gyre. By this method, the vorticity is exchanged across the front by an eddy flux, $\overline{\mathbf{u}^{\prime} \zeta^{\prime}}$. In the idealization of Lozier and Riser (1989), this mechanism occurs without breaking the isolines of vorticity; the irreversibility stems from the action of dissipation. They assert that it is the motion of vorticity isolines relative to streamlines by the action of dissipation which is important. They observe in their model that the bulk of the transfer of vorticity occurs in a region very close to the western boundary, consistent with the prominent role of dissipation in their model. Dissipative meandering will be evaluated in section 4.4 .2 .

A different mechanism which requires temporary relocation of the parcels is the temporary exposure of a parcel to the opposing wind forcing. In this mechanism, as in dissipative meandering, a particle with a particular vorticity crosses the mean inter-gyre boundary, its vorticity is changed and then it returns to its native gyre. In this mechanism, however, the parcel's vorticity is changed by the opposing wind forcing instead of dissipation before it returns to its native gyre. The exposure to 
opposing wind forcing will be evaluated in section 4.4.3.

Of course, instead of an eddy flux of vorticity, a mean flux of vorticity can occur. As we will see in section 4.3 , this has an important effect in the case of slip boundary conditions with the wind forcing gyres of unequal strength.

I propose a final and new mechanism for control of the circulation strength in section 4.4.4: change in the modes of instability. This mechanism differs from all of the mechanisms above in that it does not require an inter-gyre vorticity flux. Instead, it relies on a change in the instabilities increasing the efficiency of the flux across mean streamlines to the frictional sublayer. In increasing the efficiency, the eddies formed by the new modes of instability play a role similar to the role of the bottom friction in section 3.7. However, the change in the instabilities may also cause an inter-gyre flux, which occurs with slip boundary conditions. Whether or not there is an inter-gyre flux, the new eddies can aid in controlling the circulation strength. This mechanism differentiates between the single-gyre model and models with a second gyre, but it is present whether or not there is opposing wind forcing; it relies only on the removal of the northern boundary at the line of zero wind-stress curl.

Which mechanism is preferred for the removal of the wind's vorticity input? Is there an inter-gyre flux of vorticity, or is there an eddy flux of vorticity to a frictional sublayer? In the preceding chapters, it is demonstrated that the ultimate frictional removal of vorticity had a feedback on the eddy fluxes; if there is boundary-enhanced viscosity which mechanism is preferred? What role do the boundary conditions play? Obviously, re-evaluating the previous studies by adding a second gyre to the models created for the work of the preceding chapters is of interest.

Marshall (1984) and Harrison and Holland (1981) used a double-gyre wind forcing which was exactly antisymmetric about the middle of the basin. Thus, this wind forcing had no net circulation input to the basin. Because the ultimate removal of vorticity from the basin plays a critical role in preceding chapters, an additional model is studied here to explore the disposal of vorticity in a multiple gyre model. This new 
model is similar to the double-gyre but with a weaker subpolar gyre so that the wind circulation input is not exactly zero. The three models, single-gyre, two-gyre and double-gyre, are presented in section 4.1 .

In section 4.2 , calculations with each wind forcing are compared in models with no-slip boundary conditions. The circulation strength is reduced with the addition of a second gyre. However, the reduction in circulation is not because of an inter-gyre flux of vorticity.

In section 4.3 , calculations with each wind forcing are compared with slip boundary conditions. These boundary conditions are closer to those used by Marshall (1984) and Harrison and Holland (1981) than no-slip, and the double-gyre calculations show a substantial eddy flux of vorticity from one gyre to the other. As in Lozier and Riser (1990), the vorticity exchange occurs in a region very near the boundary. However, when the anti-symmetry of the double-gyre wind forcing is not present, the eddies are no longer primarily responsible for the flux into regions of opposing wind forcing. When the gyres are of unequal strength, the subtropical western boundary current overshoots, and it is this mean flow which redistributes the vorticity input by the wind forcing.

To determine the cause of the reduction of circulation strength in sections 4.2 and 4.3 , section 4.4 reviews the hypothetical mechanisms mentioned above in turn. In the no-slip case, as there is very little inter-gyre vorticity flux, it must be the new modes of instability (particularly instabilities of the jet) that are responsible for the change in the circulation strength. The eddies formed by these instabilities are able to vastly increase the efficiency of vorticity transport to the frictional sublayer. With slip boundary conditions, the inter-gyre flux is important as in Marshall (1984) and Lozier and Riser (1990), but the inter-gyre flux may not be an eddy flux. When the wind forcing is exactly antisymmetric, dissipative meandering is quite important resulting in an important inter-gyre eddy flux of vorticity. When the wind forcing is not exactly antisymmetric, the subtropical gyre dominates the weaker subpolar gyre 
resulting in an important inter-gyre mean flux of vorticity.

In the last section, the role of boundary-enhanced viscosity in models with multiple gyres is reviewed. It is shown that at sufficient Reynolds number, even in the presence of the efficient sinuous modes, a two-gyre solution can become inertially-dominated. By using boundary-enhanced viscosity this model can be controlled just as the results of Chapter 2 demonstrate for the single-gyre. Also, it is noted that when boundaryenhanced viscosity is used in slip calculations, they have less of an overshoot, a small eddy flux to a frictional sublayer, and the direct removal of vorticity by the mean flow is larger and the inter-gyre vorticity flux is smaller.

\subsection{The Single-gyre, Double-gyre and Two-gyre Models}

In this chapter, three classes of wind forcing and basin arrangements are used: the single-gyre as in the preceding chapters, the antisymmetric double-gyre, and the asymmetric two-gyre forcing.

The single-gyre model is exactly the one used in the preceding chapters. It is a square domain with $x$ and $y$ ranging from zero to one with a dimensionless vorticity input from the wind of

$$
w_{E *} / W_{E}=-\sin (\pi y)
$$

In the double-gyre forcing, the subtropical wind forcing extends over the $x$ region from 0 to 1 and the $y$ region from 0 to 1 as in the single-gyre. The vorticity input is identical to that in the single-gyre over this region. The subpolar gyre of the doublegyre model extends over the $x$ region from 0 to 1 and the $y$ region from 1 to 2 . The subpolar gyre has equal and opposite vorticity forcing as the subtropical gyre. The wind forcing in the double-gyre model is the same as that used in Marshall (1984) (other than in nomenclature). 
Note that the vorticity input is exactly antisymmetric about the $\mathrm{y}=1$ line in the double-gyre model. I will return to discuss this symmetry many times, because the solutions of the double-gyre are strongly affected by it.

The third model is a two-gyre model, which also has subtropical and sub-polar gyres but they are not exactly antisymmetric. Instead, as in the real ocean, the sub-polar gyre is smaller than the subtropical and its wind forcing is weaker. The forcing in this model was made to resemble the zonally-averaged circulation input found for a realistic model of the North Pacific (Huang and Chao (Submitted 2001)). A polynomial expansion of the forcing was chosen so that there would be no rough transitions in forcing or its derivatives. The amplitude of this polynomial was then normalized so that the circulation input to the subtropical gyre was the same as in the other two models, and the northward extent of the basin was chosen so that the zero wind-stress curl line lies at $y=1$ as in the other models. This analysis resulted in the following quintic polynomial approximation for the wind stress:

$$
w_{E}=-\frac{2}{\pi}\left(1+\frac{4 y}{L_{y}}\right) \frac{\frac{5 y^{4}}{L_{y}^{4}}-\frac{16 y^{3}}{L_{y}^{3}}+\frac{16 y^{2}}{L_{y}^{2}}-\frac{5 y}{L_{y}}}{\frac{10}{3 L_{y}^{5}}-\frac{59}{5 L_{y}^{4}}+\frac{12}{L_{y}^{3}}-\frac{4}{3 L_{y}^{2}}-\frac{5}{2 L_{y}}} .
$$

The basin dimension in the zonal direction was chosen to be the dimensional unit, so the zonal spread remains 0 to 1 . The meridional basin dimension of the twogyre case, dubbed $L_{y}$, was chosen so that the zero wind forcing line matched that of the double-gyre and single-gyre calculations. This choice resulted in the northern boundary being located at $L_{y}=(11+\sqrt{21}) / 10$ which is approximately 1.56 .

Thus, in all three models, the subtropical wind forcing extends over the $x$ region from 0 to 1 and the $y$ region from 0 to 1 as in the single-gyre. The subtropical gyre was kept constant in terms of total vorticity input and northward extent of negative wind forcing so that direct numerical comparisons could be made between the models. The vorticity input of the wind in the three models is shown in figure 4-1. 


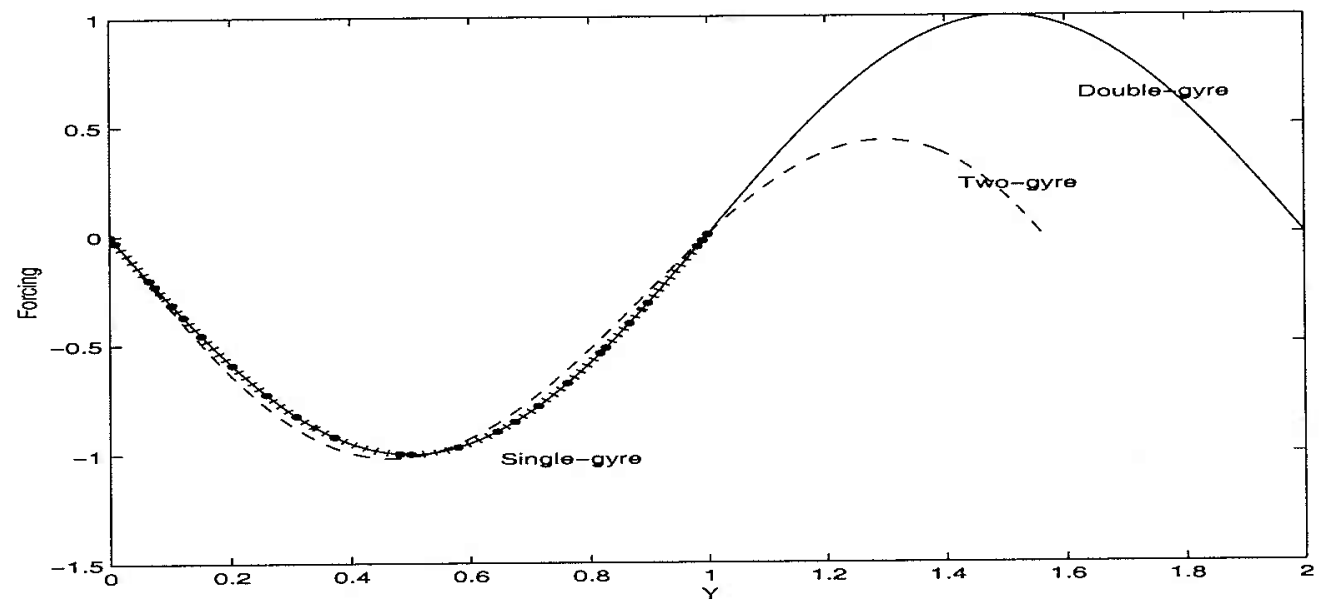

Figure 4-1: Comparison of the vorticity input by the wind in the three models. Double-gyre forcing is shown as a solid line, two-gyre forcing is shown as a dashed line, and single-gyre forcing is shown as a slashed and dotted line (which overlays the double-gyre forcing).

\subsection{Results with No-Slip Boundary Conditions}

In this section, the results of the single-gyre calculation with no-slip boundary conditions on the eastern and western boundaries are compared to the two-gyre and double-gyre calculations with the same boundary conditions.

The results from a single, double, and two-gyre calculation, each with $\operatorname{Re}(\mathrm{bdy})=5$, $\operatorname{Re}($ int $)=5$, are shown in figure 4-2. Obviously, the circulation is much stronger in the single-gyre calculation. The two-gyre and double-gyre calculations have much weaker circulations. They have similar magnitudes to each other in the subtropical gyre. Importantly, the recirculation gyre is almost completely missing in the double and two-gyre calculations, and what is remaining of it is stretched along the meridional direction.

The eddy flux of vorticity across the streamline dividing the gyres is also given in figure 4-2. In both the double-gyre and the two-gyre cases, it is extremely small, much smaller than the flux into the counter-rotating region, for example. Given the inaccuracies due to numerical error and errors in averaging, this flux is too small to be 


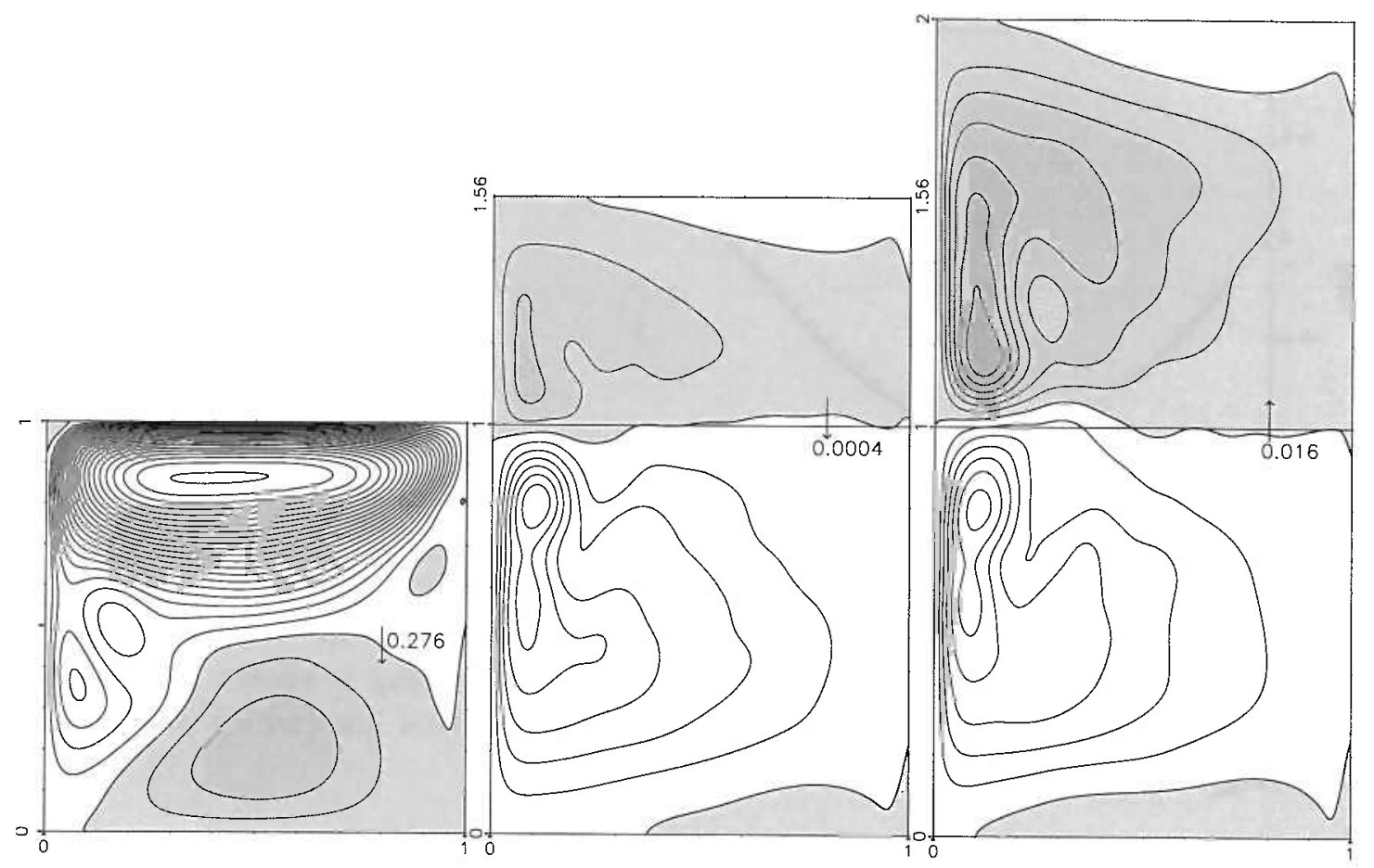

Figure 4-2: Contours of the time-mean streamfunction for different basins and wind forcing with $\operatorname{Re}(\mathrm{bdy})=5, \operatorname{Re}(\mathrm{int})=5$, and no-slip boundary conditions. The contour interval is 0.2 , regions of negative streamfunction are shaded. The arrows on the figure give the eddy flux of vorticity across that zero contour of psi. (The total vorticity input from the wind to the subtropical gyre is 0.637 .) The contour interval is 0.2 .

distinguishable from zero. In fact, the direction of the vorticity exchange is different in the double-gyre and the two-gyre models.

Figure 4-4 shows the single, double, and two-gyre calculations with boundaryenhanced viscosity. The results are surprisingly similar to those in figure 4-2 for the double and two-gyre calculations. This is because in figure 4-2 and figure 4-4, the calculations have a nearly Sverdrup interior (See figure 4-3). Thus, although the boundary-enhanced viscosity changes the boundary current and recirculation somewhat, because the interior is nearly Sverdrup, there are few differences between the two-gyre and double-gyre calculations with and without boundary-enhanced viscosity. The inter-gyre vorticity fluxes remain very small in the boundary-enhanced viscosity 


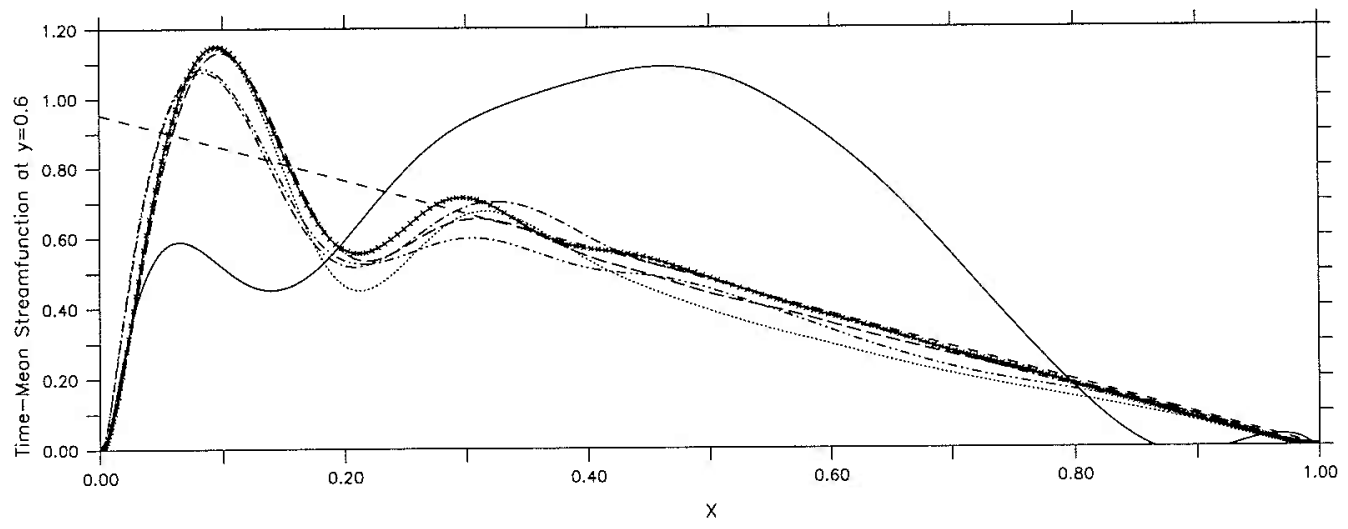

Figure 4-3: Comparison of the time-mean streamfunction at $y=0.6$. The following calculations are shown: no-slip, $\operatorname{Re}($ bdy $)=\operatorname{Re}($ int $)=5$ single-gyre (solid), twogyre (dot-dot-dashed), and double-gyre (dot-dashed); and no-slip, $\operatorname{Re}($ bdy) $=0.25$, $\operatorname{Re}($ int $)=5$ single-gyre (dotted), two-gyre (crosses), and double-gyre (long-dashed). Note how only the $\operatorname{Re}(b d y)=\operatorname{Re}(i n t)=5$ single-gyre (solid) calculation, which is inertially-dominated diverges significantly from the Sverdrup solution (dashed) in the interior. Within the recirculation $(x \approx 0.1$ to 0.3$)$, small differences between the other solutions are apparent.

calculations. I will return to discuss the necessity and results of using boundaryenhanced viscosity at higher interior Reynolds number in section 4.5.

So, at the same Reynolds number the two and double-gyre circulations with no-slip boundary conditions have nearly the same circulation strength as the linear Munk solution, although the single-gyre calculations are significantly stronger than the Munk solution. This occurs without a substantial inter-gyre eddy flux of vorticity.

\subsubsection{Frictional and Eddy Fluxes}

It has been proposed by Marshall (1984) and Harrison and Holland (1981) that in time-dependent, high Reynolds number calculations of the barotropic double-gyre circulation, an eddy flux of vorticity exchanges vorticity from gyre to gyre. If this 


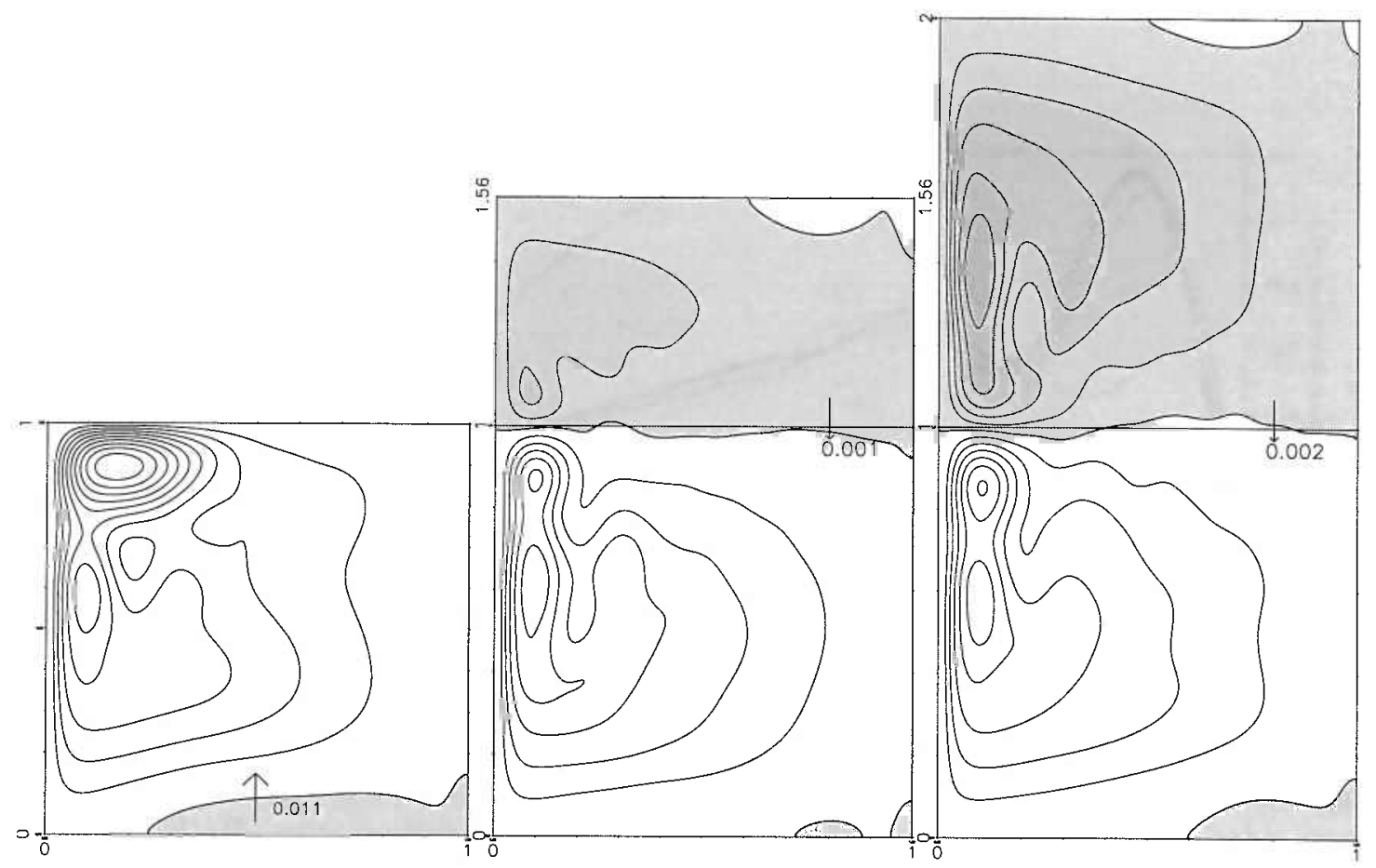

Figure 4-4: As in figure 4-2, except for the $\operatorname{Re}(\mathrm{bdy})=0.25, \operatorname{Re}(\mathrm{int})=5$, no-slip calculations.

occurs, since the sense of rotation of the two gyres is opposite, the circulation could be controlled, or at least the work needed to be performed by frictional removal is decreased.

To show the inter-gyre eddy fluxes, Marshall (1984) removed one part of the rotational fluxes to demonstrate that the remaining, primarily divergent, flux was across the inter-gyre boundary. The separation used depended critically on there being a functional relationship between $\bar{\psi}$ and $\bar{\zeta}$. Marshall (1984) chooses super-slip boundary conditions $\left(\frac{\partial \zeta}{\partial n}=0\right)$ with the goal of producing a functional relationship between $\bar{\psi}$ and $\bar{\zeta}$. He argues that using no-slip or slip boundary conditions unrealistically reduce the advection of vorticity along the boundary. Using super-slip boundary conditions, he is able to show that there is an approximate functional relationship between $\bar{\psi}$ and $\bar{\zeta}$, at least in a limited region of the basin. Here, as in Roberts and Marshall 
(2000), we are using no-slip boundary conditions and there is no single-valued functional relationship between $\bar{\psi}$ and $\bar{\zeta}$. No functional relationship exists when using slip boundary conditions either. Alternate methods for separating the fluxes into divergent and rotational parts are not unique and therefore inadequate to demonstrate inter-gyre fluxes, as pointed out in Fox-Kemper et al. (2003).

Therefore, the inter-gyre fluxes are analyzed here as budgets from one region to another. When the divergence of the flux is integrated over a closed region, the flux out of that region is obtained. This result was presented in a simpler context in equation 2.15 and 2.16. Here, as in the preceding chapters, we will use mean streamlines as the boundaries of the regions of integration. By choosing these regions, only the frictional fluxes and eddy fluxes are present because the mean flux and the $\beta$-flux cancel, as shown in equation 2.15.

The inter-gyre flux of vorticity in the no-slip calculations performed here seems to be negligible. In fact, it is typically the smallest in the two-gyre and double-gyre calculations when compared to all of the other fluxes, eddy and frictional. In figures 42 and 4-4, no-slip calculations in different basins are compared side-by-side. In all of these calculations, the inter-gyre flux of vorticity never exceeds $0.2 \%$ of the vorticity input to the subtropical gyre (the total input is approximately 0.637 ). In fact, even the sign of the eddy flux appears to be random or at least indeterminate. In any case, it is highly improbable that such a small flux controls the circulation strength, when a tiny percentage change in the frictional removal would produce a much larger effect.

The result is changed little when an enhanced frictional sublayer is used as in figure 4-4. The preceding chapters demonstrate that in single-gyre calculations the boundary-enhanced viscosity is needed to control the circulation, as the friction near the boundary ultimately removes the vorticity. In the multiple-gyre calculations, there is obviously a mechanism at work which reduces the circulation, yet it does not require the boundary-enhanced viscosity at this $\operatorname{Re}(\mathrm{int})$, nor is the inter-gyre eddy 
flux responsible.

In the discussion of section 3.7, I argued that the removal crisis was averted when bottom friction was present because the bottom friction prevented a response to the increased vorticity gradient near the boundary. The results in figures 4-2 and 4-4 seem to indicate that something introduced with the second gyre may also prevent the removal crisis response.

In figures 4-5 and 4-6, the eddy fluxes of vorticity are compared with the frictional fluxes of vorticity across a few important streamlines. In agreement with the assertions of the previous chapters, there is a large divergence in the flux between the $\bar{\psi}=0$ and $\bar{\psi} \approx 0.25$ mean streamlines. This is indicated in figures $4-5$ and $4-6$ by a large difference in the eddy flux across the $\psi=0$ streamline demarcating the counter-rotating gyre and the $\bar{\psi}=0.25$ streamline. This vorticity dropped off by this eddy flux divergence is picked up by a large frictional flux convergence and transported through the basin boundary. The magnitude of the frictional fluxes across the interior mean streamlines decreases rapidly from the flux through the boundary, indicating a frictional flux convergence contained in a region close to the boundary.

Thus, the mechanism of the preceding chapters is represented here. The transfer between frictional dissipation and eddy fluxes is revealed in these figures as an increase in the eddy flux magnitude and a decrease in the frictional flux magnitude as one considers streamlines farther from the boundary. For example, in figure 4-6, where the eddy flux across the $\bar{\psi}=0.1$ streamline is only 0.067 and the flux across the $\bar{\psi}=0.25$ streamline is 0.218 . A corresponding decrease in the frictional flux magnitude confirms that the vorticity is transferred from the frictional fluxes to the eddy fluxes in this region near the boundary.

For almost all of the interior streamlines the frictional fluxes are much smaller than the eddy fluxes. The notable exception is the $\psi=0.1$ mean streamline, which is included because it indicates that the flux across streamlines which enter the frictional sublayer is largely frictional. 

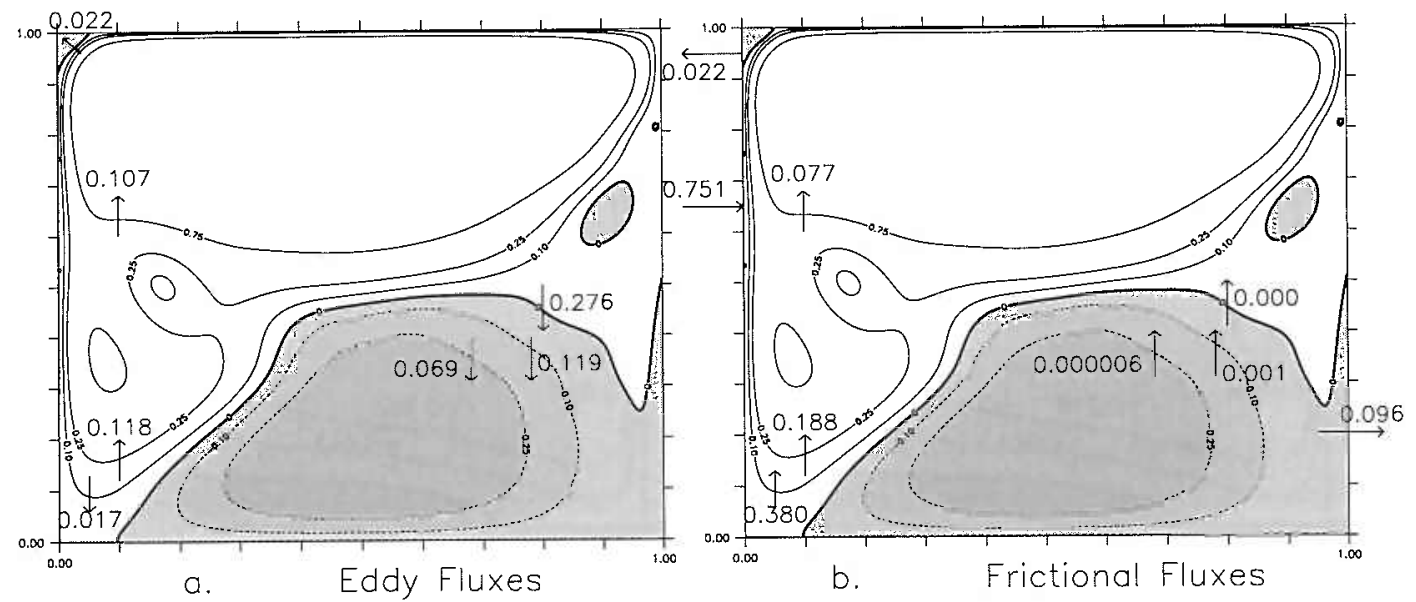

Figure 4-5: Best estimates of (a) time-mean eddy fluxes of vorticity across chosen mean streamlines (those shown with thick lines $\bar{\psi}=$ $\{-0.75,-0.25,-0.1,0,0.1,0.25,0.75\}$ ) and (b) time-mean frictional fluxes of vorticity across the same streamlines. The frictional fluxes through the boundary may not represent a simply connected region where counter-rotating gyres are present (e.g., it may include flux through eastern, northern, and western boundaries added together). The regions are divided by the intersection of the streamline where $\bar{\psi}=0$ and the boundary. The calculation shown is the no-slip, single-gyre, $\operatorname{Re}(b d y)=5$, $\operatorname{Re}($ int $)=5$ calculation

In interpreting the results of figures like figure 4-5, it is important to remember that some of the convergence present in the frictional and eddy fluxes is used in canceling the wind input. For example, the difference between the 0.118 flux across the $\bar{\psi}=0.25$ streamline and the and 0.107 flux across the $\bar{\psi}=0.75$ streamline is most likely being used to cancel the wind input between those streamlines. Obviously, the 0.107 flux into the closed region of the recirculation gyre is entirely canceled by the wind forcing there.

In agreement with the results of the previous chapters, the counter-rotating gyres in figures 4-5 and 4-6 are primarily eddy-driven and relatively inviscid. This is shown in these figures by the significantly larger eddy fluxes across their bounding streamline than the frictional fluxes into the counter-rotating region. 

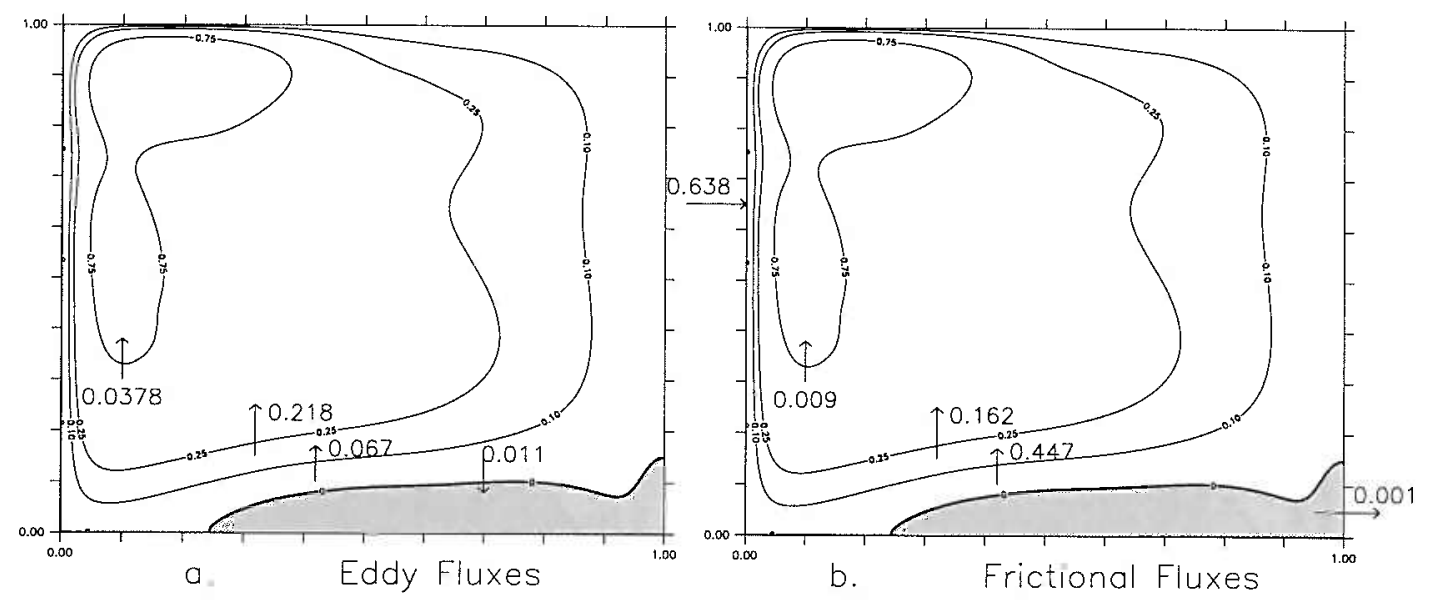

Figure 4-6: Time-mean eddy fluxes and frictional fluxes as in figure 4-5 except the calculation shown is the no-slip, single-gyre, $\operatorname{Re}(\mathrm{bdy})=0.25, \operatorname{Re}($ int $)=5$ calculation. Note that only selected streamlines are displayed.

Figure 4-7 shows the eddy and frictional fluxes across mean streamlines in no-slip, two-gyre calculations with constant viscosity. In this calculations, we see essentially the same mechanism of vorticity removal as in the single-gyre calculations. There is a large eddy flux divergence between the first few interior streamlines in the subtropical gyre. This vorticity is removed by frictional fluxes through the boundary. The increase in eddy flux between the $\bar{\psi}=0.1$ streamline and the $\bar{\psi}=0.25$ along with a corresponding decrease in frictional fluxes indicates the presence of the boundary-layer processes described in the preceding chapters. However, note by comparing figure 4-2 and 4-4 that the boundary-enhanced viscosity does not have nearly as much of an effect on the two-gyre calculations as it does in the single-gyre calculations.

Figure 4-8 shows the eddy and frictional fluxes across mean streamlines in twogyre calculations with constant viscosity. Just as in the single-gyre and two-gyre calculations, eddy fluxes to a frictional sublayer are responsible for the bulk of the vorticity removal from the basin.

Why there is such a striking difference between figure 4-5 and figures 4-7 and 

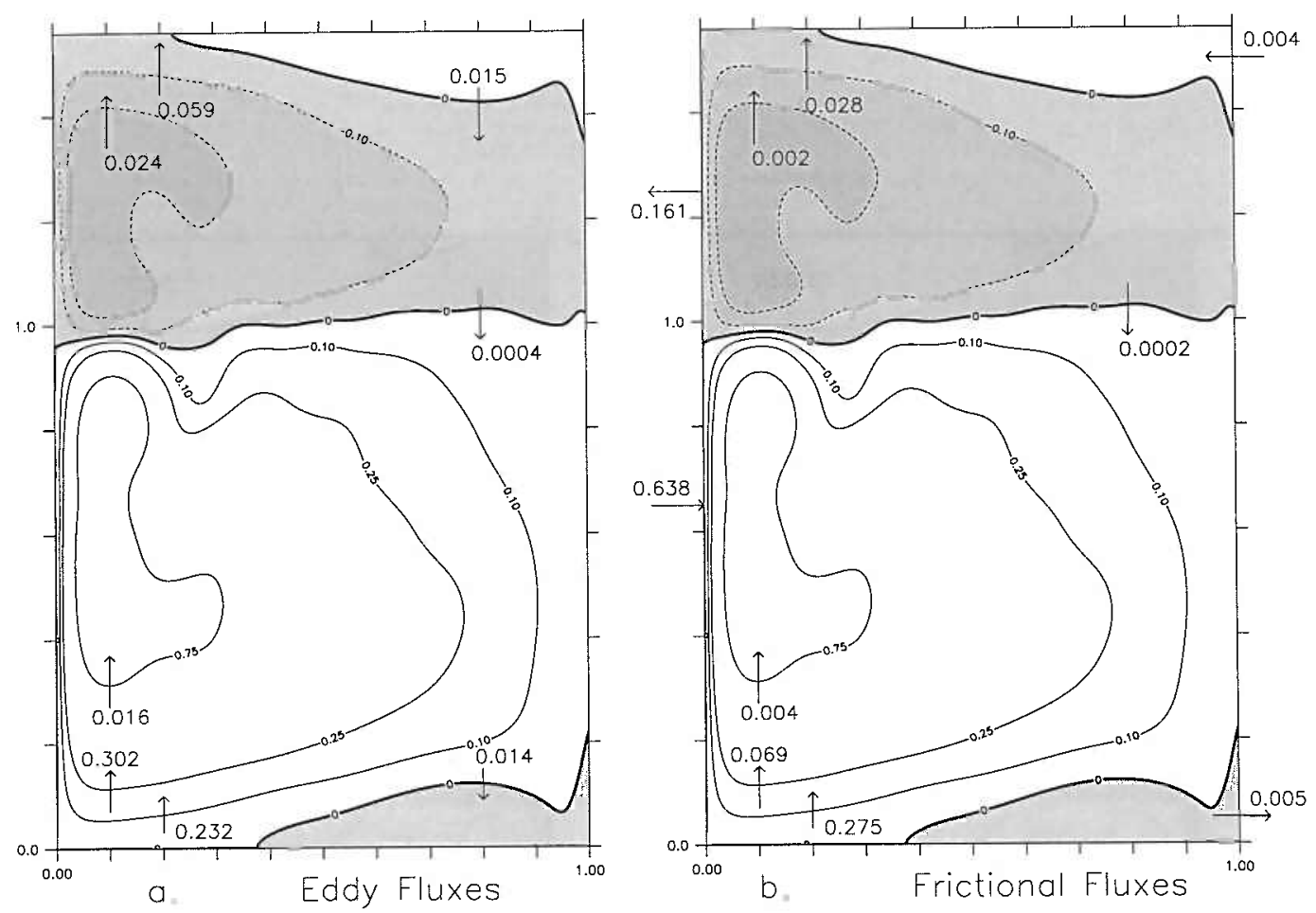

Figure 4-7: Time-mean eddy fluxes and frictional fluxes as in figure 4-5 except the calculation shown is the no-slip, two-gyre, $\operatorname{Re}(\mathrm{bdy})=5, \operatorname{Re}(\mathrm{int})=5$ calculation. Note that only selected streamlines are displayed.

4-8? The preceding chapters show that a boundary-enhanced viscosity is required to make the frictional sublayer capable of smoothly removing the vorticity input from the basin. Here, even though the viscosity is constant through the basin, the eddy flux of vorticity to the frictional sublayer is capable of controlling the circulation. Apparently, something has changed either in the nature of the frictional sublayer, the mean flow, or the eddy fluxes to the frictional sublayer which allows vorticity to be removed from the basin without a removal crisis and inertial-domination. It is not an eddy flux of vorticity between the gyres that controls the circulation in the no-slip calculations. 

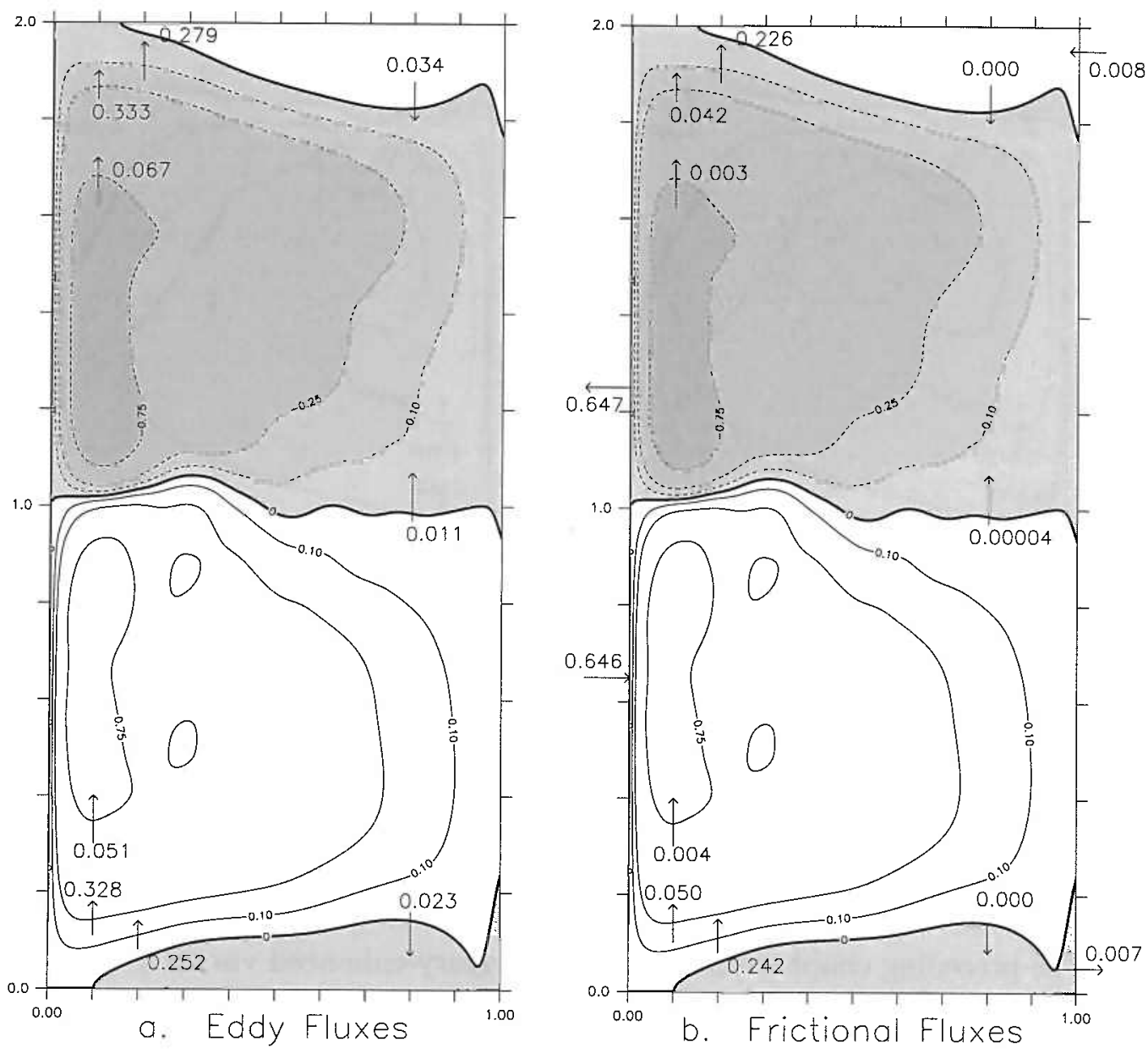

Figure 4-8: Time-mean eddy fluxes and frictional fluxes as in figure 4-5 except the calculation shown is the no-slip, double-gyre, $\operatorname{Re}(b d y)=5, \operatorname{Re}($ int $)=5$ calculation. Note that only selected streamlines are displayed. 


\section{Errors in Flux Estimates}

The fluxes shown in the figures 4-5 and 4-6 and similar figures appearing later are described in the caption as 'best estimates' because there is more than one approach used in their calculation. Wherever possible, and always in the case of eddy fluxes, the flux divergence is integrated over the region enclosed by the streamline to determine the cross-streamline flux. This minimizes the effect of errors in the eddy flux. For the frictional flux, the errors result from calculating many derivatives of the mean flow, experience has shown that integrating the flux divergence over a large area is useful in evaluating those terms as well.

Because the frictional fluxes do not vanish at the boundaries other approaches are necessary in conjunction with integration of the divergences. Wherever possible, larger areas connected to the boundary are used as areas of integration for the frictional flux divergences. This allows the construction of budgets which constrain the frictional flux estimates. In some regions, it remains necessary to directly evaluate the spatial distribution of $\delta_{M}^{3} \nabla \bar{\zeta}$ to close the budgets. For example, the inter-gyre frictional flux is estimated as the average of $\delta_{M}^{3} \frac{\partial \bar{\zeta}}{\partial y}$ over a small region enclosing the inter-gyre boundary. The result is observed to be relatively insensitive to the location of the inter-gyre boundary and the region of averaging. The $\delta_{M}^{3} \frac{\partial \bar{\zeta}}{\partial x}$ component is small and nearly perpendicular to the inter-gyre boundary in this region which also aids the estimation. Dividing the flux out of the counter-rotating gyres into a component through the boundary and another into the interior is also necessarily estimated by examining the spatial distribution of $\delta_{M}^{3} \nabla \bar{\zeta}$.

The flux determination method is complicated enough to present substantial difficulties in propagating the error estimates through the calculation. Also, errors are surely present that are related to whether the model was run sufficiently long enough to get an accurate average. It is easy to find an accurate average for the streamfunction, but for the relative vorticity, eddy fluxes, and friction fluxes it is quite difficult.

I have determined that the errors made in determining the fluxes by the 'best 
estimate' method are not significant when compared to errors in the averaging. Their size can be estimated from the variability in figures similar to figure 2-9.

The magnitude of the averaging errors is estimated by calculating the averages over only part of the time interval used in generating the figures like figure 4-5. An example calculation averaging over a reduced time interval corresponding to figure 4-8 is shown in 4-9. It is clear from figure 4-9 that most of the fluxes are statistically stable, especially those of large amplitude or those which result from integration over a large region. The smaller fluxes are known to within an order of magnitude at least, with the exception of the frictional inter-gyre flux which is very small.

It is difficult to directly estimate the errors in the flux estimates, and so the the fluxes have been given no explicit errors. Instead the error estimates have been used to choose of the number of digits shown. Although the protocol for significant digits is not used strictly as budgeting is necessary in the error estimation process, the number of digits given for each flux should be the correct number, or occasionally one more digit is present.

In summary, the vorticity flux estimates for no-slip calculations presented here differ dramatically from previous attempts to diagnose the inter-gyre vorticity flux, all of which use different boundary conditions. There is only a vanishingly small inter-gyre flux of vorticity, although the eddy fluxes are large and play an important role in transporting vorticity to the frictional sublayer. As in preceding chapters, the boundary current in the exit region is of the type described in section 3.1 , and throughout the interior the flux across mean streamlines is dominated by the eddy fluxes. Despite the lack of an inter-gyre flux of vorticity, the circulation strength of the subtropical gyre is significantly reduced when a second gyre is added, and at the same time, sensitivity to enhancing the boundary viscosity is reduced. 

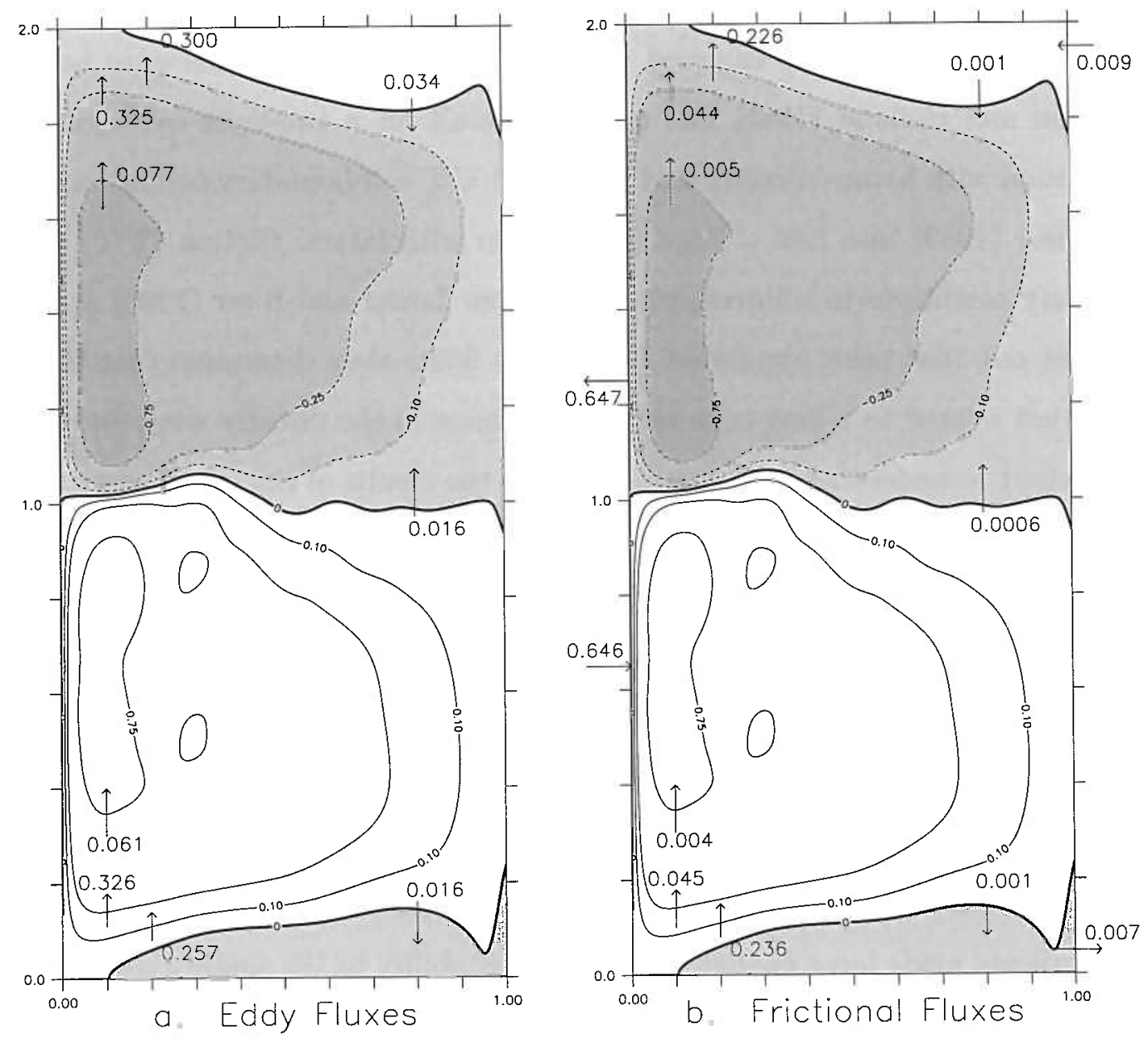

Figure 4-9: Estimates of (a) eddy fluxes of vorticity across chosen mean streamlines, and (b) frictional fluxes of vorticity across the same streamlines. This figure is to be compared to figure 4-8 to estimate magnitude of errors, as in the calculation shown here the time average is taken only over the second half of the interval used in the average for figure 4-8. 


\subsection{Results with Slip Boundary Conditions}

Marshall (1984) uses bottom friction and hyper-viscosity $\left(\nabla^{4} \zeta\right.$ in the vorticity equation) with frictional boundary conditions $\frac{\partial \zeta}{\partial n}=0$ and $\frac{\partial^{3}}{\partial n^{3}} \zeta=0$. He finds that in his calculation of the double-gyre, 0.563 out of an integrated vorticity input of 0.648 to the subtropical gyre is canceled by eddy fluxes across the inter-gyre boundary. Harrison and Holland (1981) find a similar result for a two-layer quasigeostrophic calculation with hyper-viscosity and $\zeta=0$ and $\nabla^{2} \zeta=0$ boundary conditions. Lozier and Riser (1989) also find a large cancellation with lateral friction $\left(\nabla^{2} \zeta\right)$ and slip boundary conditions in a three-layer calculation. Lozier and Riser (1990) are careful to point out that using simulated Lagrangian floats they determine that this eddy flux is not related to a long-term exchange of mass in the directly wind-driven layer. In an effort to understand the contradiction in the results of the previous section and these results, I conducted calculations of the double-gyre and the two-gyre basins with slip boundary conditions.

Recall that in the case of the single-gyre with slip boundary conditions (section 3.8), the eddy fluxes were significantly smaller or nonexistent when compared to the no-slip calculations. Apparently, it is very difficult to generate eddies at the boundary when slip boundary conditions are used because the vorticity gradients are too weak for instabilities to form. Only by using boundary-enhanced viscosity were the gradients made large enough to produce instability in the single-gyre, slip calculations. The single-gyre calculation without boundary-enhanced viscosity was steady, and its lack of eddies lead it to be hundreds of times stronger than the calculation with boundary-enhanced viscosity.

Unlike the double-gyre with no-slip boundary conditions, in the double-gyre with constant viscosity and slip boundary conditions the inter-gyre eddy fluxes are primarily responsible for removing the vorticity. In a calculation with $\operatorname{Re}(b d y)=\operatorname{Re}(\mathrm{int})=2.5$, the eddies dispose of 0.33 out of 0.64 of the wind's vorticity input to each gyre. In a calculation with $\operatorname{Re}(\mathrm{bdy})=\operatorname{Re}(\mathrm{int})=5$, the eddies dispose of 0.56 out of 0.64 of the 

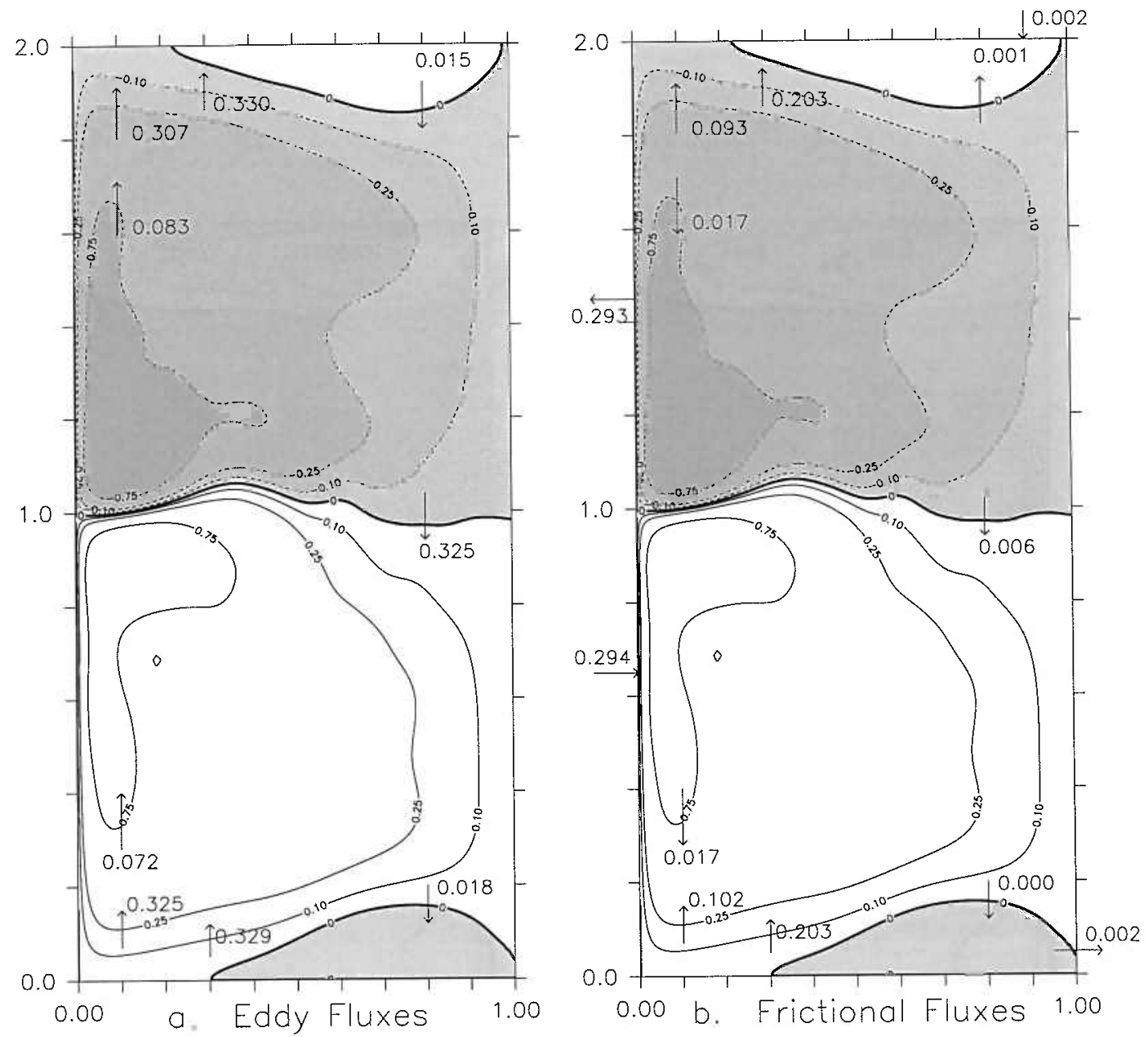

Figure 4-10: As in figure 4-5 except the calculation shown is the slip, double-gyre, $\operatorname{Re}(\mathrm{bdy})=2.5, \operatorname{Re}(\mathrm{int})=2.5$ calculation. Note that only selected streamlines are displayed.

wind's vorticity input to each gyre. The remaining portion was removed by friction. These results are shown in figure 4-10 and 4-11.

In figure 4-10 and 4-11, the eddy fluxes across the streamlines shown do not increase significantly or they decrease with distance from the boundary. As an increase is the clear indicator of the eddy flux to the frictional sublayer, the mechanism of the 

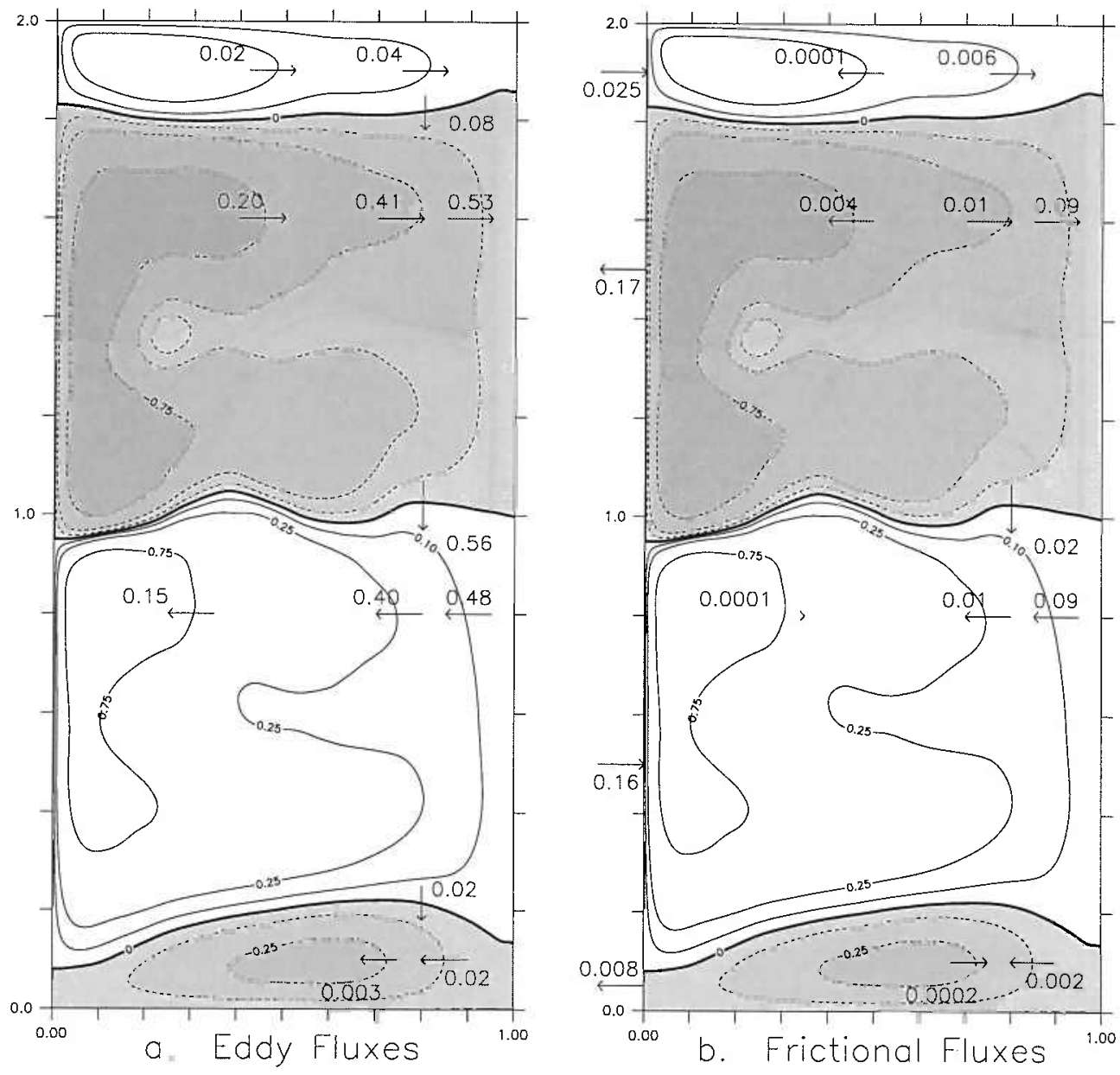

Figure 4-11: As in figure 4-5 except the calculation shown is the slip, double-gyre, $\operatorname{Re}(b d y)=5, \operatorname{Re}($ int $)=5$ calculation. Note that only selected streamlines are displayed. 
preceding chapters appears not to be particularly effective in these calculations. The eddy fluxes and friction fluxes seem to work independently to balance the wind input. The frictional flux removes the vorticity input directly from the mean streamlines that pass close enough to the boundary, and the eddy flux transports vorticity from gyre to gyre, canceling wind input in both regions.

When boundary-enhanced viscosity is used in a double-gyre, slip calculation (figure 4-12), the eddy fluxes perform both an inter-gyre flux (of 0.167 units) and a flux from the frictional sublayer (of approximately 0.02 units). In this case the direct action of friction on the mean flow entering the frictional sublayer is the most important contributor to the removal of vorticity. For the streamlines which do not pass through the frictional region, the eddy flux emanates from within these streamlines and goes primarily into the other gyre where it is removed primarily by dissipative meandering. Apparently, because the viscosity near the boundary is larger here than in the calculation shown in figure 4-11, more of the vorticity input is removed directly by friction in figure 4-12 than in figure 4-11 where most of the vorticity is removed by dissipative meandering.

The frictional dominance of figure 4-12 is consistent with the discussion of section 3.8, where it is pointed out that slip boundary conditions do not easily produce eddies near the boundary. The frictional fluxes also dominate the single-gyre, slip calculation with boundary-enhanced viscosity (as in figure 3-27a). Thus, the eddy flux of vorticity to the frictional sublayer is not particularly effective with slip conditions, although it is somewhat more effective with boundary-enhanced viscosity than with constant viscosity.

However, where eddies are completely absent (as in figure 3-27b) the circulation must gain enormous strength to remove the vorticity from the innermost streamlines frictionally with low viscosity. So, although there are few eddies in the slip calculations of figure 3-27a, they serve an important role in removing vorticity from the innermost streamlines. In the single-gyre case, the eddies flux this vorticity to the frictional 

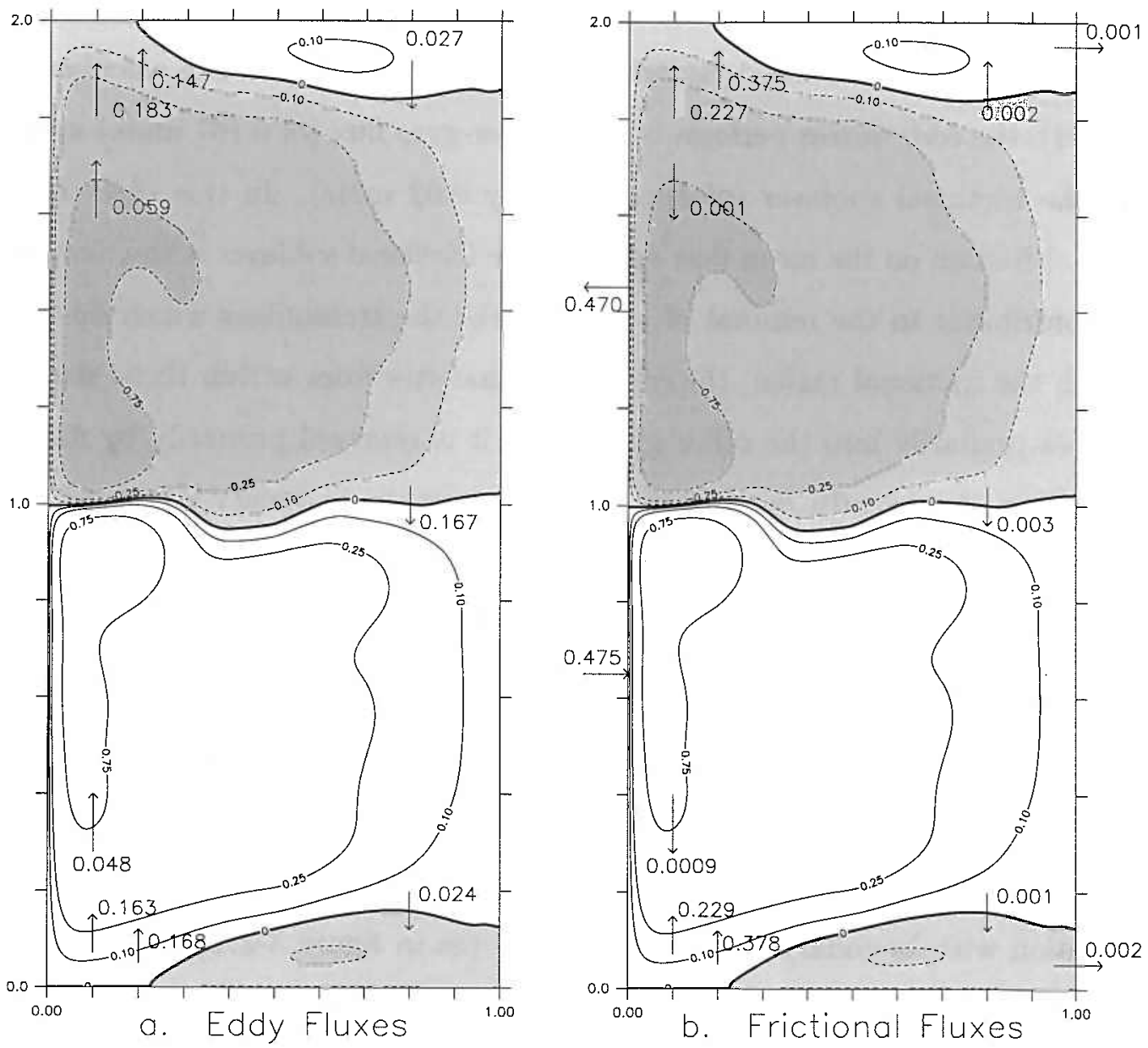

Figure 4-12: As in figure 4-5 except the calculation shown is the slip, double-gyre, $\operatorname{Re}(\mathrm{bdy})=0.25, \operatorname{Re}(\mathrm{int})=5$ calculation. Note that only selected streamlines are displayed. 
sublayer. In the double-gyre case, the eddies flux this vorticity primarily to the other gyre, and to a lesser extent to the frictional sublayer.

A weakness of using a barotropic model for these calculations is the lack of eddy generation at a slippery boundary. As the only mechanism for instability is shear instability or frictional instabilities, and as the slip boundary condition produces very little shear in the boundary layer, there are no eddies formed in the interaction with the boundary. However, it is highly unlikely that this would be true of a real oceanic boundary. The generation of eddies by rough topography is well-known, and more esoteric interactions with a sloping boundary also produce significant eddy activity (Hallberg and Rhines (2000) and Becker and Salmon (1997)). Thus, it seems that the use of a slip boundary condition in a barotropic model is likely to produce too few eddies at the boundary. The results with slip boundary conditions are therefore biased against the interaction of the boundary with the eddy field.

As there are few eddies produced at the boundary, the remaining region for formation of eddies, the jet, dominates the production of eddies in the double-gyre, slip calculation. These eddies produce a large inter-gyre flux of vorticity. Note, however, that the double-gyre is singularly suited to favor inter-gyre eddy fluxes of vorticity rather than other adjustments to control the circulation strength. Given the exact antisymmetry of the wind forcing, over a sufficiently long integration and with sufficiently energetic eddies, it is implausible that the inter-gyre boundary will not lie precisely halfway along the north-south dimension of the basin. Even if the solution were to oscillate between two different, but antisymmetric, quasi-stable solutions, the resulting analysis of the eddy fluxes would diagnose the transitions as inter-gyre eddy fluxes.

When the exact forcing antisymmetry is broken in the two-gyre, slip calculation a very different result arises. In this case, the stronger, subtropical gyre's western boundary current overshoots the zero wind stress curl line and completely dominates the subpolar gyre's boundary current (see figure 4-13). When compared to the double- 

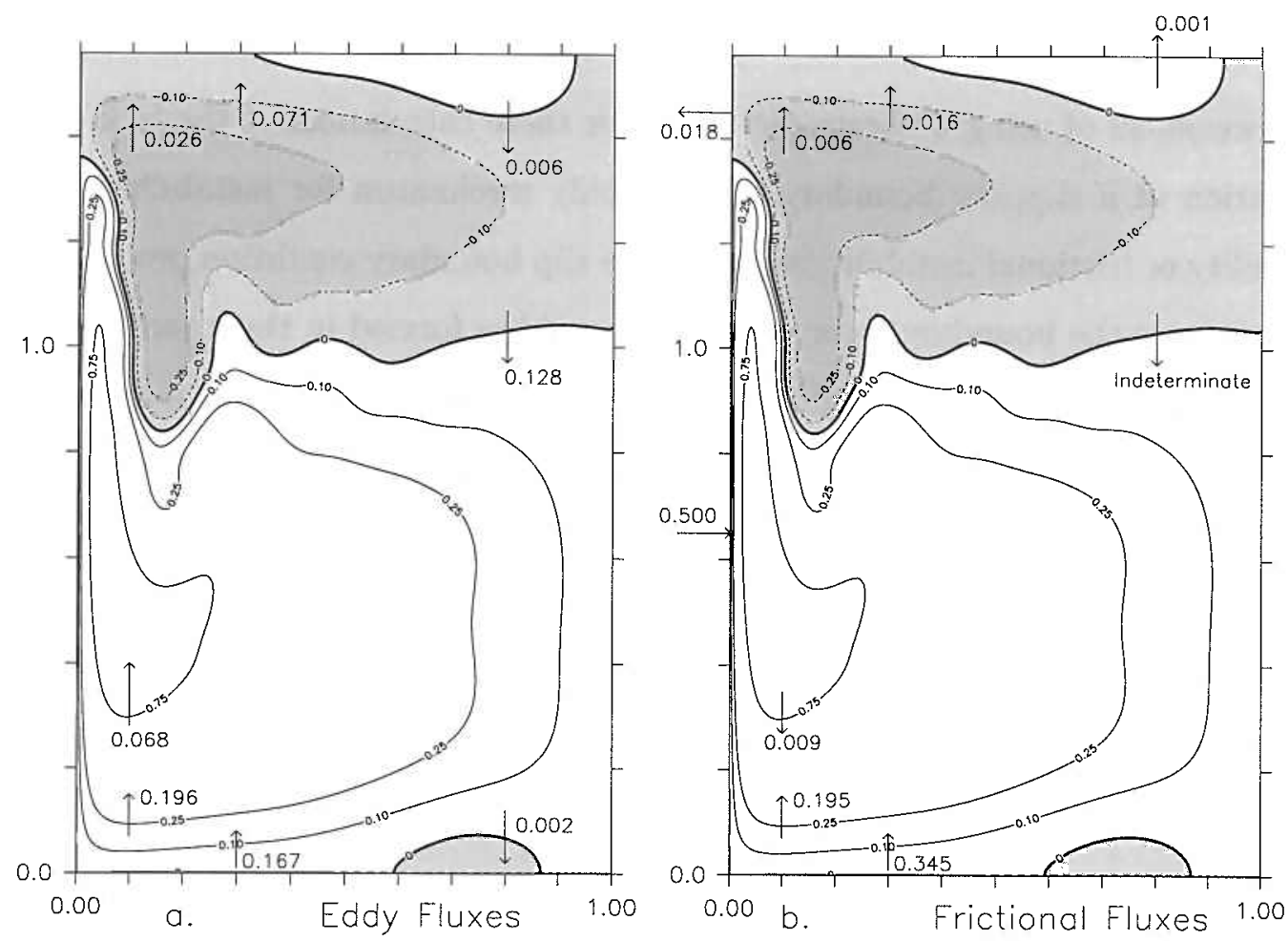

Figure 4-13: As in figure 4-5 except the calculation shown is the slip, two-gyre, $\operatorname{Re}(\mathrm{bdy})=2.5, \operatorname{Re}(\mathrm{int})=2.5$ calculation. Note that only selected streamlines are displayed.

gyre calculation in figure 4-10, the inter-gyre eddy flux is greatly reduced and the eddy flux to the frictional sublayer is increased.

In the double-gyre calculation of figure 4-10, the boundary current from one gyre was forced toward the interior at the center of the western boundary by the opposing boundary current. Because these two currents were equally matched, neither could dominate (at least not for an extended period). In the two-gyre calculation, the currents are not evenly matched.

Cessi (1991) finds that the stronger jet overshoots with slip boundary conditions, and the stronger jet undershoots (separates just before the linear meeting point) with 
no-slip boundary conditions. Her model uses the same dynamical equations as those used here, but the boundary currents' mass transport is from sources on the western boundary to the north and south of the separation to a sink on the eastern boundary. Thus, the issue of the vorticity transport of the boundary current's overshoot (or lack thereof) is not important as it is here. However, the mechanisms described by Cessi (1991) are applicable here, and the overshoot in the slip case is clearly visible in figure 4-13 while a small undershoot occurs in figure 4-7.

In figure 4-13, we see that the inter-gyre eddy flux is smaller (0.128) and the flux to the frictional boundary layer is larger (approximately 0.07) than in figure 4-10. However, we see already that the combined effect of friction (0.500) and inter-gyre eddy flux $(0.128)$ is less than the 0.637 input. The deficit is made up by a mean flux of vorticity in the extended western boundary current of 0.01 units. ${ }^{1}$ The northwardflowing water and southward flowing water have different vorticity because the water experiences the opposing wind forcing after it crosses the zero wind stress curl line. ${ }^{2}$.

The mean flow removing 0.01 units is not much when compared to the 0.64 units of the wind forcing. However, the dominance of the subtropical current becomes even more pronounced for higher Reynolds number calculations. In figure 4-14, the intergyre eddy flux is only 0.10 , so that the mean flow now cancels 0.038 . In figure $4-15$ the boundary current of the subtropical gyre continues northward to form a recirculation gyre which nearly replaces the subpolar gyre. In this case, the inter-gyre eddy flux cancels only 0.038 , so the mean flow cancels 0.10 .

In figure 4-15, the importance of the mean flows across $y=1$ is greater than in figure 4-13. The mean flow of both the subtropical and subpolar gyres carries the water across the zero wind-stress curl line so that the mean flow experiences both wind stresses in turn. The exposure to subtropical wind stress that the subpolar gyre experiences by extending below $y=1$ reduces its vorticity input by 0.01 . The

\footnotetext{
${ }^{1}$ This value is small, but statistically significant.

${ }^{2}$ The extension of the western boundary current also increases the length of the frictional boundary layer, but this effect is included in the 0.500 frictional vorticity flux
} 

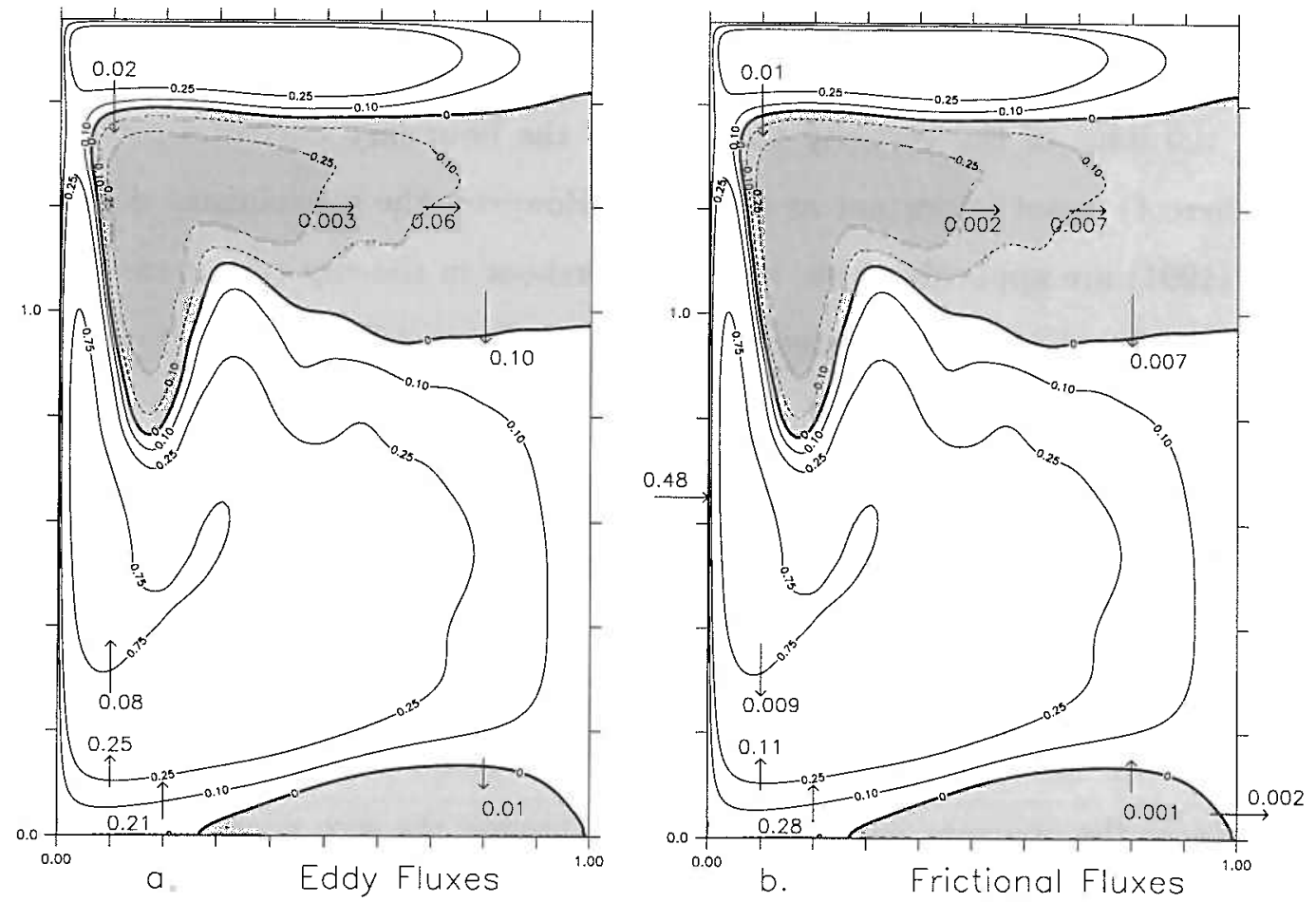

Figure 4-14: As in figure 4-5 except the calculation shown is the slip, two-gyre, $\operatorname{Re}(\mathrm{bdy})=5, \operatorname{Re}(\mathrm{int})=5$ calculation. Note that only selected streamlines are displayed.

exposure to subpolar wind stress that the subtropical gyre experiences by extending above $y=1$ reduces its vorticity input by 0.09 . Therefore, instead of the wind input of -0.637 that the subtropical gyre would ordinarily experience, it receives only -0.521 (not considering the counter-rotating region). Likewise the subpolar gyre experiences 0.054 instead of 0.157 . The effect of the mean flow on the subtropical vorticity input is more than double the 0.05 that is canceled by an internal eddy flux of vorticity. ${ }^{3}$

Although, for what remains of the subpolar gyre, the inter-gyre eddy flux remains a critical outlet. The eddy flux to the extended boundary current to its west removes

\footnotetext{
${ }^{3}$ Because of the high Reynolds number used here, the eddy fluxes are not as accurate as in the other figures, so the number of digits is reduced accordingly.
} 

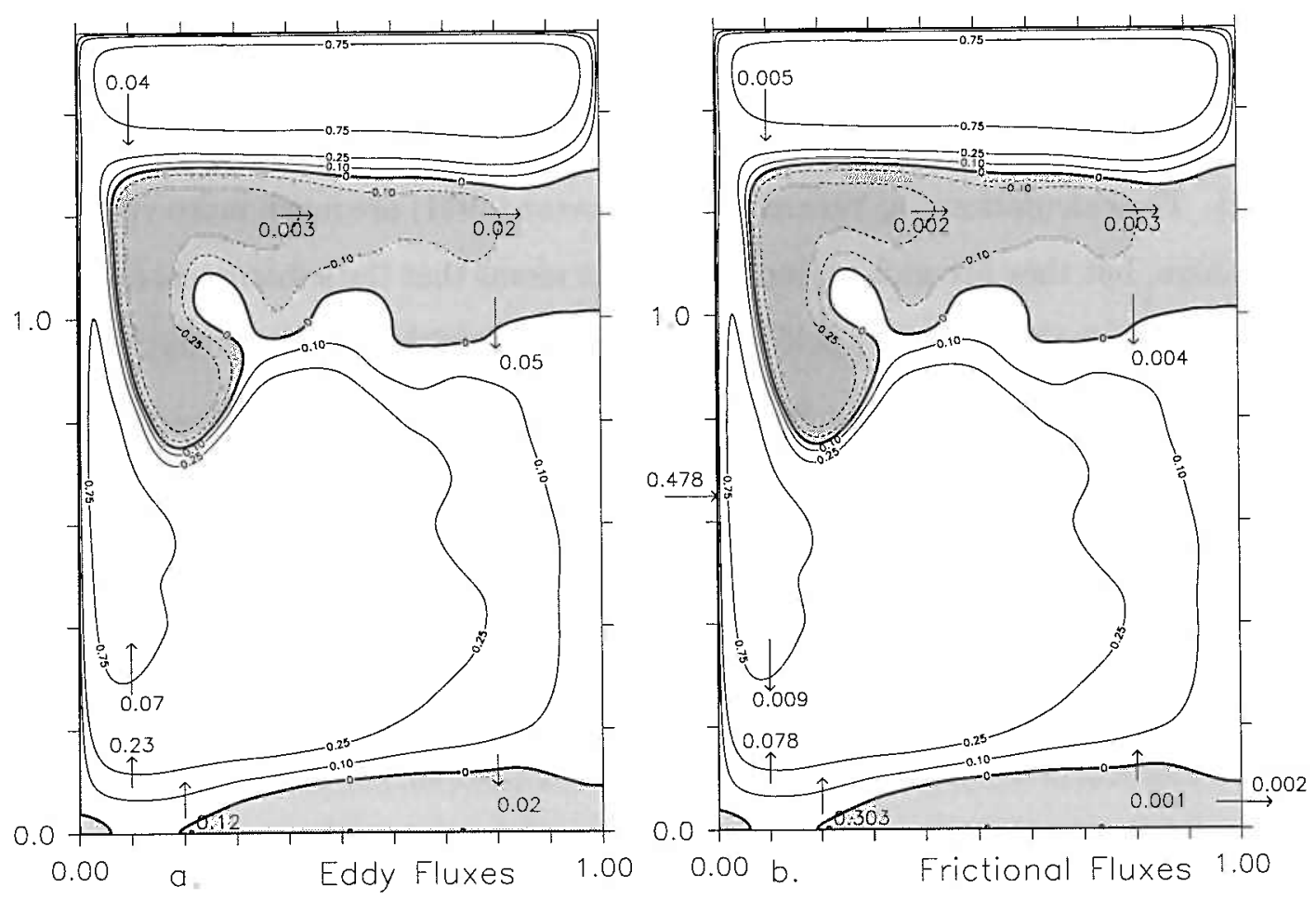

Figure 4-15: As in figure 4-5 except the calculation shown is the slip, two-gyre, $\operatorname{Re}(\mathrm{bdy})=10, \operatorname{Re}(\mathrm{int})=10$ calculation. Note that only selected streamlines are displayed.

nearly all of its vorticity input to the remaining subpolar gyre. This is evidently required, as the subpolar gyre no longer reaches the western boundary for significant dissipation to occur.

Thus, although the mean flux of vorticity only represents a cancellation of approximately 0.01 of the 0.637 total vorticity input in figure $4-13$, this appears to be the preferred method of adjustment in an asymmetric multiple-gyre solution with slip boundary conditions as the Reynolds number increases. Rather than an increase in the inter-gyre eddy flux of vorticity, the subtropical western boundary current intrudes farther and farther into the subpolar gyre. Ultimately, it seems there will be 
only one gyre, which has a recirculation gyre completely filling the region of subpolar wind forcing. In figure 4-15, the inter-gyre eddy flux cancels only a small part of the vorticity input to the subtropical gyre.

A much less dramatic version of this result was seen in Verron and Le Provost (1991). The calculations by Verron and Le Provost (1991) are much more viscous than those here, but they are multi-layered. Thus, it seems that the subtropical domination may also occur at higher Reynolds number even in multi-layered calculations.

Another advantage of extending the western boundary current instead of an intergyre eddy flux is that the boundary current is now much longer. So, instead of removing 0.157 through the boundary between $y=1$ and $y \approx 1.56$ and -0.637 from the $y=0$ to $y=1$ region, it now has to remove only 0.478 and has a longer time to do it (as the current now runs from from $y=0$ to $y \approx 1.56$ and along the northern and eastern boundary as well).

In addition to the mean flow transporting vorticity across the zero wind stress curl line, the mechanism of the preceding chapters is moderately present in the subtropical gyre in figure 4-14 and strongly present in the subtropical gyre in figure 4-15. There is a large eddy flux divergence between the $\psi=0$ and $\psi=0.25$ mean streamlines. This eddy flux divergence is balanced primarily by the frictional flux convergence near the western boundary.

Figure 4-16 demonstrates that effect of the subpolar wind forcing is not negligible in the two-gyre slip calculations. In figure 4-16b, the subpolar wind forcing is set to zero, and the circulation strength is significantly stronger there than in figure 416a. The maximum of the streamfunction in the subpolar region in figure $4-16 \mathrm{~b}$ is approximately 1.98 while it is 0.66 in figure $4-16 \mathrm{a}$. The difference between these two (1.32) is nearly three times the maximum magnitude of the linear Munk solution for the subpolar gyre wind forcing $(-0.48)$. Thus, although the overshoot occurs whether there is subpolar wind forcing or not, the subpolar circulation strength is quite different in the two cases, and the difference is greater than would be expected 

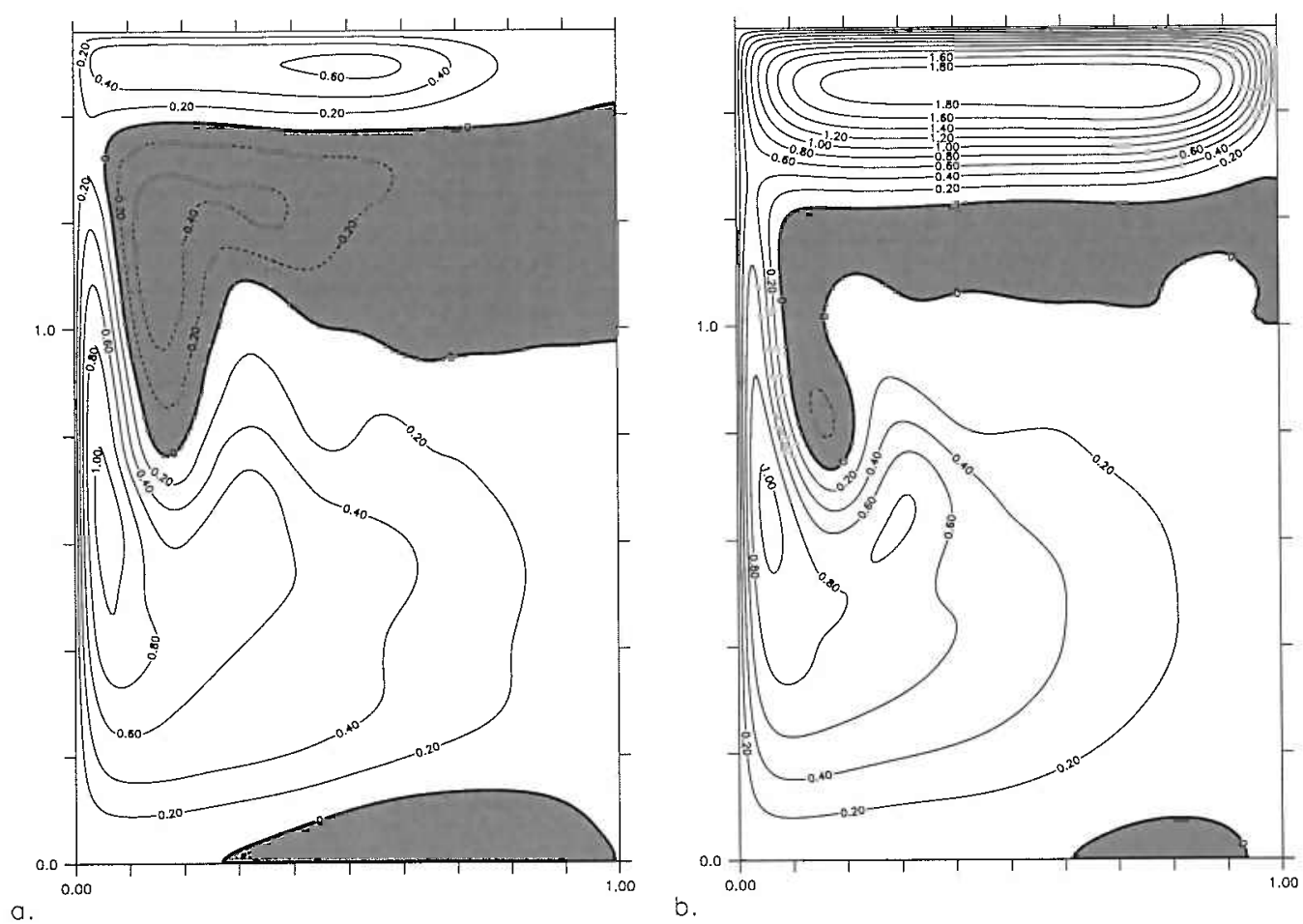

Figure 4-16: Comparison of two-gyre slip calculations with constant viscosity $(\operatorname{Re}(\mathrm{bdy})=\operatorname{Re}(\mathrm{int})=5$ ) with different subpolar wind forcings. (a) uses the standard two-gyre wind forcing, and (b) has wind forcing set to zero in the subpolar gyre.

by considering a linear Sverdrup solution perturbation.

The subtropical gyre, however, is quite similar in figures 4-16a and 4-16b. In both cases, it is nearly in Sverdrup balance; the import of subpolar vorticity into the subtropical gyre does not appear to be a major factor in determining the subtropical circulation strength. Thus, the subpolar wind forcing does affect the strength of the subpolar gyre, but it does not affect the strength of the subtropical gyre.

The export of subtropical vorticity to the region of subpolar wind forcing is important for keeping the subtropical gyre nearly in Sverdrup balance. The exported subtropical vorticity is removed primarily by the elongated western boundary current, 


\begin{tabular}{|c|c|c|c|c|}
\hline \hline Calculation from figure: & $4-13$ & $4-14$ & $4-15$ & $4-16$ \\
\hline Re(bdy)=Re(int) $=$ & 2.5 & 5 & 10 & 5 (no subpolar wind) \\
\hline \hline Wind input $y<1$ & -0.63 & -0.62 & -0.61 & -0.63 \\
\hline Friction $y<1$ & 0.35 & 0.26 & 0.19 & 0.26 \\
\hline Eddy Flux $y<1$ & 0.03 & 0.08 & 0.06 & 0.03 \\
\hline Wind input $y>1$ & 0.01 & 0.05 & 0.09 & 0 \\
\hline Friction $y>1$ & 0.15 & 0.23 & 0.30 & 0.36 \\
\hline Eddy Flux $y>1$ & 0.09 & 0.00 & -0.03 & -0.04 \\
\hline Mean Flux from & -0.25 & -0.28 & -0.36 & -0.33 \\
$y<1$ to $y>1$ & & & & \\
\hline
\end{tabular}

Table 4.1: Regions and mechanisms of vorticity input and removal for the subtropical gyre and its overshoot (i.e., where $\bar{\psi}>0$ ) for the slip, two-gyre calculations. Fluxes are calculated by integration of flux divergence over region where $\bar{\psi}>0$ and $y<1$ or $\bar{\psi}>0$ and $y>1$.

but the subpolar wind forcing also plays an important role. The sources and sinks of vorticity for the region rotating in the anticyclonic direction (i.e., where $\bar{\psi}>0$ ) are given in table 4.1. As the Reynolds number increases the removal of exported vorticity by friction decreases while the effect of subpolar wind forcing increases.

However, because the inner streamlines do not enter the frictional sublayer and the slip boundary conditions are not conducive to a flux to the frictional sublayer, the subpolar wind forcing is important in removing the vorticity from the innermost streamlines as demonstrated by figure 4-16. Table 4.1 indicates that the calculation without subpolar wind forcing has a larger mean inter-gyre flux than the case with subpolar wind forcing at the same Reynolds number. ${ }^{4}$ In the case with subpolar wind forcing, the innermost streamlines have wind forcing to cancel the incoming subtropical vorticity. In the case without subpolar wind forcing, the innermost streamlines trap vorticity so the circulation strengthens until dissipation is able to reach these innermost streamlines.

The dissipative meandering of the double-gyre, slip calculations can be considered

\footnotetext{
${ }^{4}$ This highlights the importance of a regional study with a model such as the one used by Cessi (1991). The global vorticity budget will depend on the local dynamics which determine the strength of the overshoot.
} 
as a special case of the stronger gyre overshooting into the weaker gyre as in the two-gyre calculations. Apparently, whichever of the two gyres in the double-gyre calculation is stronger overshoots into the other one. The resulting elongation of the boundary current allows for increased dissipation (and to a lesser extent exposure to opposing wind forcing), just as in the two-gyre calculations. The difference is that in the double-gyre, once the overshoot has occurred for a while, the stronger gyre weakens because its boundary current is longer. Likewise, the weaker gyre becomes stronger because its boundary current is shorter. Soon, the weaker gyre has become the stronger and the direction of the overshoot reverses. Time-averaging over the alternating overshoots gives a rectified inter-gyre eddy flux, and this is precisely the mechanism of dissipative meandering. No equilibria with the overshoot in one direction or the other were found in the double-gyre, but it is possible that they exist but require a different initial condition to be reached.

Figure 4-17 shows the difference in the frictional flux through the boundary for the two-gyre slip and no-slip calculations. Note that the no-slip calculation has vorticity smoothly injected through the western boundary, so that the vorticity is removed at at almost the same latitude where the wind inputs it. In that case, the frictional removal is quite similar to the linear Munk solution's frictional removal. However, we know that unlike the linear solution, the flux across the interior streamlines is carried by an eddy flux, not a frictional flux.

The two-gyre slip calculation, on the other hand, has a positive vorticity flux all along the western boundary. Thus, the frictional flux is canceling primarily the subtropical wind input, and the subpolar input is canceled internally by the eddy fluxes and the mean fluxes across the zero wind stress curl line. Note that the magnitude of the fluxes through the western boundary is much smaller in the slip calculation than in the no-slip calculation. This is a sign that 1) because the boundary current is elongated, less vorticity per unit length is required to balance the wind input, and 2) the vorticity gradients are weaker at the boundary in the slip calculation than in 


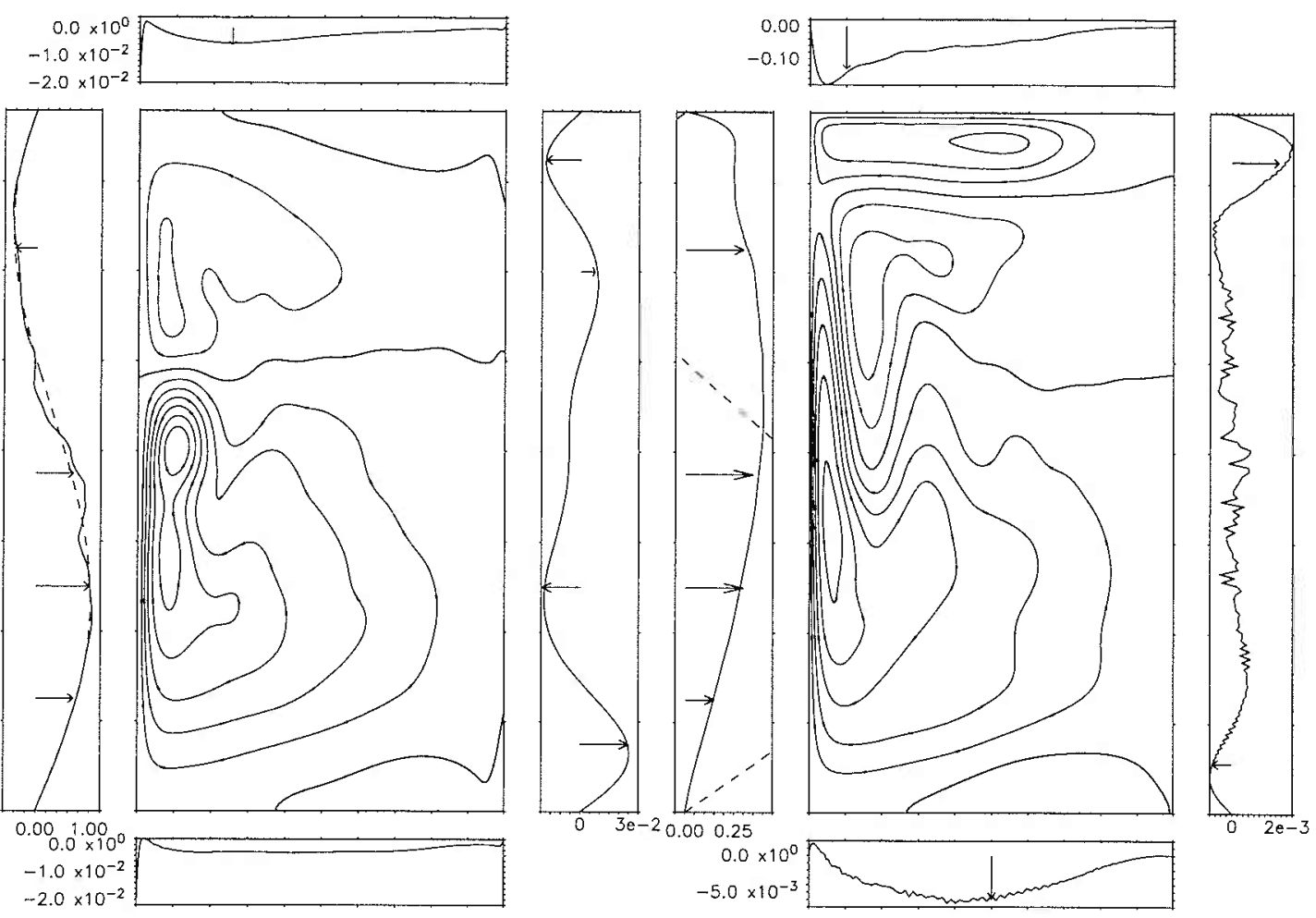

Figure 4-17: Maps of the normal frictional flux through each of the boundaries for the no-slip $\operatorname{Re}(\mathrm{bdy})=5, \operatorname{Re}(\mathrm{int})=5$ two-gyre calculation (on left) and the $\operatorname{Re}(\mathrm{bdy})=5$, $\operatorname{Re}($ int $)=5$ two-gyre slip calculation (on right). The four plots surrounding each contour plot indicate the frictional flux through the nearest boundary to each box $\left(-\delta_{M}^{3} \nabla \zeta\right)$ as a function of distance along the boundary. The flux through the western boundary needed to remove the wind stress input at the same latitude (as in the linear Munk solution) is overlaid with dashed lines. Arrows denote the direction of the frictional flux of positive vorticity. Note that the scales of the flux plots are different.

the no-slip calculation as expected.

Figure 4-18 shows the distribution of absolute vorticity for different two-gyre calculations. Figures 4-18(a-c) demonstrate that as the boundary current overshoots in the two-gyre slip calculations, it advects mean flow relative vorticity. The recirculation that forms at the northern boundary is formed from this advected relative vorticity, and it is relatively homogenized, just as the recirculation gyre is in the single-gyre calculations. The two-gyre no-slip calculation in figure 4-18d also shows evidence of 

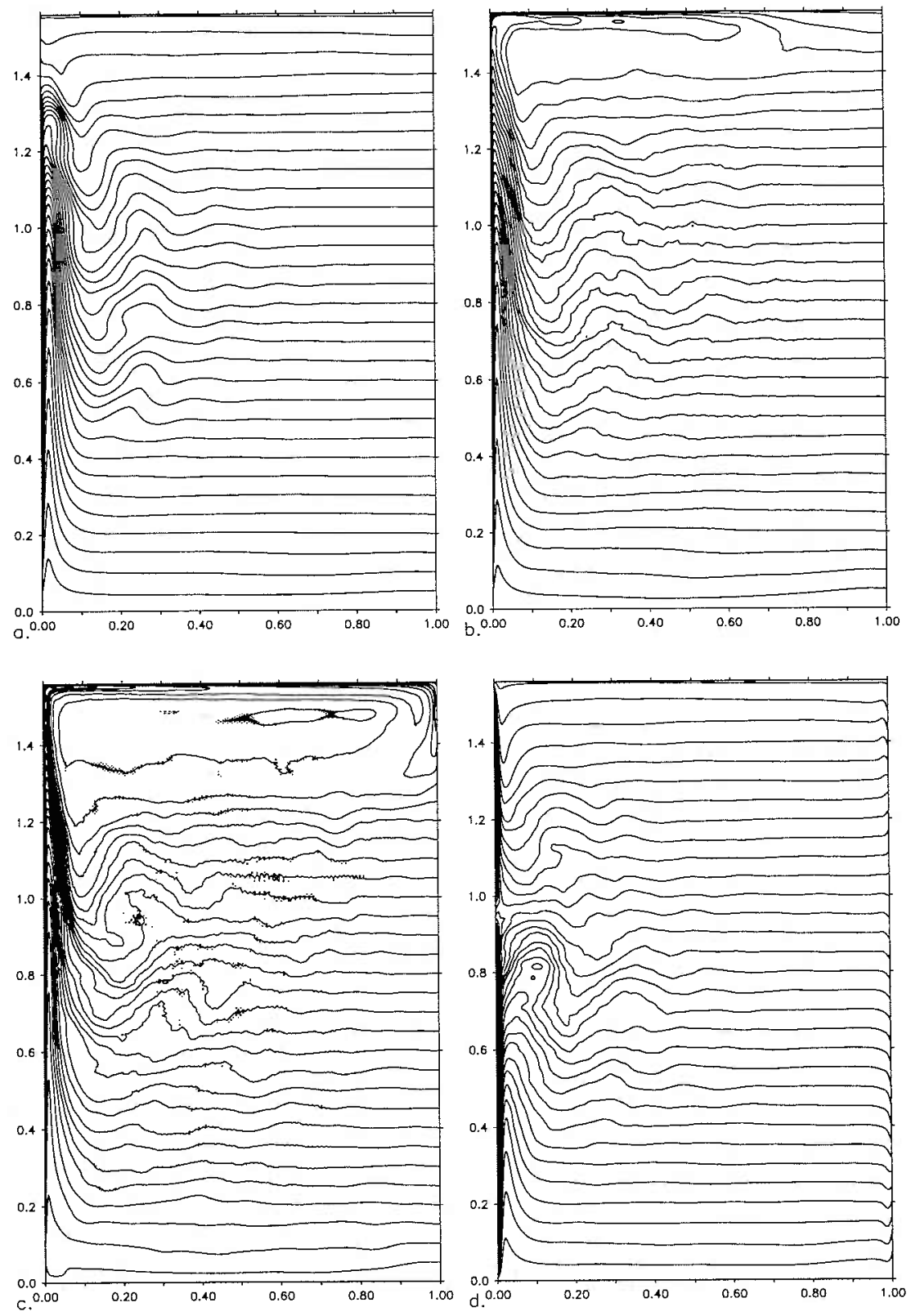

Figure 4-18: Contours of the absolute vorticity for the (a) slip, $\operatorname{Re}(b d y)=2.5$, $\operatorname{Re}($ int $)=2.5$, two-gyre calculation, (b) slip, $\operatorname{Re}(\mathrm{bdy})=5, \operatorname{Re}(\mathrm{int})=5$, two-gyre calculation, (c) slip, $\operatorname{Re}(\mathrm{bdy})=10, \operatorname{Re}($ int $)=10$, two-gyre calculation, and (d) the $\operatorname{Re}(\mathrm{bdy})=5$, $\operatorname{Re}($ int $)=5$ two-gyre no-slip calculation. Contour interval 0.05 . 


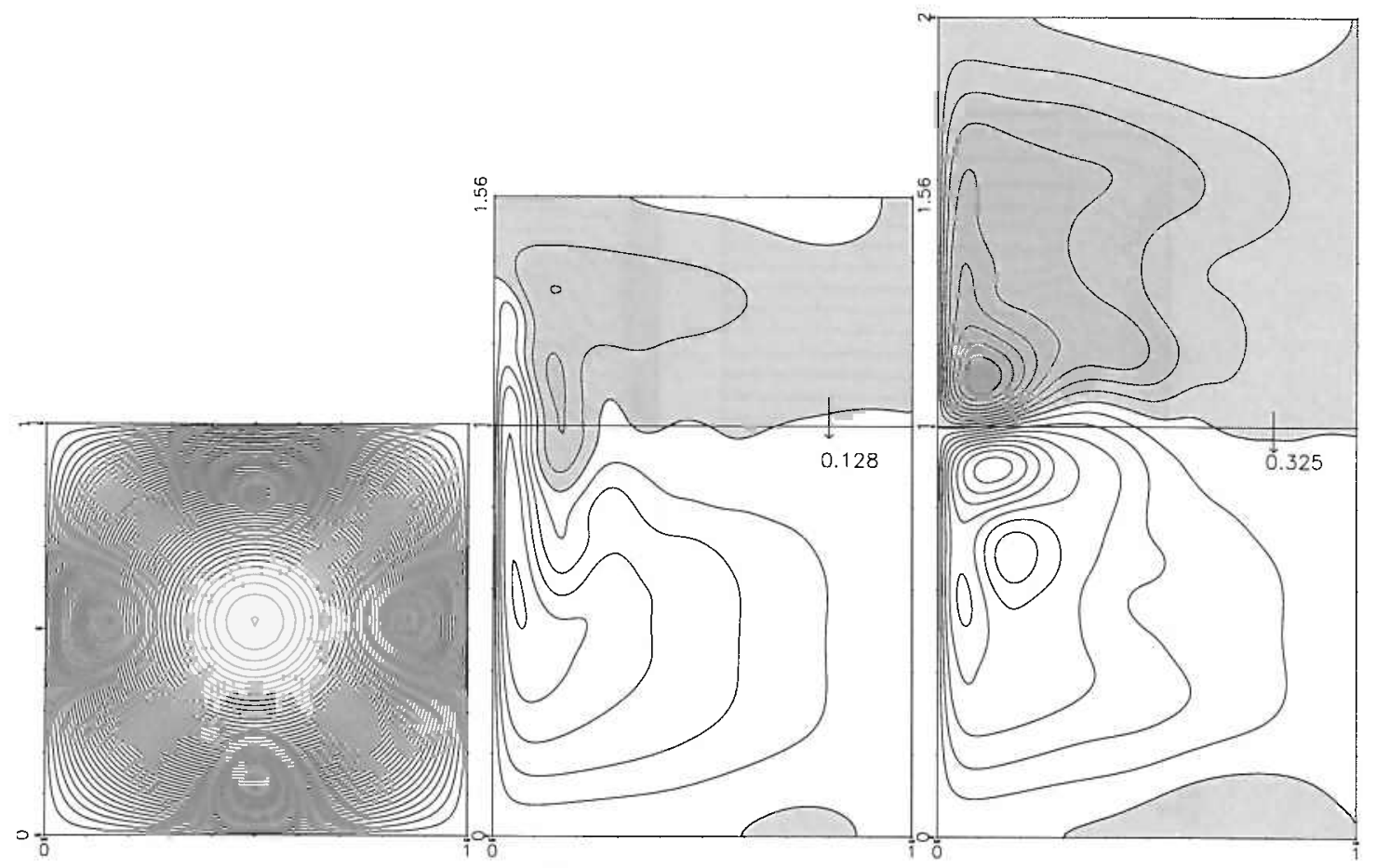

Figure 4-19: Comparison of contours of the time-mean streamfunction for different basins and wind forcing with $\operatorname{Re}(\mathrm{bdy})=2.5, \operatorname{Re}(\mathrm{int})=2.5$, and slip boundary conditions. Regions of negative streamfunction are shaded. The arrows on the figure give the eddy flux of vorticity across that zero contour of psi. (The total vorticity input from the wind to the subtropical gyre is 0.637 ). The contour interval is 0.2 in (b-c) and it is 20 in (a).

advection of relative vorticity, but as the boundary current does not overshoot with no-slip boundary conditions, the advection is toward the basin interior. There is only a small region of pooled vorticity in the no-slip case, as expected because there is not a large recirculation gyre.

Figures 4-19 and 4-20 summarize the results of the single-, two-, and double-gyre calculations with slip boundary conditions. In the single-gyre with slip boundary conditions, a lack of eddy activity requires an enormous circulation strength so that frictional dissipation can balance the wind input. In the two-gyre case, an inter-gyre flux of vorticity by both the mean and eddy fields reduces the amount of vorticity to be removed by friction. By comparing with results at higher Reynolds number, 


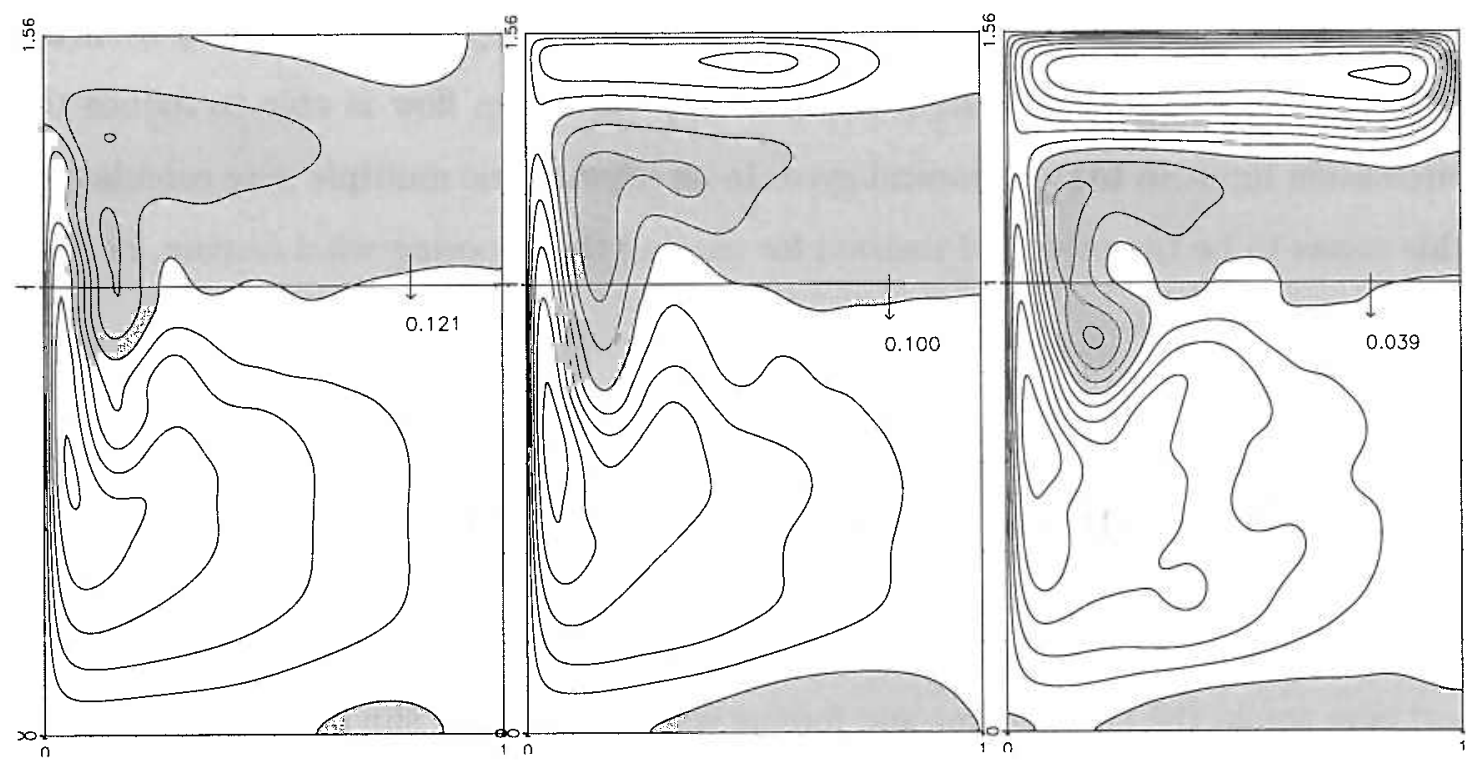

Figure 4-20: Comparison of two-gyre slip calculations with constant viscosity $(\operatorname{Re}(b d y)=\operatorname{Re}($ int $)=\operatorname{Re})$ and increasing Reynolds number. The arrow indicates the eddy flux across the mean streamline dividing the gyres. (a) $\operatorname{Re}=2.5$, (b) $\operatorname{Re}=5$, and (c) $\operatorname{Re}=10$

it seems that the mean flow is the preferred mode of reducing the vorticity input, probably because it lengthens the western boundary current. Some of the eddies generated by instabilities of the jet also allow for an eddy flux of vorticity to the frictional sublayer. Finally, in the singular case of the double-gyre, the antisymmetry of the wind forcing prevents the inter-gyre mean flow, so the eddy flux is the only transport and primary remover of vorticity.

In summary, when slip boundary conditions are used for multiple-gyre calculations, the results are quite different from those with no-slip boundary conditions. In the double-gyre, the inter-gyre eddy flux becomes quite large, and is the dominant sink of vorticity input from the wind. Because the slip boundary conditions tend not to create eddies near the boundary as in the no-slip conditions do, the mechanism of an eddy flux to a frictional sublayer is not as important as the inter-gyre flux in the double-gyre. However, when the exact antisymmetry of the double-gyre is broken in the two-gyre calculations, the western boundary current of the subtropical gyre is 
unopposed and overshoots, eventually dominating the subpolar gyre. By experiencing the opposing wind forcing, the fluid carried by this mean flow is able to reduce the circulation input to the subtropical gyre. In an asymmetric multiple gyre calculation, this seems to be the preferred method for sharing the opposing wind forcing, not the inter-gyre eddy flux.

\subsection{Mechanisms for Control of Circulation Strength}

Figures 4-2, 4-4 and 4-19 clearly demonstrate that there is a difference in the subtropical gyre across the three basins and forcing schemes whether slip or no-slip boundary conditions are used. The subtropical gyre circulation strength is much stronger in the single-gyre calculations where a pronounced recirculation gyre forms. In the two-gyre calculations and the double-gyre calculations, the recirculation is significantly weaker, and the circulation is close to the transport in the linear Munk model in the no-slip cases.

The preceding section shows that for slip boundary conditions, an inter-gyre flux of vorticity cancels much of the wind-stress input in the double-gyre calculations. The mechanism of this exchange is primarily the dissipative meandering mechanism proposed by Lozier and Riser (1990), not an exchange of mass. In this section, the reasons for the small amount of mass exchange are explored. Also, the reason why dissipative meandering is active in the slip calculations but not in the no-slip calculations is shown to be that the separation point does not vary in the no-slip calculations. However, as shown in section 4.3 , the dissipative meandering mechanism is not dominant in the asymmetric two-gyre calculations where the mean subtropical flow dominates the subpolar gyre.

The two-gyre and the double-gyre calculations with no-slip boundary conditions have very similar shape and circulation strength (very nearly the Sverdrup transport). Apparently, the mechanism which controls the circulation is relatively insensitive to 
the strength of the subpolar gyre. Also, as was shown in section 4.2 , the inter-gyre eddy fluxes are negligible while the eddy fluxes to a frictional sublayer are strong, just as in the single-gyre calculations. In this section, it will be shown that these results indicate that new modes of instability along the jet are responsible for an increase in the efficiency of the eddy flux to the frictional sublayer, and this increased efficiency prevents the buildup of vorticity which causes the recirculation gyres and reduces the circulation strength.

\subsubsection{Inter-gyre Eddy Transport}

The first hypothetical mechanism for the control of the circulation strength by eddies is that they might exchange mass and vorticity across the inter-gyre boundary by semi-permanent exchange of fluid parcels.

However, it has recently been well-established in the literature that there is little mass transport between the gyres in a wind-driven model, even when there is vorticity transport. Previous numerical simulations have shown that it is difficult to transport mass across the jet (Berloff et al. (2002), and Berloff and McWilliams (2002), Lozier and Riser (1989), and Lozier and Riser (1990)). In fact, Lozier and Riser (1989) found so little mass transport across the boundary current that they proposed the mechanism of dissipative meandering to explain how the vorticity could be transported without mass exchange. Observations of the ocean by Bower and Rossby (1989) also indicate a small mass exchange across the Gulf Stream.

In the laboratory and atmosphere-especially the stratosphere-so-called potential vorticity barriers have been found (see, for example, Haynes and Shuckburgh (2001) and Pierrehumbert (1991)). These regions possess large gradients of potential vorticity, and are barriers to isentropic eddy transport. As pointed out by Pierrehumbert (1991), it is unclear whether the large gradients of potential vorticity are a result of weaker turbulent mixing or causing weaker turbulent mixing.

To address the question of mass transport across and near jets, there have been 
studies of chaotic advection near jets. Pratt et al. (1995) and Rogerson et al. (1999) have analyzed the Lagrangian stirring for idealized quasi-geostrophic and barotropic jet models. In their analysis most of the Lagrangian transport across mean streamlines occurred near pronounced eddies with Kelvin's cat's eyes which form to either side of the meandering jet. Balmforth and Piccolo (2001) find similar cat's eyes structures in solutions of the evolution of a Bickley jet ${ }^{5}$ with dynamical equations identical to those used in this dissertation. Figure 4-21 is a reproduction of a figure from Rogerson et al. (1999) which shows extensive stirring in the location of the cat's eyes, but very little mixing across the jet. Rogerson et al. (1999) proceed with a lobe dynamics analysis of the meandering of this jet. They show that the cat's eyes possess hyperbolic points of the flow in their reference frame. That is, points where mean streamlines converge along one direction and diverge along another. When these hyperbolic points are subject to time-dependent perturbations, as is the case in periodically-varying or dynamically-varying flows, substantial fluxes of mass and conserved properties cross the mean streamlines which approach the hyperbolic points. By numerically locating these points and the trajectories which approach them, Rogerson et al. (1999) are able to demonstrate significant cross-streamline mixing to either side of the jet.

However, they find no transport across the jet. This is because for the eddies to form hyperbolic points within the jet, they would need to be extremely strong; strong enough to halt the jet altogether. In regions of large potential vorticity gradients, the streamlines are bunched very closely together, and so it becomes very difficult to form hyperbolic points within the closely bunched streamlines thereby blocking the transport across them.

Despite differences in the models and forcing, the double-gyre and two-gyre no-slip calculations presented here have regions of large absolute vorticity gradients where the eddy fluxes across mean streamlines are decreased. Figures 4-22 and 4-23 show the regions of large absolute vorticity gradients in the no-slip calculations. The black

\footnotetext{
${ }^{5}$ The Bickley jet is a jet in an infinite plane with initial velocity $U \propto \operatorname{sech}^{2}(z)$ where $z$ is the cross-jet coordinate.
} 
(a)
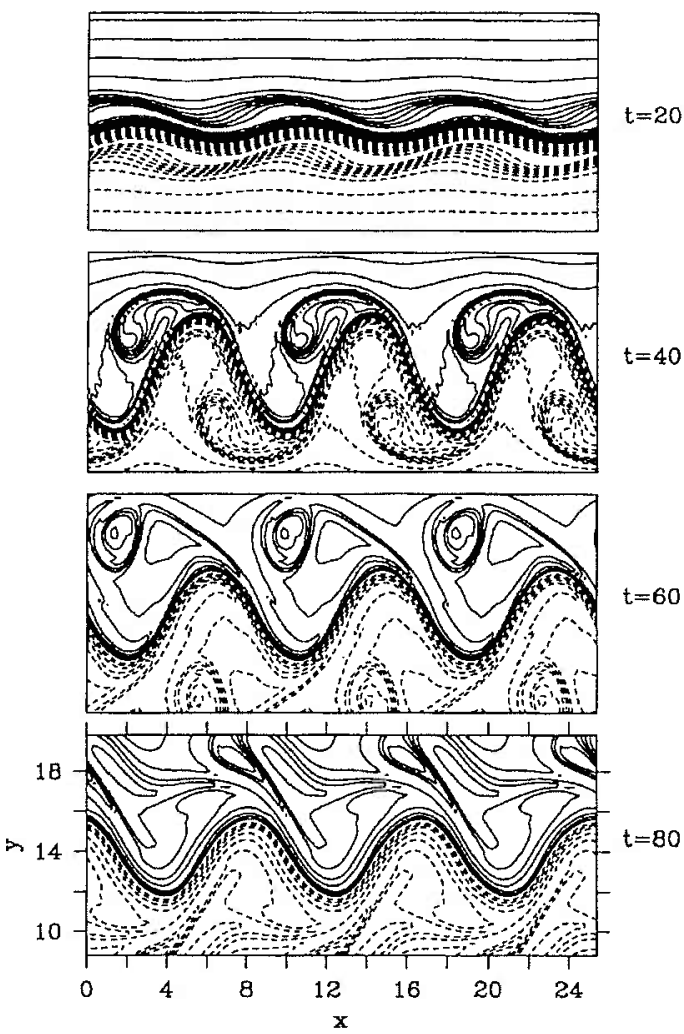

(b)
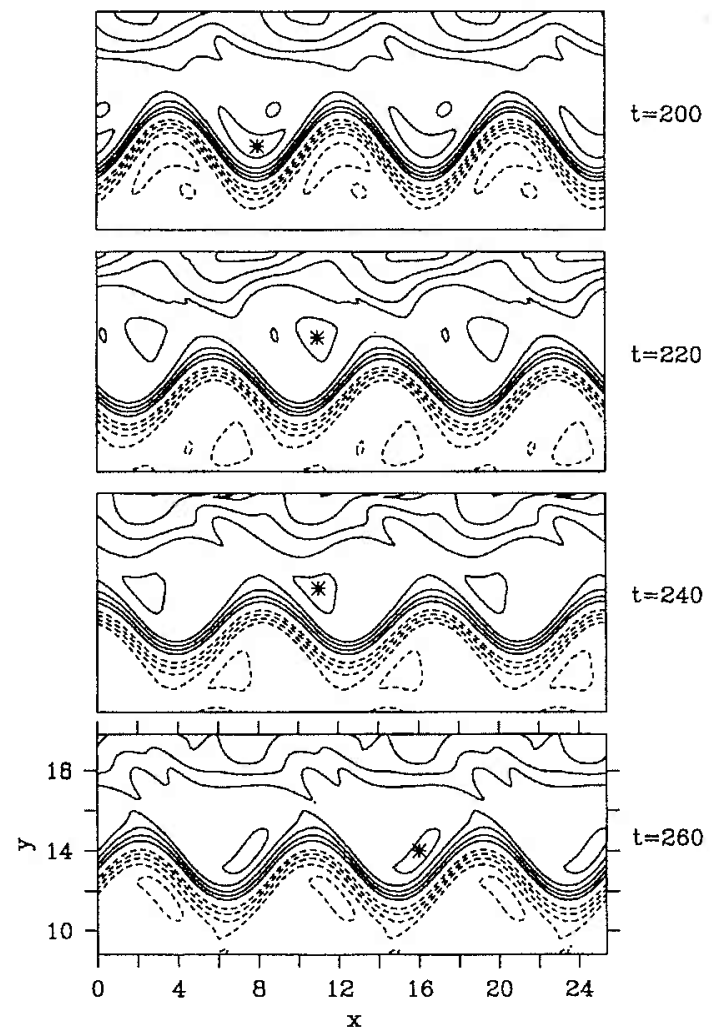

Figure 4-21: Evolution of the flow as represented by the potential vorticity field of a weakly-perturbed meandering barotropic jet (initial streamfunction: $\psi(x, y, t=$ $\left.0)=-\operatorname{erf}(y)+2 y / L_{D}+\epsilon e^{-y^{2}} \sin (k x)\right)$ in a doubly-periodic domain. This figure is reproduced from figure 4 of Rogerson et al. (1999).

regions in these figures show where the gradients of absolute vorticity are large. According to the vorticity gradient barrier theories, these regions are barriers to the eddies. The frictional boundary layer has the largest vorticity gradients, but the gradients extend to the region near the inter-gyre boundary in the calculations with two gyres, presumably carried out of the boundary layer by advection. These regions apparently block eddy transport, both of mass and vorticity in the no-slip calculations. Thus, it seems that it will be rather difficult for eddies to move directly from one gyre to the other, as these vorticity gradients will tend to block their progress.

Figure 4-24 shows that a similar region exists where the jet separates the gyres in 

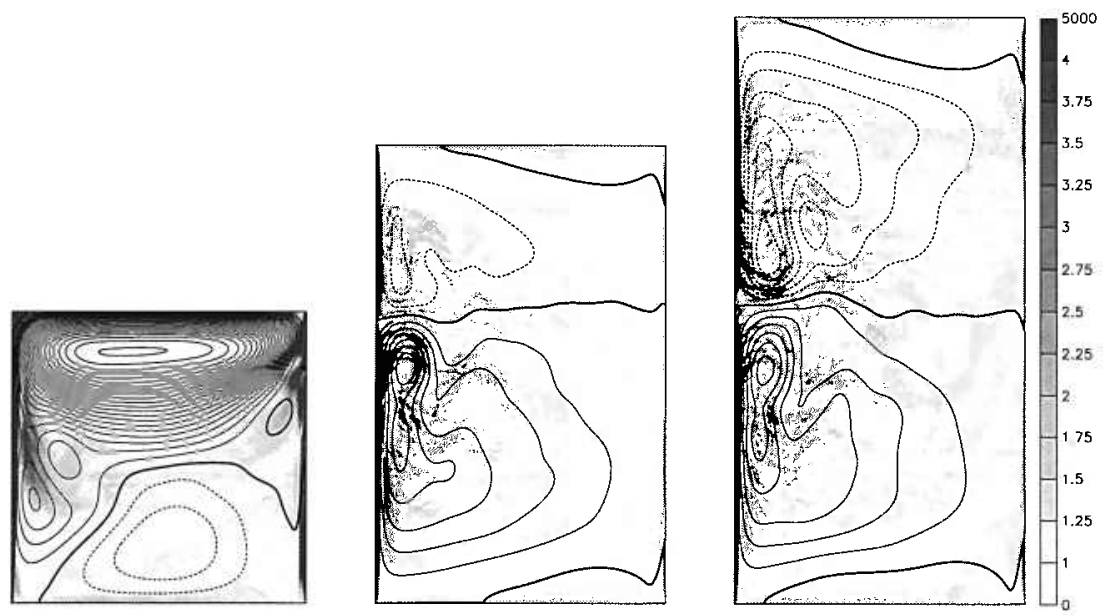

Figure 4-22: Comparison of magnitude of time-mean absolute vorticity gradient squared for different basins and wind forcing with $\operatorname{Re}(\mathrm{bdy})=5, \operatorname{Re}(\mathrm{int})=5$ and noslip boundary conditions. Superimposed is the time-mean streamfunction with a contour interval of 0.2 .
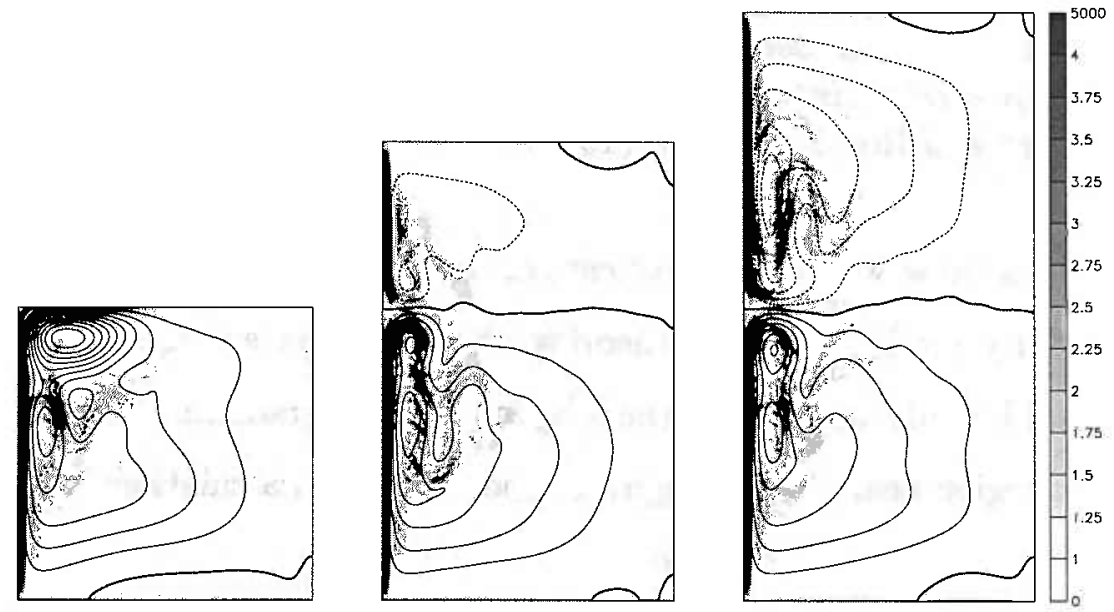

Figure 4-23: As in figure 4-22, except for the $\operatorname{Re}(\mathrm{bdy})=0.25, \operatorname{Re}($ int $)=5$ calculations with no-slip boundary conditions. 

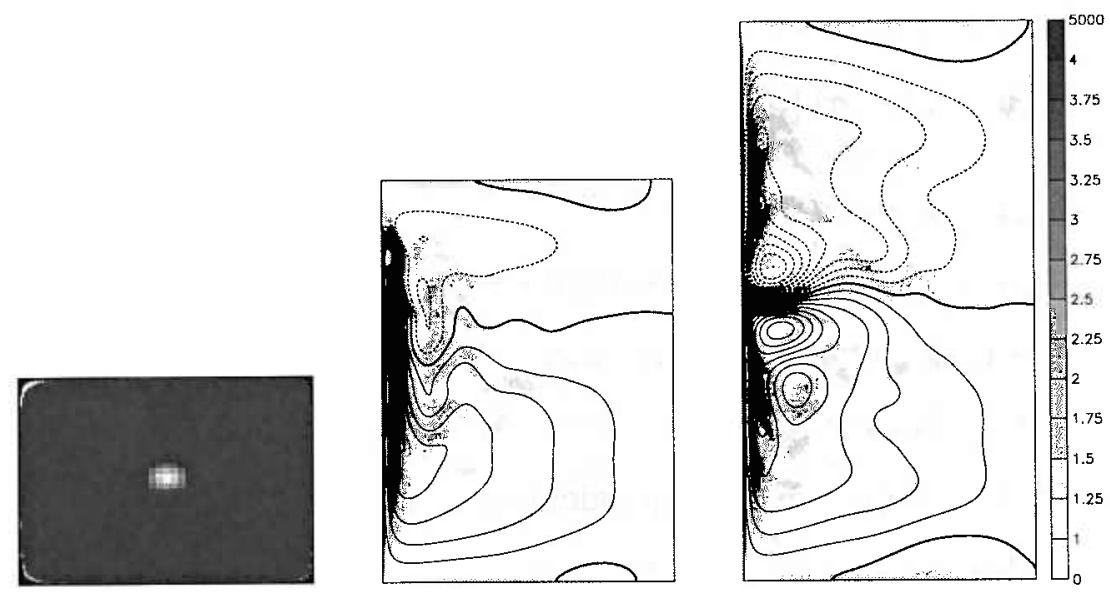

Figure 4-24: As in figure 4-22, except for the $\operatorname{Re}(\mathrm{bdy})=2.5, \operatorname{Re}($ int $)=2.5$ slip boundary condition calculations.

the double-gyre, slip calculation. Again, as demonstrated by previous work, there is little mass transport even with slip boundary conditions, however there is significant vorticity transport across the dividing jet. As pointed out by Lozier and Riser (1990), there must be another mechanism allowing the transport of vorticity across the jet which does not rely on mass transport. This mechanism appears to operate with slip boundary conditions, but not with no-slip boundary conditions.

In summary, it is well established in the literature that little or no inter-gyre exchange of mass occurs with slip boundary conditions for a jet of the strength in these models, and as there is no vorticity transport very little is exchanged with noslip boundary conditions as well. Only irreversible mixing across the center of the jet dividing the gyres could contribute to the inter-gyre vorticity flux. As the pronounced regions of stirring in models similar to this one are located to either side of the jet, with hyperbolic points that are not located in the center of the jet, the stirring does not tend to mix across the center of the jet. Instead, the properties are strongly mixed to either side of the jet, a fact which will be very important to the hypothesis 
to be presented to explain the differences in circulation when a second gyre is added.

\subsubsection{Dissipative Meandering}

Unlike the idea of direct fluid parcel exchange, dissipative meandering does not require irreversible stirring across the jet. Its irreversibility stems from the action of friction. However, there can be little or no dissipative meandering in the no-slip calculations here as there is almost no inter-gyre vorticity flux. At the same time, there is a large amount of dissipative meandering in the slip calculations here and the calculations of Lozier and Riser (1990) (and probably of Marshall (1984) and Harrison and Holland (1981)). As we have seen in the preceding sections, the only significant inter-gyre eddy fluxes here occur in the double-gyre, slip calculations. In this section, we will examine where the eddy flux occurs, how it occurs, and why it does not occur with no-slip boundary conditions.

Consider a vorticity anomaly caused by the displacement of a parcel of subtropical water across $y=1$ in a calculation with slip boundary conditions. With slip boundary conditions, the vorticity of the boundary current is predominantly of the same sign as that of the wind input within that basin. Thus, the displaced parcel of subtropical water is likely to be a negative vorticity anomaly.

If the parcel is in the interior, is it likely to return to its initial position unchanged, and the vorticity anomaly will propagate away reversibly as a Rossby wave, experiencing little dissipation. The isoline of vorticity which is displaced is likely to be left unbroken. As the Rossby wave forms, there will be a negative eddy vorticity flux (negative vorticity traveling north) as the parcel is displaced. However, as the Rossby wave propagates away, a positive eddy vorticity flux (negative vorticity traveling south) will result. As the isoline returns to its initial position, no net eddy flux is achieved.

However, if the parcel is near the boundary, then quite a different process is possible. Now, the isoline of vorticity is displaced northward (a negative eddy flux) 
then some of the anomalous vorticity of the parcel is fluxed by friction out through the western boundary. If the parcel now returns to its original position at the same speed as it originally crossed the inter-gyre boundary, its eddy flux is reduced upon return. Thus the outgoing eddy flux is larger than the returning eddy flux.

Figure 4-25 shows snapshots of a meander near the boundary crossing the intergyre boundary, reducing in strength, and then returning. First, beginning at time 5030 subpolar water is displaced to the south. A large southward eddy flux results until time 5060, when the motion is slowed. Then, the action of dissipation takes over, and the vorticity anomaly returns to the inter-gyre boundary without an intergyre eddy flux. Next, a substantial eddy flux close to the boundary is formed as a subtropical anomaly moves north from time 5070 to 5100 . Then, before the dissipative action has much time to return the vorticity to its average position, another anomaly of relative vorticity north of the inter-gyre boundary appears. As this the positive vorticity anomaly moves south across the inter-gyre boundary in times 5110 to 5115 , again a southward vorticity flux results. The last image shows the average eddy flux divergence, which confirms that, on average, the eddy flux described above results in a noticeable transport of vorticity which starts and ends in the regions where these eddy fluxes tend to start and end during the dissipative meandering.

In the case of no-slip boundary conditions, the vorticity at the boundary current separation point does not move north and south, because the frictional boundary condition demands that the along-boundary velocity that would advect the vorticity must go to zero. Although the fluid further away from the boundary is free to migrate north and south, it will not have a direct connection to the boundary, and therefore the frictional flux acting on it will be decreased. In fact, the condition of what makes the fluid cross the inter-gyre boundary rapidly is precisely how far out of the frictional sublayer it lies. Therefore, the mechanism of dissipative meandering is strongly curtailed and the eddy fluxes are quite different.

Also, unlike the slip boundary current, the no-slip boundary current must possess 

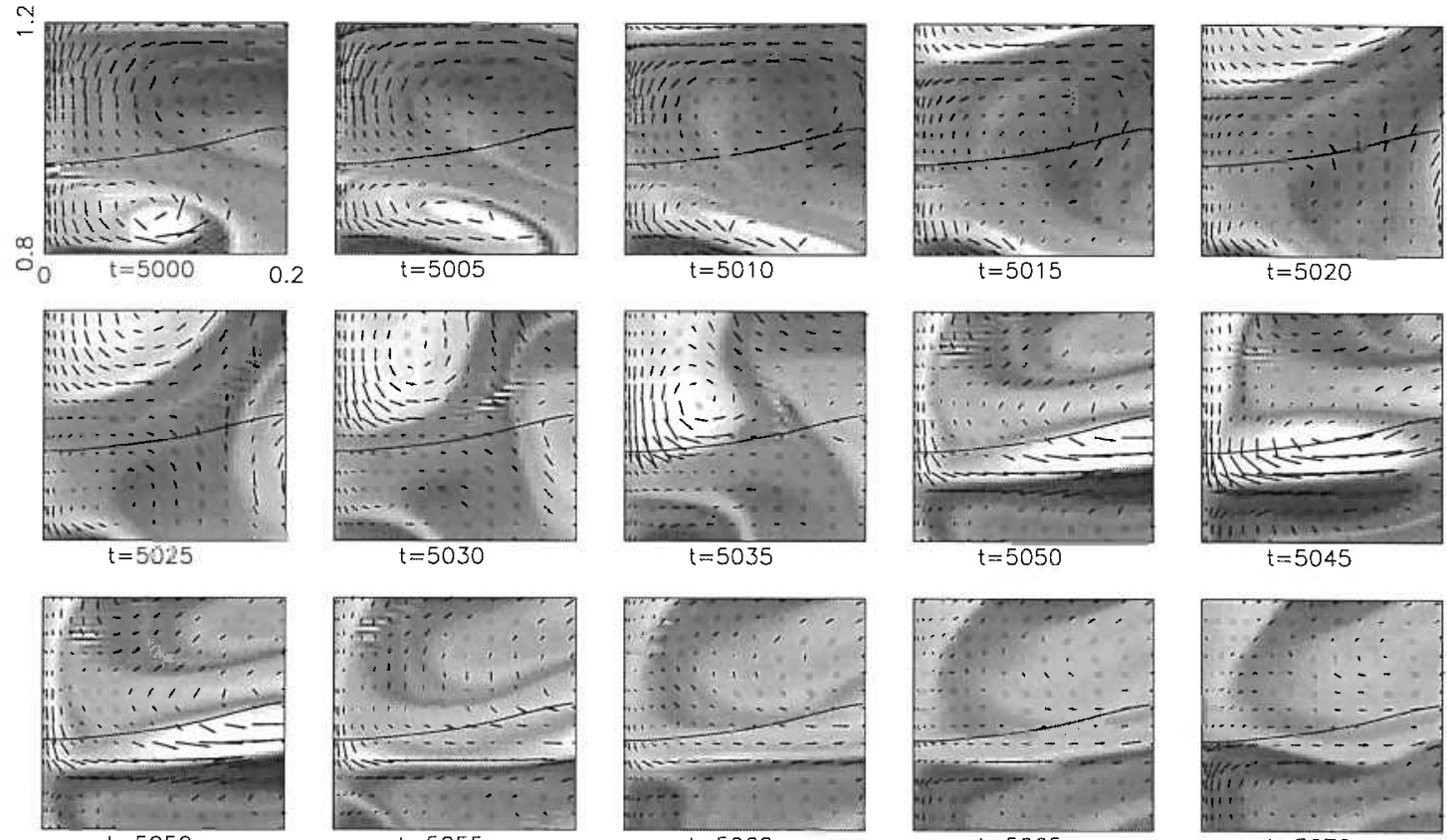

$t=5060$

$t=5050$

$t=5045$
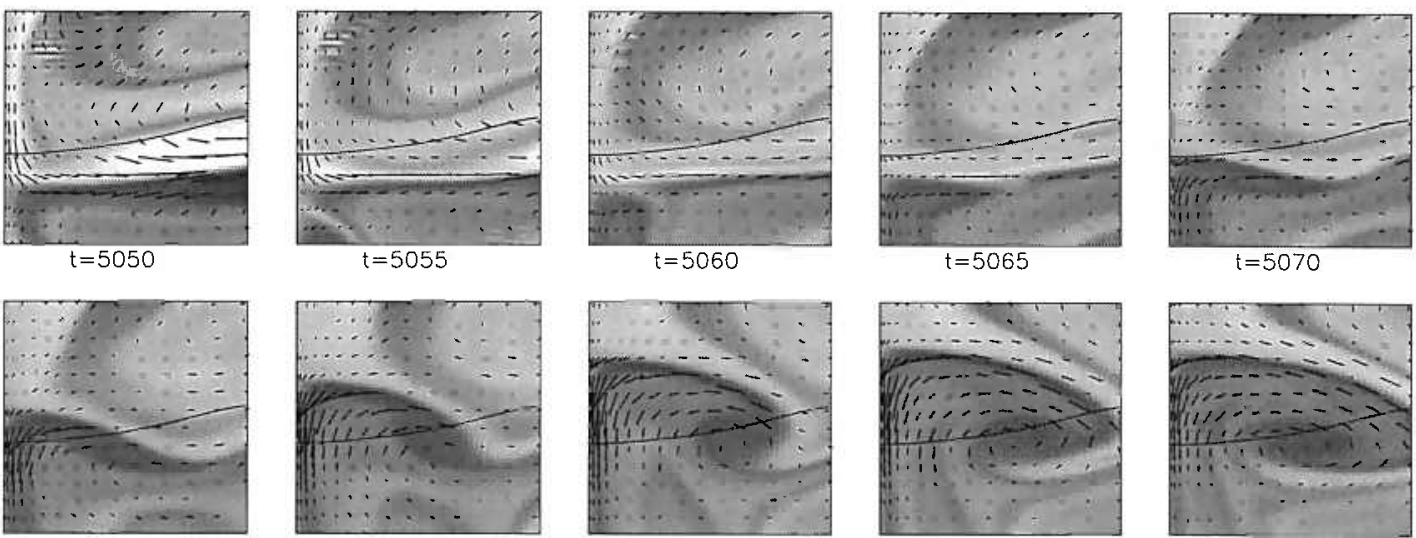

$t=5075$
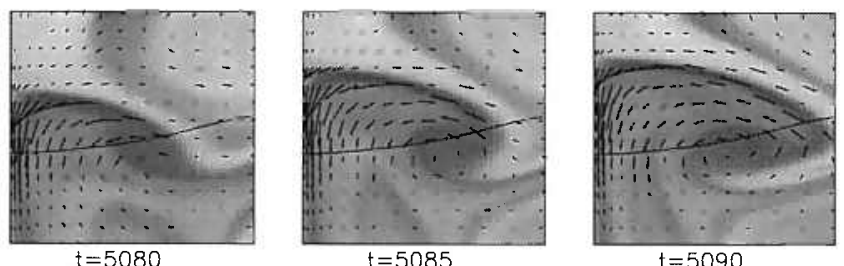

$\mathrm{t}=5090$
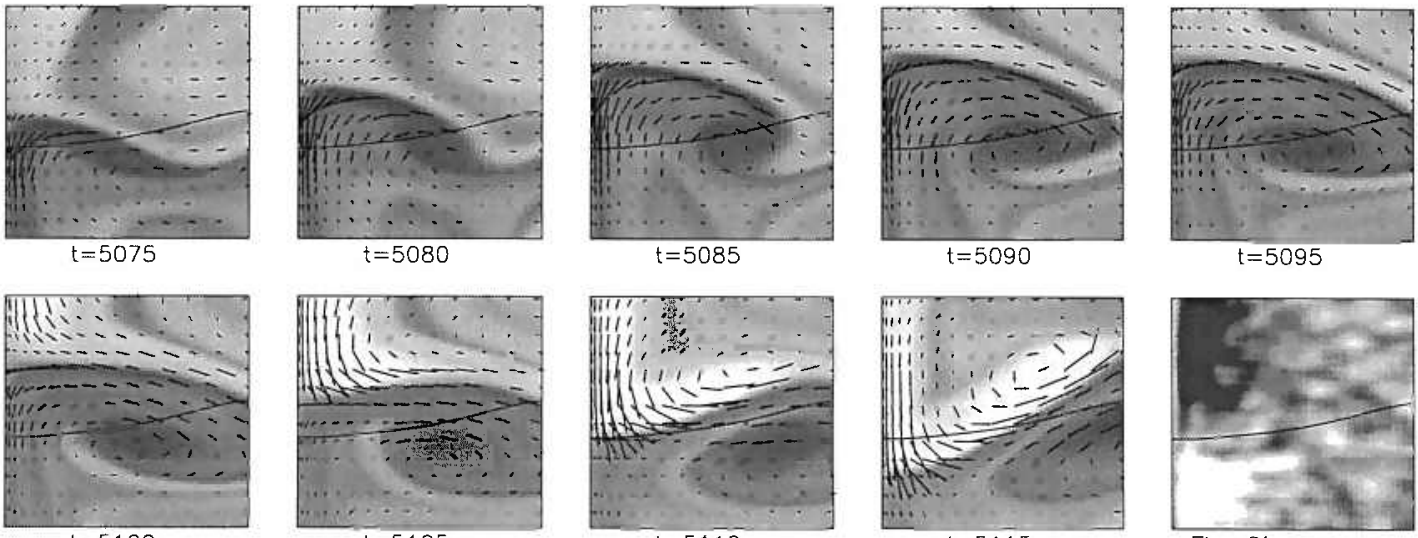

$t=5095$

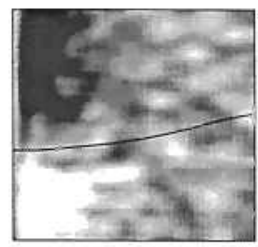

Flux Divergence

Figure 4-25: Snapshots zooming in on the separation region of the slip $\operatorname{Re}($ int $)=\operatorname{Re}($ bdy $)=5$ calculation. Shading denotes relative vorticity, darker is more negative, lighter is more positive. The solid line denotes the average position of the inter-gyre boundary. Arrows denote the local instantaneous value of eddy vorticity flux: $\mathbf{u}^{\prime} q^{\prime}$. Lower right image shows the time-mean eddy flux divergence (dark/light values are divergences/convergences). 
Time-Mean Meridional Velocity ( $v$ )
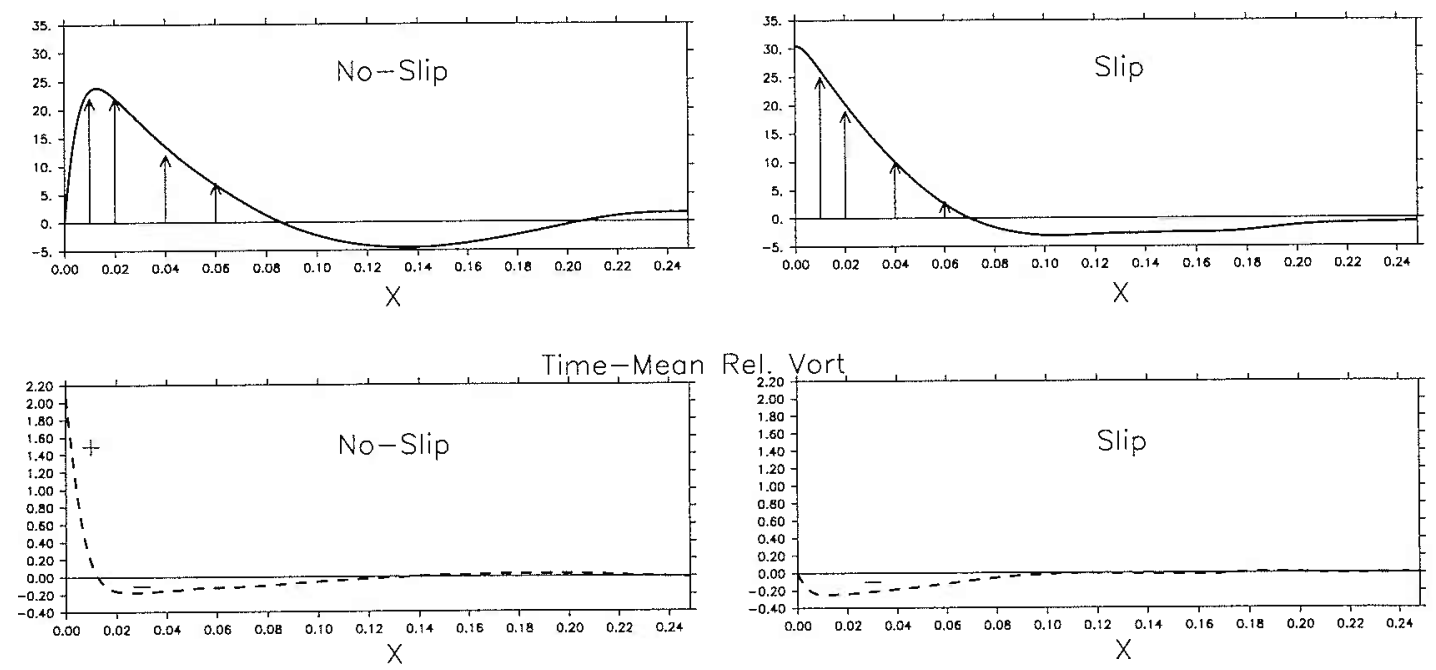

Figure 4-26: Relative vorticity and meridional velocity near the boundary layer at a particular value of $y$ in the subtropical gyre for a slip and a no-slip calculation.

relative vorticity of both signs (see figure 4-26). As one moves away from the boundary, the velocity first increases from zero and then decreases to its interior value. This implies that the relative vorticity is of opposite sign to the wind input of vorticity on the shoreward side of the boundary current and of the same sign as the wind's vorticity input on the seaward side of the boundary current.

Figure 4-27 shows a typical evolution of fluid near the boundary with no-slip boundary conditions. Note that the eddy fluxes across the inter-gyre boundary are quite small. There are significant eddy fluxes, but they tend to be between the boundary and the interior, associated with dipole vortices, a vortex of one sign paired with another of the opposite sign. This dipole structure is directly related to the fact that the boundary current must have relative vorticity of both signs when noslip boundary conditions are used. It is possible for these dipole vortices to cross the inter-gyre boundary, but their vorticity anomalies are cancellatory. The average eddy flux divergence pattern shown in the last image is quite different from that in figure 4-25, as it indicates a strong interaction between the frictional layer near the 
boundary and the interior.

Thus, the inter-gyre flux in the no-slip case is limited to processes which occur some distance away from the boundary, decreasing the effectiveness of dissipative meandering. As mentioned above, the weakly frictional propagation of Rossby waves can produce a small inter-gyre transport. Also, the small amount of mass transport can produce inter-gyre vorticity fluxes. However, as the eddies in the no-slip case tend to be dipole vortices formed in the boundary current, which have cancellatory vorticity fluxes if displaced across the inter-gyre boundary. Other mechanisms will be proposed in the following sections which could account for small amounts of vorticity flux.

Figure 4-28 shows the eddy fluxes across $y=1$ for the calculations shown in figures 4-25 and 4-27. Obviously, the eddy flux is significantly larger in the case of slip boundary conditions. The majority of the eddy fluxes occur in a region near the boundary, which is consistent with the mechanism of dissipative meandering.

Farther away from the boundary, there are smaller vorticity fluxes of alternating sign in the slip fluxes. These fluxes are likely to be due to either the dipole vortex displacements or a correlation in the phase of Rossby waves over the averaging interval, as these vorticity fluxes integrate nearly to zero.

In the small vorticity flux from the no-slip calculation, it is quite convincing that there is little dissipative meandering in the no-slip calculation. In fact, the flux closest to the boundary is of the wrong sign because the relative vorticity within the frictional sublayer is of the opposite sign from the wind forcing with no-slip boundaries. Thus, there are local regions of average vorticity flux even in the no-slip case. However, as these regions integrate almost exactly to zero, they are likely to be due to one of the reversible or cancellatory processes just described.

Thus, the mechanism of dissipative meandering appears to rely critically on the location of the point of jet separation from the boundary and the overwhelming bias of the vorticity anomaly when slip boundaries are used. If this point of separation 

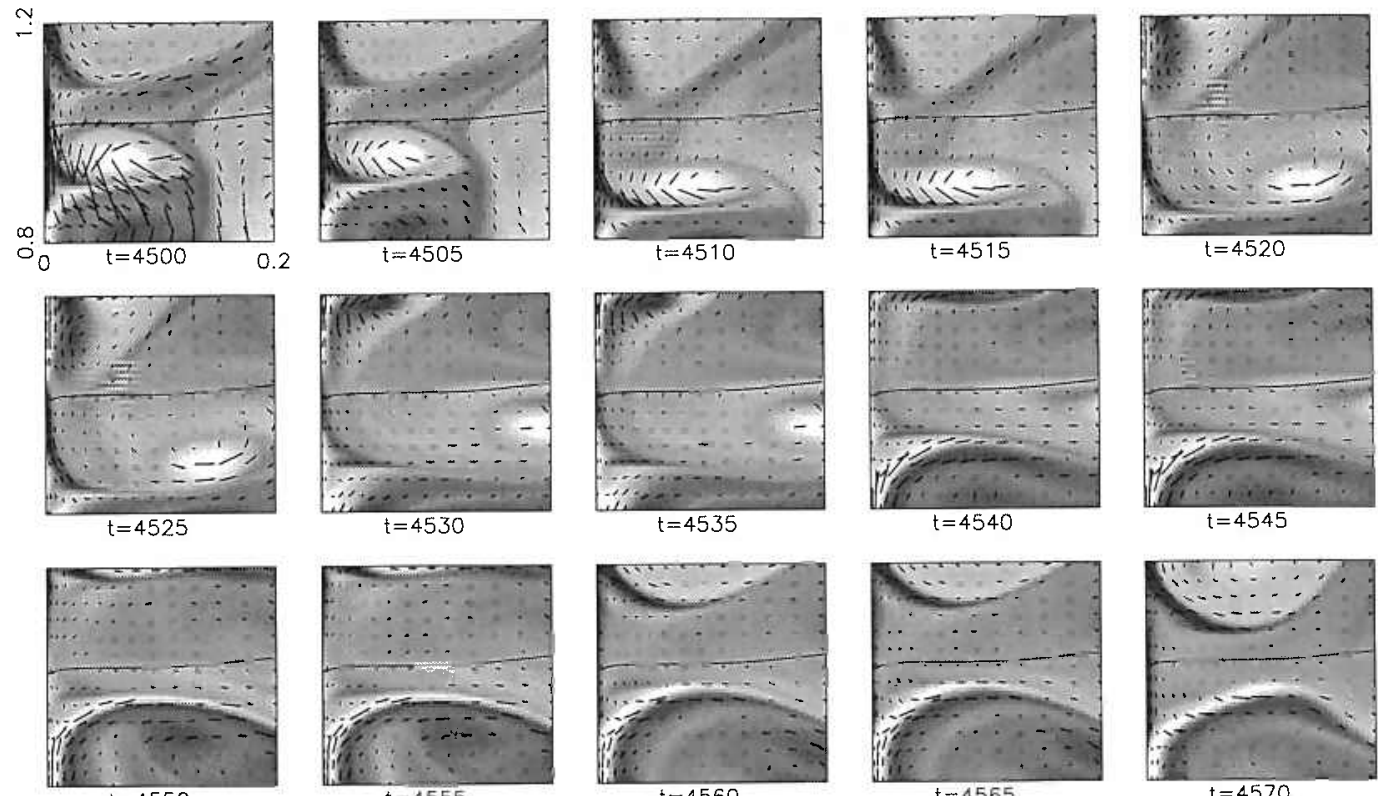

$t=4545$
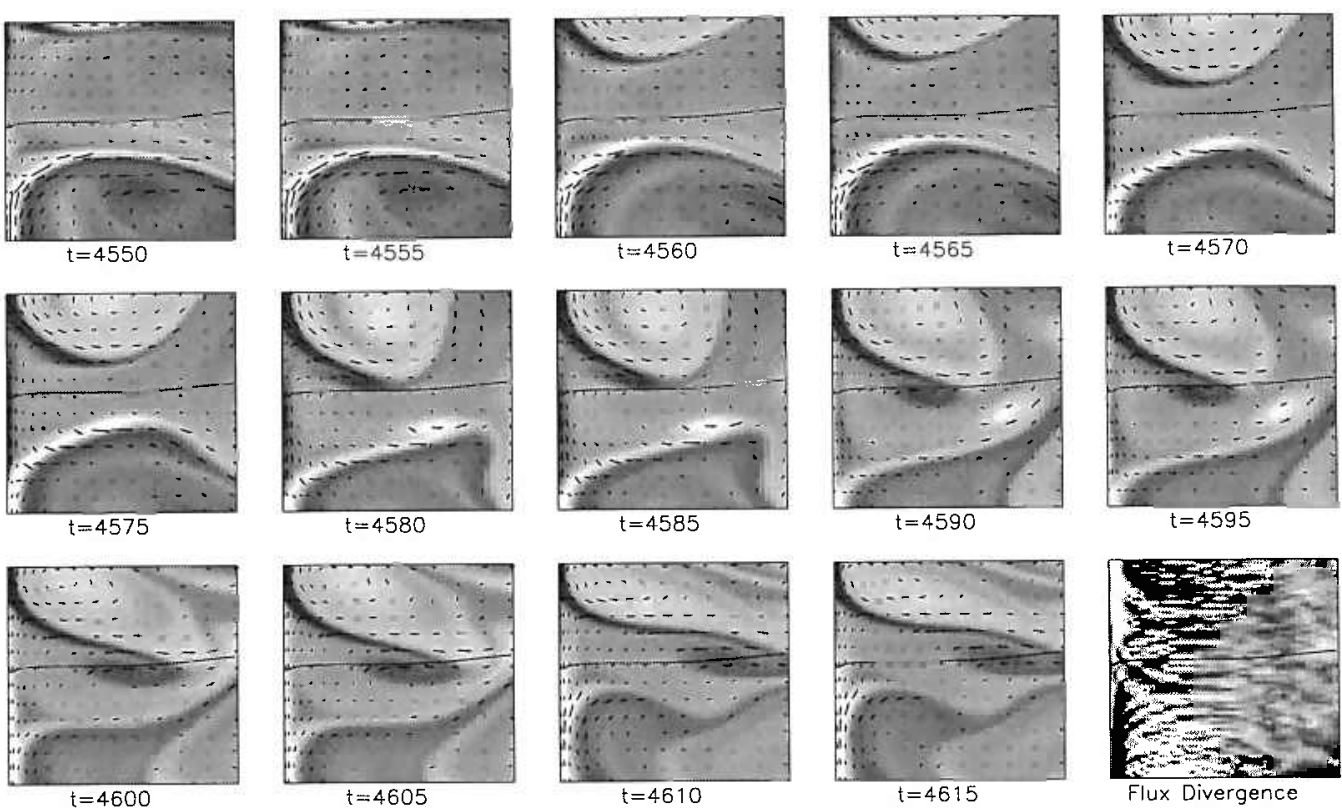

$t=4605$

$t=4610$

$t=4615$

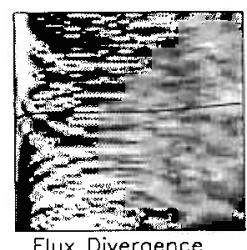

Figure 4-27: As in figure 4-25 but for the no-slip $\operatorname{Re}($ int $)=\operatorname{Re}(\mathrm{bdy})=5$ calculation. 


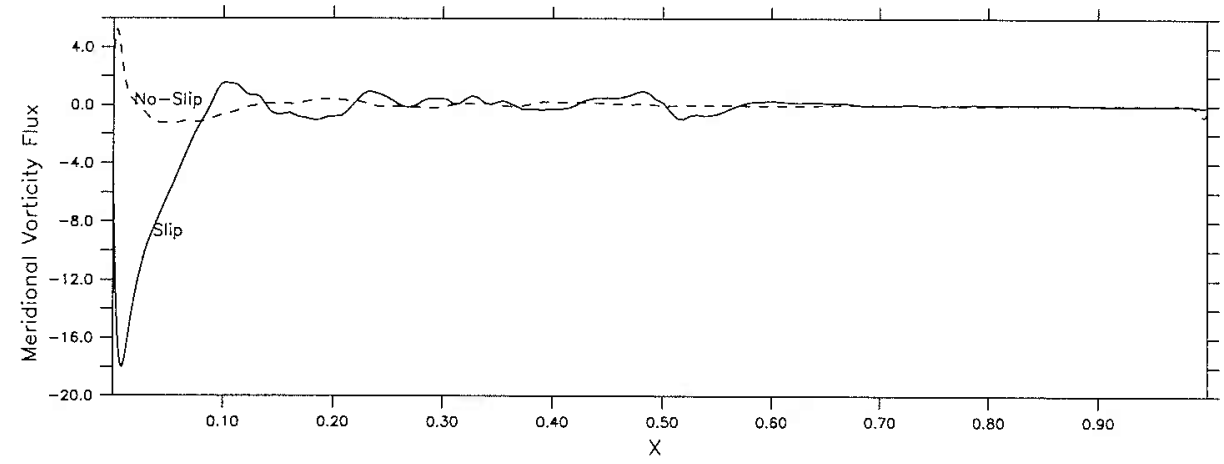

Figure 4-28: Direct estimates of eddy vorticity fluxes across $y=1$ for the $\operatorname{Re}($ int $)=5$, $\operatorname{Re}(\mathrm{bdy})=5$ calculation with no-slip boundary conditions and with slip boundary conditions. The mean fluxes across $y=1$ are negligibly small in comparison.

wanders backs and forth and the anomalies tend to be one sign or the other, then dissipative meandering can take place and can be quite strong. On the other hand, if the location is fixed or the relative vorticity anomalies tend to be cancellatory then dissipative meandering will be only a weak effect.

Obviously, a barotropic model of the ocean circulation does not represent the dynamics near the boundary very well. However, the two critical features of the dissipative meandering mechanism will likely still apply: 1) it must occur close to the boundary to allow for dissipation and 2) it requires significant meandering across the inter-gyre boundary. Thus, considering the Gulf Stream, the motion of the separation point should govern the amount of dissipative meandering. However, the Gulf Stream does not meander significantly north and south along the coast; it tends to separate near Cape Hatteras with a relatively fixed separation location. Some have even proposed that it is the topographic steering of Cape Hatteras that causes the separation at this location (for example, Marshall (2001)). These results tend to imply that the fixed location of the Gulf Stream will prevent significant dissipative meandering, and therefore this mechanism will be of limited applicability in the North Atlantic. 
In the real ocean, whether the relative vorticity of the eddies displaced across the boundary will be biased or not is an interesting question. The center of the Gulf Stream moves faster than its sides, which gives it vorticity of both signs as is the case in the no-slip calculations, although the shoreward side is not unmoving, so the cancellation is not as great. A more interesting question is if the sign bias introduced because eddies north of the Gulf Stream tend to be warm-core eddies, while those south of the Gulf stream tend to be cold-core eddies. Thus, by thermal wind, there may be a bias on relative vorticity imposed by the temperature gradient.

In summary, although the idea of eddies being able to transport vorticity from one gyre to the other is a compelling one, it is not guaranteed to occur. The method of inter-gyre vorticity exchange in the slip calculations presented here appears to be caused primarily by dissipative meandering. Because the irreversibility of this exchange is due to the direct action of friction, which is only very effective near the western boundary, the meandering must occur very close to the western boundary. Since no-slip boundary conditions prevent the jet from meandering along the coast and have a cancellatory vorticity structure in the boundary current, this effect is negligible in the no-slip calculations. Thus, in the real ocean the separation point of the boundary current must wander in time along the coast and there must be a bias of the relative vorticity anomalies advected by this meandering for there to be an inter-gyre flux of vorticity by dissipative meandering.

\subsubsection{Exposure to Opposing Wind Forcing}

Although the hypothesis of one gyre transiently experiencing the wind forcing of the other gyre seems distinct from dissipative meandering, in fact, these two hypotheses are closely related. In this section, I will demonstrate that transient exposure to the opposite wind-stress also implies a correlation in $\overline{v^{\prime} q^{\prime}}$. Unlike dissipative meandering, this mechanism does not require the eddy flux to be near the boundary, so it could contribute in both the no-slip and the slip calculations. However, it is clear from the 
small magnitude of the inter-gyre eddy flux in the no-slip case that this mechanism cannot be of primary importance in controlling the circulation strength.

Consider a parcel of fluid displaced across the zero wind-stress curl line. As the parcel experiences the wind forcing of the opposite sign of its native gyre, its relative vorticity magnitude will decrease (assuming its vorticity is of the sign typical of its gyre). If the the parcel returns to its original location at the same speed it left, its return results in a smaller eddy flux than its departure. Thus, a time-average inter-gyre eddy flux results.

This mechanism is obviously in operation in both the slip and no-slip calculations; even linear displacements of parcels by Rossby waves under a wind forcing would result in an eddy flux. However, the wind forcing's production of vorticity is simply not strong enough when compared to the rate of relative vorticity supplied by the boundary current. This is essentially because the wind stress curl changes direction smoothly at the inter-gyre boundary from negative in the subtropical gyre to positive in the subpolar, so the magnitude of the wind forcing is very small near the inter-gyre boundary. Therefore, only very large excursions of the jet would reach the region of strongest wind forcing (located near $y=0.5$ and $y=1.5$ in the double-gyre), and even then the excursions would need to cover a significant area in order to absorb a significant area-averaged change in vorticity (circulation). Thus, temporary exposure to the opposite wind forcing is likely to be a negligible contribution in this parameter range.

To prove this point, figure 4-29 demonstrates that removing the opposing subpolar wind-stress does not significantly affect the subtropical gyre circulation when no-slip boundary conditions are used. By comparing this result to the one shown in figure 42 , it is clear that the subtropical gyre is not significantly affected by the subpolar wind forcing in the case of no-slip boundary conditions.

In summary, transient exposure to opposing wind forcing experienced by displaced fluid parcels is negligible when compared to the dissipative meandering in the slip 


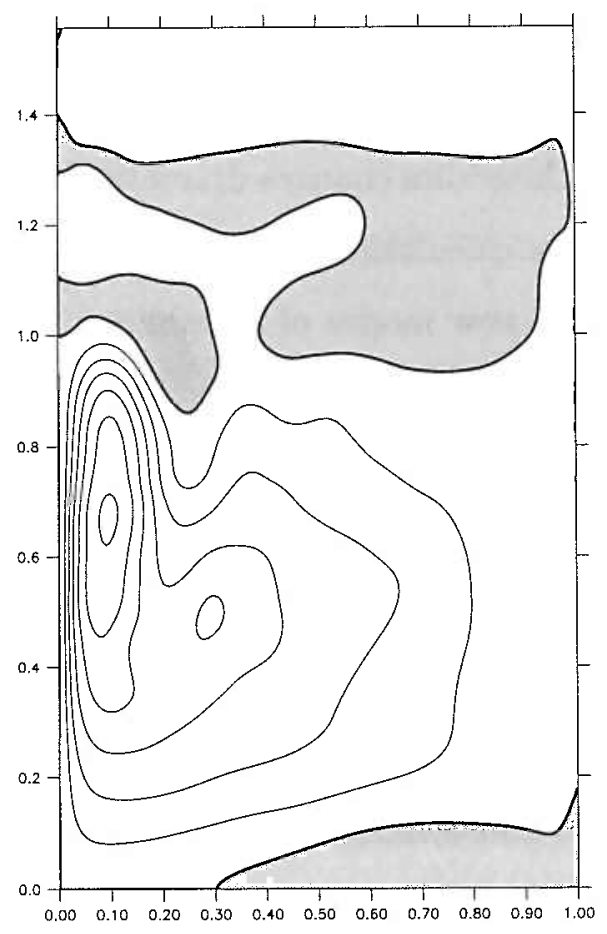

Figure 4-29: Contours of the time-mean streamfunction for a no-slip, $\operatorname{Re}(\mathrm{bdy})=5$, $\operatorname{Re}($ int $)=5$ calculation with single-gyre wind forcing between $y=0$ and 1 and no wind forcing for $y>1$. The northern boundary is located at $y=1.56$ as in the two-gyre calculations.

calculation. It is a weak effect in no-slip calculations, as it would require an intergyre vorticity flux, and no-slip calculations without opposing wind stress are relatively unchanged. 


\subsubsection{Sinuous Modes: Breaking the Initial Symmetry of the Double-Gyre}

The final hypothesis that I present in this section is novel. Consider that it is highly likely that the structure of the eddies changes dramatically when the northern boundary present in the single-gyre calculation is removed upon addition of a second gyre. By the introduction of these new modes of variability, the eddy flux of vorticity to the frictional sublayer (and the inter-gyre eddy flux as well) can be made significantly more efficient, destroying the recirculations and thereby controlling the circulation strength. As we have seen in section 3.7, the frictional sublayer is capable of more easily handling vorticity flux when it comes in more than one form; apparently the same effect applies here.

It is well-known that the sinuous instabilities of jets have significantly larger growth rates and therefore effect a much larger eddy advection than varicose modes. Sinuous modes are even in the streamfunction by definition (see figure 4-30), so they cannot have corresponding instabilities in the single-gyre because the northern boundary requires $\psi=0$. Only the slower-growing varicose modes of the jet can have cousins in the single-gyre. Actually, the presence of either a northern boundary with slip conditions or exact antisymmetry between the two gyres allows for only the weaker varicose instabilities to form. Therefore, removing the northern boundary or breaking the exact antisymmetry of the double-gyre leads to an enormous increase in the effectiveness of eddy activity. This result is well known for the instabilities of non-rotating two-dimensional jets (for example, Drazin and Reid (1981), Lamb (1932)). Recently, Balmforth and Piccolo (2001) demonstrated this result for instabilities of the Bickley jet with rotating, barotropic dynamics identical to those used in this dissertation.

Of course, in all of the calculations, instabilities which occur in the western boundary current before separation are present and active (although much more active in no-slip calculations than in slip). The single-gyre and antisymmetric calculations 

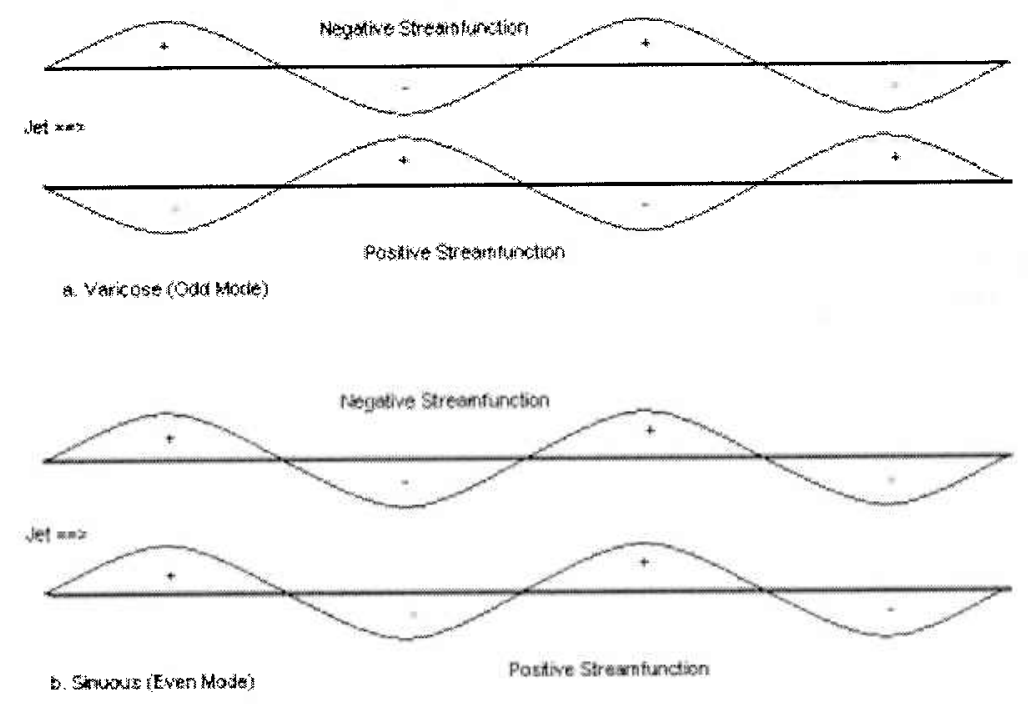

Figure 4-30: Schematic of symmetry properties of sinuous and varicose modes of instability on a jet. The thick lines represent initial jet streamlines, the thin lines represent streamlines after the instability has grown somewhat. The pluses and minuses denote streamfunction anomalies.

have these western modes as well as the varicose modes. ${ }^{6}$ In section 3.4 , most of the large variance EOFs of relative vorticity seemed to represent western modes of instability, and the eddy flux divergence patterns are typically contained near the western boundary. Thus, it is likely that these modes are more important than the varicose ones. In the two-gyre and double-gyre calculations, relative vorticity EOFs reveal both sinuous modes and western modes are present with roughly equal variances (not shown).

Figure 4-31 demonstrates that like the single-gyre, the exactly antisymmetric double-gyre calculation has difficulty in controlling its circulation strength. This figure shows that in the exactly antisymmetric double-gyre calculation started from exactly antisymmetric initial conditions, the solution evolves with exact antisymme-

\footnotetext{
${ }^{6}$ Here I mean the single-gyre as used throughout this dissertation with slip northern and southern boundaries. Obviously, a no-slip northern boundary condition would make the northern boundary quite unlike a neighboring gyre's jet.
} 


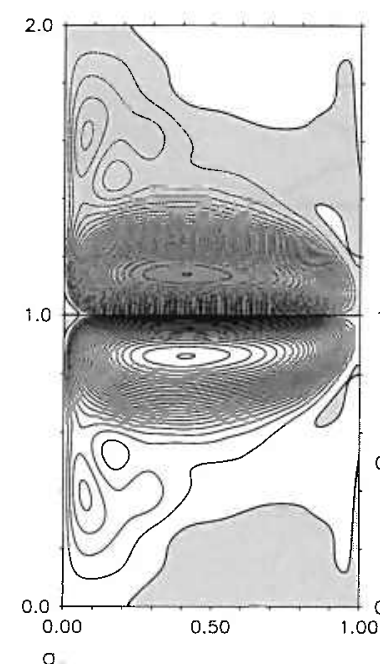

o

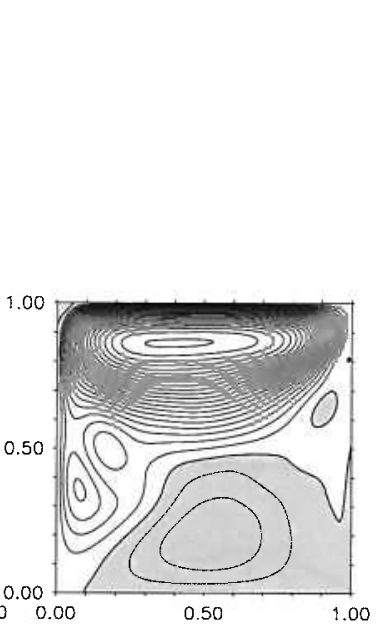

b.

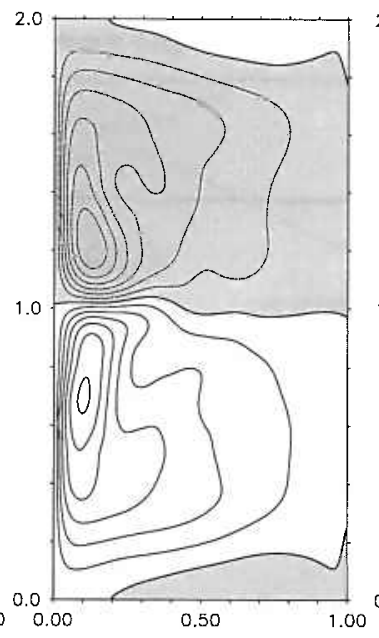

c.

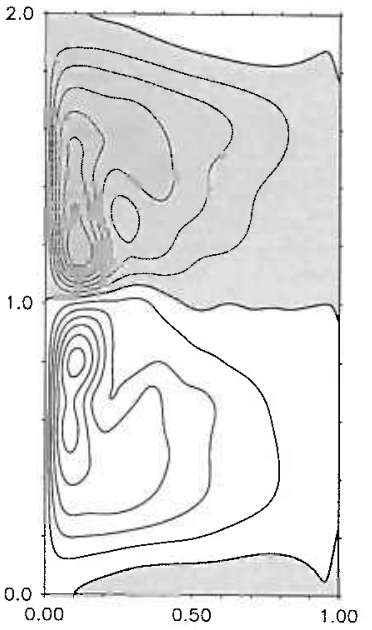

Figure 4-31: Comparison of the time-mean streamfunction for no-slip, $\operatorname{Re}($ bdy $)=5$, $\operatorname{Re}($ int $)=5$ calculations. (a) and (c) are averages over different time intervals for a calculation with an at rest (symmetric) initial condition. (a) is the time-mean over an interval just before exact antisymmetry is broken by numerical errors ( $t=2000$ to 2750 ), and (c) is averaged over the time-interval $t=8990$ to 13710 . (b) shows the time-mean streamfunction for the single-gyre calculation. (d) shows the time-mean streamfunction for the calculation with symmetry-breaking initial conditions. The contour interval is 0.2 , regions of negative streamfunction are shaded.

try for a time. Eventually (at about time 2500) numerical errors break the unstable equilibrium of antisymmetry. However, before the symmetry breaks, the circulation had grown very strong, with approximately four times more mass transport than it settles out at after the antisymmetry is broken.

In fact, before the symmetry breaks the calculation appears to have been on the way to making two mirror copies of the single-gyre calculation. The subtropical gyre in antisymmetric equilibrium solution strongly resembles the single-gyre calculation (figure 4-31c) at the same Reynolds number. Differences are easily attributed to the incomplete spin-up before the symmetry broke. As shown in figure 3-11, the single-gyre calculation in figure 4-31c took approximately 3000 time units to spin-up.

After the antisymmetry is broken, the solution reaches a new equilibrium state 
which closely resembles the double-gyre solution started with symmetry-breaking initial conditions (figure 4-31d), although it takes a very long time for the strong circulation of the exactly antisymmetric circulation to be slowed.

Figure 4-32 shows that the resemblance between the time-mean streamfunctions is not trivial. The eddy flux divergences of the broken symmetry calculations are very similar, and the flux divergences of the antisymmetric average and that of the single-gyre are also similar in form.

Many of the differences in the flux divergences between the antisymmetric spin-up and the single-gyre are due to the fact that the antisymmetric average is not fully spun-up. Also, the time-average of the antisymmetric average was not performed over a very long time interval when compared to the averaging interval of the single-gyre calculation (750 time units as opposed to 5000 time units).

Apparently, the eddies present in the single-gyre strongly resemble those of the exactly antisymmetric double-gyre. The removal crisis and reversal in eddy flux divergence at the boundary near $y=0.5$ (which was used as an indication of insufficient boundary viscosity in section 2.3.2) is present in both calculations, although it is not as strong in figure 4-32a. Since the reversal in eddy flux divergence is present in the antisymmetric double-gyre, it is likely that as in the single-gyre case, boundaryenhanced viscosity would be required to bring the antisymmetric circulation under control without breaking the antisymmetry. Even details such as the hook-like pattern in divergence where the boundary current joins the jet are reproduced.

Figure 4-33 compares snapshots of relative vorticity from the single-gyre calculation to those of the subtropical region of the double-gyre calculation before the antisymmetry is broken. While many of the eddying features are shared, some are not. One prime example is the small, intense negative vorticity eddy that chases around the northern part of the recirculation gyre and then enters the gyre in the antisymmetric solution. No features of this kind are present in the snapshots of the single-gyre shown, and I have not found many by examining movies of other times 

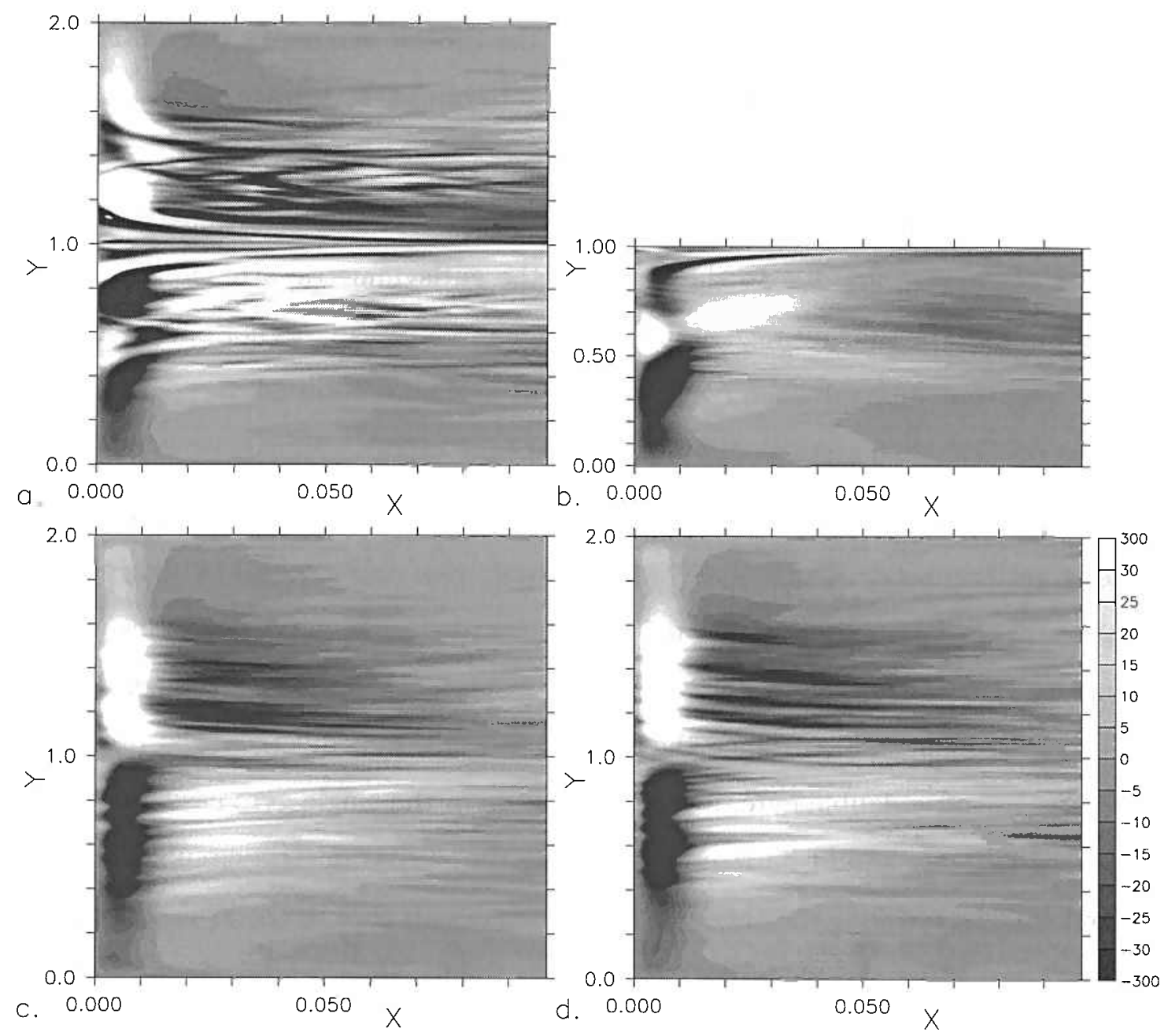

Figure 4-32: Comparison of the time-mean eddy flux convergence in a region near the western boundary for the no-slip, $\operatorname{Re}(\mathrm{bdy})=\operatorname{Re}(\mathrm{int})=5$ calculations shown in figure 431 (with the same figure lettering). Some smoothing was applied to (a) and (c). 

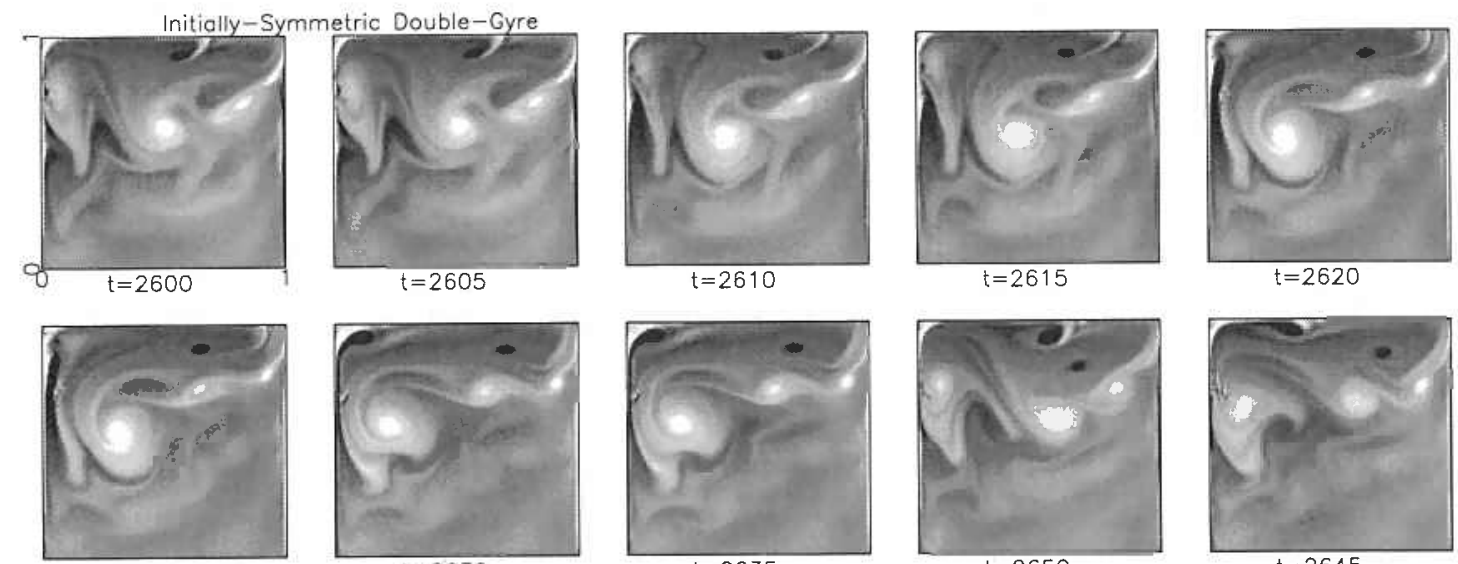

$t=2605$

$t=2610$

$t=2615$

$t=2620$

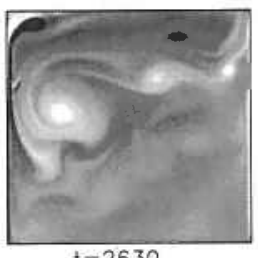

$t=2630$

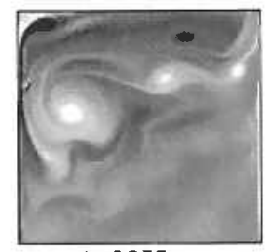

$t=2635$

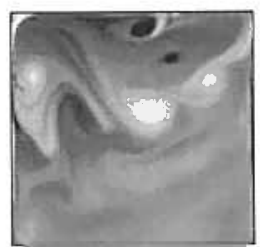

$t=2650$

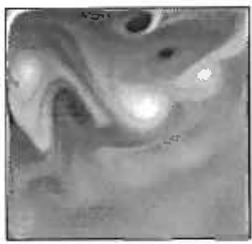

$t=2650$

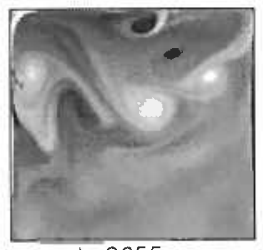

$t=2655$

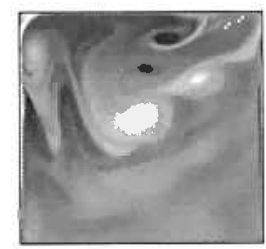

$t=2660$

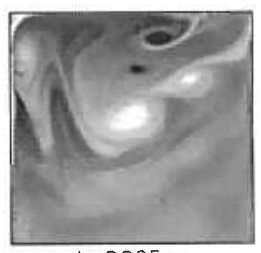

$t=2665$

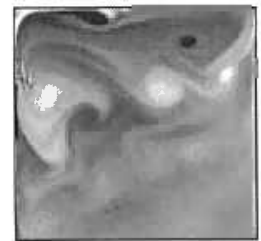

$t=2645$
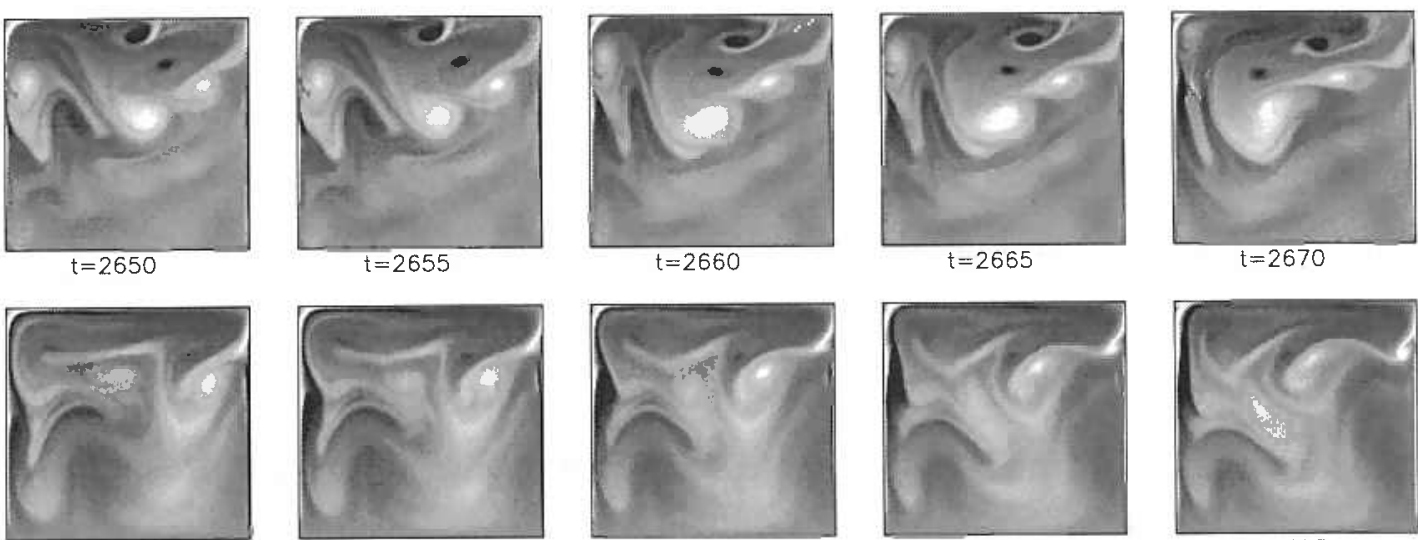

$t=7575$

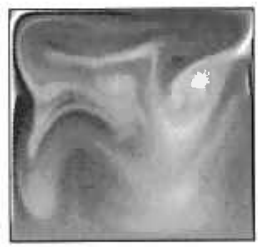

$t=7580$

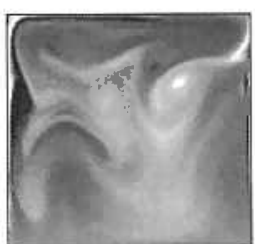

$t=7585$

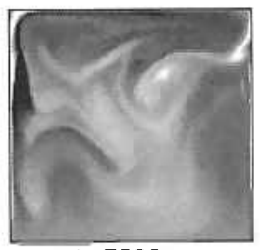

$t=7590$

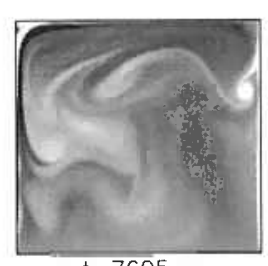

$t=7605$

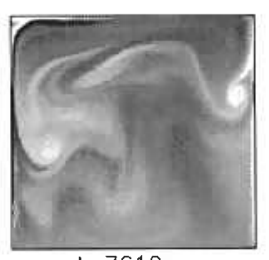

$t=7610$

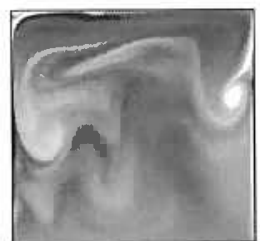

$t=7615$

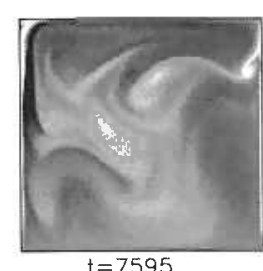

$t=7595$

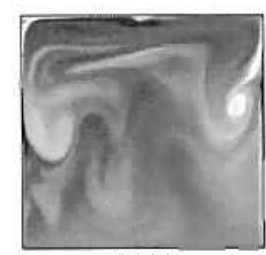

$t=7620$

Figure 4-33: Snapshots of relative vorticity in the subtropical portion ( $y$ between 0 and 1) of the double-gyre calculation begun with symmetric initial conditions before symmetry is broken (upper three rows) and snapshots of the single-gyre calculation for comparison (bottom two rows). Shading scheme is the same for the two calculations (lighter=more positive vorticity, darker=more negative). 
and other single-gyre calculations. These small eddies with this trajectory seem to be quite common in the antisymmetric double-gyre, however. Thus, the double-gyre, though similar to the single-gyre before the breakdown of antisymmetry, is not precisely the same as the single-gyre.

Figure 4-34 shows the breakdown of the jet separating the gyres under the action of the sinuous modes. Obviously, the exactly antisymmetric equilibrium is an unstable one. The new modes of instability which are introduced when the antisymmetry is broken drastically change the character of the solution, even though at first there is only a tiny asymmetry present from the numerical errors.

One might wonder where the vorticity from the recirculation goes. First, note that this not relevant to understanding the equilibrium state. In equilibrium, vorticity is constantly supplied and constantly removed through the boundaries. To reach equilibrium, a balance is only necessary between the inflow and the outflow of vorticity. Whatever storage of vorticity is kept in the basin is irrelevant to this budget, except that the amount in storage affects the rate of outflow. In the single-gyre, the amount of vorticity contained in the basin has two effects. 1) It can increase the gradient of the vorticity right at the boundary, enhancing frictional removal, or 2) it can fuel the instabilities which produce eddies which in turn transport vorticity to the frictional sublayer. Apparently, the sinuous modes are a mechanism which makes the same outflow of vorticity to the frictional sublayer occur with a much smaller requirement of fuel from the storage of vorticity.

Although the removal of the vorticity from the recirculation gyre is not relevant to the equilibrium state, the transient problem is also of interest. Although these modes are efficient at transporting vorticity to the frictional sublayer, it still takes quite a while for all of the vorticity which had built up in the recirculation gyre to be removed from the basin. While the single-gyre spins up in approximately 3000 time units and the antisymmetric equilibrium takes a similar amount of time, it takes approximately twice that long to remove the vorticity from the basin. Over this time 

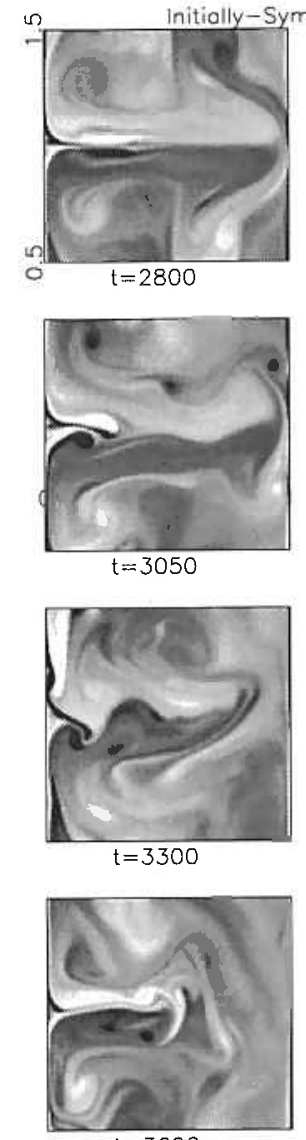

$t=3600$

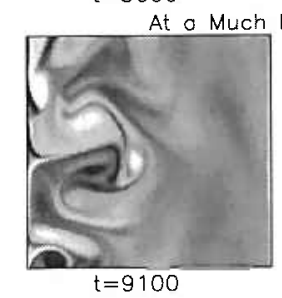

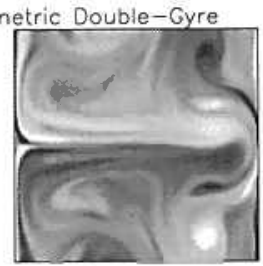

$t=2850$
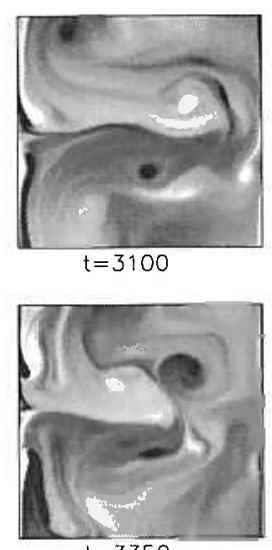

$t=3350$

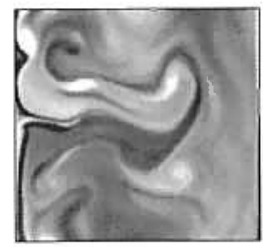

$t=3650$

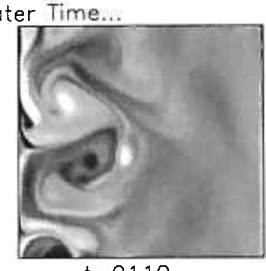

$t=9110$

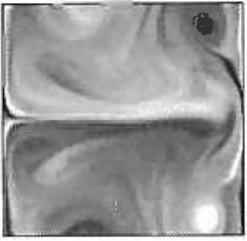

$t=2900$

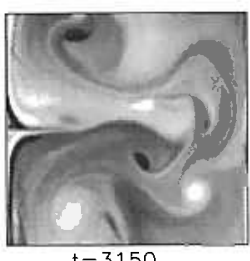

$t=3150$

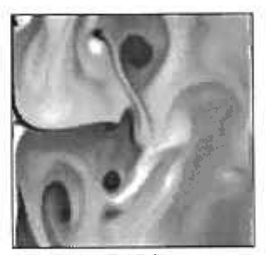

$t=3400$

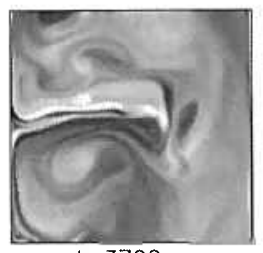

$t=3700$

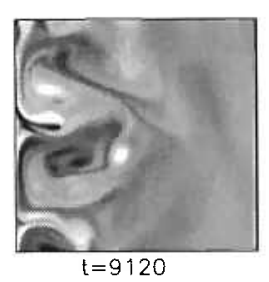

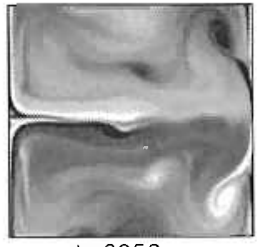

$t=2950$

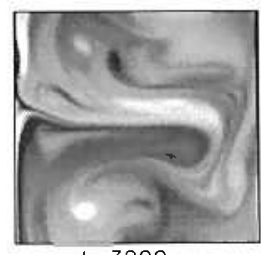

$t=3200$

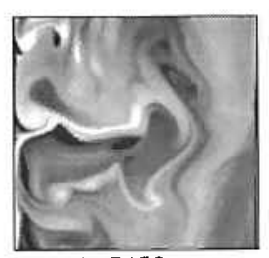

$t=3450$

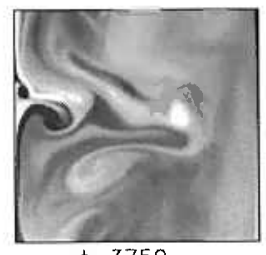

$t=3750$

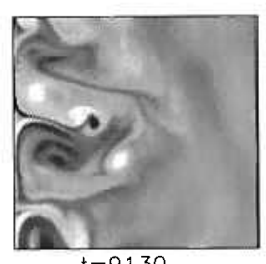

$t=9130$

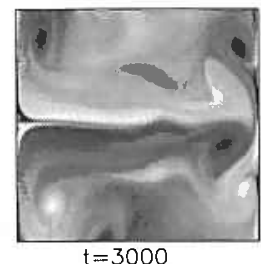

$t=3000$

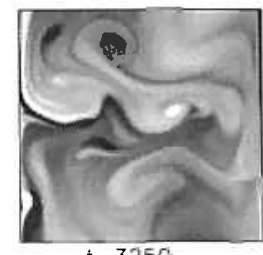

$\mathrm{t}=325 \mathrm{C}$

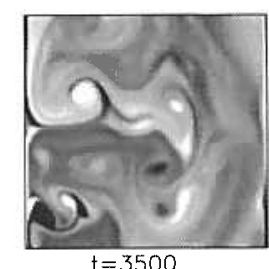

$t=3500$

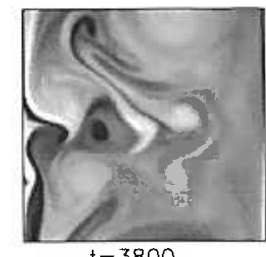

$t=3800$

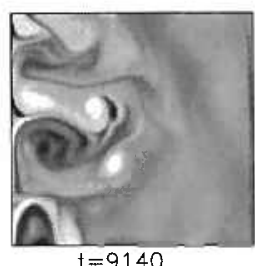

Figure 4-34: Snapshots of relative vorticity in the region surrounding the jet ( $y$ between 0.5 and 1.5) of the double-gyre calculation begun with symmetric initial conditions as symmetry is broken (upper four rows) and snapshots after the circulation strength has reached equilibrium (bottom row). Shading scheme is the same as in figure 4-33. 
interval, the eddies must transport a flux of vorticity to the frictional sublayer whose magnitude is in excess of the wind's forcing for frictional removal.

During this spin-down process there is some inter-gyre flux of vorticity, but more of the spin-down is accomplished with eddy fluxes to the frictional sublayer within the same gyre. Integrating the eddy flux divergence for $y<1$ and averaging over the time interval 3000 to 4000 yields a modest average inter-gyre vorticity flux of 0.04 out of the wind input of 0.64 . The average frictional removal of vorticity from the $y<1$ region over the same time interval is 0.73 , so approximately twice as much of the excess vorticity is removed frictionally during this time. Of course, it is difficult to be sure that this average is accurate, as there is insufficient time to be sure of the behavior of the eddies. Averaging over a longer interval (3000 to 5000), the frictional removal is 0.71 and the inter-gyre eddy flux is 0.03 . The reduction in the magnitudes of average removal rate by both friction and inter-gyre eddy flux with a relatively constant ratio between the rates is consistent with an exponential decay of the recirculation gyre's vorticity from the basin.

In section 4.3 , the artificiality of the subpolar and subtropical boundary currents being equally matched in the double-gyre model was pointed out. Figure 4-31 accentuates another problem with the exact symmetry of the double-gyre. If the initial condition is exactly antisymmetric about $y=1$ and the model is also antisymmetric, the solution converges toward the antisymmetric equilibrium solution. This solution has enormous mass transport, and there is no reason to expect it to exist in any natural situation. For this reason, all of the double-gyre calculations in this dissertation were initialized with a non-zero initial state (except for figures 4-31 and 4-32, obviously). This made the spin-up time much more reasonable than waiting for the numerical errors to break the exact antisymmetry.

Slip calculations have a similar symmetry-breaking problem as the no-slip calculations. I have performed similar calculations as in figure 4-31 with a slip calculation and found similar results. The sinuous modes are required for control of the circula- 


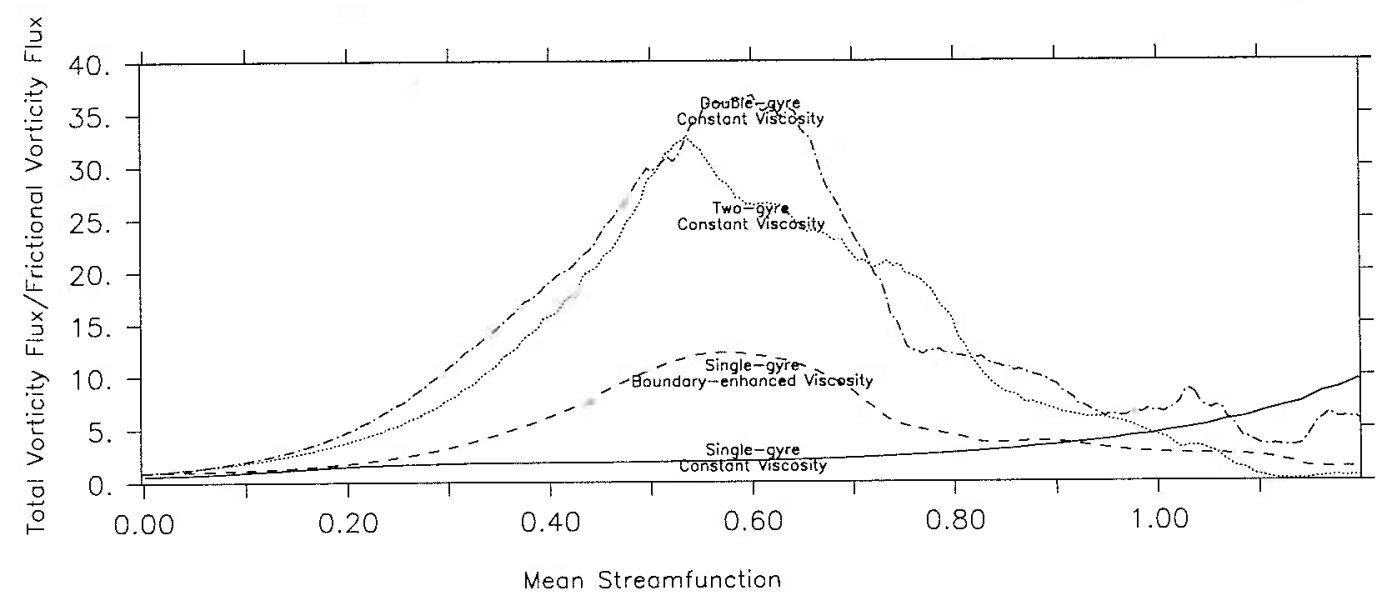

Figure 4-35: This figure shows the ratio of the total vorticity flux across a mean streamline to the frictional flux across a mean streamline as a function of mean streamfunction. The ratio is shown for the no-slip $\operatorname{Re}($ bdy $)=\operatorname{Re}(\mathrm{int})=5$ calculations (single, double, and two-gyre) and the $\operatorname{Re}(\mathrm{bdy})=0.25, \operatorname{Re}(\mathrm{int})=5$ single-gyre, no-slip calculation.

tion strength there as well. A study by Le Provost and Verron (1987) addresses the changes in the jet with slip conditions, but their calculations were too viscous to have inertially-dominated equilibria.

Another way to describe this effect is to say that the eddies are more efficient in transporting vorticity across mean streamlines when sinuous modes are present than when they are not. In figures $2-10$ and 3-4, a ratio of the total vorticity flux across mean streamlines to the frictional flux across mean streamlines (a construct similar to the Nusselt number) was shown to be a good indicator of the efficiency of eddy transport across mean streamlines. Using this diagnostic, it was demonstrated that the eddy flux is made more efficient by introduction of boundary-enhanced viscosity, and that the homoparic pair member with larger Re(int) had more efficient vorticity transport.

In figure $4-35$, the ratio of the total vorticity flux across mean streamlines to the frictional vorticity flux for single-gyre constant viscosity and boundary-enhanced 
viscosity calculations, as well as constant viscosity two-gyre and double-gyre calculations. This figure shows that although the boundary-enhanced viscosity increased the efficiency of cross streamline vorticity fluxes somewhat (by about a factor of 10), the introduction of the sinuous modes increases the efficiency of the vorticity fluxes dramatically (by about a factor of 30). Thus, in the presence of sinuous modes the vorticity tends not to pool in the recirculation gyre causing it to enlarge, instead the efficient eddy vorticity flux to the boundary allowed by the sinuous modes prevents its growth.

By comparing the frictional flux through the western boundary in the single-gyre no-slip calculations in figure 2-7 to the flux through the western boundary in the no-slip, two-gyre calculation shown on the left of figure 4-17, we see that the vorticity removal is quite different when the northern boundary is removed and sinuous modes are present. As mentioned above, the frictional flux through the western boundary in the no-slip, two-gyre calculation shown in figure 4-17 reveals that the wind input is removed at nearly the same latitude where it is input by the wind. In the single-gyre calculations, however, even in the western-intensified solution the vorticity budget is satisfied by removal at latitudes different from the input latitude. Thus, the increased efficiency of the eddy flux to the frictional sublayer with the introduction of the sinuous modes makes the removal of vorticity occur at the same latitude as the input (at this Reynolds number), just as in the linear Munk solution. However, we know that unlike the linear Munk solution, the interior mean flow streamlines never enter the region of frictional influence. The eddies are responsible for fluxing the vorticity across these streamlines. The double-gyre, no-slip calculations have a similar removal of vorticity at the same latitude as the input.

Although it is beyond the scope of this thesis to do a full instability analysis of these solutions, some results can be shown that are illuminating as to the structure and function of the sinuous and varicose instabilities in the slip and no-slip calculations. Figure 4-36 is an attempt to separate the effects of the odd modes of variability 
from the even modes' effects. This figure is generated in the following way. Consider the streamfunction perturbation from the mean, $\psi^{\prime}$. It will generally be composed of parts which are odd about the jet and parts which are even. Although the dynamical source of these parts is not easily directly determined, if we assume that the jet is located exactly at $y=1$ the perturbation streamfunction is easily split into odd and even parts in the case of the double-gyre. To wit,

$$
\begin{aligned}
\psi_{o}^{\prime} & =\frac{\psi^{\prime}(y)-\psi^{\prime}(2-y)}{2} \\
\psi_{e}^{\prime} & =\frac{\psi^{\prime}(y)+\psi^{\prime}(2-y)}{2}
\end{aligned}
$$

Likewise for the relative vorticity perturbation:

$$
\begin{aligned}
& \zeta_{o}^{\prime}=\nabla^{2} \psi_{o}^{\prime}=\frac{\nabla^{2} \psi^{\prime}(y)-\nabla^{2} \psi^{\prime}(2-y)}{2}=\frac{\zeta^{\prime}(y)-\zeta^{\prime}(2-y)}{2} \\
& \zeta_{e}^{\prime}=\nabla^{2} \psi_{e}^{\prime}=\frac{\nabla^{2} \psi^{\prime}(y)+\nabla^{2} \psi^{\prime}(2-y)}{2}=\frac{\zeta^{\prime}(y)+\zeta^{\prime}(2-y)}{2} .
\end{aligned}
$$

Now it is easy to invert these relations and use this result to determine the dependencies of the eddy vorticity flux divergence.

$$
\begin{gathered}
\psi^{\prime}=\psi_{o}^{\prime}+\psi_{e}^{\prime} \\
\zeta^{\prime}=\zeta_{o}^{\prime}+\zeta_{e}^{\prime} \\
\overline{J\left(\psi^{\prime}, \zeta^{\prime}\right)}=\overline{J\left(\psi_{o}^{\prime}, \zeta_{o}^{\prime}\right)}+\overline{J\left(\psi_{e}^{\prime}, \zeta_{e}^{\prime}\right)}+\overline{J\left(\psi_{o}^{\prime}, \zeta_{e}^{\prime}\right)}+\overline{J\left(\psi_{e}^{\prime}, \zeta_{o}^{\prime}\right)}
\end{gathered}
$$

These eddy flux divergences represent the self-advection of varicose (odd) variability, the self-advection of sinuous (even) variability, the advection of sinuous variability by varicose variability, and the advection of varicose variability by sinuous variability. It is these terms which are plotted in figure 4-36.

It should be clear that this is an imperfect separation of the modes' effects; obviously the jet is not exactly at $y=1$ and the presence of interaction terms indicates 

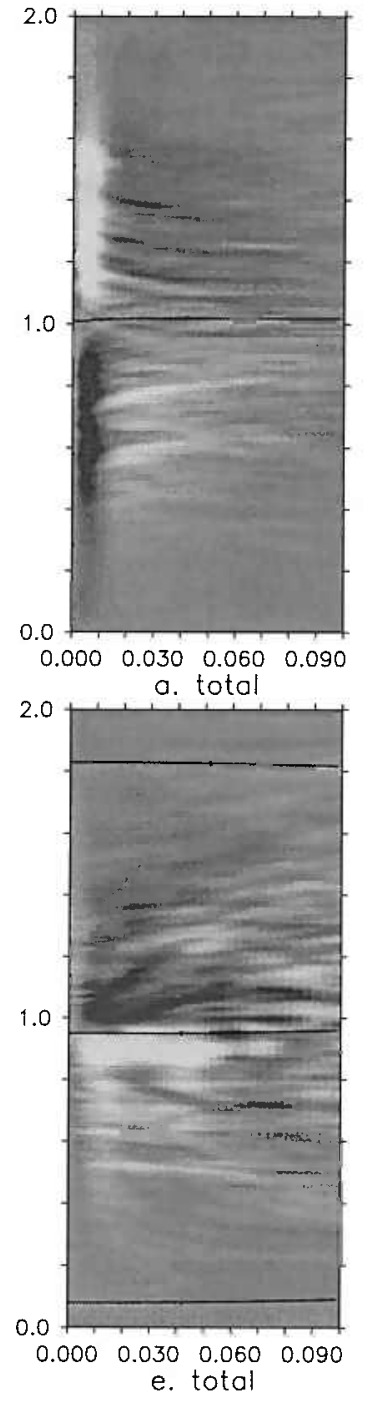
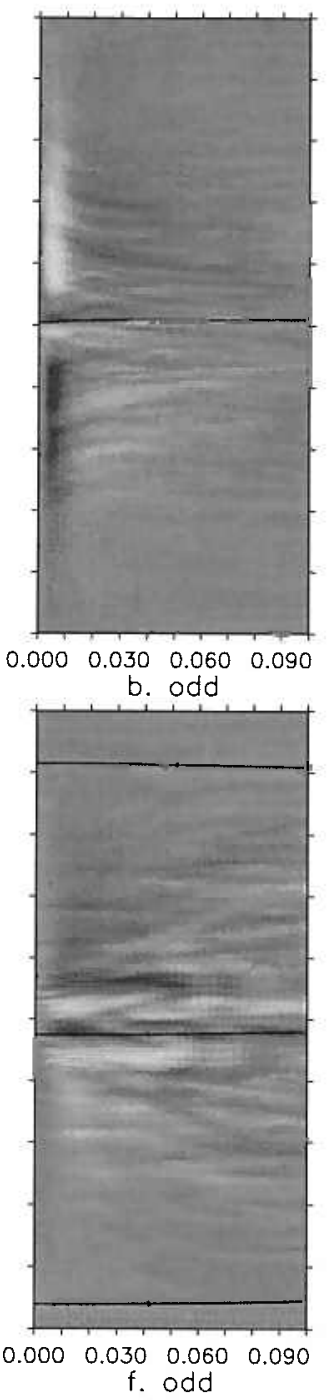

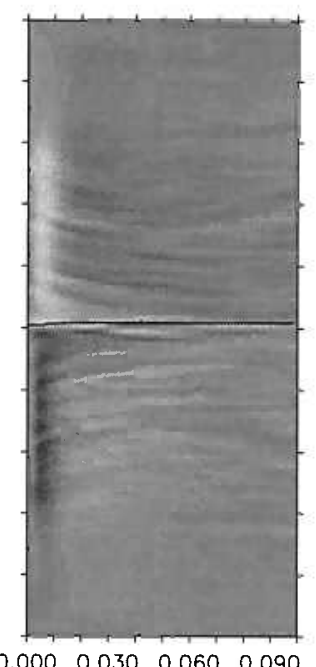

$\begin{array}{lllll}0.000 & 0.030 & 0.060 & 0.090\end{array}$ c. even

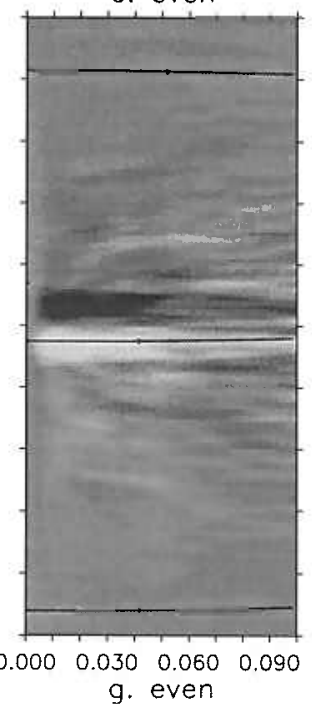

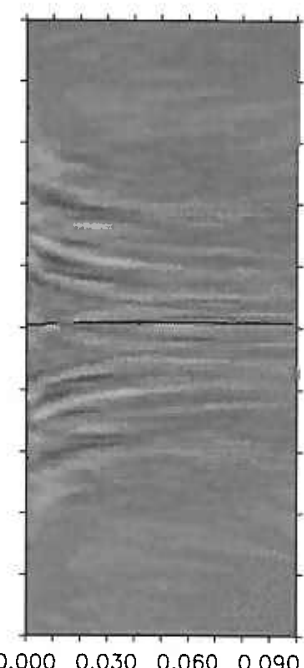

d. interact

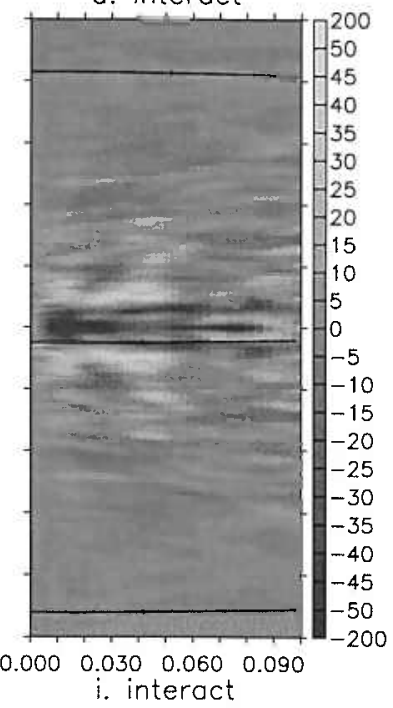

Figure 4-36: Comparison of the eddy flux convergences in a region near the western boundary from eddies with different symmetry about $y=1$. (a-d) are for the $\operatorname{Re}(b d y)=\operatorname{Re}($ int $)=5$, no-slip calculation. $(e-h)$ are for the $\operatorname{Re}(b d y)=\operatorname{Re}($ int $)=5$ slip calculation. (a) and (e) are the total flux convergence. The rest of the images are other flux convergences: (b) and (f) are from self-advection of varicose (odd) variability, (c) and (g) are from self-advection of sinuous (even) variability, and (d) and (h) are from the interactions between varicose and sinuous variability (see equation 4.4). The streamline dividing the gyres is added for reference. 
that the even and odd modes are not independent. Also, no attempt is made to remove or address the effects of the western modes.

From comparing figure 4-36e and 4-36g, it is clear that the sinuous mode performs the bulk of the inter-gyre flux of vorticity in the slip calculation. In fact, the mechanism of dissipative meandering requires a sinuous mode. Only a sinuous mode can displace the jet alternately north and south. The varicose modes in the slip calculation do a small amount of mixing to either side of the jet, but contribute little to the inter-gyre flux of vorticity. Very little of the eddy flux divergence is concentrated near the western boundary, so the western modes are not very active if they are present (note that they were not present in the single-gyre calculation with $\operatorname{Re}(\mathrm{bdy})=2.5$, $\operatorname{Re}(\mathrm{int})=2.5)$. The sinuous modes' self-advection accounts for 0.40 of the 0.56 intergyre flux of vorticity in this calculation. The even and odd modes contribute similar (small) amounts of eddy flux to the frictional sublayer.

In the case of no-slip boundary conditions (figures 4-36(a-d)) none of the modes effect a significant inter-gyre flux of vorticity. It appears that the even and odd modes share equally in transporting the vorticity to the frictional sublayer where it is removed from the basin. Importantly, however, the removal crisis breakdown in the boundary current that occurs in the single-gyre and when only the varicose and western modes are present (figure 4-32) is not present in any of the flux divergences here. Therefore, the inclusion of the sinuous modes somehow prevents the breakdown, which makes the delivery of vorticity to the frictional sublayer much more efficient.

This gain in efficiency is present whenever the northern boundary is removed from a no-slip calculation. It is present in the double-gyre and two-gyre calculations (figures 4-2 and 4-4), and it is even present in the case of a subpolar region without wind forcing (figure 4-29). Only in exactly antisymmetric calculations or calculations with a northern boundary is the efficiency lost, along with the sinuous modes.

The gain in efficiency can be compared to the gain in efficiency that was seen in section 3.7 with the addition of a small amount of bottom friction. Although the 
bottom friction can not flux the vorticity out of the basin at the no-slip boundaries, it can deliver it to the frictional sublayer. The increased efficiency meant that the frictional removal could take place with a weaker mean circulation strength. There, it was unclear whether it was the bottom friction's effective transport of vorticity out of the recirculation gyre or its stabilization of the eddies which prevented the removal crisis.

Although it remains unclear exactly how the gain in efficiency is made by the introduction of sinuous modes, consider the following: since the inter-gyre flux of vorticity is negligible for no-slip boundary conditions, it is the eddy flux across mean streamlines to the western boundary which is important. Thus, what is critically different about the sinuous and varicose and western modes is their ability to strip vorticity out of the recirculation gyre and transport it to the boundary in a form which is easily dissipated. In the single-gyre calculations the varicose variability does not grow quickly enough to remove vorticity from the recirculation gyre, and the western modes cannot reach much of the recirculation gyre as it extends to the east.

It is easy to believe that the sinuous modes would be very effective in mixing across the mean streamlines of the recirculation gyre. Both Rogerson et al. (1999) and Balmforth and Piccolo (2001) found cat's eye formations with the hyperbolic points that cause intense mixing were associated with the growth of sinuous modes on the jet. Le Provost and Verron (1987) showed that these modes control the extent of the jet even in calculations with large enough bottom friction to prevent western boundary instabilities. Also, Balmforth and Piccolo (2001) showed that the growth rates of sinuous modes similar to those here were many times in excess of those for the varicose modes. Since the eddy advection is directly related to the growth rate for wavelike disturbances, this seems to indicate that the sinuous modes should be more effective at mixing in the basin interior.

The frictional sublayer is able to handle the vorticity flux from both the sinuous modes and the western and varicose modes more smoothly than the vorticity flux 
from just the western and varicose modes. The removal crisis from section 2.3 .2 and its reversal in the eddy flux divergence in the frictional sublayer is clearly present in figure 4-32(a-b). It is plain that with only the western and varicose eddies present, the frictional sublayer behaves the same as it does in the single-gyre calculation. However, when the sinuous modes appear, the reversal of sign in eddy flux divergence in the frictional sublayer disappears, and the frictional sublayer begins smoothly removing vorticity from the basin all along the boundary. Since there is no inter-gyre vorticity flux in any of these calculations, the magnitude of the removal of vorticity never changes, but including the sinuous modes makes the transport to the frictional sublayer possible with a significantly weaker mean flow. Recall that it is changes in the mean flow which are required for frictional removal, as the friction on the eddies averages to zero and thus does not appear in the Reynolds-averaged vorticity equation (equation 2.7.

This returns the discussion to the possibility that the recirculation gyre may be partially responsible for the removal crisis. The reversal in the sign of the eddy flux occurs near the region of the boundary current where the recirculation gyre approaches. In section 2.3.2 I presented two possible mechanisms where growth of the recirculation gyre could initiate the removal crisis. It could affect the eddy fluxes to the frictional sublayer, or it could cause a meander in the boundary current and produce a reversal in the sign of the friction flux convergence. Obviously, if the eddies are not able to remove enough vorticity from the recirculation gyre, it will continue to gain vorticity and grow in size and strength. Hypothetically, instead of the recirculation gyre being the result of a removal crisis, it could be a cause. In the single-gyre, the only way to energize the eddies and prevent a recirculation from growing was to enhance the viscosity near the boundary. Once the sinuous modes are present, the recirculation gyre can be reduced by the more efficient eddy activity which prevents the storage of vorticity in these interior streamlines. If the recirculation is causing the removal crisis, then its removal would remove the crisis. 
Despite the large growth rate of the sinuous modes and the mixing due to the associated cat's eyes in the calculations, by separating the even and odd modes in the no-slip calculation as shown in figure 4-36 and budgeting the flux through mean streamlines, I find that the fluxes across interior mean streamlines due to the even (sinuous) modes are never greater than $40 \%$ larger than those due to the western and varicose modes for equilibrated solutions. The interaction modes' fluxes are negligible. Perhaps this indicates that once the antisymmetry is broken the western modes are no longer antisymmetric and they contribute equally to the even and odd contributions to the flux divergences. However, it is clear from figure 4-31 that there are parts of the job which the western and varicose modes alone can not do which must be done by sinuous modes, even if the equilibrated state has sinuous and varicose modes transporting equal amounts of vorticity.

In summary, when the northern boundary is removed, there is a significant difference in the modes of variability which are possible. The sinuous modes (which break the antisymmetry of the double-gyre) are responsible for the inter-gyre flux of vorticity in the slip calculations via dissipative meandering. In no-slip calculations, they are responsible for a drastic increase in the efficiency of vorticity delivery to the frictional sublayer. With either boundary condition, they play a major role in controlling the circulation strength.

\section{Relevance of Multiple Steady-state Solutions and Equilibria}

As demonstrated by Cessi and Ierley (1990), there are multiple solutions to the steady-state, double-gyre calculation of two basic characters, exactly antisymmetric and asymmetric. The asymmetric solutions have a reduced circulation as the antisymmetric ones for the same forcing. The asymmetric solutions have a reduced circulation for two reasons. First, their shape is more conducive to the effects of friction. Second, the mean flow crosses the $y=1$ boundary between the wind stresses allowing a reduction of circulation input to each gyre as was in the case in figure 4- 
15. However, both sets of solutions are greatly in excess of the Sverdrup solution, as expected for a model without eddies (see section 3.8).

In previous chapters, it is made abundantly clear that the effects of eddies are critical in controlling the circulation strength, as they are the only mechanism of transport across mean streamlines in inviscid calculations. Figure 4-32 demonstrates that even in the strong circulation present before the breakdown of antisymmetry, the varicose eddy fluxes were already playing an important role in fluxing vorticity across mean streamlines similar to the one they play in the single-gyre calculations.

When eddies play a large role, it is tautological that the steady-state and timemean solutions be quite different. Steady-state solutions can only represent solutions where the friction is considered as a parameterization of the eddy fluxes. It is clear in the preceding chapters that friction and eddies behave in quite differently. The eddy fluxes are certainly important in this calculation, and thus it is unlikely that the steady-state solutions have much direct relevance.

On the other hand, multiple equilibria, as opposed to multiple steady-states, might play a significant role in these calculations. Note that in all of the double-gyre calculations here, the time-mean of the jet tilts slightly north on the western side of the basin. Symmetry demands that there be a similar equilibrium with the jet pointing slightly south. Clearly, the initial condition used has selected for the northward jet.

Given the nonlinearity of these calculations, it is likely that there are other equilibria than those presented here which could be reached using a different initial condition. It is highly likely, for instance, that there is an exactly antisymmetric equilibrium double-gyre calculation where each gyre is very similar to the single-gyre solution which is unstable to sinuous modes (although this is very difficult to reach numerically!). It is also possible, and perhaps even likely, that there are other equilibria which differ from those here, but are completely consistent with the governing equations.

Unfortunately, unlike in the steady-state calculations of multiple steady-states, it 
is extraordinarily difficult to find the bifurcations where these new equilibria can be reached. Likewise, trying different initial conditions to locate the basin of attraction of one or the other of the equilibria requires an exponential expansion of computational resources. Thus, only a few forays using different initial conditions were attempted, and all resulted in results similar to those above.

\subsection{Boundary-Enhanced Viscosity with a Second Gyre}

Despite the increased efficiency of the sinuous modes in transporting the wind's vorticity input to the frictional sublayer and in dissipative meandering when slip boundary conditions are present, there remains a role for boundary-enhanced friction with an added second gyre. Of course, the intended use of the boundary-enhanced viscosity as a parameterization to represent boundary-related processes remains appropriate when a second gyre is present; it remains unfair to ask a constant viscosity to represent a heterogeneous collection of physical phenomena.

Equilibrium is reached when a balance between the inflow and the outflow of vorticity is achieved, and the outflow of vorticity is affected by the amount of vorticity stored in the basin. Although the sinuous modes are more efficient at transporting vorticity to the frictional sublayer and require a much smaller circulation strength to do so, for a small enough viscosity even these modes cannot prevent takeover by inertia of the basin interior at all Reynolds numbers, because of the large time-mean vorticity gradients required to remove vorticity from the basin when the boundary viscosity is small.

Figure 4-37a demonstrates the eventual failure of constant viscosity even in the presence of an additional gyre. Figure 4-37a shows that an inertially-dominated solution can result even when sinuous modes of instability are present; however, inertial-domination is postponed until a much higher Reynolds number by the sinuous 

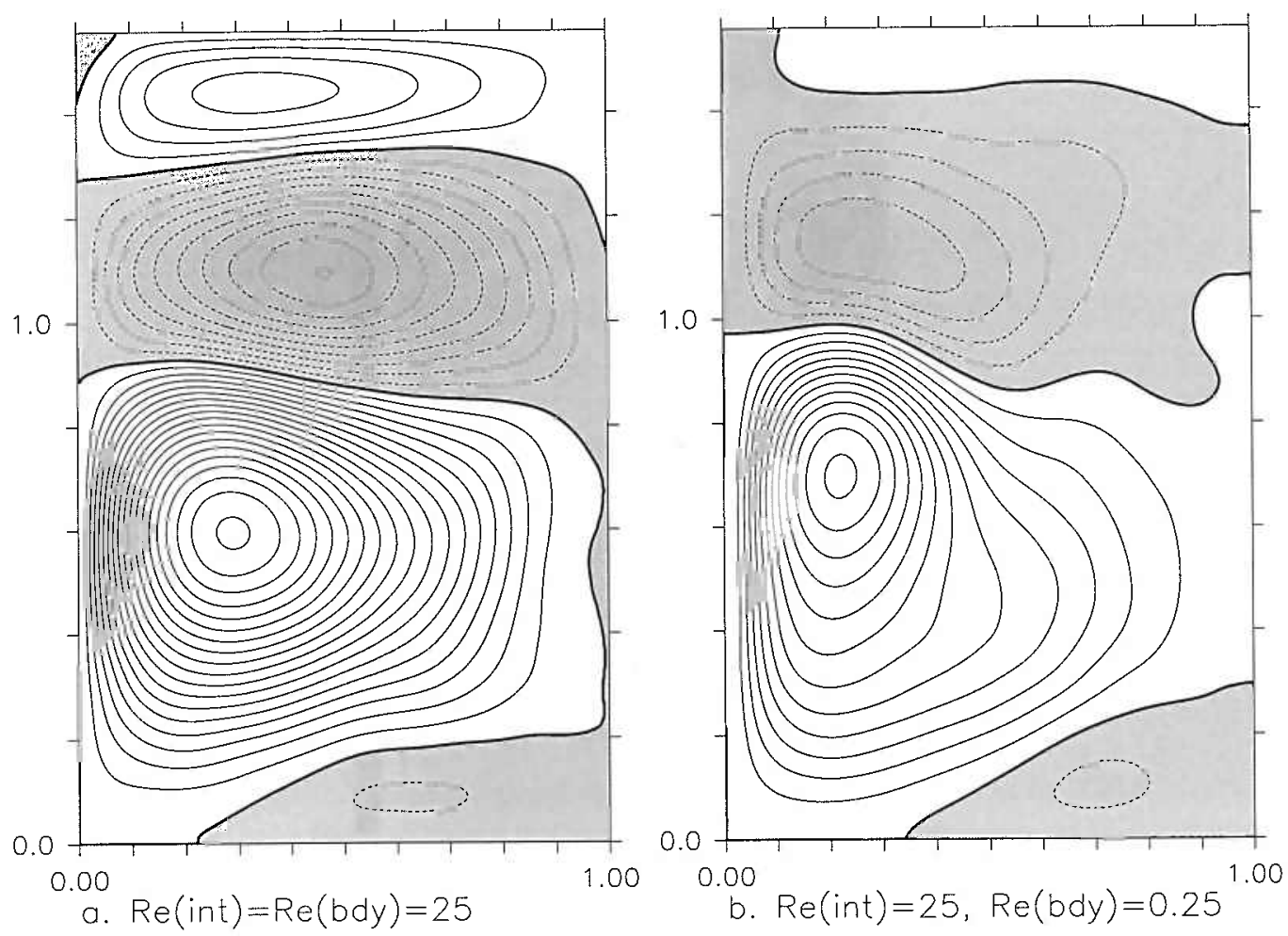

Figure 4-37: Time-mean streamlines of the (a) no-slip, two-gyre calculation with $\operatorname{Re}(\mathrm{bdy})=25, \operatorname{Re}(\mathrm{int})=25, \delta_{I}=0.06$ and (b) no-slip, two-gyre calculation with $\operatorname{Re}(\mathrm{bdy})=0.25, \operatorname{Re}(\mathrm{int})=25, \delta_{I}=0.06$. The contour interval is 0.1 .

modes.

However, the removal crisis in the inertially-dominated single-gyre featured a reversal in sign of the eddy flux divergence in the western frictional sublayer. In the inertially-dominated two-gyre calculation here, the reversal in sign is not present. Figure 4-38 shows that the eddy flux divergence of the inertially-dominated two-gyre calculation does not have this feature.

Nonetheless, figure 4-37b shows that when boundary-enhanced viscosity is used, the solution can be made more western-intensified even with $\operatorname{Re}($ int $)=25$, and even when the structure of the removal crisis is different. At this Re(int) the frictional 


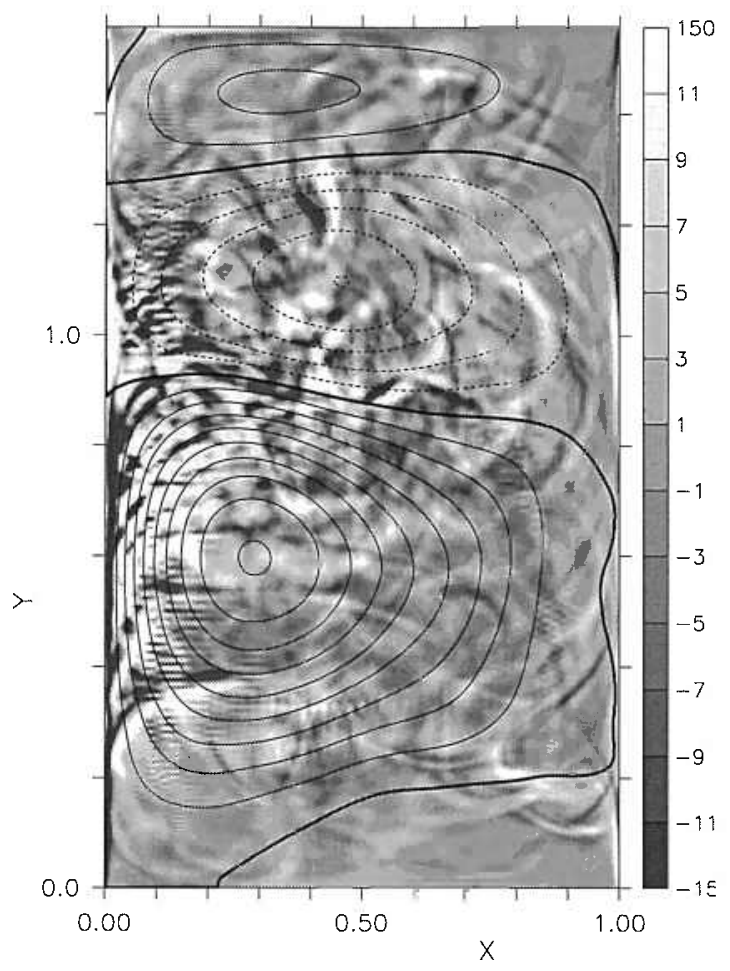

Figure 4-38: The time-mean streamfunction contours are superimposed on the eddy flux convergence shaded for the $\operatorname{Re}(\mathrm{bdy})=25, \operatorname{Re}($ int $)=25$, no-slip, two-gyre calculation. Lighter shading denotes convergences (which remove vorticity of the sign of the wind input); darker shading denotes divergences.

sublayer (and the region of boundary-enhanced viscosity) is 5 times narrower than the inertial boundary layer. Thus, the viscosity is $95 \%$ of the way to its interior value by 0.03 , so most of the fluid in the boundary current does not pass through the region of enhanced viscosity. Even so, the circulation strength is controlled by the boundary-enhanced viscosity, and is quite close in magnitude in the eastern part of the basin to the linear, non-inertial steady-state solution. Thus, aside from the counter-rotating regions, its eastern half is nearly in Sverdrup balance (as shown in 


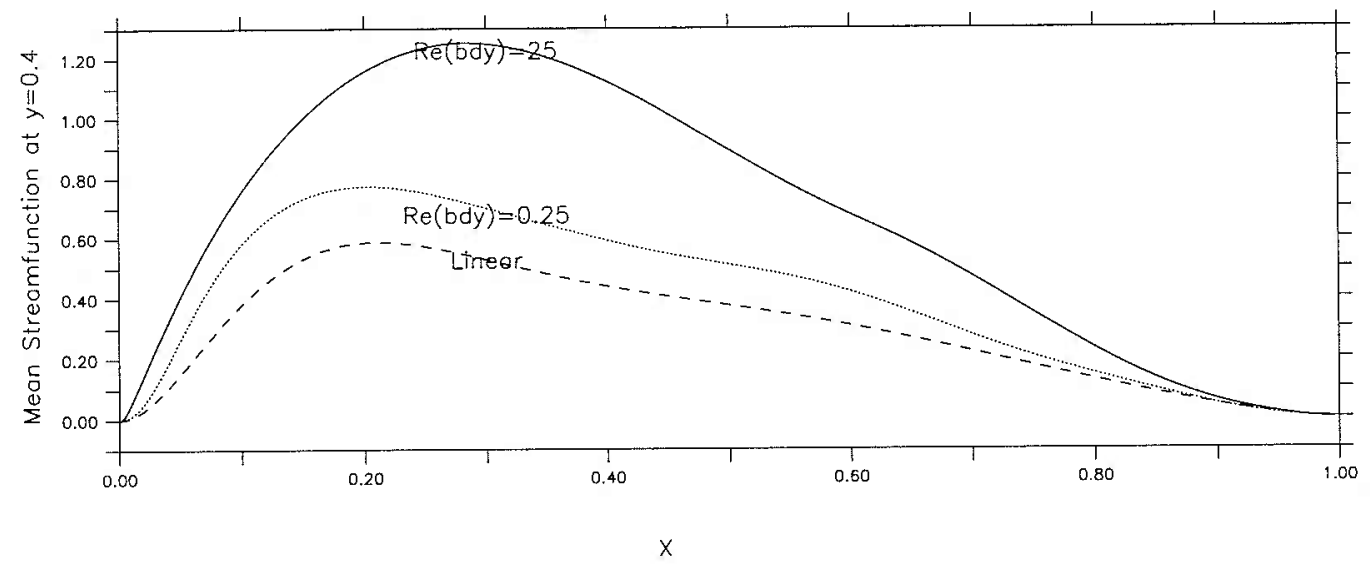

Figure 4-39: Time-mean streamlines of the (a) no-slip, two-gyre calculation with $\operatorname{Re}(\mathrm{bdy})=25, \operatorname{Re}(\mathrm{int})=25, \delta_{I}=0.06$ and (b) no-slip, two-gyre calculation with $\operatorname{Re}(\mathrm{bdy})=0.25, \operatorname{Re}(\mathrm{int})=25, \delta_{I}=0.06$. The contour interval is 0.1 .

figure 4-39). A rough approximation of the inter-gyre eddy flux ${ }^{7}$ estimates that only $10 \%$ of the vorticity input by the wind is cancelled by an inter-gyre flux of vorticity. Thus, the control of the circulation strength is still caused primarily by an eddy flux of vorticity to the frictional sublayer similar to that described in section 2.3 .2 , but in figure 4-37 the vorticity transport is aided by the sinuous eddies' efficient vorticity transport.

Rough estimation of the expected increase in computer power based on the current exponential rate of processor speed increases reveals that well-resolved calculations at this Reynolds number and $\delta_{I}=0.02$ should be routinely calculable by these methods on commonly available computers in less than 20 years. However, even then the viscosity would be many orders of magnitude larger than the molecular viscosity. The increasing resolution would allow the eddy viscosity to continue to be lowered in the basin interior, but until the boundaries' physics are explicitly resolved, the removal of vorticity there is needed. Thus, parameterizations such as enhancement

\footnotetext{
${ }^{7}$ It is rough due to the numerical difficulties of averaging at this Reynolds number.
} 
of the viscosity at the boundary will remain important.

There are many difficulties in making calculations at this Reynolds number. For figure 4-37, the inertial boundary layer width was increased relative to the basin dimension to ease the burden on resolution. This is equivalent to reducing the size of the basin $\left(\delta_{I}=0.06\right.$ in figure 4-37, elsewhere in the dissertation $\left.\delta_{I}=0.02\right)$. This increase was necessary so that the frictional scale would be able to be resolved. Unfortunately, this increase in the boundary layer width makes the streamlines of the inertially-dominated calculation and those of the western-intensified calculation appear similar. However, it is clear from figure 4-39 that one of these solutions is nearly in Sverdrup balance while the other is not. Even with this increase in the boundary layer widths, the resolution for these calculations is marginal when compared to the other calculations in this dissertation, with interior collocation points separated by approximately the Munk length. The spectral decay of the relative vorticity indicates that there are errors present in the calculation due to aliasing and truncation of a few percent.

Interestingly, both of the calculations shown in figure 4-37 possess intermittent periods where the kinetic energy grows by approximately a factor of 5 . This behavior, while fascinating, means that the averages which are presented in figure 4-37 are somewhat more approximate than those elsewhere in the dissertation.

Boundary-enhanced friction also plays a meaningful role in calculations with slip boundary conditions, even at moderate Re(int).

In figure 4-12, the boundary-enhanced viscosity reduced the inter-gyre vorticity flux. This reduction is apparently due to both the direct action of the friction, which now removes more of the vorticity with the same mean vorticity gradient, and to a lesser degree to a small eddy flux of vorticity to the frictional sublayer, an effect apparently absent in the constant viscosity case.

When boundary-enhanced viscosity is used in the two-gyre slip calculation, the eddy flux of vorticity to a frictional sublayer and the inter-gyre flux of vorticity are 

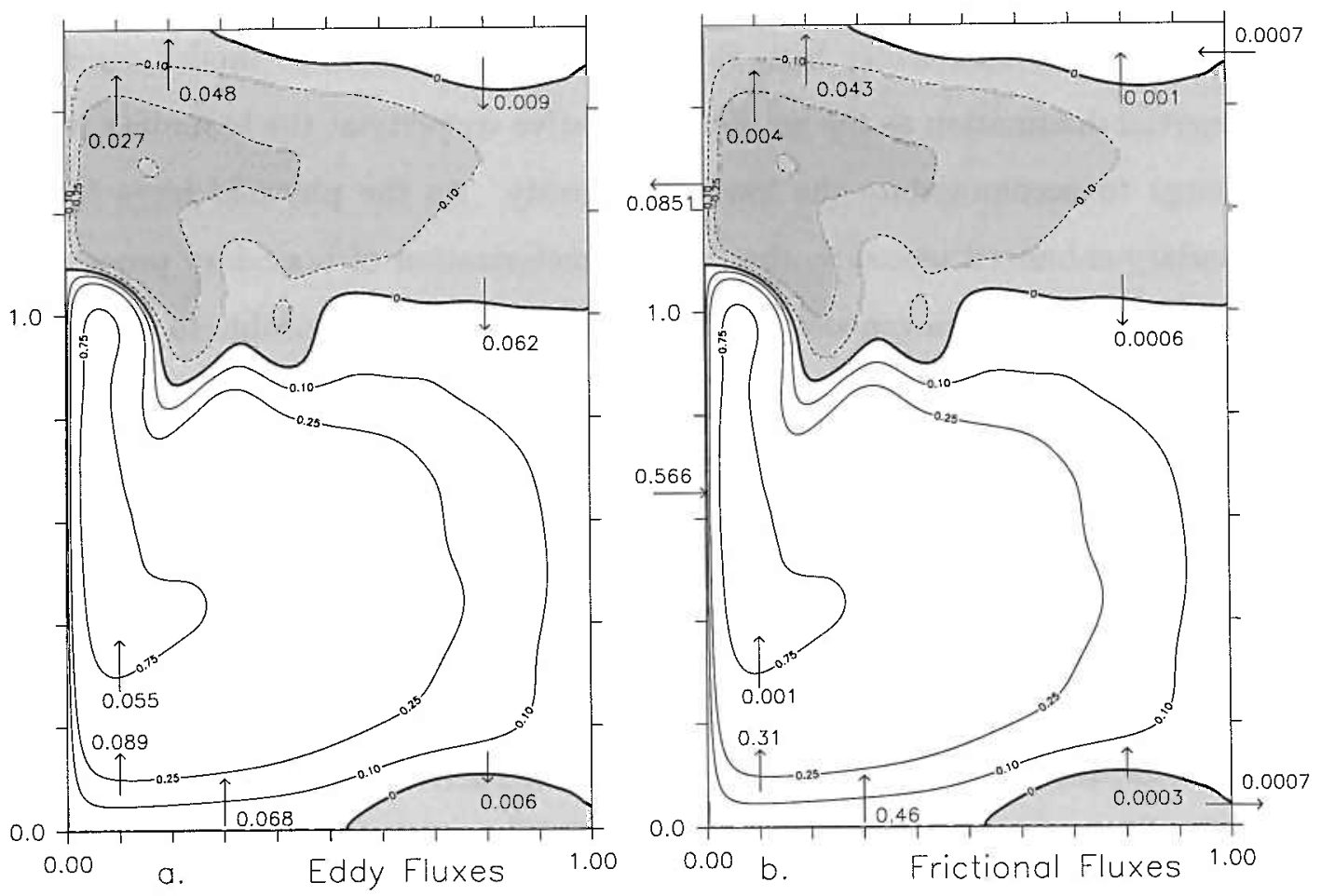

Figure 4-40: Time-mean eddy fluxes and frictional fluxes as in figure 4-5 except the calculation shown is the slip, two-gyre, $\operatorname{Re}(b d y)=0.25, \operatorname{Re}(i n t)=5$ calculation. Note that only selected streamlines are displayed.

both present. The use of boundary-enhanced viscosity in the slip calculations with multiple gyres allows for an eddy flux of vorticity to the boundary where there was none in the constant viscosity case, just as it did in the single-gyre case. However, as in figure $4-12$, the primary effect is that the inter-gyre flux of vorticity is decreased as the direct action of the enhanced viscosity on the mean flow viscosity increases.

Interestingly, a second effect of boundary-enhanced viscosity in the two-gyre calculations is that the inertial overshoot of the two-gyre slip calculations is dramatically reduced when enhanced viscosity is used. It is unclear whether at higher Re(int) this remains to be the case. 
To summarize, although the sinuous modes of variability greatly decrease the circulation strength by making the transport of vorticity to the frictional sublayer more efficient, at sufficiently high Reynolds number even these modes cannot prevent inertial domination as the gradient of relative vorticity at the boundary becomes very large to accommodate the lowered viscosity. As the physical basis for using a boundary-enhanced viscosity, that is, parameterization of boundary processes, remains true for any homogeneous model calculation, it seems sensible to use it in this case, just as it can be used in the single-gyre.

\subsection{Conclusion}

Adding a second gyre to the homogeneous model significantly changes the results. In the single-gyre, the frictional removal of vorticity through the boundary was inevitable. However, when the wind forcing is of different sign in different regions with the basin, not all of the vorticity need be removed from the basin. However, this exchange of vorticity is not guaranteed, and it depends critically on the details of the flow, especially the dynamics near the western boundary.

The choice of boundary conditions plays a surprisingly important role in calculations with multiple gyres. In the case of the double-gyre, slip calculation, an inter-gyre eddy flux of vorticity is the dominant mechanism for the removal of vorticity. On the other hand, the double-gyre, no-slip calculation has an inter-gyre vorticity flux which is utterly negligible in the statistically-steady equilibrium.

The difference between the calculations with different boundary conditions can be be understood with two observations. First, slip boundary conditions are unrealistically deficient in instabilities near the boundary. Thus, they are essentially unable to effect a significant eddy flux to a frictional sublayer. Second, the inter-gyre vorticity flux in the slip calculation occurs primarily by dissipative meandering. Once the jet is held in place by friction, as it is in the no-slip calculation, dissipative meandering 
is nearly impossible. Furthermore, unlike in slip calculations, the boundary current in a no-slip calculation contains relative vorticity of both signs, and therefore when it overshoots to the other basin its vorticity anomaly is canceled out to a large degree. Without this mechanism for inter-gyre eddy vorticity flux only the relatively weak mechanisms of transient exposure to opposing wind forcing and exchange of fluid parcels remains.

The inter-gyre eddy flux's role in slip calculations is further diminished by the results from the two-gyre, slip calculation. In that case, instead of two equally strong western boundary currents meeting at the jet separation, the subtropical boundary current dominates. This domination results the mean flow overshooting into the subpolar wind forcing region. As the Reynolds number increases, the subtropical gyre takes over more and more of the subpolar gyre. As it does so, the vorticity flux carried by the overshooting mean flow has the effect of exposing the subtropical gyre to less and less net circulation input while at the same time giving it a longer boundary layer to dispose of vorticity. The inter-gyre eddy flux remains the only mechanism for removing the vorticity input to the subpolar gyre as the subpolar gyre is cut off from the western boundary, but the inter-gyre eddy flux is not the dominant remover of vorticity from the entire basin in high Reynolds number calculations.

A profound difference between the single-gyre and the multiple-gyre calculations, which also explains the reduction in circulation strength in the no-slip calculations, is the introduction of sinuous modes with the removal of the northern boundary. These eddies are able to effect an efficient transport to the frictional sublayer which does not require a strong interior flow. This mechanism requires no inter-gyre eddy flux, agrees with the results of simulations of free jets, and is present to some degree in all of the calculations here, regardless of boundary condition. The eddy flux is so efficient, in fact, that the vorticity is dissipated at the same latitude where it is input, just as in the linear calculations. Sinuous modes are also responsible for the dissipative meandering in slip calculations. 
The sinuous modes are able to increase the efficiency of the vorticity transport to the frictional sublayer in the no-slip calculations. That is, they are very active in producing eddies which can transport vorticity from the region where the recirculation gyre would form, yet unlike the modes of instability present in the single-gyre calculation, they do not require a strong interior circulation to make them energetic enough to perform this flux.

Thus, the circulation strength is greatly decreased when the sinuous modes are present. However, at sufficiently high Reynolds number, even these modes cannot succeed in controlling the circulation strength with a constant viscosity, for like the eddies in the single-gyre, they cannot ultimately remove vorticity from the basin. At the largest Reynolds numbers performed in this dissertation, inertial-domination occurs even in a two-gyre calculation, as the vorticity gradient at the boundary (required to remove the vorticity with small viscosity) becomes too large. The use of boundary-enhanced viscosity is effective in this case in reducing the circulation strength by reducing this gradient, even though the region of the frictional sublayer is very narrow. 


\section{Chapter 5}

\section{Conclusion}

\subsection{Wind-Driven Circulation Control by Boundary- Enhanced Viscosity}

In chapter 2, calculations of the homogeneous model are presented which indicate that the circulation strength of the wind-driven ocean is easily controlled in the single-gyre model by adding a region of enhanced viscosity near the boundary. The enhanced viscosity is a parameterization of all of the processes that are capable of removing vorticity from the basin. This region is needed in the homogeneous model because the simplicity of the model and its topography do not allow for the boundaryrelated processes to occur, regardless of the resolution of the model. Therefore, in this model, the eddy viscosity is interpreted as a parameterization of boundary process and eddies separately, the dilemma of inertial-domination of the single-gyre at moderate Reynolds number is resolved, at least for the parameter range achieved here.

This region of increased viscosity used to parameterize boundary processes here is narrow; it decays with the frictional sublayer scale. In many of the calculations here, its decay scale is less than half the inertial boundary layer width, and in the highest Reynolds number calculation, it is effective in decreasing the circulation strength 
(with the help of the sinuous modes) even when it is more than five times smaller than the inertial boundary layer width. Thus, in a $100 \mathrm{~km}$ wide boundary layer, the direct effects of the boundary processes may be effective were they contained in only the shoreward $20 \mathrm{~km}$ of the current. The results of this dissertation give no reason to expect that the size of this region of interaction with the boundary could become too small. So long as the boundary processes easily remove vorticity, it is likely that an even smaller region would suffice.

A reasonable question to ask is whether this process can reasonably be continued until the molecular viscosity is reached and the Reynolds number is on the order of millions or billions. In this case, the frictional sublayer defined as it is here would be only a thousandth or less of the width of the inertial boundary current. Yet, all of the vorticity input would still need to be removed there, according to the ideas presented here. Obviously, this model is not intended to be taken to this absurd limit. The legitimacy of not only the quasi-geostrophic limit, but even the shallow-water limit will break down long before this occurs. Important effects of fully three-dimensional turbulent fluid dynamics would need to be considered before considering such a small region for frictional removal.

The results of this dissertation are intended, however, to show that it is not necessary for all of the mean flow to pass through the frictional region or the region where vorticity is otherwise ultimately removed. This region can be contained in a region near the boundary which is narrower than the inertial boundary layer and the eddies will communicate the vorticity input within these interior streamlines to the boundary for removal. Although the parameterization used here choses a region of frictional removal which becomes smaller and smaller with increasing Reynolds number, this is a function of the parameterization and is not necessarily a reflection of reality. If, for example, an interaction with bottom topography were responsible for the removal of vorticity, one would expect this region to remain fixed in size over the region of sloping or rough topography as the Reynolds number changed. The results 
of this dissertation only indicate that whatever type of vorticity removal exists, it need not be in an area larger than the inertial boundary layer width to participate in the control of the overall circulation, and not all of the mean flow need pass through its region of vorticity removal.

There is also no problem in the keeping the eddy fluxes large enough, because almost immediately after the inertial boundary width is wider than the region of ultimate removal, almost all of the wind's vorticity input is carried across the interior mean streamlines by the eddies. The amount of this flux does not grow with increasing Reynolds number, so there is no additional burden on the eddy fluxes. That is, regardless of how much of the eddy flux is parameterized and how much is resolved, the total flux is constant (although it may change for a particular streamline). Since we began with parameterized eddy fluxes of the same order of magnitude as found by LaCasce and Bower (2000) (that is, a Reynolds number of order 1), maintaining this magnitude of eddy flux as more of the eddies are resolved is no problem.

One might think that perhaps this model requires too much eddy kinetic energy to carry the vorticity flux. However, Wunsch (2002) analyzes the ratio of eddy kinetic energy to mean kinetic energy from TOPEX/POSEIDON satellite altimetry observations and finds that there is 25 to 100 times more kinetic energy in the eddies than in the mean flow in the region of the western boundary currents. Although the model's physics and the type of eddies present differs significantly from the real ocean, the models here have not exhausted this limit; eddy kinetic energies exceed the mean flow kinetic energy by only a factor of 3 or 4 in the models (see figure $2-4$ ). This ratio does grow with increasing $\mathrm{Re}(\mathrm{int})$, but there is room for a two order of magnitude increase and a more sophisticated model could have a very different parametric change in this ratio.

If the region of ultimate removal is small, however, there could be a fair burden on this region as the torques there must balance the wind input over the whole ocean. It seems reasonable to expect this theory to extend perhaps a few orders of magnitude 
between the inertial boundary layer width and the width of the region of boundary processes parameterized here as frictional removal. But, it is important to remember that the proposed shrinking of this region is part of the parameterization used here, not part of the oceanic process. If, for example, an interaction with bottom slope were responsible for the removal of vorticity as proposed by Hughes and De Cuevas (2001), the size of the removal region would be probably less than an order of magnitude smaller than the inertial boundary layer. An increase in the bottom torques by less than an order of magnitude is entirely reasonable. If smaller regions were responsible, one would expect that the higher velocities (especially higher bottom velocities with significant topographic interaction) typical of continental shelf dynamics to have more than enough room to dispose of this vorticity. Furthermore, as the region of removal get shallower, less momentum is required to change the absolute vorticity. Thus, it seems that there is quite a bit of room for the removal of the vorticity, so long as the region of ultimate removal is within a few orders of magnitude of the inertial boundary current width.

The control of the circulation can be described in terms of the vorticity fluxes which transport and remove the wind input of vorticity. In a western-intensified single-gyre solution, the wind's vorticity generates a $\beta$-flux of vorticity carried by the mean flow toward the western boundary. The mean flow enters a boundary current (in a manner consistent with the dynamics of Charney (1955) and Ierley and Ruehr (1986)) as the vorticity flux is transformed to a mean advective flux. When the steady-state boundary current solution is no longer exists or becomes unstable, the boundary current forms instabilities. The eddies formed by these instabilities transport the vorticity across the mean streamlines to a frictional sublayer where it is removed from the basin by a frictional flux.

In calculations which are very viscous, so that the frictional boundary layer width is significantly larger than the inertial boundary layer width, the solutions tend to be steady. However, as pointed out by Ierley and Ruehr (1986), these steady solutions 
soon disappear. For a small range of viscosities beyond this point, unsteady westernintensified equilibria are found. At still smaller viscosities, the effects of inertia are no longer contained in a region near the boundary, and the solution becomes inertiallydominated, as situation quite unlike the real ocean.

However, since the viscosity is in part a representation of the effects of eddies, it should be possible to lower the viscosity and resolve more and more eddies. But, because these eddies cannot remove vorticity from the basin, a removal crisis occurs.

If the viscosity near the boundary is insufficient for the friction to remove the vorticity input, the relative vorticity builds in the basin, increasing the gradients near the boundary until the frictional flux is sufficient to achieve equilibrium. These large gradients in the vorticity have a profound effect on the character of the solution, however.

In the single-gyre calculations, these large gradients somehow cause the eddy fluxes divergence to change sign within the western frictional boundary layer. This change in sign is accompanied by a change in sign of the frictional flux through the basin boundary nearby. Thus, instead of removing the negative vorticity input by the wind, in this region the frictional flux provides more negative vorticity to the basin. This implies that the frictional fluxes elsewhere must increase their rate of removal of the wind's vorticity input, and in doing so further increase the vorticity gradients near the boundary changing the character of the solution further.

When the eddy flux divergence changes sign, the equilibrium is inertially-dominated, and the eddy fluxes are no more efficient at delivering vorticity to the frictional sublayer than the mean fluxes. Thus, the advantage of eddy fluxes over the mean fluxes of vorticity, that they can transport vorticity across the mean streamlines, is lost.

When the vorticity is allowed to build in the recirculation gyre, it increases in strength until it reaches the eastern boundary where it is able to draw in additional eddies from the eastern frictional sublayer. It is the eddies from the eastern frictional sublayer which eventually allows the inertially-dominated solutions to equilibrate. 
Whether the enlargement of the recirculation gyre is a response to the reversal of sign of the eddy flux divergence in the western boundary layer or a cause of it is unclear. However, the two phenomena are intrinsically linked and characteristic of the inertially-dominated single-gyre calculations. In fact, a qualitative change in the temporal and parametric behavior of the single-gyre occurs as soon as the recirculation gyre reaches the eastern boundary, and it never does so without a reversal of sign of the eddy flux divergence in the western frictional sublayer.

When a second gyre is added, new modes of instability are present along the jet. In particular, the sinuous modes of instability of the jet have rapid growth rates and are effective at mixing across mean streamlines to either side of the jet. If care is taken to prevent these modes from occurring the dynamics of the double-gyre are very much like those of the single-gyre. Once these modes are present, however, the dynamics are quite different.

In the calculations of the two-gyre and double-gyre models with no-slip boundary conditions, despite the promise of internal cancellation by inter-gyre flux of vorticity almost none occurs. This is likely due to the fact that the jet dividing the gyres forms a barrier to eddy transport of both mass and vorticity, and that the no-slip boundary condition prevents dissipative meandering of the jet.

Despite the lack of inter-gyre vorticity flux, the circulation strength is greatly reduced in the two-gyre and double-gyre no-slip models when compared to the singlegyre model. The only theory presented here which is able to explain this decrease in circulation strength is that the presence of sinuous modes make the transfer of vorticity input by the wind to the frictional boundary layer very efficient. That is, the sinuous modes and the western boundary modes of variability are able to work together to flux vorticity across mean streamlines to the frictional sublayer where it is removed without requiring a strong interior circulation. This differs from the single-gyre case where the eddies are not very strong until the circulation strength is quite large. The efficient transport of vorticity by the eddies prevents vorticity 
from pooling in the basin interior, so that the recirculation gyre is almost completely absent at Reynolds numbers where the single-gyre would be inertially-dominated.

However, the sinuous modes cannot prevent inertial-domination indefinitely, for they cannot remove vorticity from the basin. Only the frictional fluxes can do that in this model, and to do so they require larger and larger vorticity gradients at the boundary for smaller and smaller viscosity. Eventually, these gradients cause inertial effects which are powerful enough to overwhelm even the efficient sinuous modes of instability, and the equilibrium becomes inertially-dominated. At this point, the mechanism of boundary-enhanced viscosity returns to importance, and it is demonstrated in chapter 4 that it is also effective in reducing the circulation strength of the two-gyre calculations.

Interestingly, the two-gyre wind forcing was chosen so that there would be a net vorticity input to the basin, but in the case of the no-slip calculations, even at the highest Reynolds numbers, the gyres do not communicate significant amounts of vorticity. Thus, in this case, the important feature of the two-gyre calculation is that it is useful in understanding the broken symmetries required for the sinuous modes.

This result in the no-slip calculations is surprisingly different from previous results where the inter-gyre vorticity flux was the dominant source of vorticity removal. Calculations of the slip, double-gyre model performed here and in Lozier and Riser (1990) indicate that the inter-gyre vorticity flux in that case is primarily carried by dissipative meandering. This process relies critically on the meandering of the jet very near the western boundary layer, and the bias in sign of the relative vorticity contained in the boundary current. Thus, the effects of dissipation near the boundary are again critical, even in inter-gyre eddy flux, a process which could in principle be inviscid. Again, the sinuous modes of instability are important, because they are required for dissipative meandering to take place. Dissipative meandering does not occur in the no-slip calculations because the jet separation is not able to move along the boundary and the vorticity within the boundary current can be of either sign. 
In the case of the slip, two-gyre model, the inter-gyre eddy flux is no longer the dominant mechanism for vorticity removal. Instead, the subtropical western boundary current overshoots the location where the wind forcing changes sign. As the Reynolds number increases, this overshoot increases forming a recirculation gyre at the northern boundary. More and more of the subpolar gyre is replaced by this recirculation. Thus, most of the wind forcing is applied to the subtropical gyre's mean flow. The removal of vorticity from this gyre is benefited by the elongation of its western boundary current and by an eddy flux to the frictional sublayer. Only the wind forcing to the much smaller subpolar gyre is canceled by an inter-gyre eddy flux. Thus, with slip boundary conditions, it is very important that there is a net vorticity input to the basin in the two-gyre calculations, because it is the gyre with the larger vorticity input which enlarges to fill the entire basin.

Thus, in all of the models here, the effects of friction near the boundary plays an important role in accepting the eddy fluxes from the interior or in causing the dissipation for the dissipative meandering. So, not only the transport of vorticity but also the removal of vorticity from the wind-driven gyre is an integral part of controlling the circulation strength.

\subsection{Method of Westward Intensification at High Reynolds Number}

The mechanisms which select for a western rather than an eastern boundary layer are the same mechanisms which allow for a flux across mean streamlines. For this reason, the flux across the streamlines occurs primarily in the west. First, during spin-up and afterward in nature of the eddies, westward propagation is selected. Second, the removal of vorticity from the gyre is selected to occur primarily on the western boundary by a frictional sublayer.

However, what causes Stommel's crowding of streamlines is the westward $\beta$-flux 
of the wind's vorticity. The subsequent intensification is caused by a mechanism very similar to the theory of Charney (1955) and Ierley and Ruehr (1986), forming a visco-inertial boundary layer.

The solution to the problem of rejoining the models of Charney (1955) and Ierley and Ruehr (1986) is solved by the turbulent boundary layer, a boundary layer quite similar to found by Edwards and Pedlosky (1998), although the context is quite different here. This boundary layer is a turbulent frictional boundary layer where the change in absolute vorticity required to rejoin the interior flow is carried out by an eddy flux convergence. The eddy fluxes created in the process end in the frictional sublayer where the are removed from the basin.

\subsection{Behaviors of the Relatively Inviscid Single-Gyre Model}

Once equilibria which are relatively inviscid in the interior and relatively westernintensified are made possible by boundary-enhanced viscosity, a number of phenomena are observed with potentially interesting consequences in the real ocean.

In some pairs of single-gyre calculations with different $\operatorname{Re}(i n t)$ and $\operatorname{Re}(b d y)$, the time-mean fields and the eddy flux divergences were surprisingly similar. However, the eddy fields were quite different. Therefore, the large changes in the eddies resulted in only small changes in the divergences, and the change in the viscosity (together with the action of the other terms) compensated, or parameterized the changes in the effects of eddies on the mean flow.

When the boundary current instabilities are in the appropriate frequency range, they excite the basin modes of variability. While the instabilities which excite them appear to be contained near the western boundary, the basin modes are often the dominant EOF of the streamfunction perturbation, and they can become strong enough to affect the mean flow by nonlinear interaction. 
Studying the EOFs revealed not only the basin modes, but other modes of variability in the western boundary current which had fairly sharp frequency peaks. However, as Re(int) became large, so did the number of instabilities and the amount of interaction. Rather than a few frequencies associated with the primary instabilities and responses to nonlinear interactions at the sums and differences of their frequencies, the frequency spectrum became quite broad. Thus, the variability occurred on many time scales, including very slow, climatic time scales. These calculations undergo dramatic changes

One primary effect of the presence of basin modes is the appearance of a counterrotating gyre at the opposite corner of the basin from the recirculation gyre. This counter-rotating region has a vorticity balance which is primarily between the eddy flux divergence and the wind input. At higher Reynolds number, these regions often fill much of the basin which might have been in Sverdrup balance, thereby changing the local balance of $\beta$-term to forcing there to a nonlocal eddy to wind forcing balance.

The flux convergence patterns causing the counter-rotating regions appear to be consistent with the effects of the basin modes. Furthermore, when it is arranged so that there are regions without wind forcing where the counter-rotating gyres are expected to appear, they do appear, although their fundamental balance of terms in the vorticity equation is quite different. In those regions, they are primarily a balance between the eddy flux convergence and the $\beta$-flux divergence. In this way, and in their configuration, they strongly resemble the basin mode-mean flow interactions analyzed by Pedlosky (1965b).

The energetics of the solutions reveal much the same story as the vorticity does. As the interior Reynolds number increases, the percentage of removal of energy from the mean flow by the direct action of friction decreases and the percentage of removal by the production of turbulent kinetic energy increases. The boundary-enhanced viscosity is able to reduce the energy of the solution in two ways. First, the direct removal of energy by friction is contained primarily in the frictional sublayer. Second, 
unlike in the input of vorticity which is always fixed, control of the circulation strength decreases the wind's energy input.

\subsubsection{Higher Reynolds Number?}

The highest Reynolds number used here is only 25, which means that the frictional sublayer scale is only 5 times smaller than the inertial boundary current. Likewise, there is only a small separation between the scale of the eddies and the viscous dissipation scale. It would have been nice to go to somewhat higher Reynolds numbers to have a more fully-developed inertial range, but computational constraints were prohibitive.

The reasons why the calculations have such a limited Reynolds number range is twofold. First, the whole basin was resolved. With an inertial boundary layer width of 0.02 , this means that a factor of 50 was sacrificed in resolution in order to have a relatively narrow boundary current. Secondly, because the frictional terms are the critical variable parameter differentiating the different calculations, it was necessary to guarantee the accuracy of the frictional terms. This requires accurate evaluation of the fourth derivative of the streamfunction without dissipative numerical errors. Many other calculations are not so strongly focused on the precise form of the frictional terms, so these terms are evaluated to only moderate accuracy or numerical dissipation is allowed. I chose to take great care to properly evaluate the frictional terms, which limited the maximum Reynolds number as well.

It is worth mentioning here what may have been some of the results were I able to resolve more of the inertial range.

One of the peculiar aspects of having only large eddies to do the job of fluxing the vorticity to the boundary layer is that they are almost all of the scale of the boundary current (or greater if the basin modes are included). Thus, when one of these eddies is in the region of the boundary current, the boundary current may be halted, strongly redirected, or even reversed depending on the strength of the eddies. 
This can be seen in figure 2-5. In the real ocean, eddy events of this magnitude are extremely rare or nonexistent. Presumably, if the flux across mean streamlines could be carried by smaller eddies, fewer interruptions might result.

Also, it is demonstrated in Chapter 3 that as Re(int) becomes large, the temporal variability becomes less peaked and more broadband. This is an indication of the increasing variety of nonlinear interactions with increasingly divers frequencies. Presumably, were the inertial range much larger, the spectrum would be much flatter (with the possible exception of the analogues of the basin modes). That is, there would be a more turbulent regime, instead of a weakly nonlinear or moderately nonlinear perturbation to the system.

However, I believe that, as far as the control of the circulation strength is concerned, most of the effects of resolving the eddy field are already present in these calculations. I believe that there are likely to be more profound changes with the introduction of vertical structure than with the extension of the inertial range. Thus, if more computer power is to be spent on this problem, baroclinic effects should be included before continuing to higher horizontal resolution.

\subsection{Future Directions of Research}

\subsubsection{Toward a Realistic Model of Vorticity Removal at the Boundary}

This dissertation does not address specifically what processes are parameterized by the boundary-enhanced friction, nor how they operate. Although it is now clear that such processes would be of use in controlling the circulation strength, they are not identified. Also, it is likely that some of the details of the results found here are dependent on the form of the parameterization used. In this section I propose a few mechanisms and cite the work of others which I think might be promising in rectifying this situation. 
However, it is important to point out that the main thrust of this research should hold regardless of the specific mechanism for vorticity removal. That is, the circulation strength of the entire basin can be controlled even when the inertial boundary current is wider than the region of vorticity removal. The innermost mean streamlines need not pass through the region of vorticity removal from the basin, as an eddy flux will transport the vorticity input within these streamlines across the mean flow toward the boundary if there is a mechanism for removal of vorticity located there.

This result becomes even more important when considering a moving layer of wind-driven fluid near the ocean surface which is insulated from the deeper fluid and the ocean bottom by stratification. This equivalent barotropic layer will have dynamics quite similar to those of the barotropic model studied here, and the removal of vorticity input must also be effected by lateral fluxes in an equivalent barotropic model. Only when the moving layer enters a region where the bottom topography rises to meet it will its dynamics be affected directly by the bottom topography. When the inertial boundary layer width is wider than the region of interaction with the topography, then the mechanism described herein of an eddy flux to the region of vorticity removal will be in effect.

\subsubsection{Topographic Interaction}

Hughes and De Cuevas (2001) have proposed an interesting mechanism for the removal of vorticity from the wind-driven gyre by bottom topography. They are able to show that if the bottom topography is correlated with the bottom pressures, then much as the pressure correlation to topography in lee waves implies a form drag, the bottom pressure on the ocean circulation can produce a form torque. They argue that so long as the frictional sublayer is narrower than the region of topographic interaction, this effect will be the dominant method of removal of the wind's vorticity input.

However, in their paper, they neglect the role of the inertial boundary layer. They argue that the inertial terms are negligible throughout the ocean (and the use a coarse 
resolution eddy-permitting model to 'prove' it!), and consider the boundary current width of importance to be the frictional ones. I propose that they should not only be concerned with whether the frictional layer is wider than the region of sloping topography, but whether the inertial layer is wider. As I have made clear here, the high Reynolds numbers that exist in the ocean make it clear that the frictional boundary layer should be quite narrow, but the inertial boundary layer is another matter entirely. A Charney boundary current will be $200 \mathrm{~km}$ wide in a $10000 \mathrm{~km}$ basin with a velocity scale of $1.6 \mathrm{~m} / \mathrm{s}$ (with $\beta=4 \cdot 10^{-11}(\mathrm{~ms})^{-1}$ ). Looking at the Maps (1988), it is easy to locate regions of the continental shelf east of North America and Asia where the depth changes from 500 meters to 3000 meters or more in less than $50 \mathrm{~km}$ and then changes slowly afterward. Thus, it is quite possible that the inertial boundary current could be wider than the region of sloping bottom topography, especially if the moving layer of fluid does not extend below a few thousands meters.

Hughes and De Cuevas (2001) use model results from an 1/4 degree calculation to show that their theory is effective in that a balance exists between the wind forcing and the bottom drag. I agree that such a result may be ultimately responsible for the removal of vorticity from the basin, but I believe that a significant step along the path from wind input to ultimate removal may be played by eddy fluxes. As the resolution required here to moderately resolve the effects of eddies in the boundary layer was better than and equivalent of 1/100 degree, I believe that their model was too coarse to accurately diagnose or rule out the role of eddies. A combination between the vorticity removal by form drag proposed by Hughes and De Cuevas (2001) for the outermost portion of the boundary current and an eddy flux of vorticity across the boundary current as described in chapter 3 is a very effective and relatively inviscid model of the vorticity removal from the ocean.

Topographic interaction with rough coastlines and shelves is also likely to produce eddies near the boundary, aiding in an eddy flux across the boundary current. Hallberg and Rhines (2000) show that the interaction of isopycnals with bottom slope can 
produce plumes of eddy activity which extend far into the interior even with steady forcing. These plumes separate in the interior to form potential vorticity anomalies. It would be very interesting to revisit this sort of calculation in light of the role of the boundary in production of eddies and vorticity here. Becker and Salmon (1997) show that the production of eddies is significantly different at a sloping boundary even in the case of single-layer calculations. They show that in situations where no eddies would be produced with a flat bottom, they are produced with over a sloping bottom. Certainly, the dearth of eddy activity formed near the boundary with slip boundary conditions (section 3.8 ) is likely to be artificial.

\subsubsection{Density Layer Outcropping}

With approximations to the reduced gravity rotating shallow water equations, Parsons (1969) was able to find a non-inertial solution to the wind-driven circulation with fully variable depth in a single layer. This model was extended by Veronis (1973), Huang and Flierl (1987) and Huang (1987) to include the subpolar gyre and multiple layers. Although all of these models have no advection of relative vorticity, in light of this research they present an interesting possible source of vorticity.

A shallow-water model which allows outcropping has a significantly different basinwide vorticity budget than the corresponding fixed-depth quasigeostrophic model. Two important differences are 1) the importance of the inter-facial friction terms in the vorticity budget is increased dramatically as the layer depth decreases, and 2) boundary layer separation (and outcropping) reduces the input due to wind stress. Although some work has been done on boundary layer separation including outcropping and even the vorticity dynamics of outcropping using isopycnal models (Chassignet and Bleck (1995), Chassignet et al. (1995), and Chassignet (1995) for example), the impact of these outcropping regions on the basin-wide vorticity budget in relatively inertial calculations has not.

Models with fixed depth such as the homogeneous model, or even the quasi- 
geostrophic model, miss the potentially important source of absolute vorticity at an outcropping boundary layer. In those models the input of vorticity at the surface is always controlled by the wind. In the presence of an outcropping boundary layer, however, the vorticity budget could be frictional at the ocean's surface. There are two reasons that this outcropping region might play an important role.

1) The interfacial friction flux cannot remove vorticity through the sidewalls in a no-slip calculation, but it can remove vorticity at an outcropping boundary where it can transfer the vorticity to the next layer.

2) Because the fluid layer is very thin at the outcropping and thus possesses little mass, relatively small forces are needed to change the layer's velocity. Although body forces will decrease along with the layer's mass and the layer thickness, surface forces such as the wind and interfacial friction will not decrease. Thus, a small imbalance in the surface forces may cause a relatively large change in the velocity when the layer is thin. The absolute vorticity budget does not consider the thickness of the fluid layer, just its velocity. Thus, where the layer is thin, relatively weak surface forces will have a larger effect on the absolute vorticity budget than where the layer is thick. Thus, even if the direct effect of interfacial drag is deemed negligible in most of the ocean where the layer is thick, interfacial drag could be quite important when the layer is outcropping and thin.

If a numerical model related to Parsons (1969) were created, it might reveal that there is a major component of the absolute vorticity budget to be found at the outcropping boundary layer. Chassignet (1995) begins an investigation into the vorticity dynamics of outcropping boundary layers, but because he doesn't use interfacial friction this potentially important sink of vorticity is not available.

Even if the vorticity supply is not significantly changed by the outcropping boundary layer, the outcropping clearly represents a new method of adjustment to wind stress as only the surface layer is exposed. According to the model of Parsons (1969), as the wind forcing increases the outcrop moves so that less and less of the surface 
layer is exposed. In this way, as the wind increases, its forcing is applied to a greater and greater depth of the ocean which reduces the forcing per unit mass.

Also, as mentioned above, the interaction with topography is a poorly understood and important part of the circulation. One of the major issues is that equivalent barotropic flows are effectively insulated from the effects of bottom topography. Where an outcropping boundary layer separates from the coastline can therefore control how much interaction with the topography occurs, so there is yet another feedback between outcropping and circulation strength.

\subsubsection{Other Processes}

If a quadratic bottom drag is more appropriate than Stommel's linear bottom drag, then the removal of vorticity at the boundary could be enhanced. Obviously the quadratic bottom drag would be larger where the bottom velocities are large, which occurs primarily near the boundaries.

McWilliams et al. (2001) have studied the limits of time integrability of the nonlinear mainly balanced dynamics, or slow manifold dynamics. They hypothesize that these breakdowns in the time integrability represent indications of the fluid-dynamical transitions which lead to the breakdown of the large-scale flow into smaller-scale flows. It seems that these ideas could be implemented as a spatially-dependent viscositylike operator and then examined as the homogeneous model was here. Whether the breakdowns of the slow manifold amounts to a boundary-enhanced viscosity would be a very interesting connection to the work here. The large gradients and the strong nonlinear interactions near the boundaries are obvious places to look for a breakdown in the balanced equations, so a boundary-enhanced removal of vorticity is a plausible result. 


\subsubsection{Sinuous Modes}

I was completely unprepared for the results concerning the sinuous modes in chapter 4, and had I known about these puzzling effects before beginning the dissertation, they may have been my main focus. These modes' profound effect on the circulation strength deserves much more attention. A significant amount of analytic studies with which I am unfamiliar would certainly have enlightened my discussion of these modes. Certainly, the question remains as to where these modes act to transport vorticity across the mean streamlines and precisely how they do it. These eddies are very active right on the region separating the gyres, why don't they transport more vorticity across the boundary in the no-slip calculation? The ratio of fluxes similar to a Nusselt number used here demonstrates that these eddies are more effective at mixing vorticity across mean streamlines than the western boundary variability, but why? The last chapter of a dissertation is usually an opening for many new ideas, and that is certainly true here.

The existence of the sinuous modes prevents the onset of inertial domination quite effectively in the multiple gyre calculations. However, it seems that the inertial domination eventually does occur. Because the single-gyre reaches an inertially-dominated state at a much lower Reynolds number than a multiple-gyre calculation, and because there is little or no inter-gyre flux to contend with in the no-slip calculation anyway, the single-gyre model is an excellent model to study the dynamics of inertial domination with less computational expense and complication. This is true for the time being given the substantial computational demands of getting inertially-dominated multiple gyre calculations like the one in figure 4-37a. Studies involving inertial domination including baroclinic effects and effects of topography, for example, would be significantly easier in the single-gyre. 


\subsubsection{The Three-Dimensional Problem}

\section{Effects of Depth Variation}

As mentioned above, allowing for layer outcropping in this model could introduce a new source of vorticity and change the interaction with the bottom topography or allow a layer to avoid wind forcing. However, simpler effects of depth variation are possibly more important in the real ocean.

Using a reduced-gravity model for calculations similar to those found here, the speed of wave propagation and the frequencies of the basin modes would change. This would allow for tuning toward or away from the frequencies of the instabilities. Changing the wave speed in a multiple-gyre calculation would also affect the eddies' ability to travel against or across the jet. Also, as the radius of deformation changes the typical scale of eddies formed, presumably this would have an important impact on their ability to interact with the frictional sublayer.

Cessi and Primeau (2001), Primeau (2002), and Lacasce and Pedlosky (2002) have all found profound differences in the behavior of baroclinic basin modes and Rossby waves when compared to the behavior of barotropic waves. The dissipative selection of low-frequency modes proposed by Cessi and Primeau (2001), and the resonances of low-frequency modes proposed by Primeau (2002) would be very interesting to study in the relatively inviscid limits explored here. These modes are quite likely to be unstable in this model, and therefore would add low-frequency variability of a different sort. In particular, what would the effect be on the already temporallychaotic oscillations which possess intrinsic low-frequency variability.

A primary inadequacy of the model used here is that it has no vertical structure. Although using a quasi-geostrophic layer model would not ease the burden of eddies having to flux the vorticity to the boundary, it would introduce new sources of eddies through baroclinic instability, potentially affecting the eddies formation and efficiency. Another significant effect of the introduction of vertical structure would 
be the requirement of satisfaction of a buoyancy budget (or salt and heat for that matter). The uniform density of the model used here requires only a satisfaction of mass conservation. Radko and Marshall (2002) and Marshall et al. (2002) have demonstrated that eddies may play a significant role in the distribution of heat as well as vorticity. What happens when if the requirements of fulfilling the heat budget are at odds with fulfilling the vorticity budget?

\subsubsection{Eddy Parameterization}

Finally, some of the results of this thesis could be useful for development of eddy parameterization.

\section{Homoparic Solutions}

The existence of the homoparic solutions to the single-gyre calculation indicates that in this instance, the eddy parameterization worked very well. That is, if two calculations with different $\operatorname{Re}($ int $)$ and $\operatorname{Re}(b d y)$ have almost the same time-mean because the eddies and the friction have changed in complementary ways, that is a very successful eddy parameterization! However, there were no obvious clues as to how to choose the value of $\operatorname{Re}(\mathrm{int})$ and $\operatorname{Re}(\mathrm{bdy})$. It seems that a much larger swath of parameter space would need to be explored to find this relation and understand it. At the present time computers are not fast enough to run the hundreds of calculations and at the higher resolutions needed to more fully map the functional relationship. The problem is one familiar to number theorists, many different sequences of small numbers seem the same at first.

The homoparic equilibria also present an opportunity for an interesting experiment. Since the time-mean of these calculations is very similar, they represent an effective parameterization of the mean effects of eddies. However, I have noted that the spin-up times of the different pair members is quite different; the member with larger Re(int) is slower to spin-up. The experiment, then, is to comparatively study 
the response of these pair members to time-dependent forcing. If the eddies are slower to respond than the linear viscosity, just what temporal forcing do the resolved eddies not resolve? Is one solution or the other more prone to state transitions under variable forcing?

\section{The Residual Mean Circulation}

Alan Plumb has suggested that perhaps a cleaner physical interpretation of eddy phenomena such as the homoparic solutions lies in choosing another definition of eddy. As eddies are defined here, they mix both along and across the vorticity gradients. By redefining the 'mean' about which the eddies are perturbations to include the along-gradient portion of what is now eddy flux, it is possible that one could be much more precise about the effects of the eddies.

One particularly promising idea is to redefine the mean so that the eddy fluxes are by definition down the mean gradient. This involves wrapping all of the skew components of $\overline{\mathbf{u}^{\prime}} \zeta^{\prime}$ into what is considered the mean flow. The remaining, or residual, eddy fluxes can then be studied by the distribution of a scalar quantity $\kappa: \overline{\mathbf{u}^{\prime}} \zeta^{\prime}=$ $-\kappa(x, y) \nabla \bar{\zeta}$. Since the model here used just such a form for the frictional fluxes, it would be quite interesting to be able to directly compare the resulting eddy fluxes from a change in the viscosity.

In some physical systems, using a well-chosen residual mean formulation actually results in a closed set of equations which can be solved without ever having knowledge of the Eulerian mean. However, this usually involves redefining mean to incorporate some features of the buoyancy budget, not to eliminate the skew fluxes as described in the preceding paragraph. In the homogeneous model, there is no buoyancy budget and at the present time, it is not clear how to formulate a closed residual mean formulation which eliminates the skew fluxes from the Reynolds vorticity flux. Thus, the residual mean formulation as currently understood would be only a diagnostic calculation, not a closed system of equations which could be solved without knowledge of the Eulerian 
mean.

\subsection{Final Remarks}

This dissertation has explored mechanisms by which closed interior streamlines can be constantly forced, yet separated from the area where vorticity is ultimately removed from the basin. I hope that it has helped resolve the long-standing puzzle of how the circulation strength can be controlled when much of the circulation receives an input of vorticity but no obvious output. In this case, an eddy flux of vorticity plays the critical role of communicating the vorticity between these isolated regions and the regions where the vorticity is ultimately removed. It has been clearly demonstrated here that both the eddy flux and the ultimate removal are critical, and without either one the vorticity is trapped in the basin or in an interior streamline and the circulation strength increases vastly beyond what is believable for a model of the real ocean.

Although the specific model used here is barotropic and employs a boundaryenhanced viscosity as a parameterization of the mechanism for removal of vorticity, it has direct relevance to an equivalent barotropic or upper moving layer of a baroclinic fluid with essentially any mechanism for vorticity removal. I have demonstrated that the mean flow need not enter this region of vorticity removal, so processes limited to a narrow region outside of the main flow are acceptable. Furthermore, this approach has allowed a resolution to many of the technical issues surrounding the traditional models, such as the difficulty of reattaching Charney's boundary layer to the interior flow and the mechanism of westward intensification with a relatively inviscid basin interior.

Along the way, the model had enough interesting behaviors to warrant its study regardless of whether it truly describes what controls the circulation strength in the

real ocean. The counter-rotating gyres, presence of basin modes, and development of temporal complexity with increasing nonlinearity all present stimulating ideas that 
are likely to have analogues or application in the real ocean.

I have certainly enjoyed and learned from doing this research and recording it here. I hope that you have enjoyed and learned from reading it. 


\section{Appendix A}

\section{Properties of the Chebyshev Polynomials}

\section{A.1 Definitions and Basic Identities}

The Chebyshev Polynomials may be defined as

$$
T_{n}(x)=\cos \left[n \cos ^{-1}(x)\right]
$$

From this definition, the first few polynomials are easily determined.

$$
\begin{aligned}
T_{0}(x) & =1 \\
T_{1}(x) & =x \\
T_{2}(x) & =2 x^{2}-1, \\
T_{3}(x) & =4 x^{3}-3 x, \\
T_{4}(x) & =8 x^{4}-8 x^{2}+1, \\
& \cdots \\
T_{n+1}(x) & =2 x T_{n}(x)-T_{n-1}(x)
\end{aligned}
$$


The orthogonality relation is

$$
\int_{-1}^{1} \frac{T_{i}(x) T_{j}(x)}{\sqrt{1-x^{2}}} d x= \begin{cases}0 & \text { if } i \neq j \\ \pi / 2 & \text { if } i=j \neq 0 \\ \pi & \text { if } i=j=0\end{cases}
$$

The zeros of $T_{n}(x)$ are located at

$$
T_{n}(x)=0 \rightarrow \quad x=\cos \left[\frac{\pi\left(k-\frac{1}{2}\right)}{n}\right] \quad k=1,2, \ldots, n
$$

When the polynomials are summed over the points where they are zero, they obey a discrete orthogonality relation. If $x_{k}$ are the $m$ zeros of $T_{m}(x)$, and $i, j<m$, then

$$
\sum_{k=1}^{m} T_{i}\left(x_{k}\right) T_{j}\left(x_{k}\right)= \begin{cases}0 & \text { if } i \neq j \\ m / 2 & \text { if } i=j \neq 0 \\ m & \text { if } i=j=0\end{cases}
$$

The extrema of $T_{n}(x)$ are located at

$$
\left\|T_{n}(x)\right\|=1 \rightarrow x=\cos \left[\frac{\pi k}{n}\right] \quad k=0,1,2, \ldots, n
$$

When the polynomials are summed over the points where they are extremal, they obey a discrete orthogonality relation. If $x_{k}$ are the $m$ extrema of $T_{m}(x)$, and $i, j<m$, then

$$
\sum_{k=0}^{m} T_{i}\left(x_{k}\right) T_{j}\left(x_{k}\right)=\left\{\begin{array}{ll}
0 & \text { if } i \neq j \\
\frac{m+2}{2} & \text { if } i=j \neq 0 \\
m+1 & \text { if } i=j=0
\end{array} .\right.
$$

For the purposes of the expansion, consider a basin where the boundaries lie at $x= \pm 1$ and $y= \pm 1$. To ensure that the boundary conditions are satisfied, the 
following identities are useful.

$$
\begin{aligned}
T_{n}( \pm 1) & =( \pm 1)^{n} \\
\frac{d^{p}}{d x^{p}} T_{n}( \pm 1) & =( \pm 1)^{n+p} \prod_{k=0}^{p-1} \frac{n^{2}-k^{2}}{2 k+1}
\end{aligned}
$$

\section{A.2 Chebyshev Polynomial Expansion to Approx- imate Functions}

The relevance of the Chebyshev polynomials lies in using the orthogonality relations above to construct a series expansion. To approximate a function, $f(x)$, we assume a series expansion of the form

$$
f(x)=\left[\sum_{k=0}^{N-1} c_{k} T_{k}(x)\right]-\frac{c_{0}}{2}
$$

In this thesis, the method of collocation is used to determine the constants $c_{k}$. This means that $N$ points are chosen in $x$ where equation A.9 will hold exactly. It is clear from equations A. 6 and A.4 that the collocation points should be chosen to be the points where the Chebyshev polynomials are zero or extremal.

With the zeros as the collocation points, then the relation becomes

$$
c_{j}=\left[\sum_{k=1}^{N} f\left(\cos \left[\frac{\pi\left(k-\frac{1}{2}\right)}{N}\right]\right) \cos \left[\frac{\pi j\left(k-\frac{1}{2}\right)}{N}\right]\right] .
$$

Thus, given the values of $f$ at the zeros of the Chebyshev polynomials, only a cosine transform is needed to determine the coefficients. In this thesis, however, a slightly different version is more useful because we would like the endpoints $x= \pm 1$ to be collocation points.

Choosing the collocation points to be at the extrema of the Chebyshev polynomials, the boundaries are included in the grid, so boundary conditions are easily 
interpreted and verified. This grid is called the Gauss-Lobatto grid. All of the Chebyshev calculations in this thesis were done on a Gauss-Lobatto grid. On the Gauss-Lobatto grid, the coefficients may be determined using an alternate form of the cosine transform.

$$
\widehat{f_{k}}=\left[\sum_{j=0}^{N} f_{j} \cos \left[\frac{\pi j k}{N}\right]\right]-\frac{1}{2}\left[f_{0}+(-1)^{k} f_{N}\right]
$$

Note that the inverse of this transform is itself times $2 / N$, which follows from the equation A.4. Using this fact to convert to and from the Chebyshev coefficients,

$$
\begin{aligned}
f\left(x_{j}\right) & =\widehat{c_{k j}}+(-1)^{j} \frac{c_{N}}{2}, \\
c_{k} & =\frac{2}{N} \widehat{f\left(x_{j}\right)_{k}}-\frac{2}{N} \frac{\widehat{f\left(x_{j}\right)_{N}}}{N+\left(\widehat{(-1)^{j}}{ }_{N}\right.}\left(\widehat{-1)^{j}}{ }_{k} .\right.
\end{aligned}
$$

In this thesis the cosine transform from equation A.11 was implemented using the fast Fourier transform, producing an efficient method of transforming back and forth from physical variables to Chebyshev coefficients.

It is possible to calculate $(\widehat{-1})_{k}^{j}$ for any $N$. Because of the heavy symmetry of cosine about 0 to $\pi$, the following applies

$$
\begin{aligned}
\widehat{(-1)^{j}}{ }_{k} & =\left\{\begin{array}{ll}
0 & \text { if } k \neq N \\
N & \text { if } k=N
\end{array} .\right. \\
f\left(x_{j}\right) & =\widehat{c_{k}}+(-1)^{j} \frac{c_{N}}{2} \\
c_{k} & = \begin{cases}\left.\frac{2}{N} \widehat{f\left(x_{j}\right.}\right)_{k} & \text { if } k \neq N \\
\left.\frac{1}{N} \widehat{f\left(x_{j}\right.}\right)_{N} & \text { if } k=N\end{cases}
\end{aligned}
$$

Using these results, the transformation to physical space to perform operations is easy, but it will often be helpful to perform operations in Chebyshev space as well. 
Most importantly, the derivative of a Chebyshev expansion can be calculated

$$
\begin{aligned}
\frac{d^{q}}{d x^{q}} f(x) & =\sum_{n=0}^{\infty} a_{n}^{(q)} T_{n}(x)-\frac{a_{0}^{(q)}}{2}, \\
a_{n-1}^{(q)}-a_{n+1}^{(q)} & =2 n a_{n}^{(q-1)}, n \geq 1, \\
a_{n}^{(q)} & =2 \sum_{p=n+1, p+n=o d d}^{\infty} p a_{p}^{(q-1)} .
\end{aligned}
$$

The truncation of these equations is carried out by setting $a_{p}^{(q-1)}=0$ for $p>N$. This is equivalent to setting $a_{n}^{(q)}=0$ for $n \geq N$ in the recursion equation. This truncation represents the degree of approximation with differentiation. An Nth degree polynomial approximation of the original function is reduced to an $(N-1)$ th degree approximation of the function derivative.

The indicial equation A.18 for the derivative of a function given that function was used to generate all of the derivatives in the Chebyshev polynomial models in this thesis. This equation was also used to integrate spatially when necessary.

\section{A.3 2-dimensional Expansions}

As the models in this thesis are two-dimensional, the Chebyshev transformation is on a function of the form $f(x, y)$. This is effected by transforming along one coordinate first, and then transforming along the other.

$$
\begin{aligned}
f(x, y)= & \sum_{j=0}^{M} c_{j}(y) T_{j}(x)-\frac{c_{0}(y)}{2}=\sum_{j=0}^{M^{\prime}} c_{j}(y) T_{j}(x) \\
f(x, y)= & \sum_{j=0}^{M} \sum_{k=0}^{N} c_{j k} T_{j}(x) T_{k}(y)-\sum_{j=0}^{M} T_{j}(x) \frac{c_{j 0}}{2}-\sum_{k=0}^{N} T_{k}(y) \frac{c_{0 k}}{2}+\frac{c_{00}}{4}, \\
& =\sum_{j=0}^{M^{\prime}} \sum_{k=0}^{N^{\prime}} c_{j k} T_{j}(x) T_{k}(y) .
\end{aligned}
$$

The notation of a prime following the sum $\left(\Sigma^{\prime}\right)$ is used to denote a sum over the series followed by subtraction of half of the first term in the series. This expansion 
and its inverse are easily accomplished by taking the Chebyshev expansion along one coordinate using the first expansion and then expanding the resulting Chebyshev coefficients along the other coordinate.

\section{A.4 Boundary Conditions}

In the Chebyshev expansion, the boundary conditions can take a complicated form. This section presents the forms used in this thesis.

If the basin boundaries lie at $x= \pm 1$ and $y= \pm 1$, then for a single-gyre calculation, no-normal-flow is one boundary condition. This means that the gradient of the streamfunction along the boundary must vanish. Using the formula derived for the values at the boundaries (equation A.8), we can find the boundary conditions on the coefficients $\psi_{j k}$ of a Chebyshev expansion of the streamfunction $\psi(x, y)$.

$$
\begin{aligned}
& \psi(x, y)=\sum_{j=0}^{M^{\prime}} \sum_{k=0}^{N '} \psi_{j k} T_{j}(x) T_{k}(y) . \\
& 0=\sum_{j=0}^{M^{\prime}} \sum_{k=0}^{N \prime} \psi_{j k, x} T_{j}(x)( \pm 1)^{k}, \\
& \psi_{(j-1) k, x}-\psi_{(j+1) k, x}=2 j c_{j k}, \quad \forall: j \in\{1,2, \ldots, M\} .
\end{aligned}
$$

Since the $T_{j}(x)$ are orthogonal,

$$
\begin{aligned}
& 0=\sum_{k=0}^{N^{\prime}} \psi_{j k, x}( \pm 1)^{k}, \\
& \psi_{(j-1) k, x}-\psi_{(j+1) k, x}=2 j \psi_{j k},
\end{aligned} \quad \forall: j \in\{1,2, \ldots, M\} .
$$

Likewise for the eastern and western boundaries,

$$
\begin{aligned}
& 0=\sum_{j=0}^{M^{\prime}} \psi_{j k, y}( \pm 1)^{j}, \\
& \psi_{j(k-1), y}-\psi_{j(k+1), y}=2 k \psi_{j k},
\end{aligned} \quad \forall: k \in\{1,2, \ldots, N\} .
$$


There are four more boundary conditions to prevent outflow at the corners of the basin, namely, that the value of the streamfunction should be equal on all four walls.

There is an alternate method for setting the no-normal-flow condition. Since the tangential derivative along the boundary is supposed to vanish, we can integrate around the boundary. Clearly, we see that the value of $\psi$ will be constant along the boundary. This constant may vary in time (if time dependence is important), but for the fixed-depth model used in this thesis this is not physically important. To set the boundary values to zero,

$$
\begin{aligned}
& 0=\sum_{j=0}^{M^{\prime}} \sum_{k=0}^{N \prime} \psi_{j k}( \pm 1)^{j} T_{k}(y) \\
& 0=\sum_{j=0}^{M^{\prime}} \sum_{k=0}^{N^{\prime}} \psi_{j k} T_{j}(x)( \pm 1)^{k}
\end{aligned}
$$

Again, we may use the orthogonality of the polynomials to insist that one of the two sums vanishes term by term.

$$
\begin{aligned}
& 0=\sum_{j=0}^{M^{\prime}} \psi_{j k}( \pm 1)^{j}, \quad \forall: k \in\{0,1, \ldots, N\} . \\
& 0=\sum_{k=0}^{N^{\prime}} \psi_{j k}( \pm 1)^{k}, \quad \forall: j \in\{0,1, \ldots, M\} .
\end{aligned}
$$

The other boundary conditions have a similar form.

No-slip boundary conditions are set by

$$
\begin{aligned}
& x= \pm 1 \longrightarrow 0=\sum_{j=0}^{M^{\prime}} j^{2} \psi_{j k}( \pm 1)^{j}, \quad \forall: k \in\{0,1, \ldots, N\} \\
& y= \pm 1 \longrightarrow 0=\sum_{k=0}^{N^{\prime}} k^{2} \psi_{j k}( \pm 1)^{k}, \quad \forall: j \in\{0,1, \ldots, M\}
\end{aligned}
$$


No-stress boundary conditions are set by

$$
\begin{gathered}
x= \pm 1 \longrightarrow 0=\sum_{j=0}^{M^{\prime}} \frac{j^{2}\left(j^{2}-1\right)}{3} \psi_{j k}( \pm 1)^{j}, \quad \forall: k \in\{0,1, \ldots, N\} \\
y= \pm 1 \longrightarrow 0=\sum_{k=0}^{N^{\prime}} \frac{k^{2}\left(k^{2}-1\right)}{3} \psi_{j k}( \pm 1)^{k}, \quad \forall: j \in\{0,1, \ldots, M\} .
\end{gathered}
$$




\section{Appendix B}

\section{The Steady-State Numerical}

\section{Models}

The steady-state calculations in this thesis were generated using one of two numerical models. This appendix briefly describes these models.

\section{B.1 Newton's Method}

The method used to solve for steady-state solutions is Newton's method. It consists of solution of a function by iteratively solving linear approximations.

\section{B.1.1 One-Dimensional Newton's Method}

In one dimension, Newton's method is very simple and converges quadratically when the root is nearby.

Suppose $F(y)=0$ is the equation to solve and $y$ is the solution. Suppose an initial guess $x$ is chosen. An estimate of $y$ using $x$ and a Taylor series expansion is generated.

$$
F(y)=0
$$




$$
\begin{aligned}
F(y) & =F(x)+F^{\prime}(x)(y-x)+F^{\prime \prime}(y)(y-x)^{2}+\ldots \\
y & =x+\frac{F(y)-F(x)}{F^{\prime}(x)}+O\left[(y-x)^{2}\right] \\
y & \approx x+\frac{-F(x)}{F^{\prime}(x)}
\end{aligned}
$$

In Newton's method this procedure is performed iteratively. That is, calculate an estimate of $y$ using equation B.2 from an initial guess $x$. Then, use the estimate of $y$ as the new guess, and iterate. The accuracy at each step is always calculated as $F(x)$. With iteration, this can be made quite small.

There are two subtleties of Newton's method in one dimension. First, there is no way of determining the number of roots during solution. If there is at least one root and the function is smooth, Newton's method will probably find it, but if there is more than one root, the convergence will be determined by the initial guess. Usually, if one knows something about the roots, the initial guesses can be chosen close to them, thus increasing the likelihood of convergence to the desired root. Second, it is possible that the derivative, $F^{\prime}(x)$, will vanish during the course of the solution. In this case, one perturbs the guess slightly and tries the iteration again.

\section{B.1.2 Multi-dimensional Newton's Method}

In a very similar method to that of the one dimensional Newton's method, we can iteratively solve a multi-dimensional nonlinear set of equations. This is achieved via ${ }^{1}$

$$
\begin{array}{rll}
\left.F_{i}\right|_{y} & = & 0, \\
\left.F_{i}\right|_{y} & = & \left.F_{i}\right|_{x}+\left.F_{i, j}\right|_{x}\left(y_{j}-x_{j}\right)+\left.F_{i, j k}\right|_{x}\left(y_{j}-x_{j}\right)\left(y_{k}-x_{k}\right)+\ldots, \\
-\left.\left.F_{i}\right|_{x} \approx F_{i, j}\right|_{x} & \left(y_{j}-x_{j}\right) .
\end{array}
$$

\footnotetext{
${ }^{1}$ Here I am using Einstein summation to imply that repeated indices are summed over all the dimensions present
} 
The iterative procedure involves the solution of a matrix equation involving the Jacobian matrix $F_{i, j}$. The solution is

$$
y_{j} \approx x_{j}-\left(F_{i, j}(x)\right)^{-1} F_{i}(x)
$$

There are many subtleties in the multi-dimensional case. A few of them relate directly to the matrix inversion. Of course, because we have to solve the matrix equation, $F_{i}$ must be a vector of the same dimension as $x_{i}$. That is, we must have as many equations as unknowns. Also, the equations must be linearly independent, which is equivalent to saying that the Jacobian must be nonsingular at every $x$ the iteration strikes. The latter is ameliorated by noting that if the Jacobian turns out to be singular, it is likely to be only a local effect, so perturbing the $x$ a bit usually recovers invertability.

Even if the matrix inversion works, there is another subtlety to the multi-dimensional Newton's method: lack of guaranteed convergence. In the one dimensional case, as long as $F$ decreased, the iteration proceeds toward a root. In the multi-dimensional case, it is not so simple. It is very easy to become trapped in a local minima. For this reason, the models in this thesis employ a backtracking routine to the Newton's method. This routine ensures that if the Newton's method step doesn't decrease $F_{i} F_{i}$, then only a percentage of that step is taken. The percentage is shrunk until $F_{i} F_{i}$ decreases or a minimum step length is reached. If the minimum step length is reached, then it is assumed that the solution is trapped in a local minimum, and the full step length is used. The benefit of this kind of backtracking is that it is numerically inexpensive (relative to the matrix equation solution for a full Newton's step) and it may increase the speed of convergence when far away from a solution. It also has the added bonus of discovering when the solver is trapped in a local minimum.

This method is essentially that of the Newton's Method with backtracking from the numrec. Although the code is not directly implemented, I have done little to change the methodological essence. 


\section{B.1.3 Evaluation of the Jacobian}

The Jacobian $F_{i, j}$ is estimated using a simple forward difference scheme given in Press et al. (1992).

$$
\left.F_{i, j}\right|_{x} \approx \frac{\left.F_{i}\right|_{x+d x}-\left.F_{i}\right|_{x}}{d x_{j}}
$$

The perturbation $d x$ is tangent to the $j_{t h}$ component of $x$. A very small fraction of the $j^{\text {th }}$ component of $x$ is used as the magnitude $|d x| j$ for each $j$. This is a dependable method for determining the Jacobian, but it is expensive. It requires that the function $F$ be called $N^{2}+1$ times for each determination of the Jacobian, where $N$ is the number of degrees of freedom of $x$.

\section{B.2 Finite Difference Model}

The finite difference numerical model was constructed from the homogeneous ocean model's partial differential equation.

$$
F=-\delta_{I}^{2} J(\psi, \zeta)-\psi_{, x} w_{E}-\nabla_{i}\left[\delta_{S}\left(\psi_{, i}-\epsilon_{z i k} \psi_{, k}\right)\right]+\nabla_{i} \delta_{M}^{3} \zeta_{, i}
$$

The discretization was carried out by finite differences on an irregular grid. The gradients were calculated as an average of the differences to either direction and the second derivatives were taken as centered differences. As an example, the $i$ th grid point in $x$ would have derivatives of the following form

$$
\begin{aligned}
\frac{\partial \psi}{\partial x} & \approx \frac{\psi\left(x_{i}\right)-\psi\left(x_{i-1}\right)}{2\left(x_{i}-x_{i-1}\right)}+\frac{\psi\left(x_{i+1}\right)-\psi\left(x_{i}\right)}{2\left(x_{i+1}-x_{i}\right)} \\
\frac{\partial^{2} \psi}{\partial x^{2}} & \approx\left[\frac{\psi\left(x_{i}\right)-\psi\left(x_{i-1}\right)}{\left(x_{i}-x_{i-1}\right)}-\frac{\psi\left(x_{i+1}\right)-\psi\left(x_{i}\right)}{\left(x_{i+1}-x_{i}\right)}\right] \frac{2}{\left(x_{i+1}-x_{i-1}\right)}
\end{aligned}
$$

This method makes the second derivatives a lower order approximation than the first derivatives, it allows the boundary conditions to be set more easily. 
The grid was generated using

$$
x_{i}=\frac{\tan \left[\pi \rho\left(\xi_{i}-\xi_{0}\right) / 2\right]-\tan \left[\pi \rho\left(-\xi_{0}\right) / 2\right]}{\tan \left[\pi \rho\left(1-\xi_{0}\right) / 2\right]-\tan \left[\pi \rho\left(-\xi_{0}\right) / 2\right]}
$$

This formula gives a smoothly-varying grid of $x_{i}$ from 0 to 1 from a uniform grid of $\xi_{i}$ between 0 and 1 . Most of the grid points cluster near $\xi_{0}$ and a variable density of grid points is governed by $\rho$, a parameter between 0 and 1 . The calculations presented in the thesis were made with values of $\rho$ and $\xi_{0}$ that were approximately 0.65 and 0.1 , respectively, for a grid with 35 grid points in each direction.

The boundary conditions were implemented by defining ghost cells outside of the domain. The differentiation was then carried out to the edge of the domain, and these ghost cells assured the satisfaction of the boundary conditions there. For the problem with bottom friction only, the ghost cells were needed only at the boundary to set the value of $\psi$ to zero to ensure no-normal-flow.

For the problems with lateral friction, here is an example of how the ghost cells were set. The ghost cell was located at $-d x$, where $d x$ was the location of the first interior grid point. For no-slip boundary conditions at the western boundary $(x=0)$, the value of $\psi(-d x)$ was set to 0 . For slip boundary conditions, the value of $\psi(-d x)$ was set to $\psi(d x)$

The Newton's method minimized the value of $\sum\left(F\left(x_{i}, y_{j}\right)^{2}\right)$ where the sum is taken over every interior grid point. The boundary conditions on $\psi$ were set before $F$ was evaluated.

\section{B.3 Chebyshev Pseudo-Spectral Model}

The finite difference numerical model was constructed from the homogeneous ocean model's partial differential equations which was discretized using the Chebyshev transforms discussed in appendix A. The discretization resulted in the following equations 
to solve.

$$
\begin{aligned}
G= & -\delta_{I}^{2} C\left(C^{-1}\left(C(\psi)_{, x}\right) C^{-1} \nabla^{2} C(\psi)_{, y}-C^{-1}\left(C(\psi)_{, y}\right) C^{-1} \nabla^{2} C(\psi)_{, x}\right)-C(\psi)_{, x} \\
& +C\left(w_{E}\right)-\nabla_{i}\left[\delta_{S}\left(C(\psi)_{, i}-\epsilon_{z i k} C(\psi)_{, k}\right)\right]+\nabla_{i} \delta_{M}^{3} \nabla^{2} C(\psi)_{, i}
\end{aligned}
$$

The Chebyshev tranform of the function $f$ is written as $C(f)$, and $C^{-1}(f)$ is the inverse transform.

Since the taking the derivative lowers the order of the Chebyshev expansion, the lateral friction term in the field equations was zero for the four highest-order Chebyshev polynomials in each direction. For these remaining degrees of freedom, the boundary conditions were used to set their value.

It is critical to evaluate the lowest modes of the field equations for these models, because the single-gyre forcing projects onto a single Chebyshev mode.

The Gauss-Lobatto grid was used, the basin boundaries were included as grid points for the collocation. This made the implementation of the boundary conditions quite easy. The boundary conditions were set as described in the previous appendix, and slip or no-slip or mixed boundary conditions were implemented without difficulty.

If the Chebyshev expansion had $N$ polynomials in $y$ and $M$ polynomials in $x$, then there are $N M$ degrees of freedom. The field equations represented $(N-4)(M-4)$ equations. There were 2 boundary conditions in each direction on each boundary, so that is $4 N+4 M$ boundary conditions. There are therefore $(N-4)(M-4)+4 N+4 M=$ $N M+16$ total equations. Therefore, 16 of the equations were redundant, which is clearly because the boundary conditions at the corners are evaluated along the boundary in each direction. Removing these redundant equations, there are $N M$ equations in $N M$ unknowns.

The Newton's method proceeded as follows. An initial guess for $\psi$ was proposed. The boundary conditions were used to set the lowest four Chebyshev modes in each direction of $\psi$. Then the $(N-4)(M-4)$ field equations were minimized using the 


\begin{tabular}{|c|c|c|c|c|c|c|c|}
\hline \hline Location & $\delta_{I}$ & $\delta_{M}($ int $)$ & $\delta_{M}(\mathrm{bdy})$ & $\delta_{S}$ & $L_{x}$ & $L_{y}$ & Resolution \\
\hline \hline Fig. 1-1a & 0 & 0 & 0 & 0.04 & 1 & 1 & $34 \times 35$ (NFD) \\
\hline Fig. 1-1b & 0.04 & 0 & 0 & 0.04 & 1 & 1 & $34 \times 35$ (NFD) \\
\hline Fig. 1-1c & 0.08 & 0 & 0 & 0.04 & 1 & 1 & $34 \times 35(\mathrm{NFD})$ \\
\hline Fig. 1-1d & 0.1 & 0 & 0 & 0.04 & 1 & 1 & $34 \times 35(\mathrm{NFD})$ \\
\hline Fig. 1-2d & 0 & 0.05 & 0.05 & 0 & 1 & 1 & $49 \times 49(\mathrm{C})$ \\
\hline Fig. 1-2d & 0.05 & 0.05 & 0.05 & 0 & 1 & 1 & $49 \times 49(\mathrm{C})$ \\
\hline Fig. 1-2d & 0.0625 & 0.05 & 0.05 & 0 & 1 & 1 & $49 \times 49(\mathrm{C})$ \\
\hline Fig. 1-2d & 0.08125 & 0.05 & 0.05 & 0 & 1 & 1 & $49 \times 49(\mathrm{C})$ \\
\hline
\end{tabular}

Table B.1: This table presents the parameter settings for all of the steady-state calculations presented in this dissertation. Resolution is given in number of grid points (Chebyshev modes) in $y$ by number in $x$. NFD is a nonuniform grid finite difference scheme as in section B.2. $\mathrm{C}$ is Chebyshev polynomial expansion as in section B.3.

Chebyshev coefficients of $\psi$ for modes greater than 4 . Each time a new version of the Chebyshev coefficients was generated by the Newton's method, the lowest 4 modes of the Chebyshev coefficients were calculated from the boundary conditions.

The solutions to the steady-state problem are relatively smooth, so the Chebyshev coefficients should rapidly decrease with increasing mode. Therefore, the metric for convergence of $G$ might be chosen to decrease with mode number, so that convergence to lower modes was deemed more important than higher ones. Therefore sometimes the Newton's method was used to minimize $\sum(G(n, m) /(n m))^{2}$ where $n$ and $m$ are the mode number and the sum is taken over all modes where either $n$ or $m$ is greater than 4. In practice, minimizing $\sum(G(n, m))^{2}$ yielded comparable performance so long as the solution was adequately resolved, so this was used instead.

The advantage of minimizing the Chebyshev transform of the field equations rather than the value of the function at grid points within the space was that the number of Fourier transforms required to evaluate the Jacobian was minimized. However, it is likely that the convergence might be more rapid in a model which minimized the values in physical space. 


\section{Appendix $\mathrm{C}$}

\section{The Time-Dependent Numerical}

\section{Model}

The majority of the modeling in the thesis was calculated using a time-integrating numerical model specifically for the purpose. This appendix describes the model.

The nonlinear terms and the variable viscosity friction terms were evaluated by pseudo-spectral collocation. A major difficulty with many numerical models, especially those employing a hyper-viscosity, is that the number of derivatives required to evaluate these terms is unrealistically large. Therefore, the errors in the lowerderivative terms can end up resembling higher-order derivatives in practice. Because the experimental design here required accurate evaluation of the highest derivatives, some care had to be taken with the numerical approach.

\section{C.1 Implementing The Field Equations}

The field equation for this model was the homogeneous model vorticity equation. It was solved in a four-stage process.

The time integration was done by a second-order Adams-Bashforth method for all terms except the lateral friction. For stability, it was necessary to evaluate the 
friction in two parts, the first was the frictional term with constant viscosity equal to the maximum found in the basin. This term was evaluated implicitly with a modified Crank-Nicholson scheme. The corrections to the constant viscosity were evaluated explicitly by Adams-Bashforth. This use of an implicit integration method in this manner is recommended by Gottlieb and Orszag (1981) and Orszag and Israeli (1974) for solving variable-viscosity problems with Chebyshev polynomials. The choice of Adams-Bashforth and modified Crank-Nicholson schemes was recommended in Canuto et al. (1988).

These methods result in the following time-stepping scheme,

$$
\begin{aligned}
{\left[1-\theta d t \max \left(\delta_{M}^{3}\right) \nabla^{2}\right] \zeta_{0}(t+d t)=} & {\left[1+(1-\theta) d t \max \left(\delta_{M}^{3}\right) \nabla^{2}\right] \zeta(t)-\sin (\pi y) } \\
& +d t\left[\frac{3 X(t)}{2}-\frac{X(t-d t)}{2}\right] \\
\zeta(t+d t)= & \zeta_{0}(t+d t)+\zeta_{H} \\
\zeta(t+d t)= & \nabla^{2} \psi(t+d t) \\
\theta & \equiv 0.50001
\end{aligned}
$$

The explicit terms are given by

$$
\begin{aligned}
X(\tau)= & \frac{\partial}{\partial x}\left[\left(\delta_{M}^{3}-\max \left(\delta_{M}^{3}\right)\right) \frac{\partial}{\partial x} \zeta(\tau)+\zeta(\tau) \frac{\partial}{\partial y} \psi(\tau)-\psi(\tau)\right] \\
& +\frac{\partial}{\partial y}\left[\left(\delta_{M}^{3}-\max \left(\delta_{M}^{3}\right)\right) \frac{\partial}{\partial y} \zeta(\tau)-\zeta(\tau) \frac{\partial}{\partial x} \psi(\tau)\right]-\delta_{S} \zeta(\tau)
\end{aligned}
$$

All of the above equations were solved while the variables were expanded in Chebyshev polynomials. The differentiation was done using the properties of the Chebyshev polynomial expansion. Only the products in the explicit terms for the advective terms and the variable viscosity friction terms were calculated on the collocation points of the Chebyshev polynomials. Once the products were formed, the results were projected back into Chebyshev polynomials. 


\section{C.2 Inversion of Poisson and Helmholtz Equations}

In each time step, the Helmholtz operator on the left-hand side of equation C.1 had to be inverted to find the new $\zeta_{0}$, and the Poisson operator in equation C. 3 had to be inverted to find the new $\psi$. The inverses of these operators were determined at the beginning of each calculation, and the inversion was then performed by matrix multiplication.

If the Chebyshev coefficients are expressed in matrix format, then all linear operations can be performed by matrix multiplication. In this model, the columns represent the $\mathrm{n}$ mode and the rows represented the $\mathrm{m}$ mode. Thus, the Helmholtz equation could be represented as

$$
\begin{aligned}
\left(1+\lambda \nabla^{2}\right) \zeta & =R \\
{\left[1+(1-\theta) d t \max \left(\delta_{M}^{3}\right) \nabla^{2}\right] \zeta(t)-\sin (\pi y) } & \equiv R \\
{[\zeta]+\lambda\left(\left[\frac{\partial^{2}}{\partial y^{2}}\right][\zeta]+[\zeta]\left[\frac{\partial^{2}}{\partial x^{2}}\right]\right) } & =[R]
\end{aligned}
$$

Brackets denote the matrix representation of the Chebyshev coefficients of the variables or matrix representation of the operators acting on the matrices. Note that left-multiplication of a Chebyshev expansion operates on the $y$ modes of the Chebyshev expansion while right-multiplication operates on the $x$ modes.

The differential operators include the slip boundary condition $(\zeta=0)$. Because the differentiation of Chebyshev expansion leaves the highest modes zero, the matrix representation of differentiation will be singular. Including the boundary condition makes them non-singular.

To solve this equation, at the beginning of the calculation, the difference matrices are diagonalized. This is allowed because they are non-singular, so long as the boundary conditions are included. They are also relatively well-conditioned, so a standard 
eigenvector package from the Compaq $\mathrm{dxml}$ library was used.

$$
\begin{gathered}
{[V(y)][\lambda(y)][V(y)]^{-1}=\left[\frac{\partial^{2}}{\partial y^{2}}\right]} \\
{[V(x)][\lambda(x)][V(x)]^{-1}=\left[\frac{\partial^{2}}{\partial x^{2}}\right]} \\
{[V(y)][V(y)]^{-1}[\zeta][V(x)][V(x)]^{-1}+\lambda\left([V(y)][\lambda(y)][V(y)]^{-1}[\zeta]+[\zeta][V(x)][\lambda(x)][V(x)]^{-1}\right)} \\
=[V(y)][V(y)]^{-1}[R][V(x)][V(x)]^{-1}
\end{gathered}
$$

The matrices $[V(x)]$, and $[V(y)]$ have the eigenvectors as columns and the matrices $[\lambda(x)]$, and $[\lambda(y)]$ are diagonal matrices with the eigenvalues of the differentiation operators as the diagonal entries. By multiplying through by some of the matrices, a form of this equation which is diagonal can be found.

$$
\left[[V(y)]^{-1}[\zeta][V(x)]\right]_{n m}=\frac{\left[[V(y)]^{-1}[R][V(x)]\right]_{n m}}{1+\lambda\left([\lambda(y)]_{n n}+[\lambda(y)]_{m m}\right)}
$$

Subscripts denote column and row indices. Once $[V(y)]^{-1}[R][V(x)]$ is calculated, it is easy to solve for $[V(y)]^{-1}[\zeta][V(x)]$, which can be converted back to $\zeta$. Therefore, the Helmholtz equation can be solved in four matrix multiplications per time step.

The Poisson equation can be solved similarly. If the boundary condition of nonormal flow $(\psi=0)$ is imposed, the eigenvectors and eigenvalues of the differentiation operators are the same as those in used in the Helmholtz equation.

$$
\left[[V(y)]^{-1}[\psi][V(x)]\right]_{n m}=\frac{\left[[V(y)]^{-1}[\zeta][V(x)]\right]_{n m}}{[\lambda(y)]_{n n}+[\lambda(y)]_{m m}}
$$

Noting that the right-hand side of the Poisson equation is the same as the unknown in the Helmholtz equation, two matrix multiplications per time-step can be saved by solving these equations simultaneously.

Because the errors in matrix multiplication are cumulative, and the matrices multiplied here are large, the accuracy of these methods was checked at the beginning of each calculation by comparing the results from a forward and inverse calculation of 
the Helmholtz and Poisson operators. The errors were always less than one part in ten thousand.

The speed of solution of the entire method is governed by the number of matrix multiplications involved in solving the Helmholtz and Poisson equations. The method used results in six matrix multiplications per time step. There are three known methods to increase the speed of this step.

First, because differentiating the Chebyshev polynomials twice doesn't couple the even and odd Chebyshev modes, they can be treated separately. Although this increases the number of matrix multiplications, the size of each matrix in the multiplication is reduced. This results in a net gain in number of operations.

Second, instead of using Chebyshev expansions in both directions, a Fourier sine series can be used to expand in the north-south direction because only slip boundary conditions are used there. Fourier series are not suitable for no-slip boundary conditions. Because the Fourier modes are the eigenmodes of differentiation, this reduces the number of matrix multiplications by half. However, the uniform collocation points in a Fourier representation are ill-suited for the single-gyre calculations performed here. Uniform collocation would, on the other hand, probably have been preferable for the double and two-gyre calculations.

Third, there is a method due to Haidvogel Haidvogel (1976), which reorganizes the equations for solution of the Poisson and Helmholtz equations which allows the number of matrix multiplications to be halved as well. This method was not used because it was more difficult to implement and check numerically.

\section{C.3 Setting the Boundary Conditions}

The boundary condition of impermeability ( $\psi=0$ on boundary) was implemented within the operator for inversion of the Poisson equation. The Helmholtz inversion automatically set a slip boundary condition ( $\zeta_{0}=0$ on boundary). The condition of 
no-slip was then found by adjustment with a homogeneous solution to the Helmholtz operator with inhomogeneous boundary conditions $\left(\zeta_{H} \neq 0\right.$ on eastern and western boundaries, $\left[1-\theta d t \max \left(\delta_{M}^{3}\right) \nabla^{2}\right] \zeta_{H}=0$ ). At each time-step, $\zeta_{H}$ was constructed by adding together members from a set of homogeneous solutions determined at the beginning of the calculation. The size of this set was precisely large enough to ensure that the velocity vanished at every collocation point along the eastern and western boundaries. This method for setting the boundary conditions is essentially the one used by Haidvogel (1976).

\section{C.4 Spinning up the Model}

The calculations are spun-up in such a way as to diminish the excitation of basin modes by impulsive initial conditions. To do this, the forcing was not applied impulsively, rather it was slowly increased linearly from zero to its final value. A bottom friction was applied over the same time period. The ramp-up period took place over a time-scale long when compared to the applied bottom frictional decay time. Implementation of this procedure significantly decreased the time for statistically steady solutions to be reached, and presumably made the calculations with intrinsic instability closer to the state reached after longer integration in time with impulsive forcing.

\section{C.5 Sources of Numerical Error}

The numerical method used was chosen to allow for sufficiently accurate differentiation to ensure that the variable viscosity chosen was accurately represented. The numerical errors associated with this method are unlike those present in a finitedifference calculation and so warrant mention. There are four primary categories of error in this methodology: precision truncation, spectral truncation, aliasing, and time-step errors. 
The spectral truncation errors, errors due to insufficient order of Chebyshev polynomials is small. This can be verified by the spectral decay of the polynomial coefficients (they should exponentially decrease after sufficient resolution is reached).

The aliasing errors are errors due to the method of collocation. The products for the explicit terms (both frictional and advective) should properly have smaller spatial scale than the functions being multiplied. Because the spectrum of Chebyshev polynomials does not resolve these smaller scales, the energy in these scales is erroneously placed (aliased) into larger scale polynomials. Because the polynomial coefficients decay exponentially, however, these effects will also decay exponentially with resolution. The size of these errors can be estimated from the spectrum, and they are less than $1 \%$ in all of the calculations. However, since the Chebyshev polynomials are global functions, these errors are present in regions where there is little else going on. These aliasing errors produce the grid-scale errors present in the southwest region of figure 2-6d.

The numerical truncation errors are errors due to the limited digits of precision of the machine used. The calculations were done in double precision to minimize these errors. However, because the inversion of the Helmholtz and Poisson operators required matrix multiplication, these errors are compounded and are likely to be on the order of $0.01 \%$.

Figure C-1 shows the spectral decay of the Chebyshev coefficients of the timemean vorticity in a number of calculations. The vorticity is not as difficult to resolve as the frictional term, and the time-mean is probably also easier to resolve than the snapshot vorticity field. However, this is a fair representation of the magnitude of aliasing, truncation, and precision errors.

Aliasing errors are apparent in figure C-1 near the tail of the distributions, where the Chebyshev coefficients begin to rise. The small magnitude of these errors is apparent, however.

Truncation errors are evident in figure C-1 as well. If more modes were included, 


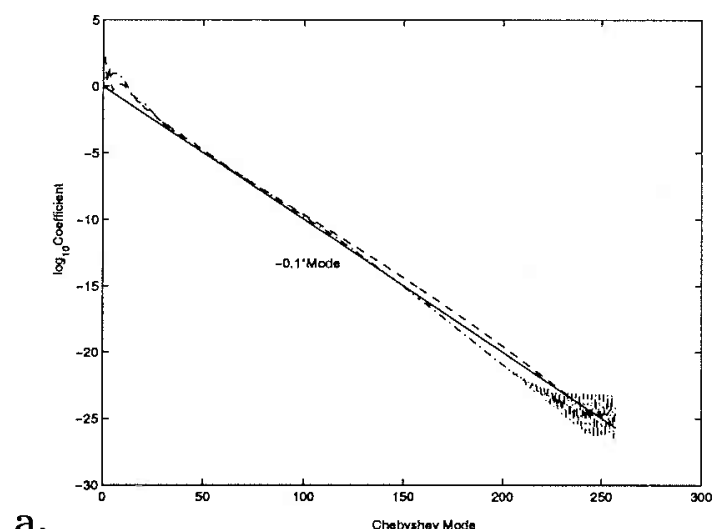

a.
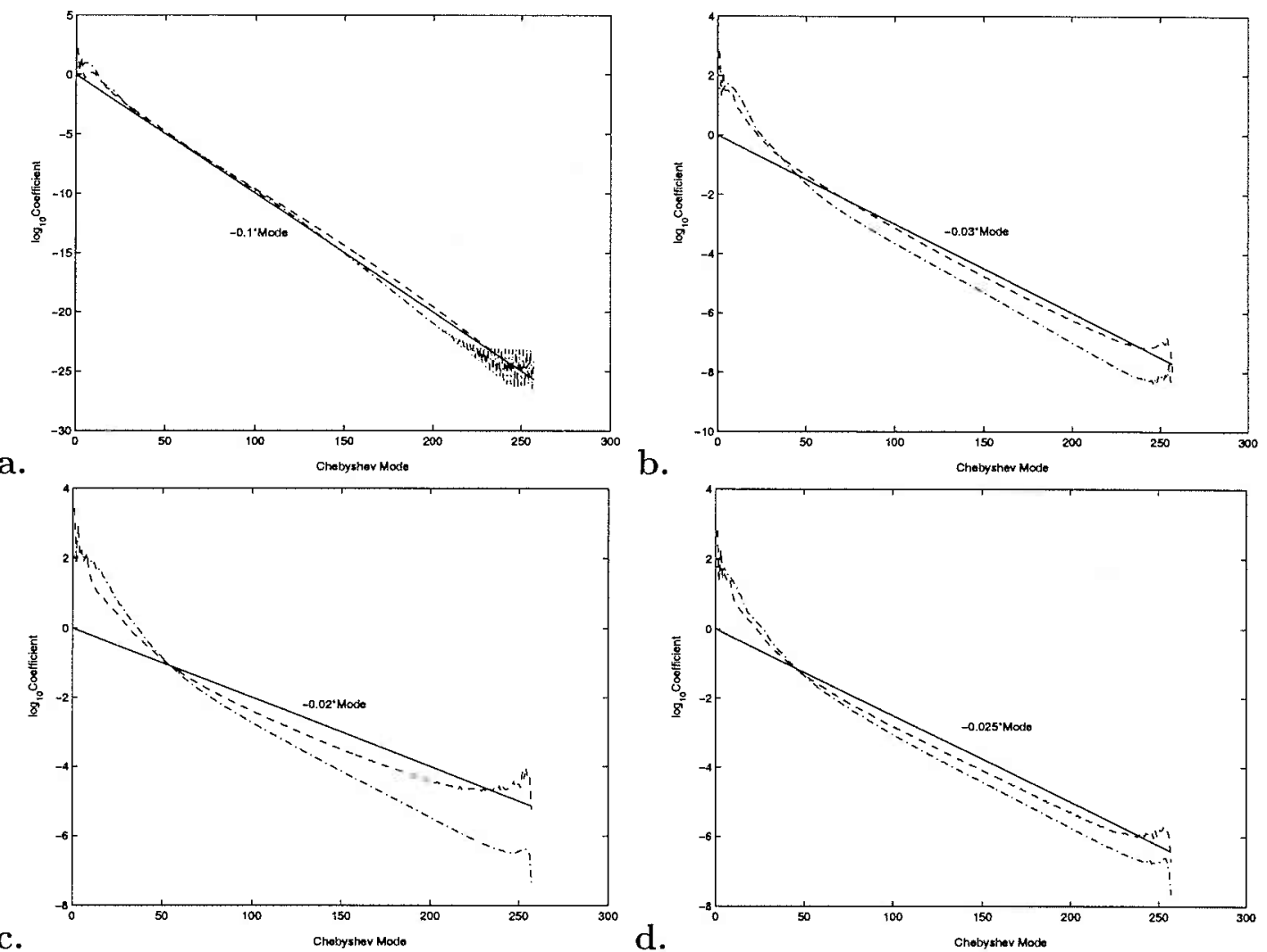

b.

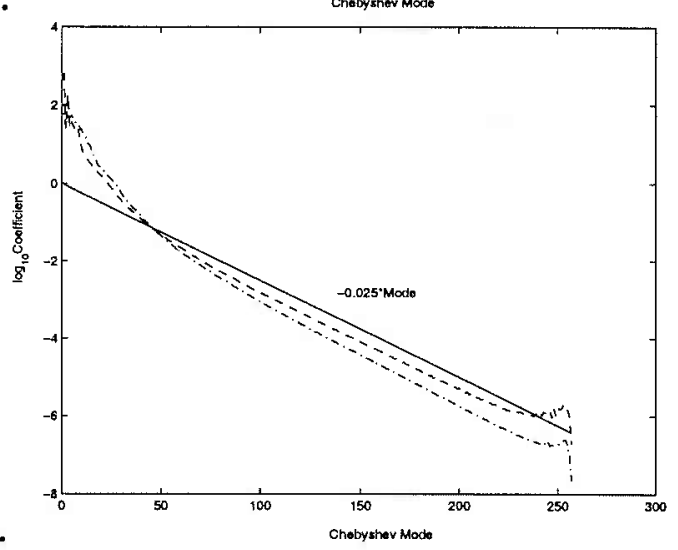

Figure C-1: Base 10 logarithm of the absolute value of Chebyshev coefficients in $y$ (averaged over $x$ modes and time) and $x$ (averaged over $y$ modes and time) versus Chebyshev polynomial mode number for the relative vorticity field of time-dependent (a) $\operatorname{Re}(\mathrm{bdy})=1, \operatorname{Re}($ int $)=1,(\mathrm{~b}) \operatorname{Re}(\mathrm{bdy})=3, \operatorname{Re}($ int $)=3,(\mathrm{c}) \operatorname{Re}(\mathrm{bdy})=5, \operatorname{Re}($ int $)=5$, (d) $\operatorname{Re}(\mathrm{bdy})=1, \operatorname{Re}(\mathrm{int})=5$ calculations. Also plotted are a straight lines at a factor times the polynomial mode number for comparison. Similar proof of spectral convergence is given in figure 3-5.

the spectral decay would continue to even smaller errors.

Finally, precision errors are evident in figure C-1a. The rapid changes in the tail of the distribution show that the errors have reached the digital limit of representation for the double precision used. The 25 orders of magnitude separation between the largest modes and the smallest ones can not be adequately represented at this precision.

Programming the Chebyshev pseudo-spectral calculation required enormous ad- 
ditional effort over using a finite-difference calculation. The difficulty in calculation per grid point is also significant. However, because the frictional terms play such an important role in these models this extra effort is warranted. Calculations of the energy dissipation of the mean flow using second-order accurate finite differences (on the same grid as used for Chebyshev collocation) rather than Chebyshev expansion revealed that the energy dissipation rate was overestimated by the finite differences by $20 \%$ or more due to numerical error. This demonstrates the difficulty in evaluation of high-order derivatives in numerical models.

The last, and likely most significant, intrinsic source of error is in the method of time stepping. The time integration is only second-order accurate, and must use a fixed time step. Due to the qualitative changes in the solutions and the irregular resolution of the Chebyshev polynomials, the Courant numbers could not be calculated in advance, but only during calculation. Thus, an initial guess of time-step was found empirically, and if required, the calculation was halted and restarted with a smaller time-step. In almost all cases, the Courant number for the implicitly calculated part of the friction was larger than one. Significant errors are likely due to the implicit time stepping scheme. However, as shown in chapters 2 and 3 , the vorticity and energetics of the mean flow work out to within a few percent. Also, some of the calculations were performed a second time with a time step reduced by a factor of five and showed no noticeable difference with reduction of time step.

There are also errors due to spatial resolution, even though the Chebyshev polynomial errors decay exponentially. Figure C-2 compares the mean streamfunctions from three calculations with different resolutions of the $\operatorname{Re}($ bdy $)=0.25, \operatorname{Re}($ int $)=7$ calculation. Although these calculations are very similar, there are still differences. Because of the high interior Reynolds number and the large gradient in viscosity, this is a worst case in resolution errors for the single-gyre. Time-stepping errors as are likely to be large as well because of the large gradient in viscosity. The multiple-gyre calculations were too expensive to perform more than once, but the errors there are 
probably comparable to those in figure C-2 based on the spectral decay of the Chebyshev coefficients. The slip calculations has much weaker gradients and therefore are significantly better resolved.

A comparision of the errors in other quantities from the calculations with $200 \times 200$, $256 \times 256$, and $300 \times 300$ resolutions is also helpful in estimating the error. The timemeans of total kinetic energy of the calculations are 68,95 , and 105 , respectively. The kinetic energies of the mean flow are 14.6, 24.2, and 25.5 respectively. Finally, the maxima of the time-mean streamfunction are $2.0,2.74$, and 2.79 , respectively. Thus, a worst case error estimate yields about a $10 \%$ error in descriptors of the mean flow. Also, the convergence of these numbers justifies the choice of $256 \times 256$ resolution for most of the no-slip calculations.

A comparison of errors from averaging different intervals is given in figure C-3. As in figure C-2, this is a particularly variable case, so it should be interpreted as a worst case scenario. The cases being compared are calculations of the $\operatorname{Re}(b d y)=0.25$, $\operatorname{Re}($ int $)=5$ case. The average is taken over times 10000 to 20000 and 20000 to 30000 . The time average kinetic energies are 49 and 69 respectively, and the kinetic energies of the mean flow are 14 and 25. Finally, the maxima of the time-mean streamfunction are 2.0 and 2.9 respectively. Thus, a worst case error estimate yields about a $40 \%$ error in descriptors of the mean flow. The majority of the differences are in the recirculation gyre, which is significantly stronger over the second interval. The vast majority of the calculations here were not as variable (see figure 3-11). Unfortunately, this error is intrinsic to the temporally chaotic calculations. The computer resources were not available to integrate these solutions for much longer times. 


\begin{tabular}{|c|c|c|c|c|c|c|c|c|c|}
\hline Location (Fig.) & $\overline{\delta_{I}}$ & $\delta_{M}$ (int) & $\delta_{M}(\mathrm{bdy})$ & $\delta_{S}$ & $L_{y}$ & $L_{x}$ & Resolution & $\Delta t$ & $\max (t)$ \\
\hline $1-3$ & 0 & 0.02 & 0.02 & 0 & 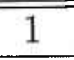 & 1 & $49 \times 49$ & 0.25 & 500 \\
\hline $2-2$ & 0.02 & 0.02 & 0.031748 & 0 & 1 & 1 & $257 \times 257$ & 0.05 & 15000 \\
\hline $2-2$ & 0.02 & 0.02 & 0.025198 & 0 & 1 & 1 & $257 \times 257$ & 0.06 & 2000 \\
\hline $1-3,2-2$ & 0.02 & 0.02 & 0.02 & 0 & 1 & 1 & $257 \times 257$ & 0.03 & 5000 \\
\hline $3-12,3-13$ & 0.02 & 0.02 & 0.02 & 0 & 1 & 1 & $257 \times 257$ & 0.025 & 10000 \\
\hline $2-2$ & 0.02 & 0.013867 & 0.031748 & 0 & 1 & 1 & $257 \times 257$ & 0.03 & 20000 \\
\hline $\begin{array}{c}2-2,2-11 \\
3-3,3-5\end{array}$ & 0.02 & 0.013867 & 0.025198 & 0 & 1 & 1 & $257 \times 257$ & 0.03 & 25000 \\
\hline $2-2$ & 0.02 & 0.013867 & 0.02 & 0 & 1 & 1 & $257 \times 257$ & 0.02 & 25000 \\
\hline $\begin{array}{c}1-3,2-2 \\
2-3,3-3,3-5 \\
3-23 \\
\end{array}$ & 0.02 & 0.013867 & 0.013867 & 0 & 1 & 1 & $257 \times 257$ & 0.025 & 10000 \\
\hline $\begin{array}{c}2-2,2-5 \\
2-9,3-1,3-3 \\
3-5,3-6,4-4 \\
4-23 \\
\end{array}$ & 0.02 & 0.011696 & 0.031748 & 0 & 1 & 1 & $257 \times 257$ & 0.01 & 20000 \\
\hline $\begin{array}{c}3-15,3-16 \\
3-27,3-29 \\
\end{array}$ & 0.02 & 0.011696 & 0.031748 & 0 & 1 & 1 & $257 \times 257$ & 0.01 & 30000 \\
\hline $2-2,2-11$ & 0.02 & 0.011696 & 0.025198 & 0 & 1 & 1 & $257 \times 257$ & 0.015 & 20000 \\
\hline $2-2$ & 0.02 & 0.011696 & 0.02 & 0 & 1 & 1 & $257 \times 257$ & 0.02 & 10000 \\
\hline $2-2$ & 0.02 & 0.011696 & 0.013867 & 0 & 1 & 1 & $257 \times 257$ & 0.015 & 10000 \\
\hline $\begin{array}{c}1-3,2-2, \\
2-6,2-9,2-13 \\
3-10,3-17,3-23 \\
4-2,4-8,4-22 \\
\end{array}$ & 0.02 & 0.011696 & 0.011696 & 0 & 1 & 1 & $257 \times 257$ & 0.02 & 10000 \\
\hline $2-2,2-12$ & 0.02 & 0.010455 & 0.031748 & 0 & 1 & 1 & $257 \times 257$ & 0.01 & 20000 \\
\hline $2-2$ & 0.02 & 0.010455 & 0.025198 & 0 & 1 & 1 & $257 \times 257$ & 0.01 & 15000 \\
\hline $2-2,2-12$ & 0.02 & 0.010455 & 0.02 & 0 & 1 & 1 & $257 \times 257$ & 0.01 & 20000 \\
\hline $2-2$ & \begin{tabular}{|l|}
0.02 \\
\end{tabular} & 0.010455 & 0.013867 & 0 & 1 & 1 & $257 \times 257$ & 0.01 & 10000 \\
\hline $2-12$ & \begin{tabular}{|l|}
0.02 \\
\end{tabular} & 0.010455 & 0.010455 & 0 & 1 & 1 & $257 \times 257$ & 0.01 & 10000 \\
\hline $2-2,2-3$ & 0.02 & 0.01 & 0.031748 & 0 & 1 & 1 & $257 \times 257$ & 0.008 & 15000 \\
\hline $\begin{array}{c}2-2,2-11 \\
3-3,3-5,3-23\end{array}$ & 0.02 & 0.01 & 0.025198 & 0 & 1 & 1 & $257 \times 257$ & 0.008 & 15000 \\
\hline $2-13$ & 0.02 & 0.009615 & 0.031748 & 0 & 1 & 1 & $257 \times 257$ & 0.008 & 15000 \\
\hline $3-21$ & $\mathrm{n} / \mathrm{a}$ & 0.04 & 0.04 & 0 & 2 & 1 & $129 \times 65$ & 0.0125 & 2500 \\
\hline $3-19,3-20$ & $\mathrm{n} / \mathrm{a}$ & 0.01 & 0.01 & 0 & 0.5 & 1 & $65 \times 129$ & 0.1 & 5000 \\
\hline $3-24,3-25$ & \begin{tabular}{|l|}
0.02 \\
\end{tabular} & 0.011696 & 0.031748 & 0.003 & 1 & 1 & $257 \times 257$ & 0.02 & 5000 \\
\hline $3-24,3-25$ & \begin{tabular}{|l|}
0.02 \\
\end{tabular} & 0.011696 & 0.011696 & 0.003 & 1 & 1 & $257 \times 257$ & 0.01 & 5000 \\
\hline $3-24,3-25$ & 0.02 & 0.011696 & 0.011696 & 0.001 & 1 & 1 & $257 \times 257$ & 0.02 & 5000 \\
\hline
\end{tabular}

Table C.1: This table presents the parameter settings for many of the time-dependent, no-slip, single-gyre calculations presented in this dissertation. 


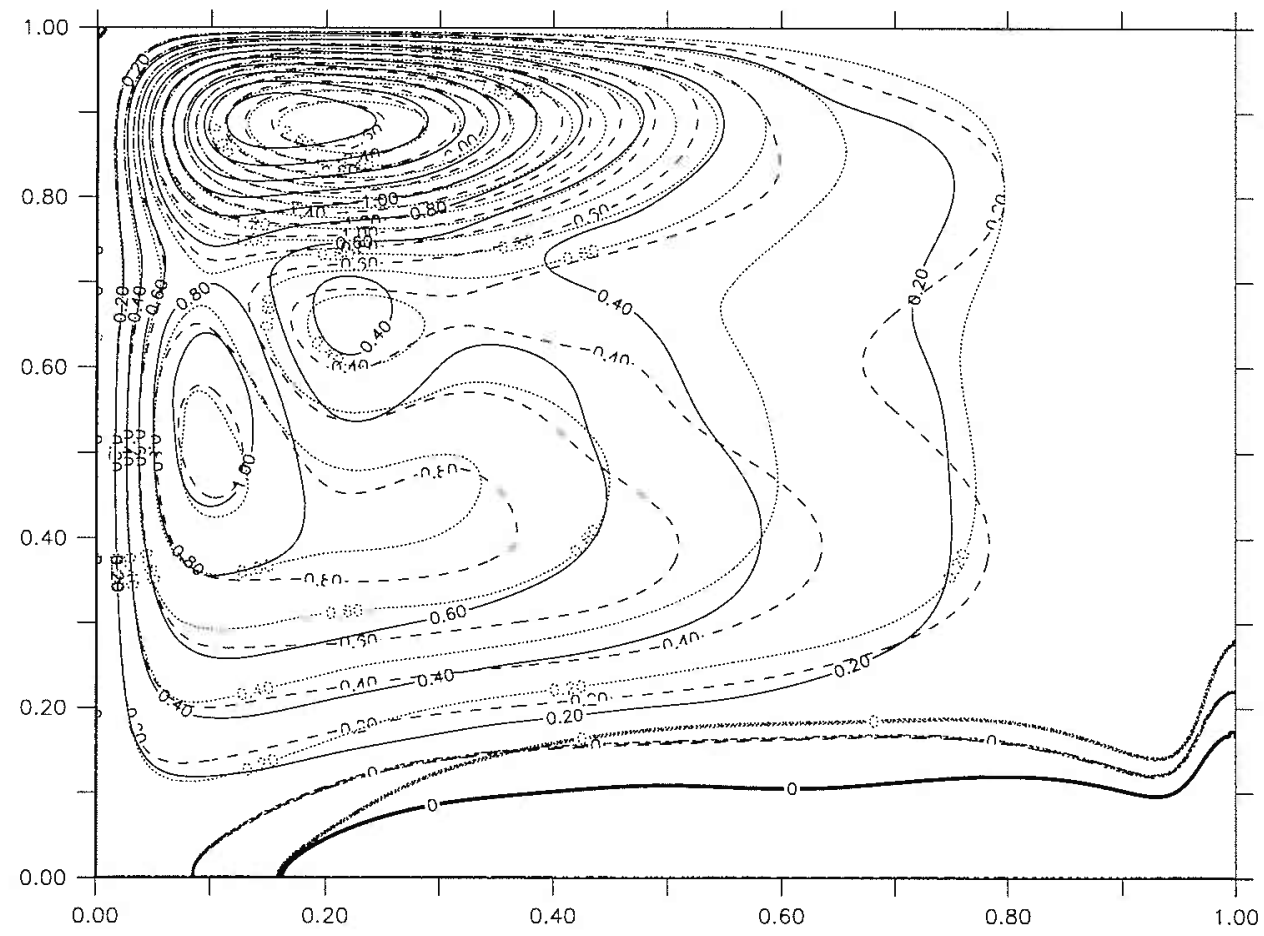

Figure C-2: Superposition of contour plots of the $\operatorname{Re}(\mathrm{bdy})=0.25, \operatorname{Re}(\mathrm{int})=7$ calculation with no-slip boundary conditions. Resolutions are 200x200 (solid), $256 \times 256$ (dashed), and $300 \times 300$ (dotted) with $\mathrm{dt}=0.008,0.01,0.015$ respectively. 


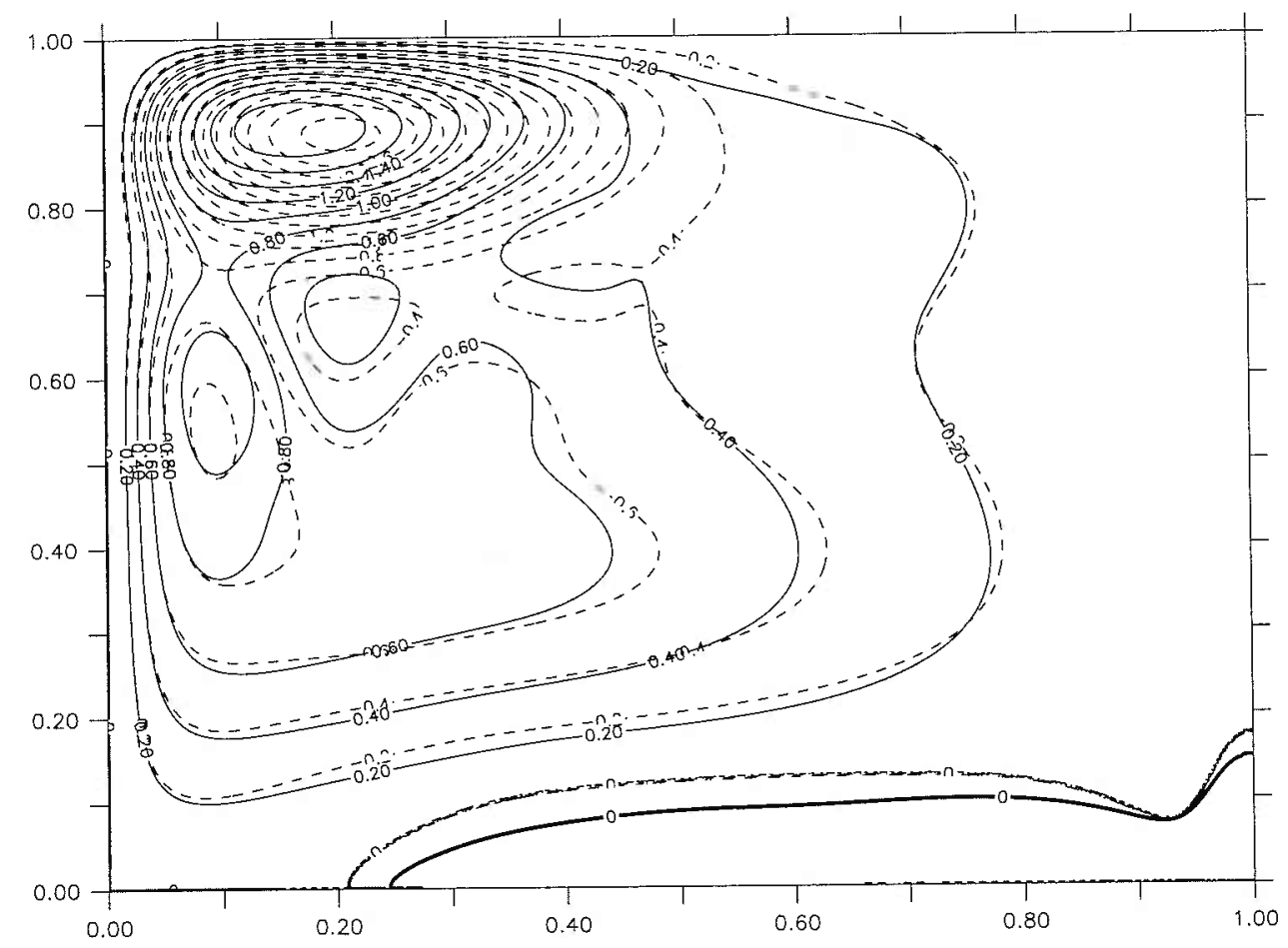

Figure C-3: Superposition of contour plots of the $\operatorname{Re}(\mathrm{bdy})=0.25, \operatorname{Re}(\mathrm{int})=5$ calculation with no-slip boundary conditions. The solid contours are the average of the streamfunction from time 10000 to 20000 and the dashed contours are the average from 20000 to 30000 . 


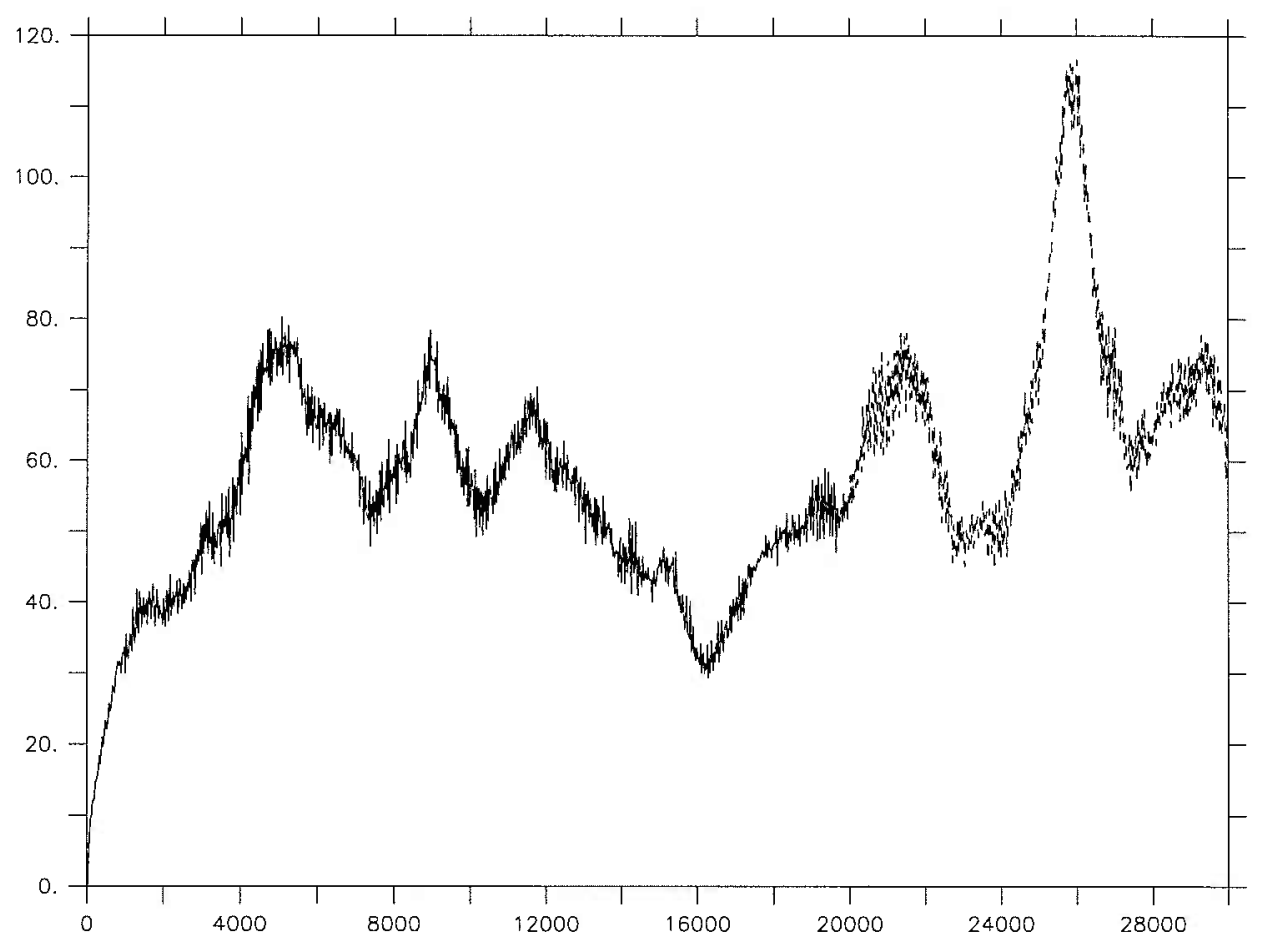

Figure C-4: Total kinetic energy of the no-slip, $\operatorname{Re}(\mathrm{bdy})=0.25, \operatorname{Re}(\mathrm{int})=5$ calculation. 


\begin{tabular}{|c|c|c|c|c|c|c|c|c|c|}
\hline \hline Location (Fig.) & $\delta_{I}$ & $\delta_{M}$ (int) & $\delta_{M}$ (bdy) & $\delta_{S}$ & $L_{y}$ & $L_{x}$ & Resolution & $\Delta t$ & $\max (t)$ \\
\hline \hline $\begin{array}{c}3-27,3-28 \\
3-29\end{array}$ & 0.02 & 0.043089 & 0.014736 & 0 & 1 & 1 & $129 \times 129$ & 0.05 & 10000 \\
\hline $\begin{array}{c}3-27,3-28 \\
3-29,4-19\end{array}$ & 0.02 & 0.014736 & 0.014736 & 0 & 1 & 1 & $65 \times 65$ & 0.0004 & 100000 \\
\hline None & 0.02 & 0.043089 & 0.014736 & 0 & 1.56 & 1 & $257 \times 129$ & 0.03 & 5000 \\
\hline $4-19,4-13$ & 0.02 & 0.014736 & 0.014736 & 0 & 1.56 & 1 & $257 \times 129$ & 0.01 & 5000 \\
\hline None & 0.02 & 0.043089 & 0.014736 & 0 & 2 & 1 & $257 \times 129$ & 0.03 & 10000 \\
\hline $4-19,4-10$ & 0.02 & 0.014736 & 0.014736 & 0 & 2 & 1 & $257 \times 129$ & 0.01 & 5000 \\
\hline $4-14$ & 0.02 & 0.011696 & 0.011696 & 0 & 1.56 & 1 & $257 \times 129$ & 0.01 & 8000 \\
\hline $4-40$ & 0.02 & 0.031748 & 0.011696 & 0 & 2 & 1 & $257 \times 129$ & 0.01 & 5000 \\
\hline $4-12$ & 0.02 & 0.031748 & 0.011696 & 0 & 2 & 1 & $301 \times 151$ & 0.005 & 5000 \\
\hline $4-15$ & 0.02 & 0.009283 & 0.009283 & 0 & 1.56 & 1 & $401 \times 257$ & 0.005 & 3620 \\
\hline
\end{tabular}

Table C.2: This table presents the parameter settings for many of the time-dependent, slip calculations presented in this dissertation.

\begin{tabular}{|c|c|c|c|c|c|c|c|c|c|}
\hline \hline Location (Fig.) & $\delta_{I}$ & $\delta_{M}$ (int) & $\delta_{M}$ (bdy) & $\delta_{S}$ & $L_{y}$ & $L_{x}$ & Resolution & $\Delta \mathrm{t}$ & $\max (t)$ \\
\hline \hline $4-4,4-23$ & 0.02 & 0.011696 & 0.031748 & 0 & 1.56 & 1 & $513 \times 257$ & 0.02 & 6000 \\
$4-2,4-7$, & 0.02 & 0.011696 & 0.011696 & 0 & 1.56 & 1 & $513 \times 257$ & 0.02 & 6000 \\
$4-22$ & & & & & & & & & \\
\hline $4-4,4-23$ & 0.02 & 0.011696 & 0.031748 & 0 & 2 & 1 & $513 \times 257$ & 0.005 & 6000 \\
$4-2,4-8$, & 0.02 & 0.011696 & 0.011696 & 0 & 2 & 1 & $513 \times 257$ & 0.005 & 6000 \\
$4-22$ & & & & & & & & & \\
\hline $4-31,4-32$ & 0.02 & 0.011696 & 0.011696 & 0 & 2 & 1 & $513 \times 257$ & 0.02 & 9500 \\
\hline $4-37$ & 0.06 & 0.0205197 & 0.0205197 & 0 & 1.56 & 1 & $301 \times 151$ & 0.004 & 5000 \\
\hline $4-37$ & 0.06 & 0.095244 & 0.0205197 & 0 & 1.56 & 1 & $257 \times 129$ & 0.003 & 5000 \\
\hline
\end{tabular}

Table C.3: This table presents the parameter settings for many of the time-dependent, no-slip, double- and two-gyre calculations presented in this dissertation. 


\section{Bibliography}

Arbic, B. K. and G. R. Flierl: 2003, Baroclinically unstable geostrophic turbulence in the limits of strong and weak ekman friction: Application to mid-ocean eddies. Journal of Physical Oceanography, submitted. 41

Balmforth, N. J. and C. Piccolo: 2001, The onset of meandering in a barotropic jet. Journal of Fluid Mechanics, 449, 85-114. 202, 216, 230

Batchelor, G. K.: 1952, Diffusion in a field of homogeneous turbulence. Journal of Fluid Mechanics, 214, 535-557. 27

- 1960, The Theory of Homogeneous Turbulence. Cambridge University Press, Cambridge, 197 pp. 27

- 1969, Computation of the energy spectrum in homogeneous two-dimensional turbulence. The Physics of Fluids, Supplement II, 233-239. 41, 42

Becker, J. M. and R. Salmon: 1997, Eddy formation on a continental slope. Journal of Marine Research, 55, 181-200. 41, 187, 257

Berkooz, G., P. Holmes, and J. L. Lumley: 1993, The proper orthogonal decomposition in the analysis of turbulent flows. Annual Review of Fluid Mechanics, 25, 539-575. 117

Berloff, P. S. and J. C. McWilliams: 2002, Material transport in oceanic gyres. part 
II: Hierarchy of stochastic models. Journal of Physical Oceanography, 32, 797-830. 201

Berloff, P. S., J. C. McWilliams, and A. Bracco: 2002, Material transport in oceanic gyres. part I: Phenomenology. Journal of Physical Oceanography, 32, 764-796. 163, 201

Böning, C. W. 1986, On the influence of frictional parameterizations in wind-driven ocean circulation models. Dynamics of Atmospheres and Oceans, 10, 63-92. 153

Bower, A. S. and T. Rossby: 1989, Evidence of cross-frontal exchange processes in the Gulf Stream based on isopycnal RAFOS float data. Journal of Marine Research, 19, 1177-1190. 164, 201

Boyd, J. P.: 1989, Chebyshev \& Fourier Spectral Methods, Springer-Verlag, Berlin. 54

Bryan, K.: 1963, A numerical investigation of a nonlinear model of a wind-driven ocean. Journal of the Atmospheric Sciences, 20, 594-606. 55

Canuto, C., M. Y. Hussaini, A. Quarteroni, and T. A. Zang: 1988, Spectral Methods in Fluid Dynamics. Springer-Verlag, Berlin. 284

Carrier, G. F and A. R. Robinson: 1962, On the theory of the wind-driven ocean circulation. Journal of Fluid Mechanics, 12, 49-80. 21

Cessi, P.: 1991, Laminar separation of colliding western boundary currents. Journal of Marine Research, 49, 697-717. 188, 189, 194

Cessi, P. and G. R. Ierley: 1990, Symmetry-breaking multiple equilibria in quasigeostrophic, wind-driven flows. Journal of Physical Oceanography, 25, 1196-1205. 232 
Cessi, P., G, R. Ierley, and W. R. Young: 1987, A model of the inertial recirculation deiven by potential vorticity anomalies. Journal of Physical Oceanography, 17, 1640-1653. 29

Cessi, P. and F. Primeau: 2001, Dissipative selection of low-frequency modes in a reduced-gravity basin. Journal of Physical Oceanography, 31, 127-137. 261

Charney, J. G.: 1955, The gulf stream as an inertial boundary layer. Proceedings of the National Academy of Sciences, 41, 731-740. 20, 51, 94, 95, 96, 246, 251

Charney, J. G. and M. E. Stern: 1962, On the stability of internal baroclinic jets in a rotating atmosphere. Journal of the Atmospheric Sciences, 19, 159-172. 146, 155

Chassignet, E. P.: 1995, Vorticity dissipation by western boundary currents in the presence of outcropping layers. Journal of Physical Oceanography, 25, 242-255. 257,258

Chassignet, E. P and R. Bleck: 1995, The influence of layer outcropping on the separation of boundary currents. part I: The wind-driven experiments. Journal of Physical Oceanography, 23, 1485-1507. 257

Chassignet, E. P., R. Bleck, and C. G. H. Rooth: 1995, The influence of layer outcropping on the separation of boundary currents. part II: The wind- and buoyancydriven experiments. Journal of Physical Oceanography, 25, 2404-2422 257

Cowley, S. J.: 2001, Laminar boundary-layer theory: A 20th century paradox? Proceedings of ICTAM 2000, H. Aref and J. W. Phillips, eds., Kluwer Academic Publishers, Dordrecht, The Netherlands, 389-411. 73

Drazin, P. G. and W. H. Reid: 1981, Hydrodynamic Stability. Cambridge University Press, Cambridge, 527 pp. 155,216 
Edwards, C. A. and J. Pedlosky: 1998, Dynamics of nonlinear cross-equatorial flow. part I: Potential vorticity transformation. Journal of Physical Oceanography, 28, 2382-2406. $35,97,158,251$

Fjørtoft, R.: 1950, Application of integral theorems in deriving criteria of stability for laminar flows and for the baroclinic circular vortex. Geofys. Publ., 17, 1-52. 146, 155

Fofonoff, N. P.: 1954, Steady flow in a frictionless homogenous ocean. Journal of Marine Research, 13, 254-262. 21, 111, 112

Fox-Kemper, B., R. Ferrari, and J. Pedlosky: 2003, On the indeterminacy of rotational and divergent eddy fluxes. Journal of Physical Oceanography, 33, 478-483. 79,173

Gottlieb, D. and S. A. Orszag: 1981, Numerical Analysis of Spectral Methods: Theory and Applications. Society for Industrial and Applied Mathematics, Philadelphia. 54,284

Greatbatch, R. J. and B. T. Nadiga: 1999, Four-gyre circulation in a barotropic model with double-gyre wind forcing. Journal of Physical Oceanography, 30, 1461-1471. 105,159

Greenspan, H. P.: 1962, A criterion for the existence of inertial boundary layers in the oceanic circulation. Proceedings of the National Academy of Sciences, 48, 2034-2039. 21, 96

Griffa, A. and S. Castellari: 1991, Nonlinear general circulation of an ocean model driven by wind with a stochastic component. Journal of Marine Research, 49, 53-73. 111

Griffa, A. and R. Salmon: 1989, Wind-driven ocean circulation and equilibrium statistical mechanics. Journal of Marine Research, 47, 457-492. 111, 112 
Haidvogel, D. B.: 1976, The sensitivity and predictability of mesoscale eddies in an idealized model ocean. Ph.D. Thesis-Massachusetts Institute of Technology. Dept. of Meteorology, 245 pp. 287, 288

Hallberg, R. and P. B. Rhines: 2000, Developments in Geophysical Turbulence, Kluwer, Dordrecht, chapter Boundary sources of potential vorticity in geophysical circulations. 51-65. 39, 187, 256

Harrison, D. E. and W. R. Holland: 1981, Regional eddy vorticity transport and the equilibrium vorticity budgets of a numerical model ocean circulation. Journal of Physical Oceanography, 11, 190-208. 37, 163, 165, 166, 171, 182, 206

Haynes, P. and M. McIntyre: 1987, On the evolution of vorticity and potential vorticity in the presence of diabatic heating and frictional or other forces. Journal of the Atmospheric Sciences, 44, 828-841, 41, 56

Haynes, P. and E. Shuckburgh: 2001, Effective diffusivity as a diagnostic of atmospheric transport 1 stratosphere. Journal of Geophysical Research, D18, 32033211. 201

Holland, W. R.: 1978, The role of mesoscale eddies in the general circulation of the ocean-numerical experiments using a wind-driven quasi-geostrophic model. Journal of Physical Oceanography, 8, 363-392. 47

Holland, W. R. and L. B. Lin: 1975, On the origin of mesoscale eddies and their contribution to the general circulation of the ocean. ii. a parameter study. Journal of Physical Oceanography, 5, 658-669. 33, 41, 105, 158

Holm, D. D. and B. T. Nadiga: submitted 2002, Modeling mesoscale turbulence in the barotropic double gyre circulation. Journal of Physical Oceanography. 105, 159 
Huang, R. X.: 1987, A 3-layer model for wind-driven circulation in a subtropicalsubpolar basin 1. model formulation and the subcritical state. Journal of Physical Oceanography, 17, 664-678. 257

Huang, R. X. and L. Chao: Submitted 2001, Water mass formation and transformations in the Pacific. Journal of Physical Oceanography. 168

Huang, R. X. and G. R. Flierl: 1987, Two-layer models for the thermocline and current structure in subtropical subpolar gyres. Journal of Physical Oceanography, 17, 872-884. 257

Hughes, C. W. and B. A. De Cuevas: 2001, Why western boundary currents in realistic oceans are inviscid: A link between form stress and bottom pressure torques. Journal of Physical Oceanography, 31, 2871-2885. 39, 246, 255, 256

Ierley, G. R.: 1987, On the onset of recirculation in barotropic general circulation models. Journal of Physical Oceanography, 17, 2366-2374. 29

- 1993, Nonlinear disturbances of western boundary currents. Journal of Physical Oceanography, 17, 2366-2374. 155

Ierley, G. R. and O. G. Ruehr: 1986, Analytic and numerical solutions of a nonlinear boundary-layer problem. Studies in Applied Math, 75, 1-36. 28, 29, 96, 97, 246, 251

Ierley, G. R. and V. A. Sheremet: 1995, Multiple solutions and advection-dominated flows in the wind-driven circulation. part I: Slip. Journal of Marine Research, 53, 703-737. 29, 38, 46, 55, 105

Ierley, G. R. and W. R. Young: 1991a, Inertial recirculation in a beta-plane corner. Journal of Physical Oceanography, 18, 683-689. 29

- 1991b, Viscous instabilities in the western boundary-layer. Journal of Physical Oceanography, 21, 1323-1332. 155 
Il'in, A. M. and V. M. Kamenkovich: 1964, The structure of the boundary layer in a two-dimensional model of ocean currents. Okeanologiya, 4, 756-769, in Russian. 97

Joliffe, I. T.: 1986, Principal Component Analysis. Springer-Verlag, New York, 271 pp. 120

Kamenkovich, V. M.: 1966, A contribution to the theory of the inerial-viscous boundary layer in a two-dimensional model of ocean currents. Isvestiya, 781-792. 97

Kamenkovich, V. M., V. A. Sheremet, A. R. Pashtushkov, and S. Belotserkovsky: 1995, Analysis of the barotropic model of the subtropical gyre in the ocean for finite reynolds numbers, part I. Journal of Marine Research, 53, 959-994. 31, 46, $55,105,158$

LaCasce, J. and A. S. Bower: 2000, Relative dispersion in the subsurface North Atlantic. Journal of Marine Research, 58, 863-894. 23, 27, 46, 55, 245

Lacasce, J. H. and J. Pedlosky: 2002, Baroclinic Rossby waves in irregular basins. Journal of Physical Oceanography, 32, 2828-2847. 261

Lamb, H.: 1932, Hydrodynamics. Dover Publications, New York, 6th edition, 737 pp. 216

Le Provost, C and J. Verron: 1987, Wind-driven ocean circulation transition to barotropic instability. Dynamics of Atmospheres and Oceans, 11, 175-201. 225, 230

Lozier, M. S. and S. C. Riser: 1989, Potential vorticity dynamics of boundary currents in a quasi-geostrophic ocean. Journal of Physical Oceanography, 19, 1373-1396. 164, 182, 201 
- 1990, Potential vorticity sources and sinks in a quasi-geostrophic ocean: beyond western boundary currents. Journal of Physical Oceanography, 20, 1608-1627. 37, $163,164,166,182,200,201,205,206,249$

Luyten, J. R., J. Pedlosky, and H. M. Stommel: 1983, The ventilated thermocline. Journal of Physical Oceanography, 13, 292-309. 14

Maps, E. T.: 1988, Data announcement 88-mgg-02, digital relief of the surface of the Earth. Technical report, NOAA, National Geophysical Data Center, Boulder, Colorado. 256

Marshall, D. P.: 2001, An implicit formula for boundary current separation. Journal of Physical Oceanography, 31, 1633-1638. 212

Marshall, J. C.: 1984, Eddy mean flow interaction in a barotropic model. Quarterly Journal of the Royal Meteorological Society, 100, 573-590. 37, 44, 163, 165, 166, $167,171,172,182,206$

Marshall, J. C., H. Jones, R. Karsten, and R. Wardle: 2002, Can eddies set ocean stratification? Journal of Physical Oceanography, 32, 26-38. 262

Marshall, J. C. and G. Nurser: 1992, Fluid dynamics of oceanic thermocline ventilation. Journal of Physical Oceanography, 22, 1315-1329. 56

Marshall, J. C. and G. Shutts: 1981, A note on rotational and divergent eddy fluxes. Journal of Physical Oceanography, 11, 1677-1680. 47

McWilliams, J. C., M. J. Molemaker, and I. Yavneh: 2001, From stirring to mixing of momentum: Cascades from balanced flows to dissipation in the oceanic interior. 'Aha Huliko'a Proceedings, P. Miller and D. Henderson, eds., SOEST Special Publication, Manoa, Hawaii, 59-66. 259

McWilliams, J. C., J. B. Weiss, and I. Yavneh: 1994, Anisotropy and coherent structures in planetary turbulence. Science, 264, 410-413. 31 
Morgan, G. W.: 1956, On the wind-driven ocean circulation. Tellus, 8, 301-320. 21

Munk, W. H.: 1950, On the wind-driven ocean circulation. Journal of Meteorology, 7, 79-93. 16, 17, 22, 51, 94, 145

Munk, W. H. and G. F. Carrier: 1950, The wind-driven circulation in ocean basins of various shapes. Tellus, 2, 158-167. 16, 146

Orszag, S. A. and M. Israeli: 1974, Numerical simulations of viscous incompressible Hows. Annual Review of Fluid Mechanics, 6, 281-318. 38, 284

Özgökmen, T. and E. P. Chassignet: 1998, Emergence of inertial gyres in a two-layer quasigeostrophic ocean model. Journal of Physical Oceanography, 28, 461-484. 111

Parsons, A. T.: 1969, A two-layer model of gulf stream separation. Journal of Fluid Mechanics, 39, 511--528. 257, 258

Pedlosky, J.: 1965a, A note on the western intensification of the oceanic circulation. Journal of Marine Research, 23, 207-209. 18, 22, 97

-- 1965b, A study of the time dependent ocean circulation. Journal of the Atmospheric Sciences, 22, 267-272. 106, 108, 109, 110, 112, 116, 137, 159, 252

- 1987, Geophysical Fluid Dynamics. Springer, Berlin, second edition, 710 pp. 55, 115

- 1996, Ocean Circulation Theory. Springer, Berlin, 453 pp. 53

Pierrehumbert, R. T.: 1991, Large-scale horizontal mixing in planetary atmospheres. Phys. Fluids, 1250-1260. 201

Plumb, R. A.: 1982, A new look at the energy cycle. Journal of the Atmospheric Sciences, 40, 1669-1688. 141 
Pratt, L. J., M. S. Lozier, and N. Beliakova: 1995, Parcel trajectories in quasigeostrophic jets: Neutral modes. Journal of Physical Oceanography, 25, 1451-1466. 163,202

Press, W. H., S. A. Teukolsky, W. T. Vetterling, and B. P. Flannery: 1992, Numerical Recipes in C. Cambridge University Press, Cambridge, United Kingdom, 2 edition, 993 pp. 278

Primeau, F.: 2002, Long Rossby wave basin-crossing time and the resonance of lowfrequency basin modes. Journal of Physical Oceanography, 32, 2652-2665. 261

Radko, T. and J. C. Marshall: 2002, Eddy-induced diapycnal fluxes and their role in the maintenance of the thermocline, submitted. 262

Rhines, P. B.: 1975, Waves and turbulence on a beta plane. Journal of Fluid Mechanics, 69, 417-443. 41,42

Rhines, P. B. and W. R. Young: 1984, Homogenization of potential vorticity in planetary gyres. Journal of Fluid Mechanics, 122, 347-367. 72

Roberts, M. J. and D. P. Marshall: 2000, On the validity of downgradient eddy closures in ocean models. Journal of Geophysical Research, 105, 28,613-28,627. 172

Rogerson, A. M., P. D. Miller, L. J. Pratt, and C. K. R. T. Jones: 1999, Lagrangian motion and fluid exchange in a barotropic meandering jet. Journal of Physical Oceanography, 29, 2635-2655. 163, 202, 203, 230

Salmon, R., G. Holloway, and M. C. Hendershott: 1976, The equilibrium statistical mechanics of simple quasi-geostrophic models. Journal of Fluid Mechanics, 75, 691-703. 111 
Scott, R. B. and D. N. Straub: 1998, Small Viscosity Behavior of a Homogeneous, Quasigeostrophic, Ocean Circulation Model. Journal of Marine Research, 56, 12251258. 111,142

Sheremet, V. A.: 2002, Inertial gyre driven by a zonal jet emerging from the western boundary. Journal of Physical Oceanography, 32, 2361-2378. 30

Sheremet, V. A., G. R. Ierley, and V. M. Kamenkovich: 1997, Eigenanalysis of the two-dimensional wind-driven ocean circulation problem. Journal of Marine Research, 55, 57--92. 38, 46, 115, 117, 123, 128, 153

Sheremet, V. A., V. M. Kamenkovich, and A. R. Pashtushkov: 1995, Analysis for the barotropic model of the subtropical gyre in the ocean for finite reynolds numbers, part II. Journal of Marine Research, 53, 995-1024. 46, 105, 158

Smith, K. S., G. Boccaletti, C. C. Henning, I. Marinov, C. Y. Tam, I. M. Held, and G. K. Vallis: 2002, Turbulent diffusion in the geostrophic inverse cascade. Journal of Fluid Mechanics, 469, 13-48. 41

Smith, K. S. and G. K. Vallis: 2001, The scales and equilibration of midocean eddies: freely evolving flow. Journal of Physical Oceanography, 31, 554-571. 41

- 2002, The scales and equilibration of midocean eddies: forced-dissipative flow. Journal of Physical Oceanography, 32, 1699-1720. 41

Stommel, H. M.: 1948, The westward intensification of wind-driven ocean currents. Transactions, American Geophysical Union, 29, 202-206. 11, 15, 17, 22, 33, 51, 94, 145

Sverdrup, H. U.: 1947, Wind-driven currents in a baroclinic ocean; with application to the equatorial currents of the eastern Pacific. Proceedings of the National Academy of Sciences, 33, 318-326. 13, 17, 19 
Taylor, G. I.: 1921, Diffusion by continuous movements. Proceedings of the London Mathematical Society, 20, 196-212. 25

Tennekes, H. and J. L. Lumley: 1972, A First Course in Turbulence. MIT Press, Cambridge, 300 pp. 27

Veronis, G.: 1966a, Wind-driven ocean circulation-part I. linear theory and perturbation analysis. Deep-Sea Research, 13, 17-29. 146

- 1966b, Wind-driven ocean circulation-part II. numerical solution of the nonlinear problem. Deep-Sea Research, 13, 30-55. 29, 45, 146

- 1973, Model of world ocean circulation. part I: Wind-driven, two-layer. Journal of Marine Research, 31, 228-288. 257

Verron, J. and C. Le Provost: 1991, Response of eddy-resolved general circulation numerical models to asymmetrical wind forcing. Dynamics of Atmospheres and Oceans, 15, 505-533. 192

Webster, F.: 1961, The Effect of Meanders on the Kinetic Energy Balance of the Gulf Stream. Tellus, 13, 392-401. 139, 141

Welander, P.: 1964, Note on the role of boundary friction in the wind-driven ocean circulation. Tellus, 16, 408-410. 16

Wunsch, C.: 1996, The Ocean Circulation Inverse Problem. Cambridge University Press, Cambridge; New York. 117

- 1997, The vertical partition of oceanic horizontal kinetic energy. Journal of Physical Oceanography, 27, 1770-1794. 120

- 2002, Meteorology at the Millenium, Academic Press, San Diego, chapter Ocean Observations and the Climate Forecast Problem. 233-245. 245 